\title{
Mining into interspecific bacterial interactions
}

Olaf Tyc 


\section{Thesis committee}

\section{Promotor}

Prof. Dr W. de Boer

Professor of Microbial Soil Ecology

Wageningen University

\section{Co-promotor}

Dr P. Garbeva

Senior researcher

Netherlands Institute of Ecology (NIOO-KNAW), Wageningen

\section{Other members}

Prof. Dr H. Smidt, Wageningen University

Dr A.L.C. Jousset, Utrecht University

Dr M. Ongena, University of Liège, Belgium

Prof. Dr G.P. van Wezel, Leiden University

This $\mathrm{PhD}$ research was conducted under the auspices of the C.T. de Wit Graduate School for Production Ecology \& Resource Conservation (PE\&RC) 


\section{Mining into interspecific bacterial interactions}

Olaf Tyc

\section{Thesis}

submitted in fulfillment of the requirements for the degree of doctor at Wageningen University by the authority of the Rector Magnificus Prof. Dr A. P. J. Mol, in the presence of the

Thesis committee appointed by the Academic Board to be defended in public

on Friday $23^{\text {rd }}$ of September 2016 at 11 a.m. in the Aula. 
Olaf Tyc

Mining into interspecific bacterial interactions

234 pages.

$\mathrm{PhD}$ thesis, Wageningen University, Wageningen, NL (2016)

With references, with summary in English

ISBN: 978-94-6257-834-0

DOI: $10.18174 / 383745$ 
"The role of the infinitely small in nature is infinitely great."

-Louis Pasteur- 



\section{Table of Contents}

Abstract 9

Chapter 1 Introduction 11

Chapter 2 Impact of interspecific interactions on 21 antimicrobial activity among soil bacteria

Chapter 3 Volatiles in interspecific bacterial interactions $\quad \mathbf{6 1}$

Chapter 4 Exploring bacterial interspecific interactions 95 for the discovery of novel antimicrobial compounds

Chapter 5 The effect of phylogenetically different bacteria 145 on the fitness of Pseudomonas fluorescens in sand microcosms

Chapter 6 No apparent costs for facultative antibiotic 161 production by the soil bacterium Pseudomonas fluorescens Pfo-1

Chapter 7 General discussion

References 191

Summary 217

Samenvatting 221

Acknowledgments 225

PE \& RC Training \& Education Statement 229

List of publications 231

Curriculum vitae 


\section{Abstract}

In terrestrial ecosystems bacteria live in complex multi-species networks. Within those networks bacteria are constantly interacting with each other and secondary metabolites like antibiotics have an important role in these interactions. Several studies revealed that the production of secondary metabolites by soil bacteria can be influenced by the presence of other microorganisms in their vicinity. Yet, not much is known on the frequency of interaction-mediated shifts of antibiotic production in microbes.

My thesis research focused on the importance of interaction-mediated shifts in secondary metabolite production in soil bacteria. To obtain more insight in the frequency of such events a high-through-put screening method was developed and applied. In total 146 bacteria were screened for the production of antimicrobials during one-to-one confrontations and during 2798 random interactions. Antimicrobial activity was recorded via an agar-overlay assay using two target organisms: Escherichia coli WA321 and Staphylococcus aureus $533 \mathrm{R} 4$. From all tested isolates, $33 \%$ showed antimicrobial activity only in monoculture and $42 \%$ of all isolates showed activity only during interactions. More bacterial isolates showed antimicrobial activity against $S$. aureus than against $E$. coli. The frequency of interaction-mediated induction of antimicrobial activity was $6 \%$ (154 interactions out of 2798) indicating that only a specific set of species interactions induced antimicrobial activity. The screening revealed also interaction-mediated suppression of antimicrobial activity for $22 \%$ of all tested combinations.

In another line of research, it was examined how interspecific bacterial interactions affect the composition and antimicrobial activity of volatile metabolites. The identities and antimicrobial activities of the volatiles were determined in monoculture as well as in mixed cultures of four bacterial strains belonging to different genera: Chryseobacterium, Dyella, Janthinobacterium and Tsukamurella. Antimicrobial activity of the produced volatiles was assessed against fungal, oomycetal and bacterial model organisms. The results revealed that interspecific bacterial interactions affected the composition of volatiles. Fungi and oomycetes showed high sensitivity towards bacterial volatiles whereas the effect of volatiles on bacteria varied. In total 35 volatile compounds were detected most of which were sulfur-containing compounds. 
Two bacteria, namely a Gram-negative Burkholderia and a Gram-positive Paenibacillus strain, were selected to examine how interspecific bacterial interactions affect fitness, gene expression and the production of secondary metabolites. The bacteria were grown in monoculture and in mixed culture and subjected to detailed metabolome, volatolome and transcriptome analysis. One distinct volatile (2,5-bis(1-methylethyl)-pyrazine)-and one nonvolatile compound (a pederin like compound) were detected in higher concentrations during interspecific interaction. The interacting bacteria as well as the identified volatile compound were tested in bioassays and showed strong inhibitory activity against a range of plant and human pathogens such as Rhizoctonia solani, Fusarium culmorum, Candida albicans, S.aureus and E.coli.

Furthermore, it was examined how nutrient conditions influence interactions between Pseudomonas fluorescens strain Pfo-1 and two other bacteria: Pedobacter sp. $\mathrm{V}_{4} 8$ and Bacillus sp. V102. Results of incubations in sandmicrocosms revealed that under both nutrient conditions confrontation with the Gram-positive Bacillus led to significant lower cell numbers of Pseudomonas, whereas confrontation with the Gram-negative Pedobacter did not affected the growth of Pseudomonas. However, when Pseudomonas was confronted with the mixture of both strains, no significant effect on the growth of Pseudomonas was observed. Quantitative real-time PCR analysis revealed up-regulation of genes involved in the production of a broadspectrum antibiotic by Pseudomonas when confronted with Pedobacter but not when confronted with Bacillus. Finally, possible costs of antibiotic production for Pseudomonas fluorescens Pfo-1 were measured by monitoring changes in growth rate with and without induction of antibiotic production. Our results did not reveal any significant costs for the production of the antibiotics produced by Pseudomonas fluorescens Pfo-1.

In summary, this thesis extends the knowledge about the effect of interspecific bacterial interactions on secondary metabolites production (soluble and volatiles), gene expression and fitness in bacteria. The exploitation of such bacterial interspecific interactions can be an important "tool" for the discovery of novel antimicrobial and agro-chemical compounds. Additionally, the obtained knowledge can help in selecting the right players for synthetic communities that fulfil important ecosystem services, like disease suppression, in agricultural crop systems. 
Chapter 1

\section{Introduction}




\section{Microbial soil ecology}

Bacteria are the most ubiquitous and diverse living organisms on earth and surround us everywhere, with the highest diversity reported for soils (Curtis et al., 2002; Torsvik and Ovreas, 2002). Based on 16S rRNA bacterial gene sequences analysis $1 \mathrm{~g}$ of soil can contain up to 50.000 bacterial species and more than $10^{8}$ bacterial cells (Torsvik and Ovreas, 2002;Huse et al., 2008;Uroz et al., 2010).

Soil is a very complex, heterogeneous and nutrient limited environment consisting of an inorganic-organic solid matrix, in which liquid and gaseous filled pores are present. Here micro- and macro-organisms are constantly interacting and competing for nutrients and micro-habitats. Microbial communities play a key role in many ecosystem processes e.g. decomposition, mineralization, carbon sequestration and plant growth promotion (Fitter et al., 2005; Hayat et al., 2010;Richardson and Simpson, 2011). Within the huge microbial diversity there is functional redundancy: many soil bacterial species have overlapping ecological niches as they are able to use similar substrates as an energy source for their growth (Yin, 2000;Demoling et al., 2007;Strickland, 2009). Since nutrient availability is one of the limiting factors for the growth of soil bacteria, interspecific competition for nutrients and space is ongoing and is consequently one of the most abundant forms of interaction occurring in soil (Demoling et al., 2007;Rousk and Baath, 2007). Besides biotic factors like competition also abiotic factors like soil moisture content, soil $\mathrm{pH}$, matrix potential and other physio- chemical characteristics influence the survival of microorganisms. The dispersal of microorganisms in soil is strongly influenced by the moisture content, a low soil moisture content leads to lower connectivity between soil pores and thus to a lower number of accessible micro-habitats. The abiotic factors vary between soil habitats and can change locally very rapidly, thus creating a huge variety of micro-niches for growth and persistence of microbial species.

\section{The rhizosphere}

The plant root surfaces and the surrounding rhizosphere are a "hot-spot" of microbial life by providing significant sources of organic nutrients in nutrient poor surroundings. Easily degradable carbon compounds released by plant roots (root exudates) provide microorganisms with energy resources, thus microbial life intensifies in this narrow zone around the plant roots (Bais et al., 2006;De Boer et al., 2006;Dennis et al., 2010). In the rhizosphere many microbe-microbe as well as plant-microbe interactions are operating which 
have a significant contribution to the performance of plants, for instance in acquisition of inorganic nutrients (Van Der Heijden et al., 2008;Bonkowski et al., 2009;Buée et al., 2009; Lambers et al., 2009). Additional to interactions with bacteria, plants also establish beneficial relationships with other microorganisms such as mycorrhizal fungi that colonize the inside of plant roots to exchange nutrients with their host (Bonfante and Anca, 2009). The lifestyle of bacteria can range from free living in soil and rhizosphere to endoand epiphytic, including obligate endosymbionts and plant pathogens (Glick, 1995;Compant et al., 2005;Hardoim et al., 2008;Reinhold-Hurek and Hurek, 2011). Most of the interactions in the rhizosphere are beneficial for plants, however next to beneficial microorganisms the rhizosphere harbors also harmful microorganisms like pathogenic bacteria, fungi and plant-parasitic nematodes that can exert negative effects on plants and reducing crop yields worldwide (Oerke, 2006; Raaijmakers et al., 2009).

\section{Competitive strategies of soil microbes}

To sustain under such demanding environmental conditions soil bacteria evolved different survival strategies. An important strategy to enhance the competitive abilities is the production of secondary metabolites with antimicrobial properties (e.g. antibiotics, siderophores, bacteriocins, volatiles and others) called interference competition. (Velicer, 2003;Hibbing et al., 2010). The competitive interactions of microorganisms for nutrients and space are one of the fundamental mechanisms determining the composition and diversity of soil microbial communities. Interference competition involves direct interactions between the competitors via production of antimicrobial metabolites that can be targeted against closely related strains e.g. bacteriocins or against a wide range of competitors e.g. broad-spectrum antibiotics (Riley and Wertz, 2002a;b;Riley et al., 2003).

The production of secondary metabolites with antimicrobial properties is one of the strongest bacterial weapons used in the competition for space and nutrients. Next to interference competition also exploitation competition occurs in natural soil microbial communities. This type of competition involves the fast utilization of available resources without direct interactions between the competitors (Crespi, 2001;Velicer, 2003;Fiegna and Velicer, 2005;Hibbing et al., 2010). Thus, the ability to cope with the presence of competing microorganisms is essential for the growth and survival of bacteria in soil ecosystems. 


\section{Impact of interspecific bacterial interactions on secondary metabolite production}

Several studies have demonstrated that bacteria can be triggered to produce antimicrobial compounds during interspecific interactions with other bacteria (de Boer et al., 2007;Garbeva et al., 2011b;Seyedsayamdost et al., 2012;Bertrand et al., 2014). Apparently the majority of soil bacteria do not constantly produce secondary metabolites under standard laboratory conditions, as these conditions are very artificial compared to the complex situation bacteria experience in nature. So far, most of the antibiotics were identified from cultivable soil bacteria and in particular from bacteria of the genus Streptomyces spp. (Handelsman, 2005;Baltz, 2008). Indeed, next generation sequencing data of bacterial genomes revealed that many bacteria possess gene clusters encoding for so far unidentified secondary metabolites (Scherlach and Hertweck, 2009). Thus, it is possible that the actual screening procedures to explore antimicrobial compounds do overlook bacteria that possess cryptic genes encoding for novel antimicrobial compounds, due to the fact that they are only produced during interspecific interactions that are not presented in standard laboratory cultivation (Bertrand et al., 2014; Marmann et al., 2014). Triggering of antibiotic production via interactions would be one option to activate such cryptic genes.

\section{The ecological roles of secondary metabolites with antimicrobial activity}

In the soil antimicrobial compounds usually occur at concentrations below the inhibitory concentration. Therefore, there is a debate on the actual ecological role in nature which is to date not entirely known. Several studies showed that antimicrobial compounds have a variety of other roles, for example they can act as signalling molecules at sub-inhibitory concentrations and affect many cellular functions such as cellular development (Straight et al., 2006), biofilm formation, motility, virulence and nutrient use (Hoffman et al., 2005; Linares et al., 2006; Romero et al., 2011;Vaz Jauri et al., 2013). Additionally sub-inhibitory concentrations of antibiotics have shown to effect the global transcriptional pattern in bacteria by up-or- down-regulation of genes in bacteria (Goh et al., 2002;Yim et al., 2011). So far, there are only a limited number of studies demonstrating that antibiotic compounds are produced in situ by soil bacteria and at concentrations that reach their supposed functions as weapons in microbial warfare (Monier et al., 2011;Raaijmakers and Mazzola, 2012). However, sub-inhibitory concentrations of antibiotics might be still functional in interspecific competition for 
microhabitats and act as repellent against competitors. Although antimicrobial compounds can inhibit or kill other strains competing for the same ecological niche, in rare cases it has been shown that antibiotics can act also as a source of nutrients promoting the growth of bacteria under nutrient deprived conditions (D'Costa et al., 2006;Dantas et al., 2008). However, contrary to the findings by Dantas a study done by Walsh and co-workers was not able to confirm the previous findings, thus it is still questionable if antibiotics can act as a source of nutrients (Walsh et al., 2013). Hence, there is so far no scientific consensus on the ecological role of antimicrobial compounds in nature (Figure 1.1). Bacterial secondary metabolites with antimicrobial properties can be useful tools in the so called microbial warfare e.g. to protect the eukaryotic host against diseases (Raaijmakers and Mazzola, 2012;Sengupta et al., 2013). Indeed, numerous studies have demonstrated that secondary metabolites like e.g. antibiotics, enzymes and others produced by plant-associated bacteria are important for the suppression of plant pathogens and useful for biocontrol against soil-borne plant diseases (Haas and Defago, 2005; Mendes et al., 2013;Berg et al., 2014).

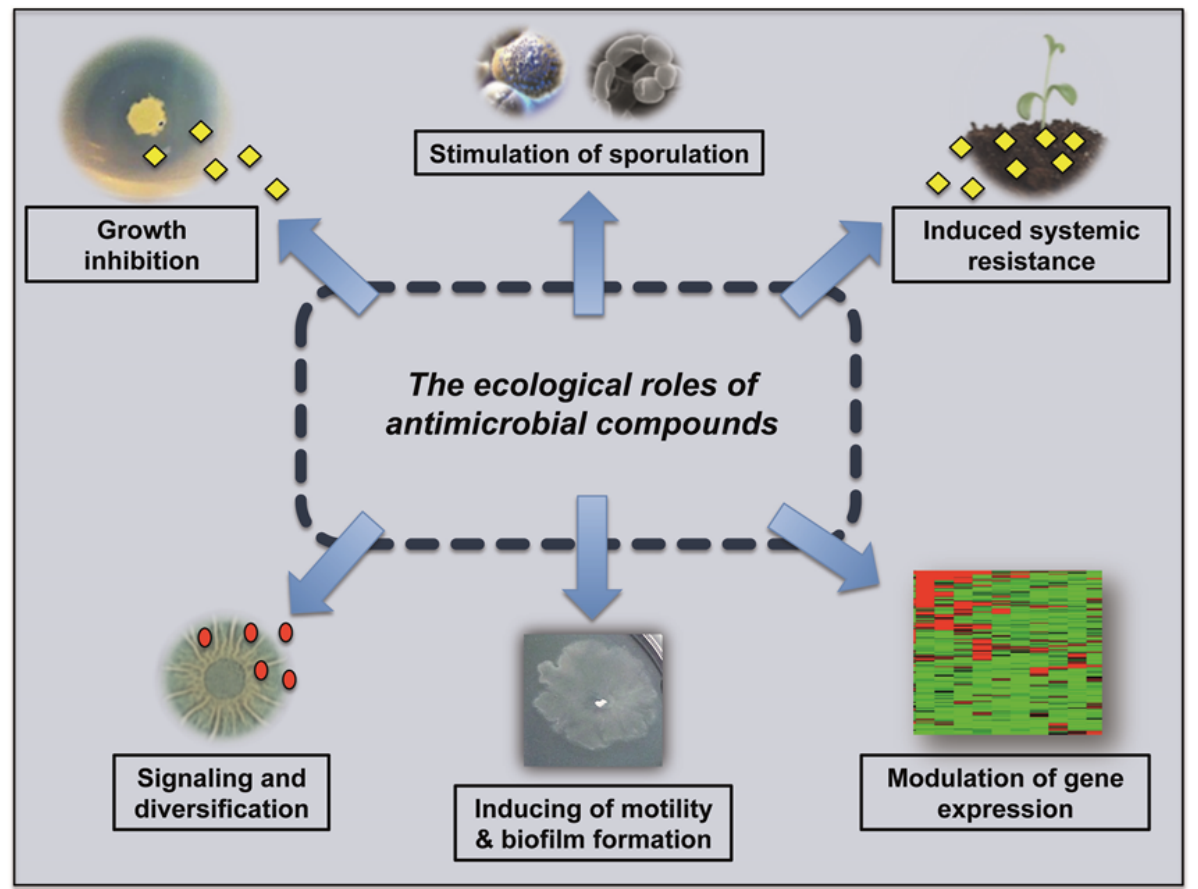

Figure 1.1: Schematic representation of the possible ecological roles of antimicrobial compounds in nature. 


\section{Volatile organic compounds (VOCs)}

Next to soluble secondary metabolites bacteria produce also a vast repository of gaseous secondary metabolites, known as volatile organic compounds (VOCs). Volatile organic compounds are small molecules characterized by a low molecular weight ( $<300 \mathrm{Da}$ ), which evaporate and diffuse easily through air- and water-filled soil pores (Schulz and Dickschat, 2007;Penuelas et al., 2014). These physiochemical properties make VOCs ideal molecules for longdistance communication and antagonistic interactions between soil microorganisms. Volatile organic compounds emitted by soil microorganisms can have various functions such as info-chemicals, growth stimulants, growth inhibitors and inhibitors of quorum sensing (Kai et al., 2009; Chernin et al., 2011;Effmert et al., 2012;Kim et al., 2013). Moreover VOCs emitted by soil microorganism have shown to promote plant growth, induced systemic resistance (ISR) and induced systemic tolerance (IST) (Ryu et al., 2003; Ryu et al., 2004). The emitted volatile blend composition may vary according to the growth conditions, in particular to the growth media composition (Cleason, 2006; Groenhagen et al., 2013;Garbeva et al., 2014a), pH, moisture content, oxygen availability and cultivation temperature (Bjurman, 2007;Insam and Seewald, 2010;Romoli et al., 2014). The investigation of volatiles emitted by soil bacteria attracts great scientific interest since their ecological role in nature is not yet fully understood. However, volatile organic compounds emitted by bacteria may play an important role in the natural buffering against soil-borne plant- diseases (Garbeva et al., 2011a;van Agtmaal et al., 2015).

\section{The need for novel antibiotics}

Facing the worldwide problem of increasing antibiotic resistance in pathogenic bacteria (Figure 1.2A) novel antimicrobial compounds are urgently needed (Crisóstomo et al., 2001;Tenover et al., 2001;Al-Gheethi et al., 2013;Economou et al., 2013). Antibiotic resistance has become one of the major sanitary problems worldwide as many bacteria have developed antibiotic resistance against the most commonly used chemical classes of antibiotics. Unfortunately, only two families of new antibiotics (lipopeptides \& oxazolidinones) have reached the clinical practice in the last four decades (Figure 1.2B) (Barbachyn and Ford, 2003;Kern, 2006;ECDC/EMEA, 2009) despite the fact the sanitary costs related to infections with antibiotic resistant microorganisms is continuously growing. The European Medicine Agency (EMA) estimates the costs that these infections provoke at approximately 1,5 billion euros only in the European Union (ECDC/EMEA, 2009). However, 
there are still possibilities to change this current situation. One major strategy to combat this rising problem is the search for new antimicrobial compounds from natural sources like soil bacteria and other organisms by implementing innovative screening techniques. So far, most of the screenings for the discovery of novel bioactive compounds do often only target well-examined genera like e.g. Streptomyces spp. that produce antibiotics in monocultures in liquid and/or in solid media. These screening methods do not consider the ecological context of antibiotic production such as the importance of interspecific interactions. Thus, new strategies are needed to access the full genetic potential of terrestrial microbes as many bacterial species that might be considered to be of no interest for exploitation based upon individual screening methods may in fact possess cryptic genes encoding for novel antimicrobial compounds that are only produced during interspecific interactions or other natural conditions.
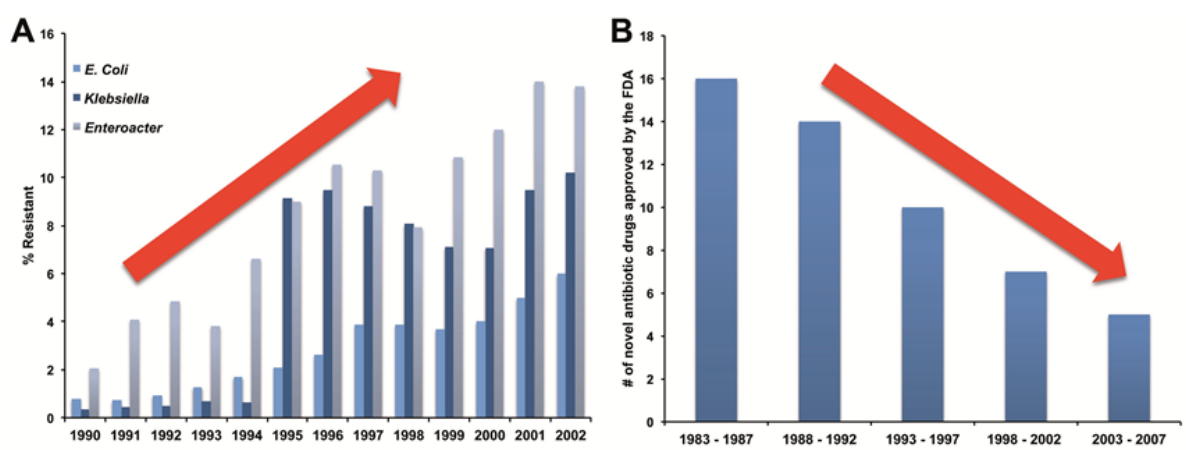

Figure 1.2: Increased drug resistance among diverse human pathogens versus the reduced development of novel antimicrobial drugs. (A) Growing proportion of human pathogens resistant against the antimicrobial ciprofloxacin. (B) The number of novel antimicrobial drugs approved by the FDA between 1983 - 2007. Data obtained and modified from (Livermore, 2004) (A) and (Spellberg et al., 2008)(B). 


\section{Objectives, research questions and thesis outline}

The major goal of this PhD research was to explore the effect of interspecific bacterial interactions on the production of secondary metabolites (soluble and volatile) with inhibitory capacities for a range of soil bacteria. We started with the knowledge on a limited number of interactions between Pseudomonas fluorescens Pfo-1 and three other soil bacteria namely Bacillus sp V102, Brevundimonas sp. V52 and Pedobacter sp. $\mathrm{V}_{48}$. In previous research done by de Boer and Garbeva it was shown that interactions between Pseudomonas fluorescens Pfo-1, Pedobacter sp. V48 and Bacillus sp. V102 had profound effects on the secondary metabolite production, fitness and gene expression in Pseudomonas fluorescens Pfo-1. The interspecific interaction between the bacteria induced the production of an antimicrobial compound in Pseudomonas fluorescens Pfo-1 that was not produced in monocultures of Pseudomonas fluorescens Pfo-1 (Garbeva et al. 2010, 2011, Garbeva \& de Boer 2009, de Boer et al., 2007). These studies revealed that apparently non-antagonistic bacteria can be triggered to produce antimicrobial compounds.

Part of this thesis dealt with the assessment of the frequency of competitorinduced antimicrobial compound production among soil bacteria, since the frequency of this event was not known so far. For this purpose I developed a high-through-put screening method to screen 146 soil bacterial isolates for antimicrobial activity in monocultures and in interactions.

Another aim of my thesis research was to investigate the effects of interspecific interactions on bacterial fitness, gene expression and on the global produced metabolome, including both soluble and volatile compounds.

Research questions related to the frequency of competition mediated triggering of antimicrobial activity in soil bacteria, the consequences of interspecific interactions on bacterial fitness, gene expression and the production of soluble and volatile secondary metabolites with inhibitory capacities are addressed in six chapters of this thesis. 
The three main hypotheses for the thesis research are:

(1) Interspecific interactions have a major effect on antimicrobial compound production including both soluble and volatile antimicrobial compounds.

(2) Interspecific interactions have a significant effect on bacterial fitness, gene expression and consequently on soil bacterial community composition.

(3) The production of antimicrobial compounds is costly.

The research questions of this thesis are:

(1): What is the frequency of interspecific triggering of antimicrobial activity in soil bacteria?

The frequency of interaction mediated triggered antimicrobial activity among soil bacteria is addressed in the second chapter. In this chapter I highlight the importance of interspecific interactions for both induction and silencing of antimicrobial activity.

(2): How important are interspecific interactions for secondary metabolite production by soil bacteria?

The impact of bacterial interspecific interactions on secondary metabolite production in bacteria is addressed in chapters three and four. These chapters report the results of research on the impact of interspecific interactions on the production of volatiles (chapter three and four) and soluble secondary metabolites (chapter four). Furthermore I evaluated the effect of interspecific interactions on gene expression and bacterial fitness (chapter four). 
(3): What is the effect of interspecific bacterial interactions on the fitness of soil bacteria?

The effect of interspecific interactions on the fitness of soil bacteria is addressed in chapter five. Here, I used a sand microcosm approach and applied the Malthusian growth model (population growth) to investigate how Pseudomonas fluorescens Pfo-1 responds to the presence of two phylogenetically different bacteria, under two different nutritional conditions.

(4): What are the possible costs for competitor induced antimicrobial compound production?

Chapter six reports on the possible biological costs for the production of an antimicrobial compound triggered through interspecific bacterial interactions (competitor induced). Here it is shown that the facultative production of antibiotics do not mitigate any metabolic costs, but might be an advantageous survival strategy because it limits the risk of competitors evolving resistance, or the risk of competitors feeding on the antimicrobial compounds.

In chapter seven I summarize and discuss the outcome of all experimental chapters of this thesis and the importance of mining into interspecific bacterial interactions. Furthermore this chapter will give a critical outlook on the future of antimicrobial discovery. 


\section{Chapter

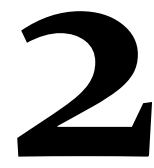

\section{Impact of interspecific interactions on antimicrobial activity among soil bacteria}

Olaf Tyc, Marlies van den Berg, Saskia Gerards, Johannes A. van Veen, Jos M. Raaijmakers, Wietse de Boer and Paolina Garbeva

This chapter has been published as:

Tyc, O., Van Den Berg, M., Gerards, S., Van Veen, J.A., Raaijmakers, J.M., De Boer, W., and Garbeva, P. (2014). Impact of interspecific interactions on antimicrobial activity among soil bacteria. Frontiers in Microbiology 5, 567. doi:10.3389/fmicb.2014.00567 


\title{
Impact of interspecific interactions on antimicrobial activity among soil bacteria
}

\begin{abstract}
Certain bacterial species produce antimicrobial compounds only in the presence of a competing species. However little is known on the frequency of interaction-mediated induction of antibiotic compound production in natural communities of soil bacteria. Here we developed a high-throughput method to screen for the production of antimicrobial activity by monocultures and pair-wise combinations of 146 phylogenetically different bacteria isolated from similar soil habitats. Growth responses of two human pathogenic model organisms, Escherichia coli WA321 and Staphylococcus aureus $533 \mathrm{R} 4$, were used to monitor antimicrobial activity. From all isolates, $33 \%$ showed antimicrobial activity only in monoculture and $42 \%$ showed activity only when tested in interactions. More bacterial isolates were active against $S$. aureus than against $E$. coli. The frequency of interaction-mediated induction of antimicrobial activity was $6 \%$ (154 interactions out of 2798) indicating that only a limited set of species combinations showed such activity.
\end{abstract}

The screening revealed also interaction-mediated suppression of antimicrobial activity for $\mathbf{2 2} \%$ of all combinations tested. Whereas all patterns of antimicrobial activity (non-induced production, induced production and suppression) were seen for various bacterial classes, interaction-mediated induction of antimicrobial activity was more frequent for combinations of Flavobacteria and alpha- Proteobacteria.

The results of our study give a first indication on the frequency of interference competitive interactions in natural soil bacterial communities which may forms a basis for selection of bacterial groups that are promising for the discovery of novel, cryptic antibiotics. 


\section{Introduction}

Production of antimicrobial compounds is an important strategy to increase competitiveness of soil bacteria. Soil is a heterogeneous, nutrientpoor and harsh environment harboring a huge diversity of bacteria (Gans et al., 2005; Uroz et al., 2010). There is also considerable functional redundancy as many soil bacterial species can use similar substrates as an energy source for growth and persistence (Yin, 2000;Strickland, 2009). Therefore, interspecific competition for nutrient resources is a major type of interaction in soil bacterial communities (Demoling et al., 2007;Rousk and Baath, 2007;Rousk et al., 2009). An important strategy in interspecific interactions, known as interference competition, is the production of growth inhibitory secondary metabolites (e.g. antibiotics, toxins, biosurfactants, volatiles and others) that can suppress or kill microbial opponents (Hibbing et al., 2010;Cornforth and Foster, 2013). Although the production of antimicrobial compounds could inhibit the growth of bacterial strains competing for resources, in some cases the produced antimicrobial compounds could also promote the growth of other bacteria (D'Costa et al., 2006;Dantas et al., 2008), act as signalling molecules (Linares et al., 2006; Romero et al., 2011) or modulate bacterial gene expression in sub inhibitory concentrations (Goh et al., 2002).

Whole genome sequencing has revealed that many soil microorganisms possess so-called cryptic gene clusters encoding for putative new secondary metabolites that are not produced during common in vitro conditions (Ikeda et al., 2003;Scherlach and Hertweck, 2009;Chiang et al., 2011;Saleh et al., 2012). In nature, however, antibiotics may be produced after perception of specific environmental signals (stress/nutrient signals) or signals from neighboring microorganisms (competitor sensing) (Firn, 2003;Cornforth and Foster, 2013;Zhu, 2014). Indeed, several studies have indicated that antibiotic production in soil bacteria can be induced when they are confronted with other bacterial species (Slattery et al., 2001;Lyon and Muir, 2003;Maurhofer et al., 2004; de Boer et al., 2007;Seyedsayamdost et al., 2012).

We hypothesize that competitor induced (facultative) rather than constitutive antibiotic production represents a key strategy in interference competition that is cost-effective and / or may reduce selection of antibiotic-resistant competitors (Garbeva et al., 2011c). Interaction-mediated induction of antibiotic production is also interesting from an applied perspective as it may lead to the discovery of novel antibiotics. 


\section{Impact of interspecific interactions on antimicrobial activity among soil bacteria}

The aim of the current study was to obtain insight in the frequency of interaction-mediated induction of antibiotic production in natural soil bacterial communities. To this end, we screened a collection of bacterial isolates obtained from similar soil habitats. We developed and applied a highthroughput method to screen bacteria for the production of compounds that inhibit growth of Gram-positive and Gram-negative isolates that are closely related to human pathogens. By selecting these target organisms the study not only revealed information on the frequency of interaction-mediated antibiotic production, but also on specific soil bacterial genera or species that could be promising candidates for the discovery of novel antibiotics.

The obtained results revealed that interactions have a major impact on antimicrobial compound production albeit with effects in both directions i.e. induction and suppression of antimicrobial activity. 


\section{Materials and methods}

\section{Soil bacteria \& culture conditions}

We selected 146 bacterial isolates from organic-poor, sandy soils under vegetation patches of sand sedge (Carex arenaria L.) growing in natural field sites (de Ridder-Duine et al., 2005) (Table S2.1). The bacterial isolates were pre-cultured from $-8 \mathrm{o}^{\circ} \mathrm{C}$ glycerol stocks on 1/10 TSBA $\left(5.0 \mathrm{gL}^{-1} \mathrm{NaCl}\right.$, $1.0 \mathrm{gL}^{-1}$ $\mathrm{KH}_{2} \mathrm{PO}_{4} ; 3 \mathrm{gL}^{-1}$ Oxoid Tryptic Soy Broth; $20 \mathrm{gL}^{-1}$ Merck Agar, pH 6.5) (Garbeva and de Boer, 2009) and incubated for 5 to 7 days at $20^{\circ} \mathrm{C}$ prior to screening.

\section{Control strains and target organisms}

Reference strains that produce known antibiotics in monoculture were obtained from the DSMZ strain collection (Leibniz Institute DSMZ-German Collection of Microorganisms and Cell Cultures, Braunschweig, Germany). These reference strains were: Streptomyces kanamyceticus (DSM 40500), producer of kanamycin, Streptomyces rimosus (DSM 40260), producer of oxytetracycline and Streptomyces nodosus (DSM 40109) producer of amphotericin $\mathrm{A}$ and $\mathrm{B}$. These strains were pre-cultured from $-80^{\circ} \mathrm{C}$ glycerol stocks on GYM agar plates (4.0 $\mathrm{gL}^{-1}$ Glucose, $4.0 \mathrm{gL}^{-1} \mathrm{BACTO}^{\mathrm{TM}}$ Yeast extract, 10.0 $\mathrm{gL}^{-1}$ Malt extract, $2.0 \mathrm{gL}^{-1} \mathrm{CaCO}_{3}$, $20 \mathrm{gL}^{-1}$ Merck Agar, $\mathrm{pH}$ 7.2) and incubated for 7 days at $28^{\circ} \mathrm{C}$ before inoculation into 96-well source plates (see below). In the agar-overlay assay, two bacterial strains were selected to act as model organisms for human pathogenic bacteria: Escherichia coli WA321 (DSM 4509) as Gram-negative target organism and Staphylococcus aureus ${ }_{533} \mathrm{R}_{4}$ Serovar 3 (DSM 20231) as Gram-positive target organism. The target strains were pre-cultured from $-8 \mathrm{o}^{\circ} \mathrm{C}$ glycerol stocks on Luria Bertani (LB) agar plates (10.0 gL ${ }^{-1} \mathrm{NaCl}, 10 \mathrm{gL}^{-1}$ Bacto $^{\mathrm{TM}}$ Tryptone, $5 \mathrm{gL}^{-1} \mathrm{Bacto}^{\mathrm{TM}}$ Yeast extract, $20 \mathrm{gL}^{-1}$ Merck Agar) Sambrook and Russell (2001) and incubated at $37^{\circ} \mathrm{C}$ for 24 $\mathrm{h}$ before inoculation in the antimicrobial screening assay. Characteristics of the target and the control strains are listed in Table S2.2.

\section{Preparation of OmniTray ${ }^{\mathrm{TM}}$ plates}

For the high-throughput interaction assay polystyrene $\mathrm{Nunc}^{\mathrm{TM}}$ OmniTray ${ }^{\mathrm{TM}}$ plates (size $128 \times 86 \mathrm{~mm}$; cap. $90 \mathrm{~mL}$; Nunc ${ }^{\mathrm{TM}}$, Nalge Nunc International, Rochester, NY, USA Cat \# 82-264728) were used. Each OmniTray ${ }^{\mathrm{TM}}$ plate was filled with $45 \mathrm{~mL}$ of $1 / 10$ TSBA ( $2 \%$ ) agar. Plates were kept in the laminar flow cabinet until the agar was completely solidified. 


\section{Impact of interspecific interactions on antimicrobial activity among soil bacteria}

\section{Preparation of 96-well source-plates}

96-well Microtiter plates (Greiner bio-one B.V., Alphen a/d Rijn, The Netherlands, Cat\# 655180) were prepared to inoculate the selected bacterial isolates and the reference strains. Each well was filled with $150 \mu$ liquid LB broth. Bacterial isolates were inoculated in 10 rows containing quadruplicates of each strain, the $11^{\text {th }}$ row was kept empty and the $12^{\text {th }}$ row was used as positive control by inoculating known antibiotic-producing Streptomyces strains in duplicate with one free well between each strain (Figure 2.1). Inoculation was done by picking cells from a single colony of each bacterial strain with a disposable inoculation loop (VWR international B.V., Amsterdam, The Netherlands Cat\# 50806-404) and transferring to the designated well in the 96-well source plates. The plates were incubated for two days at $24{ }^{\circ} \mathrm{C}$, after which the plates were prepared for long-term storage $\left(-80{ }^{\circ} \mathrm{C}\right.$ freezer) by adding $50 \mu \mathrm{l}$ of $50 \%(\mathrm{v} / \mathrm{v})$ glycerol to achieve a final concentration of $12.5 \%(\mathrm{v} / \mathrm{v})$. In total, 15 Microtiter plates (source plates A O) containing different compositions of monocultures of bacterial isolates were prepared for the high-throughput interaction assay.

\section{High-throughput interaction assay}

A Genetix QPix 2 colony-picking robot (Molecular Devices, UK Limited, Wokingham, United Kingdom) was used for the high-throughput interaction assay. The Genetix QPix 2 robot was mounted with a bacterial 96-pin picking head and programmed to replicate the source plates (96-well Microtiter plates) into the OmniTray ${ }^{\mathrm{TM}}$ plates (Figure 2.1). The source plates were replicated two times, one set of inoculated plates was removed from the robot and was used as control to estimate growth and antimicrobial activity of the monocultures. The remaining plates in the robot were used for the interaction assay by inoculating a second set of source-plates in various combinations. The second set of bacterial isolates was inoculated at the same position as the first set of bacteria, in this way the bacterial isolates had physical cell contact and could interact in one-to-one interactions (in quadruplicates). The inoculated OmniTray ${ }^{\mathrm{TM}}$ plates (monocultures and interaction plates) were incubated for 4 days at $24{ }^{\circ} \mathrm{C}$. In total, 146 bacterial isolates were combined with each other in various arrangements and tested in 2798 unique interactions for the production of antimicrobial compounds. 


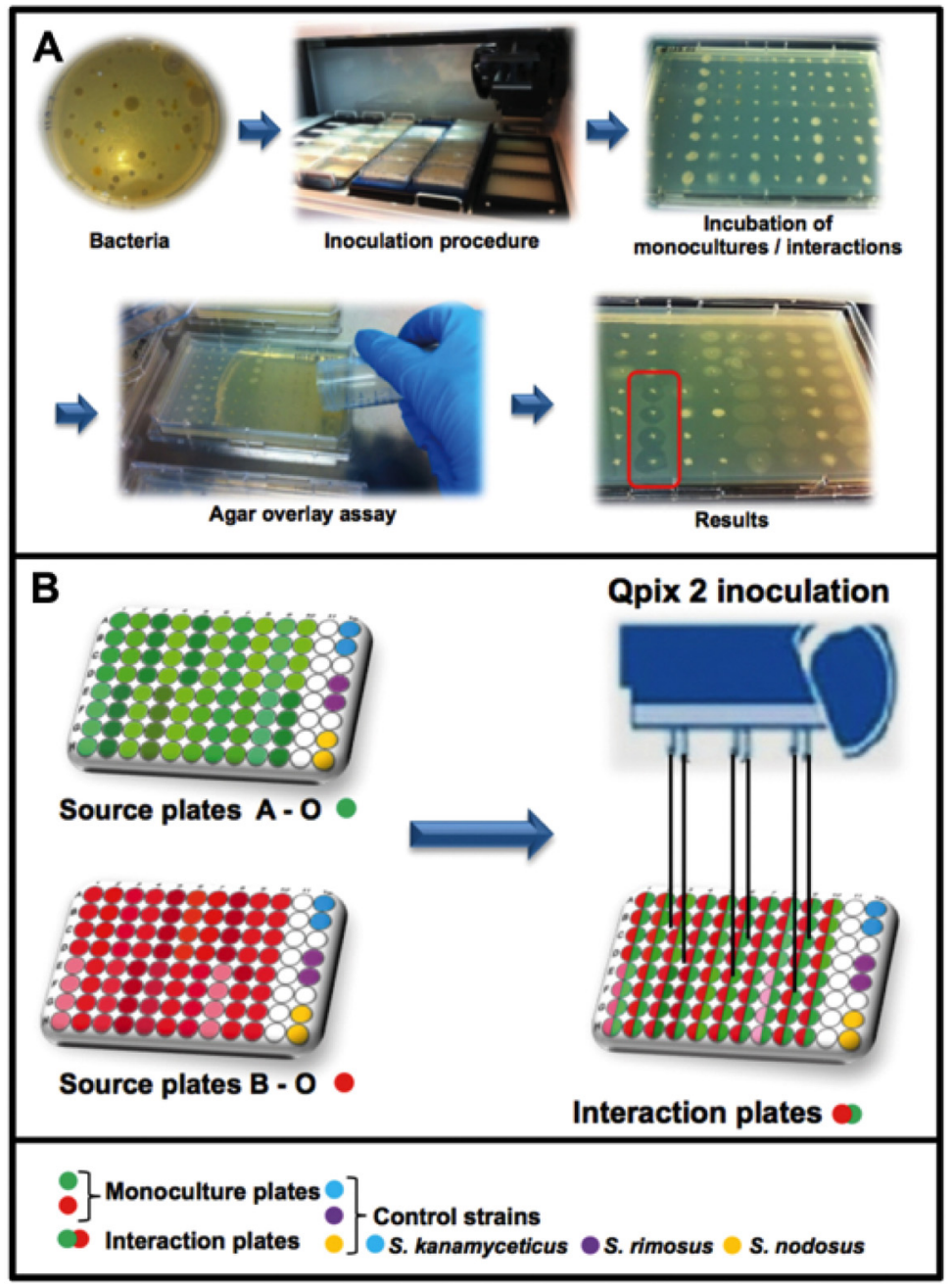

Figure 2.1: Workflow of the high-throughput interaction assay. (A) Overview of the antimicrobial screening: bacteria were inoculated with a Genetix Qpix 2 colony picking robot either in monoculture or in one-to-one interactions on OmniTray $^{\mathrm{TM}}$ plates. For the detection of antimicrobial activity an agar overlay assay with two target organisms was performed on the fourth day of incubation. Antimicrobial activity was determined on the $5^{\text {th }}$ day after overnight incubation at $37^{\circ} \mathrm{C}$ by screening for visible zones of inhibition (ZOI) in the upper agar layer. (B) Overview of the 96-well plates design and the inoculation procedure using the Genetix QPix2 colony-picking robot. 


\section{Impact of interspecific interactions on antimicrobial activity among soil bacteria}

\section{Antimicrobial screening}

For detection of antimicrobial activity, an agar overlay assay was performed on the 4th day of incubation (Nkanga and Hagedorn, 1978). The two target organisms Escherichia coli WA321 and Staphylococcus aureus 533R4 were grown overnight in liquid LB broth at $37^{\circ} \mathrm{C}$, 220 rpm. Fresh LB- agar $(1.5 \%$ Merck Agar) was prepared, cooled down to $\sim 45{ }^{\circ} \mathrm{C}$ and the target organisms were added to a final $\mathrm{OD}_{600}$ of 0.002 corresponding to approximately $6 \times \mathrm{x}^{\wedge} 5$ $\mathrm{CFU} / \mathrm{mL}$ (E.coli WA321) or $4 \times 10^{\wedge} 5 \mathrm{CFU} / \mathrm{mL}$ (S. aureus $\left.533 \mathrm{R}_{4}\right)$ and mixed well. A volume of $15 \mathrm{~mL}$ liquid LB-agar containing the target organisms was poured over the OmniTray ${ }^{\mathrm{TM}}$ plates with the empty $11^{\text {th }}$ row as the start position for pouring. After solidification of the overlay agar, the OmniTray ${ }^{\mathrm{TM}}$ plates were incubated overnight at $37^{\circ} \mathrm{C}$. The next day $\left(5^{\text {th }}\right.$ day), plates were examined for visible zones of inhibition (ZOI). Monocultures or mixed-cultures of the soil bacterial isolates were scored as positive for antibiotic production if at least two out of four replicates produced zones of inhibition (Figure 2.1A). The majority of activity reported $(>55 \%)$ involved $\geq 3$ out of 4 replicates. For confirmations of the high-throughput screening results, several of the antibiotic-triggering/suppressing interactions were tested outside the HTS setup (Figure S2.3 and S2.4).

\section{PCR and 16S rRNA gene sequencing}

For identification of the bacterial isolates, PCRs were performed directly on colonies or with extracted genomic DNA. For genomic DNA extraction the QIAGEN QIAmp DNA Mini Kit (QIAGEN Benelux B.V., Venlo, The Netherlands cat\# 51 304) was applied according to the manufacturer's manual. For the colony PCRs, a few colonies of each bacterial isolate were scraped from the plate with a disposable inoculation loop (VWR international B.V., Amsterdam, The Netherlands Cat\# 50806-404) and re-suspended in $250 \mu \mathrm{l}$ sterile MQ-water. The re-suspended bacterial cells were pulse vortexed and heated to $95{ }^{\circ} \mathrm{C}$ for $5 \mathrm{~min}$. Tubes were centrifuged for $3 \mathrm{~min}$ at 12,0oox $\mathrm{g}$ and $1 \mu \mathrm{l}$ supernatant from each bacterial isolate was applied in a $50 \mu \mathrm{l}$ PCR-master mix (Promega Corp. Madison, USA cat\# M7505). For 16S rRNA gene amplification, one of the two primer combinations was used: (1) forward primer pA (5'- AGA GTT TGA TCC TGG CTC AG -3'), reverse primer 1492r (5'GRT ACC TTG TTA CGA CTT -3'), amplifying 1492 bp from the 16S rRNA gene or (2) forward primer 27f (5'-AGA GTTT GAT CMT GGC TCAG -3'), reverse primer 1492r amplifying $\sim 1465$ bp from the $16 \mathrm{~S}$ rRNA gene (Edwards et al., 1989;Lane, 1991) (modified). All PCR reactions were performed on a MJ 
Research Peltier thermal cycler 200 PCR machine (Harlow Scientific, Arlington, USA) with the following settings: initial cycle $95{ }^{\circ} \mathrm{C}$ for $5 \mathrm{~min}$. and 30 cycles of $94{ }^{\circ} \mathrm{C}$ for 30 sec., $55{ }^{\circ} \mathrm{C}$ for 30 sec. and $72{ }^{\circ} \mathrm{C}$ for $1 \mathrm{~min}$. After amplification, a volume of $5 \mu$ of each PCR reaction was loaded on a $1.25 \%$ $(\mathrm{w} / \mathrm{v})$ agarose gel and checked after electrophoresis for presence of PCR fragment. The PCR products were sent to MACROGEN (MACROGEN Europe, Amsterdam, The Netherlands) for sequencing.

\section{Phylogenetic analysis and sequence analysis}

Obtained sequence chromatograms of the 16S rRNA gene were examined for quality and trimmed to approximately the same size ( 650 bp) using 4 PEAKS V1.7.2 for MAC OS X (www.nucleobytes.com) (C) 2006 Mek\&Tosj.com and Clustal W. The aligned 16S rRNA gene sequences were compared against those available in the NCBI database by BLASTN (blast.ncbi.nlm.nih.gov) (Altschul et al., 1997). The sequences obtained during this study are deposited in NCBI GenBank under accession numbers KJ685218 - KJ685361. For two isolates, the $16 \mathrm{~S}$ rRNA sequences were available from previous work: P. fluorescens (strain AD21): DQ778036, Pedobacter sp. (strain V48): DQ778037 (de Boer et al., 2007).

\section{Network visualization of interactions}

The bacterial interaction pairs that triggered or suppressed antimicrobial activity against the target organisms were visualized with Cytoscape 3.0.2 (www.cytoscape.org) for MAC OS X (Shannon et al., 2003). Interaction visualizations were performed with the following parameters: each phylogenetic class was visualized as a single node with different symbols for each phylogenetic class, the interactions between the phylogenetic classes (nodes) were visualized by links (edges) connecting each interacting phylogenetic class. Node colours were scaled to the number of interactions between the different phylogenetic classes (see Figure legends). For visualization, self-loops (interactions within the same phylogenetic class) and edges (interactions between phylogenetic classes) were bundled to single links between the respective phylogenetic classes (the darker the line the higher the number of interactions between the phylogenetic classes). 


\section{Impact of interspecific interactions on antimicrobial activity among soil bacteria}

\section{Statistical analysis}

Statistical analyses on frequencies for induction and/or suppression of antimicrobial compound production between the different Gram-groups were performed with http://math.hws.edu/javamath/ryan/ChiSquare.html using online chi-square tests. Results of the chi-square test are shown in Table S2.5 and S2.6.

\section{Results}

\section{Phylogeny of the tested bacterial isolates}

$16 \mathrm{~S}$ rRNA gene sequence analysis revealed that the 146 bacterial isolates tested in this study belonged to 4 phyla covering 7 classes and 9 genera: Proteobacteria (14 alpha- Proteobacteria, 65 beta -Proteobacteria, 29 gammaProteobacteria), Bacteroidetes (19 Flavobacteria, 1 Sphingobacteria), Actinobacteria (11 Actinobacteria) and Firmicutes (7 Bacilli) (Table 2.1 and S2.1).

Table 2.1: Frequencies of antimicrobial activity for the phyla included in this study.

\begin{tabular}{|c|c|c|c|c|c|}
\hline $\begin{array}{c}\text { Phylum / } \\
\text { phylogenetic class }\end{array}$ & $\begin{array}{c}\text { Total } \\
\text { abundance }\end{array}$ & $\begin{array}{c}\text { AM active vs } \\
\begin{array}{c}\text { E. coli in } \\
\text { monoculture }\end{array}\end{array}$ & $\begin{array}{c}\text { AM active vs } \\
\text { E. coli in } \\
\text { interaction }\end{array}$ & $\begin{array}{c}\text { AM active vs } \\
S . \text { aureus in } \\
\text { monoculture }\end{array}$ & $\begin{array}{l}\text { AM active vs } \\
S . \text { aureus in } \\
\text { interaction }\end{array}$ \\
\hline \multicolumn{6}{|l|}{ Actinobacteria } \\
\hline Actinobacteria & 11 & 3 & 3 & 4 & 5 \\
\hline \multicolumn{6}{|l|}{ Bacteroidetes } \\
\hline Flavobacteria & 19 & 1 & 3 & 3 & 11 \\
\hline Sphingobacteria & 1 & & 1 & & \\
\hline \multicolumn{6}{|l|}{ Firmicutes } \\
\hline Bacilli & 7 & 2 & 2 & 3 & 2 \\
\hline \multicolumn{6}{|l|}{ Proteobacteria } \\
\hline a-proteobacteria & 14 & & 1 & 3 & 9 \\
\hline$\beta$-proteobacteria & 65 & 17 & 8 & 26 & 25 \\
\hline$\gamma$-proteobacteria & 29 & 2 & 2 & 12 & 7 \\
\hline (n) isolates & 146 & 25 & 20 & 51 & 59 \\
\hline
\end{tabular}




\section{High-throughput screening for antimicrobial activity}

We developed a high-throughput assay to screen for production of antimicrobial compounds by interacting bacteria (Figure 2.1). In total 146 isolates were screened in monocultures and in 2798 random one-to-one interactions. For 17 isolates (11 \%), no activity against $E$. coli and $S$. aureus was detected not in monocultures nor in mixed cultures (Table S2.7 and Figure 2.2A). For 20 isolates (14\%) antibacterial activity was observed in both monoculture and mixed cultures. For 48 isolates (33\%), this was restricted to monocultures only and for 61 isolates (42\%) antibacterial activity was only apparent during interactions (Figure 2.2A and Figure 2.3).

The number of isolates (110) involved in activity against the Gram-positive target strain S. aureus $533 \mathrm{R}_{4}$ was more than twice the number of isolates (45) with activity against the Gram-negative target strain E. coli WA321 (Table 2.1, Table S2.1). Despite the high number of bacterial isolates involved in antimicrobial activity in interactions, the frequency of interaction-mediated induction of antimicrobial activity was low $\sim 6 \%$ (154 interactions out of 2798). This implies that interaction-mediated induction was only occurring in a limited number of combinations (Table 2.2 and Table 2.3).

Most interactions (72 \%) did not have an effect on antimicrobial activity (induction or suppression) and about $22 \%$ of the interactions suppressed antimicrobial activity in isolates that revealed activity in monoculture (Figure 2.2B). 


\section{Impact of interspecific interactions on antimicrobial activity among soil bacteria}

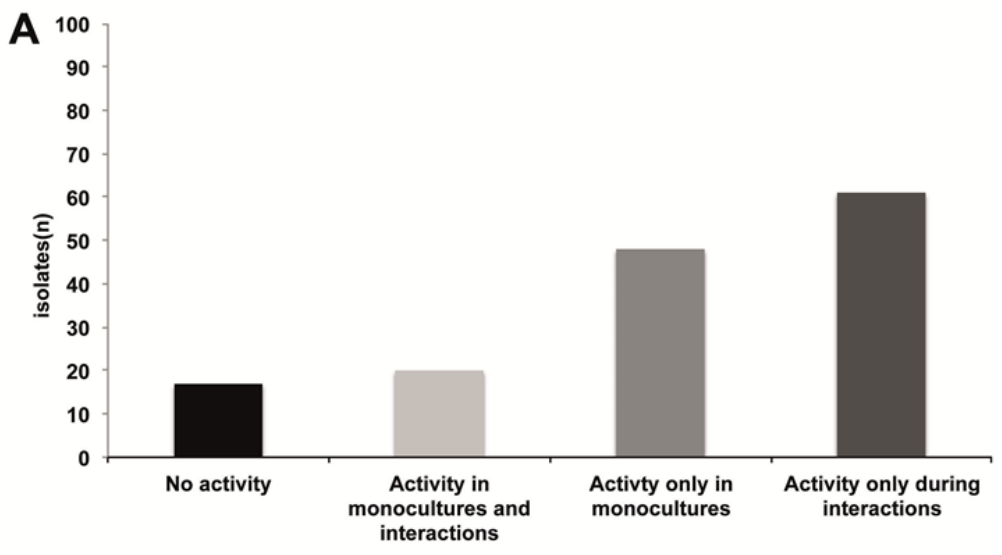

B

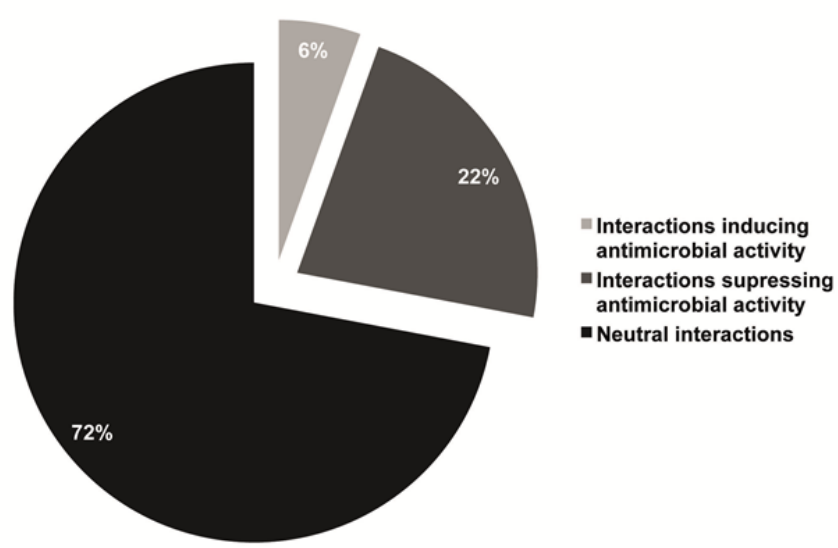

Figure 2.2: (A) Number of bacterial isolates exhibiting different patterns of antimicrobial activity against E.coli WA321 and / or S.aureus $533 \mathrm{R}_{4}$; in total 146 bacterial isolates were studied (B) Frequencies of interactions (1) inducing antimicrobial activity, (2) suppressing antimicrobial activity and (3) neutral interactions (no induction/suppression). Number of tested combinations $(n=2798)$. 


\section{Antimicrobial activity during interactions}

\section{Interaction-mediated activity against $E$. coli WA321}

Growth of E.coli WA321 was inhibited by 14 pair-wise combinations involving 20 isolates that did not show antimicrobial activity in monoculture (Table 2.2). Some isolates were present in different combinations. For example, Janthinobacterium sp. AD8o and Streptomyces sp. AD1o8 were present in 4 combinations with induced activity (Table 2.2). Combinations inhibiting growth of E. coli WA321 consisted of Gram-negative/Gram-positive isolates (7 interactions) or Gram-negative/Gram-negative (6 interactions). Only in one case, a combination of two Gram-positive isolates (Micrococcus \& Microbacterium) showed activity against E.coli.

Table 2.2: Bacterial pairs with induced antimicrobial activity against E.coli WA 321.

\begin{tabular}{|c|c|c|c|}
\hline Phylogenetic class & Genus A & Phylogenetic class & Genus B \\
\hline alpha-proteobacteria & Phyllobacterium sp. AD152 & gamma-proteobacteria & Pseudomonas sp. AD114 \\
\hline beta-proteobacteria & Burkholderia sp. $\mathrm{AD}_{24}$ & beta-proteobacteria & Collimonas sp. AD68 \\
\hline beta-proteobacteria & Burkholderia sp. $\mathrm{AD}_{32}$ & beta-proteobacteria & Janthinobacterium sp. AD8o \\
\hline beta-proteobacteria & Janthinobacterium sp. $\mathrm{AD}_{72}$ & Flavobacteria & Flavobacterium sp. AD151 \\
\hline beta-proteobacteria & Janthinobacterium sp. AD8o & gamma-proteobacteria & Dyella sp. $\mathrm{AD}_{5} 6$ \\
\hline beta-proteobacteria & Janthinobacterium sp. AD8o & beta-proteobacteria & Variovorax sp. AD133 \\
\hline Actinobacteria & Streptomyces sp. AD1o8 & beta-proteobacteria & Burkholderia sp. $\mathrm{AD}_{37}$ \\
\hline Actinobacteria & Streptomyces sp. AD1o8 & Flavobacteria & Flavobacterium sp. AD47 \\
\hline Actinobacteria & Streptomyces sp. AD1o8 & Flavobacteria & Flavobacterium sp. AD84 \\
\hline Actinobacteria & Streptomyces sp. AD1o8 & Sphingobacteria & Pedobacter sp. $\mathrm{V}_{4} 8$ \\
\hline Actinobacteria & Microbacterium sp. AD141 & beta-proteobacteria & Janthinobacterium sp. AD8o \\
\hline Bacilli & Bacillus sp. $\mathrm{AD}_{7} 8$ & beta-proteobacteria & Burkholderia sp. AD11 \\
\hline Bacilli & Paenibacillus sp. AD83 & beta-proteobacteria & Burkholderia sp. AD24 \\
\hline Actinobacteria & Micrococcus sp. $\mathrm{AD}_{31}$ & Actinobacteria & Microbacterium sp. AD141 \\
\hline
\end{tabular}

\section{Interaction-mediated activity against $S$. aureus $533 R_{4}$}

Growth of $S$. aureus $533 \mathrm{R}_{4}$ was inhibited by 63 pair-wise combinations involving 59 isolates. Several isolates were present in multiple combinations that inhibited growth of $S$. aureus (Table 2.3). Burkholderia sp. AD 37, Collimonas sp. AD65, Collimonas sp. AD98, Janthinobacterium sp. AD72, Micrococcus sp. AD31, Pseudomonas sp. AD104, Streptomyces spp. AD92 and AD1o8, Variovorax sp. AD143 were all involved in more than five combinations that inhibited the growth of $S$. aureus. Most of the combinations consisted of Gram-negative/Gram-negative isolates (35 interactions) or Gramnegative/Gram-positive isolates (25 interactions). Activity against $S$. aureus was only observed 3 times for Gram-positive/Gram-positive combinations (Figure S2.8). 


\section{Impact of interspecific interactions on antimicrobial activity among soil bacteria}

Table 2.3: Bacterial pairs with induced antimicrobial activity against $S$. aureus

$533 R_{4}$.

\begin{tabular}{|c|c|c|c|}
\hline Phylogenetic class & Genus A & Phylogenetic class & Genus B \\
\hline alpha-proteobacteria & Phyllobacterium sp. $\mathrm{AD}_{34}$ & beta-proteobacteria & Collimonas sp. AD89 \\
\hline alpha-proteobacteria & Phyllobacterium sp. AD153 & beta-proteobacteria & Collimonas sp. AD65 \\
\hline beta-proteobacteria & Collimonas sp. AD69 & Flavobacteria & Flavobacterium sp. $\mathrm{AD}_{43}$ \\
\hline beta-proteobacteria & Janthinobacterium sp. $\mathrm{AD}_{72}$ & gamma-proteobacteria & Dyella sp. $\mathrm{AD}_{4} 6$ \\
\hline beta-proteobacteria & Janthinobacterium sp. $\mathrm{AD}_{72}$ & beta-proteobacteria & Collimonas sp. AD97 \\
\hline beta-proteobacteria & Janthinobacterium sp. $\mathrm{AD}_{72}$ & alpha-proteobacteria & Agrobacterium sp. AD140 \\
\hline beta-proteobacteria & Collimonas sp. AD61 & beta-proteobacteria & Collimonas sp. AD98 \\
\hline beta-proteobacteria & Collimonas sp. AD67 & beta-proteobacteria & Collimonas sp. AD68 \\
\hline beta-proteobacteria & Janthinobacterium sp. $\mathrm{AD}_{75}$ & beta-proteobacteria & Burkholderia sp. $\mathrm{AD}_{37}$ \\
\hline beta-proteobacteria & Collimonas sp. AD69 & Flavobacteria & Flavobacterium sp. AD146 \\
\hline beta-proteobacteria & Collimonas sp. $\mathrm{AD}_{71}$ & alpha-proteobacteria & Rhizobium sp. AD148 \\
\hline beta-proteobacteria & Collimonas sp. AD88 & beta-proteobacteria & Burkholderia sp. $\mathrm{AD}_{37}$ \\
\hline beta-proteobacteria & Collimonas sp. AD102 & Flavobacteria & Flavobacterium sp. $\mathrm{AD}_{45}$ \\
\hline beta-proteobacteria & Collimonas sp. AD98 & Flavobacteria & Flavobacterium sp. AD142 \\
\hline beta-proteobacteria & Burkholderia sp. $\mathrm{AD}_{37}$ & gamma-proteobacteria & Pseudomonas sp. AD104 \\
\hline beta-proteobacteria & Collimonas sp. AD99 & beta-proteobacteria & Burkholderia sp. AD138 \\
\hline beta-proteobacteria & Collimonas sp. AD89 & alpha-proteobacteria & Mesorhizobium sp. $\mathrm{AD}_{3} 8$ \\
\hline beta-proteobacteria & Variovorax sp. AD143 & beta-proteobacteria & Collimonas sp. AD65 \\
\hline beta-proteobacteria & Variovorax sp. AD143 & alpha-proteobacteria & Mesorhizobium sp. AD112 \\
\hline beta-proteobacteria & Variovorax sp. AD143 & alpha-proteobacteria & Phyllobacterium sp. AD153 \\
\hline beta-proteobacteria & Collimonas sp. AD98 & alpha-proteobacteria & Phyllobacterium sp. AD159 \\
\hline beta-proteobacteria & Collimonas sp. AD98 & gamma-proteobacteria & Pseudomonas sp. AD105 \\
\hline beta-proteobacteria & Collimonas sp. AD137 & gamma-proteobacteria & Pseudomonas sp. AD157 \\
\hline beta-proteobacteria & Collimonas sp. AD97 & beta-proteobacteria & Collimonas sp. AD62 \\
\hline beta-proteobacteria & Roseateles sp. AD145 & beta-proteobacteria & Collimonas sp. AD67 \\
\hline gamma-proteobacteria & Pseudomonas sp. AD124 & beta-proteobacteria & Collimonas sp. AD65 \\
\hline gamma-proteobacteria & Pseudomonas sp. AD114 & beta-proteobacteria & Burkholderia sp. AD18 \\
\hline gamma-proteobacteria & Pseudomonas sp. AD105 & alpha-proteobacteria & Bosea sp. AD132 \\
\hline gamma-proteobacteria & Pseudomonas sp. AD104 & alpha-proteobacteria & Phyllobacterium sp. $\mathrm{AD}_{136}$ \\
\hline gamma-proteobacteria & Pseudomonas sp. AD104 & Flavobacteria & Chryseobacterium sp. $\mathrm{AD}_{4} 8$ \\
\hline Flavobacteria & Flavobacterium sp. AD91 & beta-proteobacteria & Variovorax sp. AD143 \\
\hline Flavobacteria & Flavobacterium sp. AD91 & alpha-proteobacteria & Phyllobacterium sp. AD153 \\
\hline Flavobacteria & Flavobacterium sp. $\mathrm{AD}_{42}$ & Flavobacteria & Flavobacterium sp. AD146 \\
\hline Flavobacteria & Flavobacterium sp. AD155 & beta-proteobacteria & Collimonas sp. AD98 \\
\hline Flavobacteria & Flavobacterium sp. $\mathrm{AD}_{44}$ & beta-proteobacteria & Collimonas sp. AD62 \\
\hline Actinobacteria & Micrococcus sp. $\mathrm{AD}_{31}$ & beta-proteobacteria & Collimonas sp. AD65 \\
\hline Actinobacteria & Micrococcus sp. $\mathrm{AD}_{31}$ & beta-proteobacteria & Collimonas sp. AD69 \\
\hline Actinobacteria & Micrococcus sp. $\mathrm{AD}_{31}$ & beta-proteobacteria & Collimonas sp. $\mathrm{AD}_{70}$ \\
\hline Actinobacteria & Micrococcus sp. $\mathrm{AD}_{31}$ & Flavobacteria & Flavobacterium sp. AD8 5 \\
\hline Actinobacteria & Micrococcus sp. $\mathrm{AD}_{31}$ & beta-proteobacteria & Collimonas sp. AD88 \\
\hline Actinobacteria & Micrococcus sp. $\mathrm{AD}_{31}$ & alpha-proteobacteria & Phyllobacterium sp. AD136 \\
\hline Actinobacteria & Micrococcus sp. $\mathrm{AD}_{31}$ & gamma-proteobacteria & Stenotrophomonas sp. AD147 \\
\hline Actinobacteria & Micrococcus sp. $\mathrm{AD}_{31}$ & Flavobacteria & Flavobacterium sp. AD156 \\
\hline Actinobacteria & Streptomyces sp. AD92 & beta-proteobacteria & Collimonas sp. AD65 \\
\hline Actinobacteria & Streptomyces sp. AD92 & beta-proteobacteria & Variovorax sp. AD143 \\
\hline Actinobacteria & Streptomyces sp. AD92 & beta-proteobacteria & Burkholderia sp. AD18 \\
\hline Actinobacteria & Streptomyces sp. AD92 & alpha-proteobacteria & Phyllobacterium sp. AD153 \\
\hline Actinobacteria & Tsukamurella sp. AD1o6 & beta-proteobacteria & Collimonas sp. AD89 \\
\hline Actinobacteria & Tsukamurella sp. AD1o6 & Flavobacteria & Chryseobacterium sp. $\mathrm{AD}_{4} 8$ \\
\hline Actinobacteria & Streptomyces sp. AD1o8 & beta-proteobacteria & Burkholderia sp. $\mathrm{AD}_{37}$ \\
\hline Actinobacteria & Streptomyces sp. AD1o8 & Flavobacteria & Chryseobacterium sp. $\mathrm{AD}_{4} 8$ \\
\hline Actinobacteria & Streptomyces sp. AD1o8 & beta-proteobacteria & Janthinobacterium sp. $\mathrm{AD}_{73}$ \\
\hline Actinobacteria & Streptomyces sp. AD1o8 & beta-proteobacteria & Janthinobacterium sp. $\mathrm{AD}_{75}$ \\
\hline Actinobacteria & Streptomyces sp. AD1o8 & beta-proteobacteria & Collimonas sp. AD88 \\
\hline Actinobacteria & Streptomyces sp. AD1o8 & beta-proteobacteria & Collimonas sp. AD1o1 \\
\hline Actinobacteria & Streptomyces sp. AD1o8 & gamma-proteobacteria & Pseudomonas sp. ADio4 \\
\hline Actinobacteria & Microbacterium sp. AD141 & beta-proteobacteria & Burkholderia sp. $\mathrm{AD}_{37}$ \\
\hline Bacilli & Paenibacillus sp. AD83 & beta-proteobacteria & Collimonas sp. AD62 \\
\hline Bacilli & Paenibacillus sp. AD8 3 & beta-proteobacteria & Burkholderia sp. $\mathrm{AD}_{24}$ \\
\hline Bacilli & Paenibacillus sp. AD116 & gamma-proteobacteria & Pseudomonas sp. ADio4 \\
\hline Actinobacteria & Micrococcus sp. $\mathrm{AD}_{31}$ & Actinobacteria & Tsukamurella sp. AD1o6 \\
\hline Actinobacteria & Tsukamurella sp. AD1o6 & Actinobacteria & Microbacterium sp. AD141 \\
\hline Actinobacteria| & Streptomyces sp. AD1o8 & Actinobacteria & Microbacterium sp. AD141 \\
\hline
\end{tabular}




\section{Interaction-mediated activity against both target organisms}

Nine isolates were present in pair-wise combinations that exhibited antimicrobial activity against both target organisms (Table S2.1). Two combinations were inhibitory for both target organisms. These were the combinations of Burkholderia sp. AD24 and Paenibacillus sp. AD83 (Figure 2.3) and of Streptomyces sp. AD1o8 and Burkholderia sp. AD37.

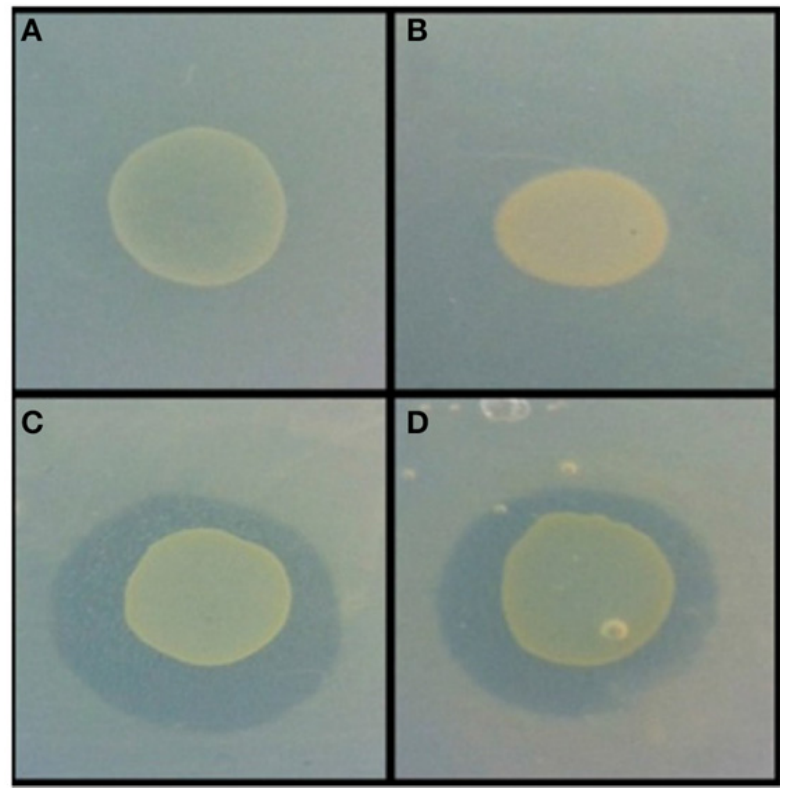

Figure 2.3: Example of antimicrobial activity revealed via the agar overlay assay: Burkholderia sp. AD24 monoculture (A), Paenibacillus sp. AD83 monoculture (B), Interaction Burkholderia sp. AD24 with Paenibacillus sp. AD83 antimicrobial activity against $S$. aureus $533 R_{4}$ (C) and antimicrobial activity against $E$. coli WA321 (D). 


\section{Impact of interspecific interactions on antimicrobial activity among soil bacteria}

Interactions inducing antimicrobial activity against $\boldsymbol{E}$.coli or $\boldsymbol{S}$.aureus

The number of pair-wise combinations with induced antimicrobial activity against $S$. aureus $533 \mathrm{R}_{4}$ was higher than against E. coli WA321. Most combinations with induced activity against E. coli WA321 involved betaProteobacteria, Actinobacteria, Flavobacteria and Bacilli (Figure 2.4A). Combinations with induced activity against $S$. aureus $533 \mathrm{R}_{4}$ involved all classes of Proteobacteria, Actinobacteria, Flavobacteria, and Bacilli (Figure 2.4B). Two phylogenetic classes, Flavobacteria and alpha- Proteobacteria, were 3 times more represented in pair-wise combinations with antimicrobial activity than in monocultures (Table 2.1).

Interactions suppressing antimicrobial activity against E.coli or

\section{S.aureus}

$22 \%$ of the isolates with antimicrobial activity in monoculture lost this activity during interactions. This apparent suppression of antimicrobial activity was found among all bacterial classes included in this study (Figure 2.5A, 2.5B). Suppression of antimicrobial activity was more frequently found for $S$. aureus than for E. coli (Figure 2.3B). The lists of bacterial pairs that suppressed antimicrobial activity against S.aureus and/or E.coli are shown in Table S2.9 and S2.10. 

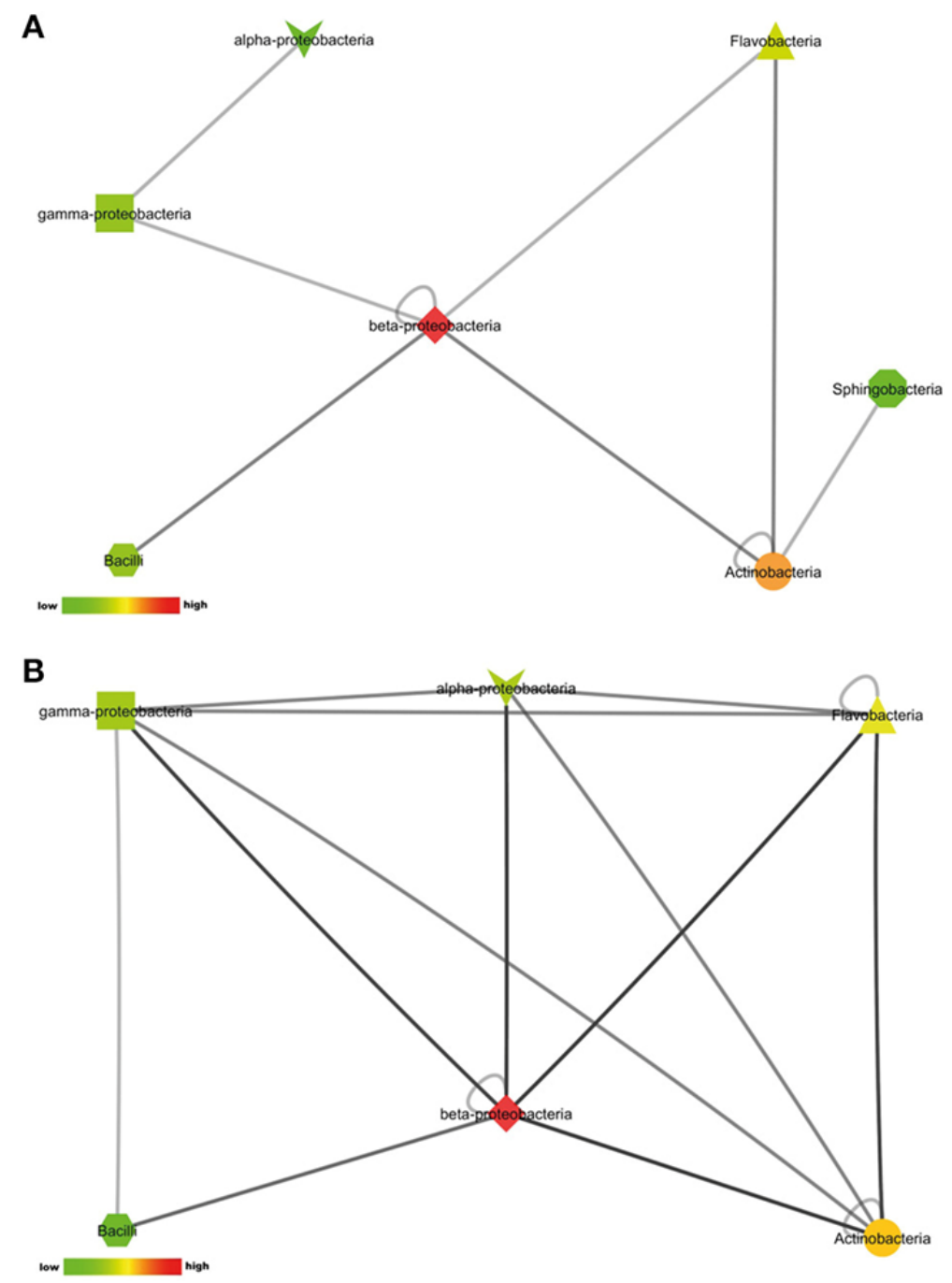

Figure 2.4: Interactions between phylogenetic classes that induced antimicrobial activity against (A) the Gram-negative target organism E.coli WA321, or (B) against the Gram-positive target organism S.aureus $533 \mathrm{R} 4$. Node colours are scaled to the number of interactions between the phylogenetic classes, low number of interactions in bright green, high number of interactions in dark red (see colour bar). 


\section{Impact of interspecific interactions on antimicrobial activity}

among soil bacteria
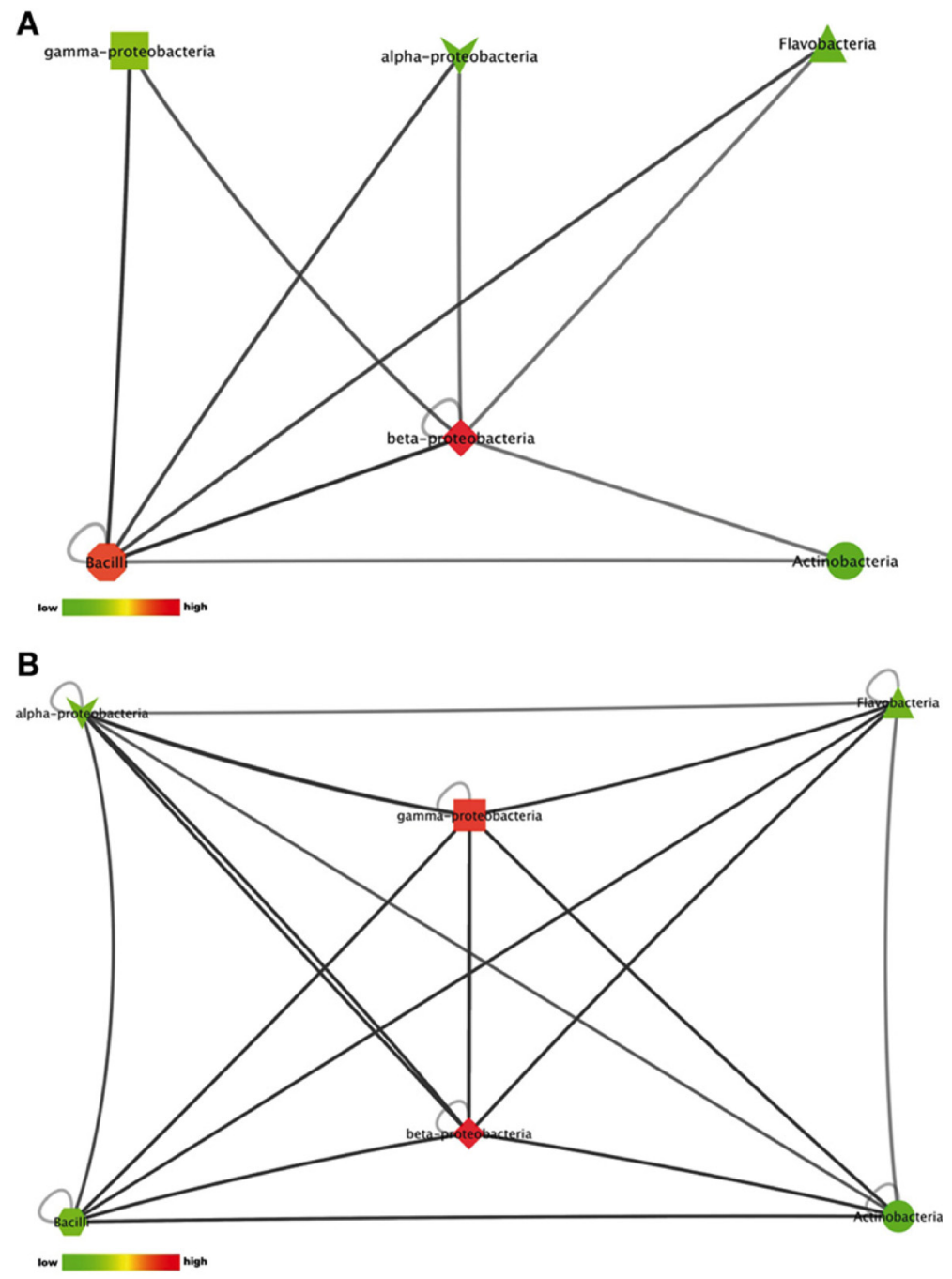

Figure 2.5: Interactions between phylogenetic classes that inhibited antimicrobial activity against (A) the Gram-negative target organism E.coli WA321, or (B) against the Gram-positive target organism S.aureus 533R4. Node colours are scaled to the respective number of interactions between the phylogenetic classes (low number of interactions in bright colours, high number of interactions in dark colours). 


\section{Discussion}

Recent studies indicated the importance of interspecific bacterial interactions for triggering antibiotic production (Garbeva et al., 2011b;Seyedsayamdost et al., 2012). However, the frequency of such events in natural bacterial communities is not known. Our study focused on a collection of bacterial isolates from similar soil habitats, i.e. sandy soils covered by vegetation patches consisting of sand sedge (Carex arenaria). Hence, the chance that actual interactions between these bacteria can occur in their natural habitat seems plausible. Induction of antibiotic production in pair-wise combinations was not found to be an abundant phenomenon as it occurred in $\sim 6 \%$ of all interactions studied. Yet, $42 \%$ of the bacterial isolates were present in combinations that showed activity against at least one of the target organisms, whereas they did not show activity in monocultures. This seems to indicate that the composition of the interacting pairs is an important factor in the induction of antibiotic production.

The observed frequency of interaction-mediated induction of antibiotic production exemplifies that a high-throughput screening as the one developed here can be an important strategy for the discovery of novel cryptic antibiotics. Many pair-wise combinations have to be screened and, subsequently, interesting pairs can be studied in more detail with respect to elucidate the mechanisms underlying the induction, signals and genes involved in the production of the antibiotic compounds (Garbeva et al., 2011b;Traxler et al., 2013). Interactions that induced antimicrobial activity often involved combinations of phylogenetically different bacteria or interactions among beta-Proteobacteria and among Actinobacteria. The present work included several bacterial genera (e.g. Streptomyces, Burkholderia, Janthinobacterium and Paenibacillus) for which multiple antibiotics have been described previously (Pantanella et al., 2007;Berdy, 2012; Cornforth and Foster, 2013;Debois et al., 2013;Zhu, 2014). Hence, there is the possibility that our screening method will reveal bacteria that produce known antibiotics but only during co-cultivation.

Few bacterial isolates of the classes Flavobacteria and alpha- Proteobacteria showed antimicrobial activity in monoculture, whereas several strains were present in antibiotic producing combinations. Hence, for these groups there is a clear potential to discover novel antibiotics. Of the 146 tested isolates, $33 \%$ showed antimicrobial activity in monoculture. This obtained frequency is in 


\section{Impact of interspecific interactions on antimicrobial activity among soil bacteria}

line with previous studies on frequencies of antimicrobial activity in Streptomyces spp. (Davelos et al., 2004;Kinkel et al., 2014). However, in many cases antibiotic production was lost when the strain was combined with another strain and only a small percentage (13\%) kept their antimicrobial activity in both combinations and monoculture. This suppressing effect on antibiotic production was more often found ( $22 \%$ of all combinations) than the induction of antibiotic production ( $\sim 6 \%$ of all combinations). Several mechanisms can be responsible for the observed suppression of antimicrobial activity during interactions e.g. interference with the quorum sensing system or other signal transduction pathways involved in regulating antibiotic production (Gonzalez and Keshavan, 2006;Venturi and Subramoni, 2009; Christensen, 2013) or direct growth inhibition of the antibiotic producing strain (Straight et al., 2007;Hibbing et al., 2010;Schneider et al., 2012). Another possible reason for the observed inhibition of antimicrobial activity during interactions could be lower nutrient availability for each strain during co-cultivation. Growth conditions and nutrient availability are important factors affecting the production of antimicrobial compounds in bacteria (van Wezel and McDowall, 2011). Antibiotic resistance mechanisms might also play a role in the observed inhibition of antimicrobial activity during co-cultivation (Rice, 2006;Wellington et al., 2013).

Depending on the target organism there was a clear difference in antimicrobial activity with higher activity against the Gram-positive than against the Gram-negative organism (in both monocultures and interactions), which is in line with previous reports that Gram-positive bacteria are generally more sensitive to antibiotics (Rice, 2006;Giske et al., 2008;Zhu, 2014).

Soil and rhizosphere are environments where bacteria evolved the ability to produce antibiotics as competitive tool for their survival (Hibbing et al., 2010). Root-associated bacteria with antimicrobial potential play an important role in plant health (Raaijmakers and Mazzola, 2012) and understanding microbial interactions affecting antimicrobial activity may be helpful in understanding the functions and mechanisms of microbial communities contributing to plant protection. 
The knowledge obtained here could help in selecting the right players in microbial consortia and as suggested by Mendes (Mendes et al., 2013) to design "a minimal microbiome" that comprises a set of microorganisms needed to fulfill a specific ecosystem services like e.g. disease suppression.

In conclusion, the high-throughput screening method developed in this work allows for a fast detection of interaction-mediated induction or suppression of antibiotic production in soil bacteria. Such screening also allows for a better insight into different interference competitive strategies that are operational in microbial communities. This knowledge in turn can be used for construction of synthetic microbial communities (Shong et al., 2012;De Roy et al., 2013;Grosskopf and Soyer, 2014).

\section{Acknowledgment}

The BE-Basic Foundation supported this work. P.G is financed by The Netherlands Organization for Scientific Research (NWO) MEERVOUD personal grant (836.09.004). This is publication 5682 of the NIOO-KNAW. 


\section{Impact of interspecific interactions on antimicrobial activity among soil bacteria}

\section{Supplementary material}

Table S2.1: Results of antimicrobial screening in confrontation assays for all bacterial isolates used in this study.

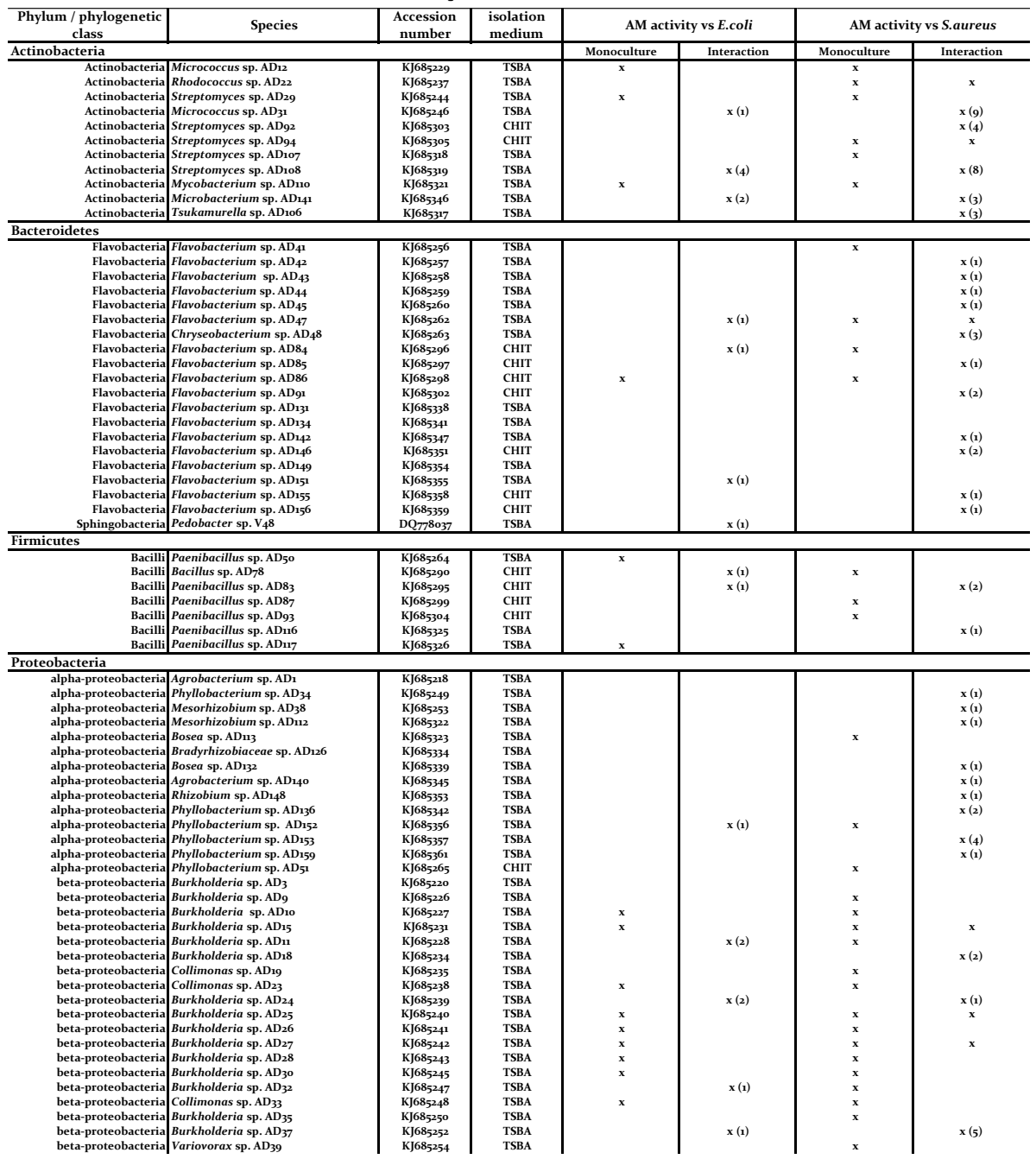




\begin{tabular}{|c|c|c|c|c|c|c|c|}
\hline $\begin{array}{c}\text { Phylum / phylogenetic } \\
\text { class }\end{array}$ & Species & $\begin{array}{c}\text { Accession } \\
\text { number }\end{array}$ & $\begin{array}{l}\text { isolation } \\
\text { medium }\end{array}$ & \multicolumn{2}{|c|}{ AM activity vs E.coli } & \multicolumn{2}{|c|}{ AM activity vs S.aureus } \\
\hline \multicolumn{4}{|l|}{ Proteobacteria } & Monoculture & Interaction & Monoculture & Interaction \\
\hline beta-proteobacteria & Janthinobacterium sp. AD $_{54}$ & KJ685267 & $\overline{T S B A}$ & & & $x$ & \\
\hline beta-proteobacteria & Janthinobacterium sp. AD 55 & KJ 685268 & TSBA & $\mathrm{x}$ & & & \\
\hline beta-proteobacteria & Collimonas sp. $\mathrm{AD}_{5} 8$ & KJ685270 & CHIT & & & & \\
\hline beta-proteobacteria & Collimonas sp. AD59 & KJ685271 & CHIT & & & & \\
\hline beta-proteobacteria & Collimonas sp. AD6o & $\mathrm{KJ} 685272$ & CHIT & & & & \\
\hline beta-proteobacteria & Collimonas sp. AD61 & KJ685273 & CHIT & & & & $x(1)$ \\
\hline beta-proteobacteria & Collimonas sp. AD62 & KJ685274 & CHIT & & & & $x(3)$ \\
\hline beta-proteobacteria & Collimonas sp. AD63 & KJ685275 & CHIT & & & $\mathrm{x}$ & $\mathrm{x}$ \\
\hline beta-proteobacteria & Collimonas sp. AD64 & KJ685276 & CHIT & & & $\mathrm{x}$ & \\
\hline beta-proteobacteria & Collimonas sp. AD65 & $\mathrm{KJ} 685277$ & CHIT & & & & $x(5)$ \\
\hline beta-proteobacteria & Collimonas sp. AD66 & $\mathrm{KJ} 685278$ & CHIT & & & $\mathbf{x}$ & \\
\hline beta-proteobacteria & Collimonas sp. AD67 & KJ685279 & CHIT & & & & $x(2)$ \\
\hline beta-proteobacteria & Collimonas sp. AD68 & KJ685280 & CHIT & & $x(1)$ & & $\mathbf{x}(\mathbf{1})$ \\
\hline beta-proteobacteria & Collimonas sp. AD69 & $\mathrm{KJ} 685281$ & CHIT & & & & $\mathrm{x}(3)$ \\
\hline beta-proteobacteria & Collimonas sp. AD7o & $\mathrm{KJ} 685282$ & CHIT & & & & $\mathbf{x}(\mathbf{1})$ \\
\hline beta-proteobacteria & Collimonas sp. $\mathrm{AD}_{71}$ & $\mathrm{KJ} 685283$ & CHIT & & & & $\mathbf{x}(\mathbf{1})$ \\
\hline beta-proteobacteria & Janthinobacterium sp. $\mathrm{AD}_{72}$ & $\begin{array}{l}\text { KJos } \\
\text { KJ685283 }\end{array}$ & CHIT & & $x(1)$ & & $\begin{array}{l}x(3) \\
\times(3)\end{array}$ \\
\hline beta-proteobacteria & Janthinobacterium sp. $\mathrm{AD}_{73}$ & $\mathrm{KJ} 685285$ & CHIT & & & & $x(1)$ \\
\hline beta-proteobacteria & Janthinobacterium sp. $\mathrm{AD}_{74}$ & KJ685286 & CHIT & $\mathrm{x}$ & & $\mathbf{x}$ & \\
\hline beta-proteobacteria & Janthinobacterium sp. $\mathrm{AD}_{75}$ & $\mathrm{KJ} 685287$ & CHIT & & & & $x(2)$ \\
\hline beta-proteobacteria & Collimonas sp. $\mathrm{AD}_{7} 6$ & KJ685288 & CHIT & & & $\mathbf{x}$ & $\mathrm{x}$ \\
\hline beta-proteobacteria & Collimonas sp. $\mathrm{AD}_{77}$ & KJ685289 & CHIT & $\mathrm{x}$ & & $\mathbf{x}$ & \\
\hline beta-proteobacteria & Janthinobacterium sp. AD8o & KJ685292 & CHIT & & $x(4)$ & $\mathrm{x}$ & \\
\hline beta-proteobacteria & Silvimonas sp. AD81 & KJ685293 & CHIT & & & $\mathrm{x}$ & \\
\hline beta-proteobacteria & Silvimonas sp. AD82 & KJ685294 & CHIT & & & $\mathrm{x}$ & \\
\hline beta-proteobacteria & Collimonas sp. AD88 & KJ685300 & CHIT & & & & $x(3)$ \\
\hline beta-proteobacteria & Collimonas sp. AD89 & KJ685301 & CHIT & & & & $\mathbf{x}(3)$ \\
\hline beta-proteobacteria & Collimonas sp. AD95 & KJ685306 & CHIT & $\mathrm{x}$ & & $\mathrm{x}$ & \\
\hline beta-proteobacteria & Janthinobacterium sp. AD96 & KJ685307 & CHIT & $\mathrm{x}$ & & $\mathrm{x}$ & \\
\hline beta-proteobacteria & Collimonas sp. AD97 & $\mathrm{KJ} 685308$ & CHIT & & & & $x(2)$ \\
\hline beta-proteobacteria & Collimonas sp. AD98 & KJ685309 & CHIT & & & & $\times(5)$ \\
\hline beta-proteobacteria & Collimonas sp. AD99 & KJ685310 & CHIT & & & & $\begin{array}{l}x(1) \\
x(1)\end{array}$ \\
\hline beta-proteobacteria & Collimonas sp. AD1o1 & KJ685312 & CHIT & & & & $\mathbf{x}(\mathbf{1})$ \\
\hline beta-proteobacteria & Collimonas sp. AD1o2 & $\mathrm{KJ} 685313$ & CHIT & & & & $x(1)$ \\
\hline beta-proteobacteria & Collimonas sp. AD1o3 & $\mathrm{KJ} 685314$ & CHIT & & & $\mathbf{x}$ & \\
\hline beta-proteobacteria & Janthinobacterium sp. ADı18 & $\mathrm{KJ} 685327$ & TSBA & $\mathbf{x}$ & & $\mathrm{x}$ & $\mathbf{x}$ \\
\hline beta-proteobacteria & Janthinobacterium sp. AD119 & KJ685328 & TSBA & $\mathrm{x}$ & & $\mathbf{x}$ & \\
\hline beta-proteobacteria & Burkholderia sp. AD123 & KJ685331 & TSBA & & & & \\
\hline beta-proteobacteria & Burkholderia sp. AD127 & KJ685335 & TSBA & $\mathrm{x}$ & & & \\
\hline beta-proteobacteria & $\begin{array}{l}\text { Variovorax sp. ADizo } \\
\text { ADis }\end{array}$ & KJ685337 & TSBA & & & & \\
\hline beta-proteobacteria & Variovorax sp. ADi33 & KJ685340 & TSBA & & $x(1)$ & $\mathbf{x}$ & \\
\hline beta-proteobacteria & Collimonas sp. AD137 & KJ685343 & TSBA & & & & $x(\mathbf{1})$ \\
\hline beta-proteobacteria & Burkholderia sp. ADi38 & KJ685344 & TSBA & & & & $\mathbf{x}(\mathbf{1})$ \\
\hline beta-proteobacteria & Variovorax sp. AD143 & KJ685348 & TSBA & & & & $x(5)$ \\
\hline beta-proteobacteria & Janthinobacterium sp. AD144 & KJ685349 & CHIT & & & $\mathbf{x}$ & \\
\hline beta-proteobacteria & Roseateles sp. AD145 & KJ685350 & CHIT & & & & $x(1)$ \\
\hline gamma-proteobacteria & Pseudomonas sp. AD2 & KJ685219 & TSBA & & & & \\
\hline gamma-proteobacteria & Pseudomonas sp. $\mathrm{AD}_{4}$ & KJ685221 & TSBA & & & $\mathrm{x}$ & \\
\hline gamma-proteobacteria & Pseudomonas sp. AD 5 & $\mathrm{KJ} 685222$ & TSBA & & & $\mathrm{x}$ & \\
\hline gamma-proteobacteria & Pseudomonas sp. AD6 & $\mathrm{KJ} 685223$ & TSBA & & & $\mathrm{x}$ & \\
\hline gamma-proteobacteria & Pseudomonas sp. $\mathrm{AD}_{7}$ & KJ685224 & TSBA & & & $\mathrm{x}$ & $\mathbf{x}$ \\
\hline gamma-proteobacteria & Pseudomonas sp. AD8 & $\mathrm{KJ} 685225$ & TSBA & & & $\mathrm{x}$ & \\
\hline gamma-proteobacteria & Pseudomonas sp. $\mathrm{AD}_{14}$ & KJ685230 & TSBA & & & $\mathrm{x}$ & \\
\hline gamma-proteobacteria & Pseudomonas sp. AD16 & $\mathrm{KJ} 685232$ & TSBA & & & $\mathrm{x}$ & \\
\hline gamma-proteobacteria & Pseudomonas sp. AD17 & KJ685233 & TSBA & & & $\mathrm{x}$ & \\
\hline gamma-proteobacteria & Pseudomonas sp. AD21 & $\mathrm{DQ}_{7780_{36}}$ & TSBA & $\mathrm{x}$ & & $\mathrm{x}$ & $\mathrm{x}$ \\
\hline gamma-proteobacteria & Pseudomonas sp. AD 36 & KJ685251 & TSBA & & & & \\
\hline gamma-proteobacteria & Pseudomonas sp. AD 79 & KJ685291 & CHIT & & & $\mathbf{x}$ & \\
\hline gamma-proteobacteria & Pseudomonas sp. ADioo & KJ685311 & CHIT & & & $\mathrm{x}$ & $\mathrm{x}$ \\
\hline gamma-proteobacteria & Pseudomonas sp. AD104 & KJ685315 & CHIT & & & & $x(5)$ \\
\hline gamma-proteobacteria & Pseudomonas sp. ADio5 & KJ685316 & CHIT & & & & $\begin{array}{l}x(2) \\
x(2)\end{array}$ \\
\hline gamma-proteobacteria & Pseudomonas sp. ADi14 & KJ685324 & TSBA & & $x(1)$ & & $x(1)$ \\
\hline gamma-proteobacteria & Pseudomonas sp. AD122 & $\mathrm{KJ} 685330$ & TSBA & & & & \\
\hline gamma-proteobacteria & Pseudomonas sp. AD124 & KJ685332 & TSBA & & & & $x(1)$ \\
\hline gamma-proteobacteria & Pseudomonas sp. AD125 & $\mathrm{KJ} 685333$ & TSBA & $\mathbf{x}$ & & & \\
\hline gamma-proteobacteria & Pseudomonas sp. AD157 & KJ685360 & TSBA & & & & $x(1)$ \\
\hline gamma-proteobacteria & Luteibactor sp. AD20 & KJ685236 & TSBA & & & $\mathbf{x}$ & \\
\hline gamma-proteobacteria & Dyella sp. $\mathrm{AD}_{4} \mathrm{o}$ & KJ685255 & TSBA & & & $\mathrm{x}$ & \\
\hline gamma-proteobacteria & Dyella sp. AD 46 & $\mathrm{KJ} 685261$ & TSBA & & & & $x(1)$ \\
\hline gamma-proteobacteria & Lysobacter sp. $\mathrm{AD}_{52}$ & $\mathrm{KJ} 685266$ & CHIT & & & $\mathrm{x}$ & \\
\hline gamma-proteobacteria & Dyella sp. AD 56 & KJ 685269 & TSBA & & $x(1)$ & $\hat{x}$ & \\
\hline gamma-proteobacteria & Frateuria sp. AD12o & KJ685329 & TSBA & & & & \\
\hline gamma-proteobacteria & Dyella sp. AD129 & $\mathrm{KJ} 685336$ & TSBA & & & & \\
\hline gamma-proteobacteria & Stenotrophomonas sp. AD147 & KJ685352 & CHIT & & & & $x(1)$ \\
\hline gamma-proteobacteria & Rhodonobacter sp. AD1o9 & KJ685320 & TSBA & & & & \\
\hline
\end{tabular}

\section{Abbreviations:}

AM activity $=$ antimicrobial activity against E.coli $\mathrm{WA}_{321}$ or S.aureus $533 \mathrm{R}_{4}$ in monoculture or in interactions. Isolation media: CHIT = chitin supplemented agar plates, TSBA $=$ Tryptic soy broth agar plates. Numbers in brackets represent the number of interactions that triggered antimicrobial activity either against E.coli WA321 or S.aureus 533R4. 


\section{Impact of interspecific interactions on antimicrobial activity among soil bacteria}

Table S2.2: Bacterial strains used as target and positive control in the highthrough-put interaction assay and the agar-overlay assay to detect antimicrobial activity.

\begin{tabular}{lcl}
\hline \multicolumn{1}{c}{ Organism } & Strain number & \multicolumn{1}{c}{ Assay function } \\
\hline Streptomyces rimosus & $\begin{array}{l}\text { DSMZ 40 260 } \\
\text { (type strain) } \\
\text { DSMZ 40 109 } \\
\text { (type strain) } \\
\text { DSMZ 40 500 } \\
\text { (type strain) }\end{array}$ & $\begin{array}{l}\text { positive control, produces } \\
\text { oxytetracycline } \\
\text { positive control, produces amphotericin } \\
\text { A and amphotericin B } \\
\text { positive control, produces kanamycin } \\
\text { A,B and C }\end{array}$ \\
$\begin{array}{lll}\text { Streptomyces kanamyceticus } \\
\text { Escherichia coli WA321 }\end{array}$ & DSMZ 4509 & target, model for human pathogen \\
$\begin{array}{l}\text { Staphylococcus aureus } 533 \mathrm{R}_{4} \\
\text { Serovar } 3\end{array}$ & DSMZ 20231 & target, model for human pathogen \\
\hline
\end{tabular}

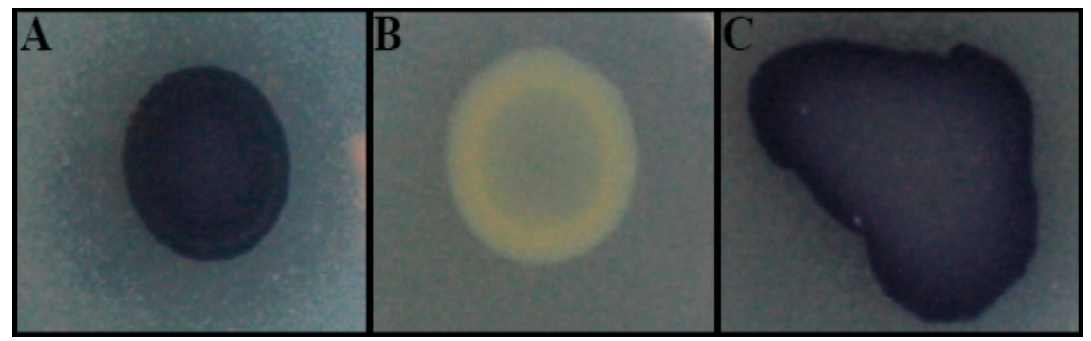

Figure S2.3: Suppression of antimicrobial activity revealed via the agar overlay assay: Janthinobacterium sp. AD55 monoculture (A), Flavobacterium sp. AD86 monoculture (B), Interaction Janthinobacterium sp. AD55 with Flavobacterium sp. AD86 (C) loss of antimicrobial activity against E. coli WA321.

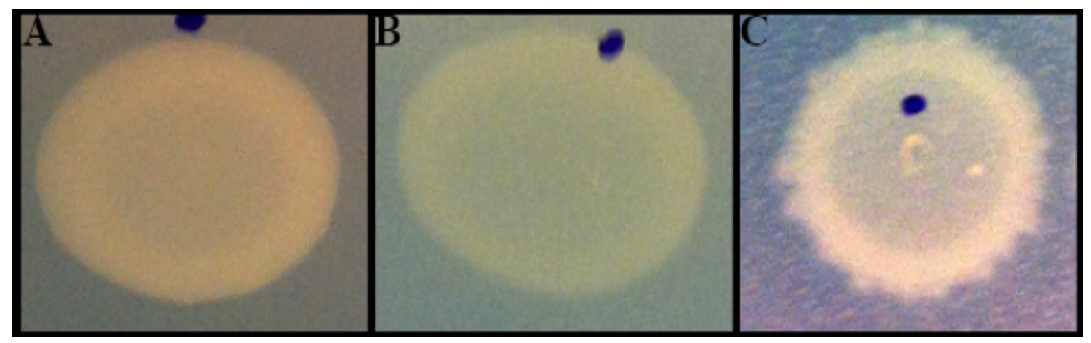

Figure S2.4: Induction of antimicrobial activity revealed via the agar overlay assay: (A) Dyella sp. AD56 monoculture, (B) Janthinobacterium sp. AD8o monoculture (C) interaction Dyella sp. AD56 with Janthinobacterium sp. AD8o, antimicrobial activity against $E$. coli WA321. 
Table S2.5: Results of the Chi-square test for frequencies of induction of antimicrobial activity against (A) E.coli WA321 and (B) S.aureus 533R4.

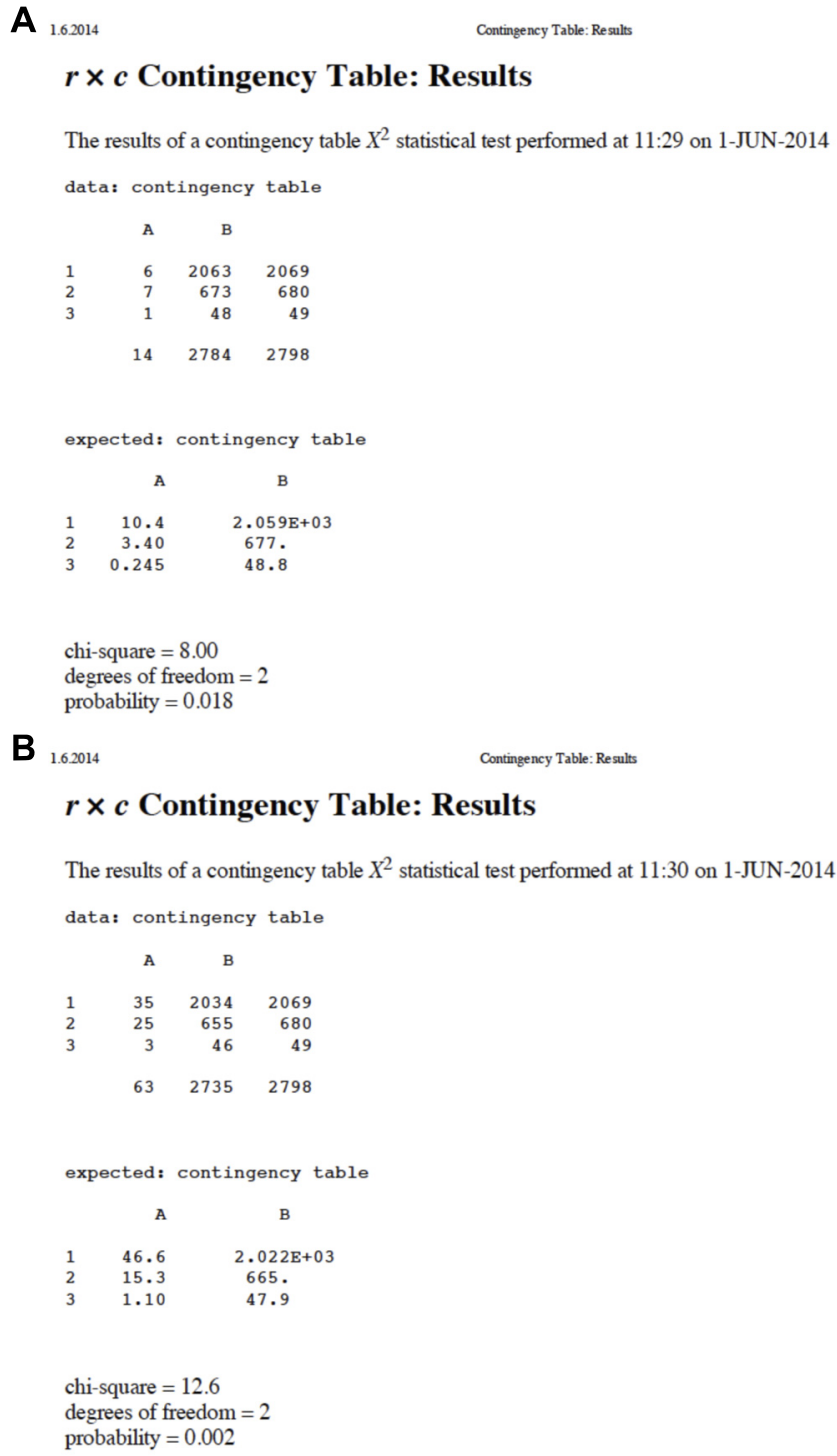

B 1.62014

Contingency Table: Re sults

\section{$r \times c$ Contingency Table: Results}

Abbreviations: Rows: Gram-group interactions (1: Gram-/Gram- interactions, 2: Gram+/Gram- interactions, 3: Gram + /Gram+ interactions). Columns: Number of interactions with A: triggering of antimicrobial activity and $\mathbf{B}$ : Interactions without triggering. 


\section{Impact of interspecific interactions on antimicrobial activity among soil bacteria}

Table S2.6: Results of the Chi-square test for the frequencies of silencing of antimicrobial activity against (A) E.coli WA 321 and (B) S.aureus 533R4.

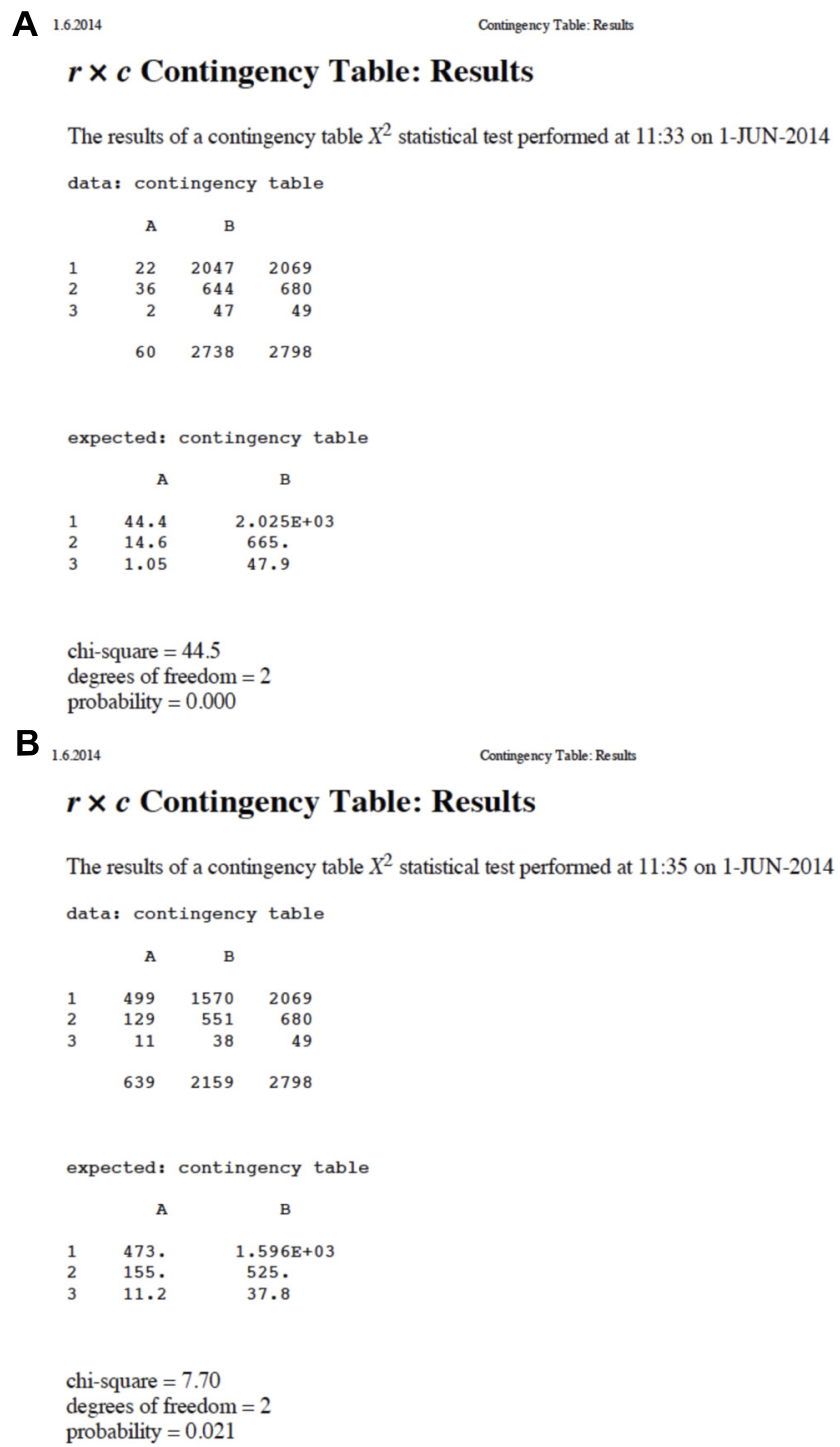

Abbreviations: Rows: Gram-group interactions (1: Gram-/Gram- interactions, 2: Gram+/Gram- interactions, 3: Gram+/Gram+ interactions). Columns: Number of interactions with A: observed silencing of antimicrobial activity and $\mathbf{B}$ : without observed silencing. 
Table S2.7: Bacterial isolates without any antimicrobial activity against E.coli WA 321 or S.aureus $533 \mathrm{R}_{4}$ either in paired combination or monoculture.

\begin{tabular}{|c|c|c|c|c|c|c|c|}
\hline $\begin{array}{c}\text { Phylum / } \\
\text { phylogenetic class }\end{array}$ & Species & $\begin{array}{c}\text { Accession } \\
\text { number }\end{array}$ & $\begin{array}{l}\text { isolation } \\
\text { medium }\end{array}$ & \multicolumn{2}{|c|}{ AM activity vs E.coli } & \multicolumn{2}{|c|}{ AM activity vs S.aureus } \\
\hline Bacteroidetes & & & & Monoculture & Interaction & Monoculture & Interaction \\
\hline Flavobacteria & Flavobacterium sp. AD131 & KJ685338 & TSBA & - & - & - & - \\
\hline Flavobacteria & Flavobacterium sp. AD134 & KJ685341 & TSBA & - & - & - & - \\
\hline Flavobacteria & Flavobacterium sp. AD149 & KJ685354 & TSBA & - & - & - & - \\
\hline \multicolumn{8}{|l|}{ Proteobacteria } \\
\hline alpha-proteobacteria & Agrobacterium sp. AD1 & KJ685218 & TSBA & - & - & - & - \\
\hline alpha-proteobacteria & Bradyrhizobiaceae sp. ADı26 & KJ685334 & TSBA & - & - & - & - \\
\hline beta-proteobacteria & Burkholderia sp. $\mathrm{AD}_{3}$ & KJ685220 & TSBA & - & - & - & - \\
\hline beta-proteobacteria & Collimonas sp. AD58 & KJ68527o & CHIT & - & - & - & - \\
\hline beta-proteobacteria & Collimonas sp. $\mathrm{AD}_{59}$ & KJ685271 & CHIT & - & - & - & - \\
\hline beta-proteobacteria & Collimonas sp. AD6o & KJ685272 & CHIT & - & - & - & - \\
\hline beta-proteobacteria & Burkholderia sp. AD123 & KJ685331 & TSBA & - & - & - & - \\
\hline beta-proteobacteria & Variovorax sp. ADizo & KJ685337 & TSBA & - & - & - & - \\
\hline gamma-proteobacteria & Pseudomonas sp. $\mathrm{AD}_{2}$ & KJ685219 & TSBA & - & - & - & - \\
\hline gamma-proteobacteria & Pseudomonas sp. $\mathrm{AD}_{36}$ & KJ685251 & TSBA & - & - & - & - \\
\hline gamma-proteobacteria & Rhodonobacter sp. ADiog & KJ685320 & TSBA & - & - & - & - \\
\hline gamma-proteobacteria & Frateuria sp. AD12o & KJ685329 & TSBA & - & - & - & - \\
\hline gamma-proteobacteria & Pseudomonas sp. AD122 & KJ685330 & TSBA & - & - & - & - \\
\hline gamma-proteobacteria & Dyella sp. AD129 & KJ685336 & TSBA & - & - & - & - \\
\hline
\end{tabular}




\section{Impact of interspecific interactions on antimicrobial activity among soil bacteria}

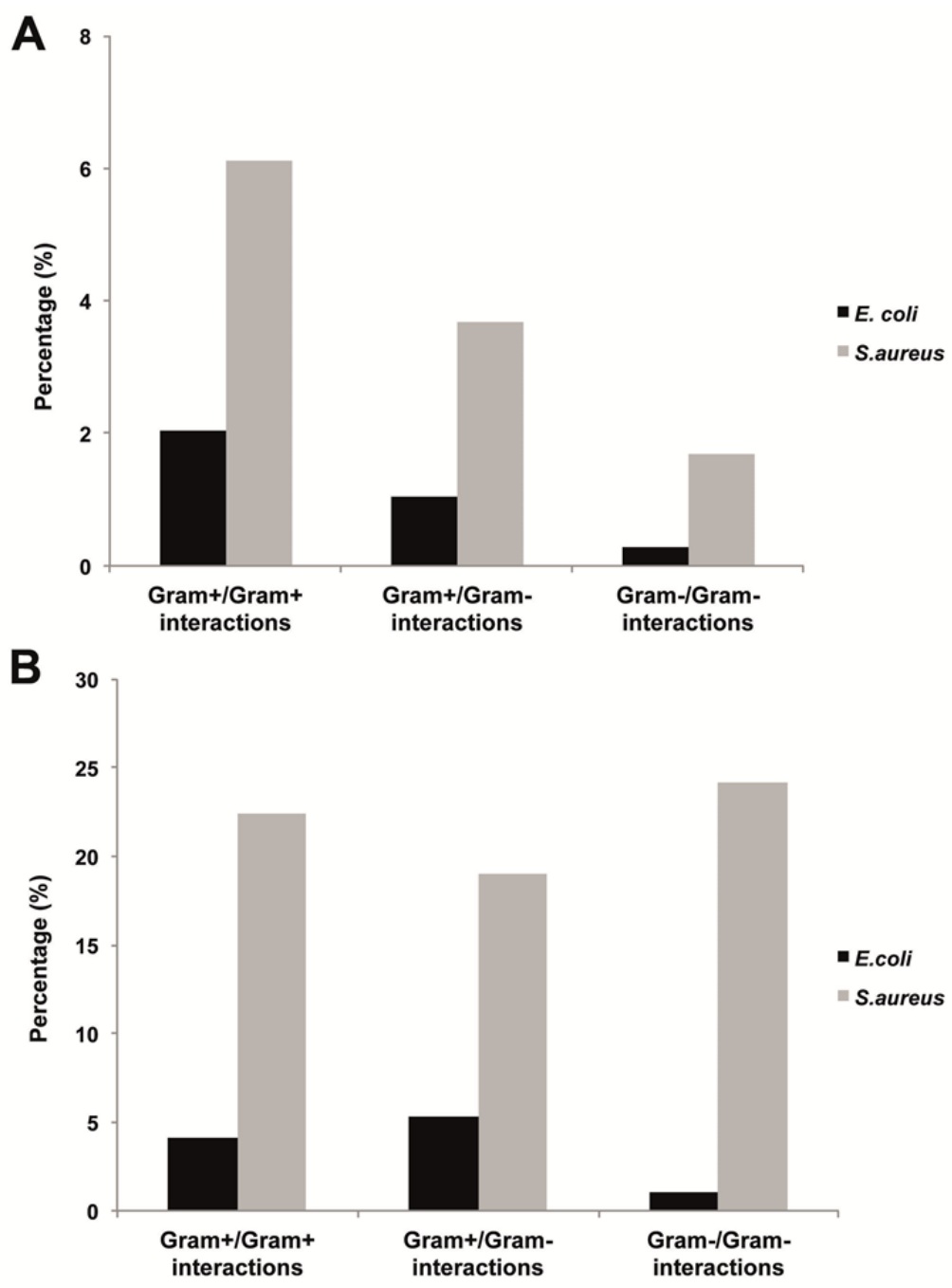

Figure S2.8: Frequencies of induction (A) or suppression of (B) antimicrobial activities for combinations of Gram- or Gram+ bacterial isolates. Antimicrobial activities were tested against E.coli WA321 or S.aureus $533 \mathrm{R} 4$. Number of tested interactions: Gram+/Gram+: $n=49$, Gram+/Gram-: $n=690$, Gram-/Gram-: $n=2069$. The results of the statistical analyses (Chi-square tests) are presented in Table $\mathbf{S 2 . 5}$ and $\mathbf{S 2 . 6 .}$ 
Table S2.9: Bacterial combination pairs which suppressed antimicrobial activity against E.coli WA 321. For the strain designation please see Table S2.1.

\begin{tabular}{|c|c|c|c|}
\hline Phylogenetic class & Strain code genus A & Phylogenetic class & Strain code genus B \\
\hline Bacilli & AD50 & beta-proteobacteria & AD10 \\
\hline Bacilli & AD5o & Actinobacteria & AD22 \\
\hline Bacilli & AD5o & alpha-proteobacteria & $\mathrm{AD}_{34}$ \\
\hline Bacilli & $\mathrm{AD}_{5} \mathrm{O}$ & beta-proteobacteria & AD6o \\
\hline Bacilli & AD50 & beta-proteobacteria & $A D_{70}$ \\
\hline Bacilli & AD5o & beta-proteobacteria & AD9 \\
\hline Bacilli & $\mathrm{AD}_{5} \mathrm{O}$ & beta-proteobacteria & AD26 \\
\hline Bacilli & AD50 & beta-proteobacteria & AD81 \\
\hline Bacilli & AD50 & beta-proteobacteria & AD98 \\
\hline Bacilli & $\mathrm{AD}_{50}$ & beta-proteobacteria & AD89 \\
\hline Bacilli & $\mathrm{AD}_{5} \mathrm{O}$ & Bacilli & AD87 \\
\hline Bacilli & $\mathrm{AD}_{50}$ & beta-proteobacteria & AD137 \\
\hline Bacilli & AD5o & alpha-proteobacteria & ADi48 \\
\hline Bacilli & AD5o & gamma-proteobacteria & AD157 \\
\hline Bacilli & $\mathrm{AD}_{5} \mathrm{O}$ & Flavobacteria & AD155 \\
\hline Bacilli & AD 50 & beta-proteobacteria & AD25 \\
\hline Bacilli & $\mathrm{AD}_{5} \mathrm{O}$ & beta-proteobacteria & $\mathrm{AD}_{33}$ \\
\hline Bacilli & AD50 & Bacilli & AD83 \\
\hline Bacilli & AD50 & beta-proteobacteria & ADior \\
\hline Bacilli & AD50 & gamma-proteobacteria & AD125 \\
\hline Bacilli & $\mathrm{AD}_{5} \mathrm{O}$ & Flavobacteria & AD142 \\
\hline Bacilli & ADi17 & gamma-proteobacteria & AD2o \\
\hline Bacilli & AD117 & Flavobacteria & $\mathrm{AD}_{42}$ \\
\hline Bacilli & ADi17 & beta-proteobacteria & AD68 \\
\hline Bacilli & ADi17 & beta-proteobacteria & $\mathrm{AD}_{24}$ \\
\hline Bacilli & AD117 & beta-proteobacteria & AD95 \\
\hline Bacilli & AD117 & Flavobacteria & AD146 \\
\hline Bacilli & AD117 & alpha-proteobacteria & AD159 \\
\hline Bacilli & AD117 & gamma-proteobacteria & AD14 \\
\hline Bacilli & AD117 & gamma-proteobacteria & AD56 \\
\hline Bacilli & AD117 & beta-proteobacteria & AD69 \\
\hline Bacilli & ADi17 & beta-proteobacteria & AD98 \\
\hline Bacilli & AD117 & alpha-proteobacteria & ADoor \\
\hline Bacilli & ADi17 & Actinobacteria & AD12 \\
\hline Bacilli & AD117 & gamma-proteobacteria & $\mathrm{AD} 2$ \\
\hline beta-proteobacteria & AD 55 & beta-proteobacteria & ADı \\
\hline beta-proteobacteria & AD 55 & beta-proteobacteria & $\mathrm{AD}_{23}$ \\
\hline beta-proteobacteria & AD 55 & beta-proteobacteria & $\mathrm{AD}_{35}$ \\
\hline beta-proteobacteria & $\mathrm{AD}_{55}$ & Flavobacteria & $\mathrm{AD}_{45}$ \\
\hline beta-proteobacteria & $\mathrm{AD}_{55}$ & beta-proteobacteria & AD61 \\
\hline beta-proteobacteria & AD 55 & beta-proteobacteria & $A D_{71}$ \\
\hline beta-proteobacteria & AD 55 & beta-proteobacteria & AD15 \\
\hline beta-proteobacteria & $\mathrm{AD}_{55}$ & Actinobacteria & AD22 \\
\hline beta-proteobacteria & $\mathrm{AD}_{55}$ & beta-proteobacteria & AD82 \\
\hline beta-proteobacteria & AD 55 & gamma-proteobacteria & ADioo \\
\hline beta-proteobacteria & $\mathrm{AD}_{55}$ & beta-proteobacteria & AD98 \\
\hline beta-proteobacteria & $\mathrm{AD}_{55}$ & beta-proteobacteria & $\mathrm{AD}_{138} 8$ \\
\hline beta-proteobacteria & AD 55 & beta-proteobacteria & ADi43 \\
\hline beta-proteobacteria & AD 55 & gamma-proteobacteria & AD147 \\
\hline beta-proteobacteria & AD 55 & beta-proteobacteria & ADıo \\
\hline beta-proteobacteria & AD 55 & beta-proteobacteria & AD6o \\
\hline beta-proteobacteria & AD 55 & Flavobacteria & AD86 \\
\hline beta-proteobacteria & $\mathrm{AD}_{55}$ & beta-proteobacteria & AD102 \\
\hline beta-proteobacteria & AD 55 & beta-proteobacteria & $\mathrm{AD}_{74}$ \\
\hline beta-proteobacteria & AD 55 & alpha-proteobacteria & AD112 \\
\hline beta-proteobacteria & AD 55 & alpha-proteobacteria & AD126 \\
\hline gamma-proteobacteria & AD125 & beta-proteobacteria & AD66 \\
\hline gamma-proteobacteria & AD125 & Bacilli & AD5o \\
\hline beta-proteobacteria & AD127 & beta-proteobacteria & $\mathrm{AD}_{75}$ \\
\hline beta-proteobacteria & AD127 & Actinobacteria & AD107 \\
\hline
\end{tabular}




\section{Impact of interspecific interactions on antimicrobial activity among soil bacteria}

Table S2.10: Bacterial combination pairs which suppressed antimicrobial activity against S.aureus $533 \mathrm{R}_{4}$. For the strain designation please see Table

S2.1.

\begin{tabular}{|c|c|c|c|}
\hline Phylogenetic class & Strain code genus A & Phylogenetic class & Strain code genus B \\
\hline Actinobacteria & AD107 & gamma-proteobacteria & $\mathrm{AD} 14$ \\
\hline Actinobacteria & ADio7 & beta-proteobacteria & $\mathrm{AD}_{24}$ \\
\hline Actinobacteria & ADio7 & gamma-proteobacteria & $\mathrm{AD}_{3} 6$ \\
\hline Actinobacteria & AD107 & beta-proteobacteria & AD62 \\
\hline Actinobacteria & ADio7 & beta-proteobacteria & $\mathrm{AD}_{72}$ \\
\hline Actinobacteria & ADio7 & beta-proteobacteria & $\mathrm{AD}_{32}$ \\
\hline Actinobacteria & ADio7 & Bacilli & $\mathrm{AD}_{3}$ \\
\hline Actinobacteria & AD107 & beta-proteobacteria & AD99 \\
\hline Actinobacteria & ADio7 & gamma-proteobacteria & ADioo \\
\hline Actinobacteria & AD107 & alpha-proteobacteria & AD140 \\
\hline Actinobacteria & $\mathrm{AD}_{10} 7$ & Actinobacteria & AD22 \\
\hline Actinobacteria & ADio7 & Bacilli & $\mathrm{ADB}_{7}$ \\
\hline Actinobacteria & AD107 & beta-proteobacteria & ADio3 \\
\hline Actinobacteria & ADio7 & beta-proteobacteria & AD127 \\
\hline Actinobacteria & AD107 & Flavobacteria & AD146 \\
\hline Flavobacteria & $\mathrm{AD}_{41}$ & beta-proteobacteria & AD19 \\
\hline Flavobacteria & $\mathrm{AD}_{41}$ & beta-proteobacteria & $\mathrm{AD6}_{7}$ \\
\hline Flavobacteria & $\mathrm{AD}_{41}$ & beta-proteobacteria & $\mathrm{AD}_{25}$ \\
\hline Flavobacteria & $\mathrm{AD}_{41}$ & Actinobacteria & AD94 \\
\hline Flavobacteria & $\mathrm{AD}_{41}$ & beta-proteobacteria & AD96 \\
\hline Flavobacteria & $\mathrm{AD}_{41}$ & beta-proteobacteria & AD145 \\
\hline Flavobacteria & $\mathrm{AD}_{41}$ & Flavobacteria & AD151 \\
\hline Flavobacteria & $\mathrm{AD}_{41}$ & gamma-proteobacteria & AD16 \\
\hline Flavobacteria & $\mathrm{AD}_{41}$ & beta-proteobacteria & $\mathrm{AD}_{54}$ \\
\hline Flavobacteria & $\mathrm{AD}_{41}$ & gamma-proteobacteria & AD8 \\
\hline Flavobacteria & $\mathrm{AD}_{41}$ & beta-proteobacteria & AD68 \\
\hline Flavobacteria & $\mathrm{AD}_{41}$ & beta-proteobacteria & $\mathrm{AD}_{32}$ \\
\hline Flavobacteria & $\mathrm{AD}_{41}$ & beta-proteobacteria & $\mathrm{AD}_{5} 8$ \\
\hline Flavobacteria & $\mathrm{AD}_{41}$ & gamma-proteobacteria & $\mathrm{AD}_{5}$ \\
\hline Flavobacteria & $\mathrm{AD}_{41}$ & gamma-proteobacteria & $\mathrm{AD}_{79}$ \\
\hline Flavobacteria & $\mathrm{AD}_{41}$ & Bacilli & $\mathrm{AD}_{3}$ \\
\hline Flavobacteria & $\mathrm{AD}_{41}$ & Flavobacteria & AD134 \\
\hline Flavobacteria & $\mathrm{AD}_{41}$ & Flavobacteria & ADi42 \\
\hline Flavobacteria & $\mathrm{AD}_{41}$ & beta-proteobacteria & $\mathrm{AD}_{24}$ \\
\hline Flavobacteria & $\mathrm{AD}_{41}$ & Bacilli & AD93 \\
\hline Flavobacteria & $\mathrm{AD}_{41}$ & beta-proteobacteria & $\mathrm{AD}_{27}$ \\
\hline Bacilli & $\mathrm{AD8}_{7}$ & gamma-proteobacteria & AD16 \\
\hline Bacilli & AD87 & Actinobacteria & $\mathrm{AD}_{22}$ \\
\hline Bacilli & AD87 & alpha-proteobacteria & $\mathrm{AD}_{3} 8$ \\
\hline Bacilli & $\mathrm{ADB}_{7}$ & Flavobacteria & $\mathrm{AD}_{44}$ \\
\hline Bacilli & AD87 & beta-proteobacteria & AD64 \\
\hline Bacilli & AD87 & beta-proteobacteria & $A D_{7} \mathrm{o}$ \\
\hline Bacilli & AD87 & gamma-proteobacteria & $\mathrm{AD}_{2} \mathrm{O}$ \\
\hline Bacilli & AD87 & beta-proteobacteria & $\mathrm{AD}_{2} 6$ \\
\hline Bacilli & $\mathrm{ADB}_{7}$ & beta-proteobacteria & AD98 \\
\hline Bacilli & $\mathrm{AD8}_{7}$ & Bacilli & ADio6 \\
\hline Bacilli & AD87 & Flavobacteria & $\mathrm{AD}_{142}$ \\
\hline Bacilli & AD87 & Flavobacteria & ADi49 \\
\hline Bacilli & AD87 & gamma-proteobacteria & $\mathrm{AD}_{21}$ \\
\hline Bacilli & $\mathrm{AD8}_{7}$ & Flavobacteria & $\mathrm{AD}_{4} 8$ \\
\hline Bacilli & $\mathrm{ADB}_{7}$ & alpha-proteobacteria & ADi48 \\
\hline Bacilli & AD87 & Flavobacteria & AD155 \\
\hline Bacilli & AD87 & beta-proteobacteria & AD1o \\
\hline Bacilli & AD87 & beta-proteobacteria & $\mathrm{AD}_{95}$ \\
\hline Bacilli & $\mathrm{AD8}_{7}$ & beta-proteobacteria & AD65 \\
\hline Bacilli & $\mathrm{ADB}_{7}$ & beta-proteobacteria & $\mathrm{AD}_{71}$ \\
\hline Bacilli & AD87 & gamma-proteobacteria & $\mathrm{AD}_{14}$ \\
\hline Bacilli & AD87 & gamma-proteobacteria & $\mathrm{AD}_{3} 6$ \\
\hline Bacilli & AD87 & beta-proteobacteria & AD62 \\
\hline Bacilli & $\mathrm{AD8}_{7}$ & beta-proteobacteria & AD89 \\
\hline Bacilli & AD87 & Bacilli & $\mathrm{AD}_{3}$ \\
\hline Bacilli & $\mathrm{AD} 8_{7}$ & beta-proteobacteria & AD1o1 \\
\hline
\end{tabular}


Chapter Two

Table S2.10 continuation

\begin{tabular}{|c|c|c|c|}
\hline Phylogenetic class & Strain code genus A & Phylogenetic class & Strain code genus $B$ \\
\hline Bacilli & AD87 & gamma-proteobacteria & AD10o \\
\hline Bacilli & AD8 7 & alpha-proteobacteria & AD140 \\
\hline Bacilli & AD87 & beta-proteobacteria & AD144 \\
\hline Bacilli & AD87 & Actinobacteria & ADno \\
\hline Bacilli & AD8 7 & Bacilli & AD5o \\
\hline Bacilli & AD87 & Actinobacteria & AD107 \\
\hline Bacilli & AD93 & beta-proteobacteria & AD18 \\
\hline Bacilli & AD93 & gamma-proteobacteria & $\mathrm{AD}_{40}$ \\
\hline Bacilli & AD93 & beta-proteobacteria & AD66 \\
\hline Bacilli & AD93 & beta-proteobacteria & AD19 \\
\hline Bacilli & AD93 & gamma-proteobacteria & ADn14 \\
\hline Bacilli & AD93 & beta-proteobacteria & AD144 \\
\hline Bacilli & AD93 & alpha-proteobacteria & AD152 \\
\hline Bacilli & AD93 & gamma-proteobacteria & AD17 \\
\hline Bacilli & AD93 & gamma-proteobacteria & $\mathrm{AD}_{52}$ \\
\hline Bacilli & AD93 & beta-proteobacteria & AD67 \\
\hline Bacilli & AD93 & Flavobacteria & $\mathrm{AD}_{41}$ \\
\hline Bacilli & AD93 & beta-proteobacteria & $\mathrm{AD}_{25}$ \\
\hline Bacilli & AD93 & Actinobacteria & AD92 \\
\hline Bacilli & AD93 & Actinobacteria & AD94 \\
\hline Bacilli & AD93 & beta-proteobacteria & AD96 \\
\hline Bacilli & AD93 & beta-proteobacteria & AD145 \\
\hline Bacilli & AD93 & Flavobacteria & AD151 \\
\hline Bacilli & AD93 & gamma-proteobacteria & AD16 \\
\hline Bacilli & AD93 & beta-proteobacteria & AD119 \\
\hline Bacilli & AD93 & beta-proteobacteria & $\mathrm{AD}_{7}$ \\
\hline alpha-proteobacteria & AD113 & gamma-proteobacteria & AD17 \\
\hline alpha-proteobacteria & AD113 & beta-proteobacteria & $\mathrm{AD}_{39}$ \\
\hline alpha-proteobacteria & AD113 & beta-proteobacteria & AD65 \\
\hline alpha-proteobacteria & ADn13 & gamma-proteobacteria & $\mathrm{AD}_{21}$ \\
\hline alpha-proteobacteria & ADn13 & Actinobacteria & AD92 \\
\hline alpha-proteobacteria & ADn13 & beta-proteobacteria & AD143 \\
\hline alpha-proteobacteria & ADn13 & alpha-proteobacteria & AD153 \\
\hline alpha-proteobacteria & ADi13 & beta-proteobacteria & AD19 \\
\hline alpha-proteobacteria & AD113 & alpha-proteobacteria & $A D_{51}$ \\
\hline alpha-proteobacteria & AD113 & beta-proteobacteria & AD66 \\
\hline alpha-proteobacteria & AD113 & Flavobacteria & AD91 \\
\hline alpha-proteobacteria & AD113 & Flavobacteria & AD146 \\
\hline alpha-proteobacteria & AD113 & beta-proteobacteria & AD118 \\
\hline gamma-proteobacteria & $\mathrm{AD}_{4}$ & beta-proteobacteria & AD28 \\
\hline gamma-proteobacteria & $\mathrm{AD}_{4}$ & gamma-proteobacteria & $\mathrm{AD}_{52}$ \\
\hline gamma-proteobacteria & $\mathrm{AD}_{4}$ & gamma-proteobacteria & AD8 \\
\hline gamma-proteobacteria & $\mathrm{AD}_{4}$ & gamma-proteobacteria & $\mathrm{AD}_{5}$ \\
\hline gamma-proteobacteria & $\mathrm{AD}_{4}$ & beta-proteobacteria & $\mathrm{AD}_{76}$ \\
\hline gamma-proteobacteria & $\mathrm{AD}_{4}$ & gamma-proteobacteria & AD104 \\
\hline gamma-proteobacteria & $\mathrm{AD}_{4}$ & Flavobacteria & AD131 \\
\hline gamma-proteobacteria & $\mathrm{AD}_{4}$ & alpha-proteobacteria & AD140 \\
\hline gamma-proteobacteria & $\mathrm{AD}_{4}$ & beta-proteobacteria & $\mathrm{AD}_{30}$ \\
\hline gamma-proteobacteria & $\mathrm{AD}_{4}$ & beta-proteobacteria & AD26 \\
\hline gamma-proteobacteria & $\mathrm{AD}_{4}$ & Actinobacteria & AD29 \\
\hline gamma-proteobacteria & $\mathrm{AD}_{4}$ & alpha-proteobacteria & $\mathrm{AD}_{51}$ \\
\hline gamma-proteobacteria & $\mathrm{AD}_{4}$ & beta-proteobacteria & $\mathrm{AD}_{54}$ \\
\hline gamma-proteobacteria & $\mathrm{AD}_{4}$ & beta-proteobacteria & $\mathrm{AD}_{74}$ \\
\hline gamma-proteobacteria & $\mathrm{AD}_{4}$ & beta-proteobacteria & $\mathrm{AD}_{77}$ \\
\hline gamma-proteobacteria & $\mathrm{AD}_{4}$ & gamma-proteobacteria & AD105 \\
\hline gamma-proteobacteria & $\mathrm{AD}_{4}$ & alpha-proteobacteria & AD132 \\
\hline gamma-proteobacteria & $\mathrm{AD}_{4}$ & beta-proteobacteria & AD145 \\
\hline gamma-proteobacteria & $\mathrm{AD}_{4}$ & beta-proteobacteria & $\mathrm{AD}_{39}$ \\
\hline gamma-proteobacteria & $\mathrm{AD}_{4}$ & beta-proteobacteria & ADoog \\
\hline gamma-proteobacteria & $\mathrm{AD}_{4}$ & beta-proteobacteria & AD103 \\
\hline gamma-proteobacteria & $\mathrm{AD}_{4}$ & gamma-proteobacteria & AD120 \\
\hline
\end{tabular}




\section{Impact of interspecific interactions on antimicrobial activity among soil bacteria}

\begin{tabular}{|c|c|c|c|}
\hline Phylogenetic class & Strain code genus A & Phylogenetic class & Strain code genus B \\
\hline gamma-proteobacteria & $\mathrm{AD}_{4}$ & alpha-proteobacteria & AD153 \\
\hline gamma-proteobacteria & $\mathrm{AD}_{4}$ & beta-proteobacteria & AD137 \\
\hline gamma-proteobacteria & $\mathrm{AD}_{4}$ & beta-proteobacteria & AD64 \\
\hline gamma-proteobacteria & $\mathrm{AD}_{4}$ & alpha-proteobacteria & $\mathrm{AD}_{34}$ \\
\hline gamma-proteobacteria & $\mathrm{AD}_{4}$ & beta-proteobacteria & $\mathrm{AD}_{37}$ \\
\hline gamma-proteobacteria & $\mathrm{AD}_{4}$ & Bacilli & $\mathrm{AD}_{7} 8$ \\
\hline gamma-proteobacteria & $\mathrm{AD}_{4}$ & gamma-proteobacteria & $\mathrm{AD}_{2}$ \\
\hline gamma-proteobacteria & $\mathrm{AD}_{4}$ & beta-proteobacteria & $\mathrm{AD}_{3}$ \\
\hline gamma-proteobacteria & $\mathrm{AD}_{5}$ & Actinobacteria & AD29 \\
\hline gamma-proteobacteria & $\mathrm{AD}_{5}$ & beta-proteobacteria & $\mathrm{AD}_{54}$ \\
\hline gamma-proteobacteria & $\mathrm{AD}_{5}$ & gamma-proteobacteria & $\mathrm{AD}_{4}$ \\
\hline gamma-proteobacteria & $\mathrm{AD}_{5}$ & gamma-proteobacteria & AD8 \\
\hline gamma-proteobacteria & $\mathrm{AD}_{5}$ & beta-proteobacteria & $\mathrm{AD}_{77}$ \\
\hline gamma-proteobacteria & $\mathrm{AD}_{5}$ & gamma-proteobacteria & AD105 \\
\hline gamma-proteobacteria & $\mathrm{AD}_{5}$ & alpha-proteobacteria & AD132 \\
\hline gamma-proteobacteria & $\mathrm{AD}_{5}$ & beta-proteobacteria & AD145 \\
\hline gamma-proteobacteria & $\mathrm{AD}_{5}$ & beta-proteobacteria & $\mathrm{AD}_{25}$ \\
\hline gamma-proteobacteria & $\mathrm{AD}_{5}$ & beta-proteobacteria & ADoog \\
\hline gamma-proteobacteria & $\mathrm{AD}_{5}$ & beta-proteobacteria & $\mathrm{AD}_{32}$ \\
\hline gamma-proteobacteria & $\mathrm{AD}_{5}$ & Flavobacteria & $\mathrm{AD}_{4} 8$ \\
\hline gamma-proteobacteria & $\mathrm{AD}_{5}$ & beta-proteobacteria & $\mathrm{AD}_{5} 8$ \\
\hline gamma-proteobacteria & $\mathrm{AD}_{5}$ & beta-proteobacteria & $\mathrm{AD}_{73}$ \\
\hline gamma-proteobacteria & $\mathrm{AD}_{5}$ & gamma-proteobacteria & $\mathrm{AD}_{79}$ \\
\hline gamma-proteobacteria & $\mathrm{AD}_{5}$ & Bacilli & $\mathrm{AD8}_{3}$ \\
\hline gamma-proteobacteria & $\mathrm{AD}_{5}$ & Flavobacteria & AD134 \\
\hline gamma-proteobacteria & $\mathrm{AD}_{5}$ & Flavobacteria & AD142 \\
\hline gamma-proteobacteria & $\mathrm{AD}_{5}$ & beta-proteobacteria & $\mathrm{AD}_{37}$ \\
\hline gamma-proteobacteria & $\mathrm{AD}_{5}$ & beta-proteobacteria & $\mathrm{AD}_{27}$ \\
\hline gamma-proteobacteria & $\mathrm{AD}_{5}$ & beta-proteobacteria & $\mathrm{AD}_{24}$ \\
\hline gamma-proteobacteria & $\mathrm{AD}_{5}$ & beta-proteobacteria & AD102 \\
\hline gamma-proteobacteria & $\mathrm{AD}_{5}$ & Bacilli & AD116 \\
\hline gamma-proteobacteria & $\mathrm{AD}_{5}$ & alpha-proteobacteria & AD152 \\
\hline gamma-proteobacteria & $\mathrm{AD}_{5}$ & alpha-proteobacteria & AD136 \\
\hline gamma-proteobacteria & $\mathrm{AD}_{5}$ & beta-proteobacteria & $\mathrm{AD}_{3}$ \\
\hline gamma-proteobacteria & $\mathrm{AD}_{5}$ & Flavobacteria & $\mathrm{AD}_{41}$ \\
\hline gamma-proteobacteria & $\mathrm{AD}_{5}$ & Bacilli & $\mathrm{AD}_{7} 8$ \\
\hline gamma-proteobacteria & $\mathrm{AD}_{5}$ & beta-proteobacteria & AD81 \\
\hline gamma-proteobacteria & $\mathrm{AD}_{5}$ & gamma-proteobacteria & AD104 \\
\hline gamma-proteobacteria & $\mathrm{AD}_{5}$ & beta-proteobacteria & $\mathrm{AD}_{75}$ \\
\hline gamma-proteobacteria & $\mathrm{AD}_{5}$ & beta-proteobacteria & $\mathrm{AD}_{3}$ \\
\hline gamma-proteobacteria & $\mathrm{AD}_{5}$ & Actinobacteria & AD12 \\
\hline gamma-proteobacteria & $\mathrm{AD}_{5}$ & Actinobacteria & AD108 \\
\hline gamma-proteobacteria & AD6 & beta-proteobacteria & $\mathrm{AD}_{30}$ \\
\hline gamma-proteobacteria & AD6 & gamma-proteobacteria & $\mathrm{AD}_{5} 6$ \\
\hline gamma-proteobacteria & AD6 & Bacilli & $\mathrm{AD}_{7} 8$ \\
\hline gamma-proteobacteria & AD6 & beta-proteobacteria & AD81 \\
\hline gamma-proteobacteria & AD6 & beta-proteobacteria & AD133 \\
\hline gamma-proteobacteria & AD6 & Actinobacteria & AD141 \\
\hline gamma-proteobacteria & AD6 & beta-proteobacteria & $\mathrm{AD}_{28}$ \\
\hline gamma-proteobacteria & AD6 & beta-proteobacteria & $\mathrm{AD}_{24}$ \\
\hline gamma-proteobacteria & AD6 & beta-proteobacteria & AD11 \\
\hline gamma-proteobacteria & AD6 & beta-proteobacteria & $\mathrm{AD}_{30}$ \\
\hline gamma-proteobacteria & AD6 & gamma-proteobacteria & $\mathrm{AD}_{46}$ \\
\hline gamma-proteobacteria & AD6 & beta-proteobacteria & $\mathrm{AD}_{72}$ \\
\hline gamma-proteobacteria & AD6 & beta-proteobacteria & $\mathrm{AD}_{32}$ \\
\hline gamma-proteobacteria & AD6 & beta-proteobacteria & AD99 \\
\hline gamma-proteobacteria & AD6 & beta-proteobacteria & AD97 \\
\hline gamma-proteobacteria & AD6 & Flavobacteria & AD151 \\
\hline gamma-proteobacteria & AD6 & alpha-proteobacteria & AD140 \\
\hline gamma-proteobacteria & AD6 & beta-proteobacteria & AD62 \\
\hline gamma-proteobacteria & AD6 & beta-proteobacteria & $\mathrm{AD}_{39}$ \\
\hline gamma-proteobacteria & AD6 & beta-proteobacteria & AD8o \\
\hline
\end{tabular}


Chapter Two

Table S2.10 continuation

Phylogenetic class

gamma-proteobacteria gamma-proteobacteria gamma-proteobacteria gamma-proteobacteria gamma-proteobacteria gamma-proteobacteria gamma-proteobacteria gamma-proteobacteria gamma-proteobacteria gamma-proteobacteria gamma-proteobacteria gamma-proteobacteria gamma-proteobacteria gamma-proteobacteria gamma-proteobacteria gamma-proteobacteria gamma-proteobacteria gamma-proteobacteria gamma-proteobacteria gamma-proteobacteria gamma-proteobacteria gamma-proteobacteri gamma-proteobacteria gamma-proteobacteria gamma-proteobacteri gamma-proteobacteria beta-proteobacteria

beta-proteobacteria beta-proteobacteria beta-proteobacteria

beta-proteobacteria beta-proteobacteria beta-proteobacteria

beta-proteobacteria beta-proteobacteria beta-proteobacteria

beta-proteobacteria beta-proteobacteria beta-proteobacteria beta-proteobacteria beta-proteobacteria beta-proteobacteria beta-proteobacteria beta-proteobacteria beta-proteobacteria beta-proteobacteria beta-proteobacteria beta-proteobacteria gamma-proteobacteria gamma-proteobacteria gamma-proteobacteria gamma-proteobacteria gamma-proteobacteria gamma-proteobacteria gamma-proteobacteria gamma-proteobacteria gamma-proteobacteria gamma-proteobacteria gamma-proteobacteria gamma-proteobacteria gamma-proteobacteria

\begin{tabular}{|c|c|c|}
\hline Strain code genus $\mathrm{A}$ & Phylogenetic class & Strain code genus $B$ \\
\hline AD6 & beta-proteobacteria & AD103 \\
\hline AD6 & beta-proteobacteria & $\mathrm{AD}_{73}$ \\
\hline AD6 & beta-proteobacteria & $\mathrm{AD}_{7}$ \\
\hline AD8 & beta-proteobacteria & $\mathrm{AD}_{32}$ \\
\hline AD8 & beta-proteobacteria & $\mathrm{AD}_{5} 8$ \\
\hline AD8 & gamma-proteobacteria & $\mathrm{AD}_{4}$ \\
\hline AD8 & gamma-proteobacteria & $\mathrm{AD}_{5}$ \\
\hline AD8 & gamma-proteobacteria & AD79 \\
\hline AD8 & Bacilli & $\mathrm{ADB}_{3}$ \\
\hline AD8 & Flavobacteria & AD134 \\
\hline AD8 & Flavobacteria & AD142 \\
\hline AD8 & beta-proteobacteria & $\mathrm{AD}_{27}$ \\
\hline AD8 & beta-proteobacteria & $\mathrm{AD}_{24}$ \\
\hline AD8 & beta-proteobacteria & $A D_{28}$ \\
\hline AD8 & gamma-proteobacteria & $\mathrm{AD}_{52}$ \\
\hline AD8 & beta-proteobacteria & $\mathrm{AD}_{76}$ \\
\hline AD8 & gamma-proteobacteria & ADio4 \\
\hline AD8 & Flavobacteria & AD131 \\
\hline AD8 & alpha-proteobacteria & AD140 \\
\hline AD8 & beta-proteobacteria & $\mathrm{AD}_{30}$ \\
\hline AD8 & Flavobacteria & $\mathrm{AD}_{41}$ \\
\hline AD8 & alpha-proteobacteria & $\mathrm{AD}_{34}$ \\
\hline AD8 & beta-proteobacteria & AD81 \\
\hline AD8 & beta-proteobacteria & $\mathrm{AD}_{77}$ \\
\hline AD8 & Actinobacteria & AD12 \\
\hline AD8 & gamma-proteobacteria & $\mathrm{AD}_{2}$ \\
\hline AD9 & beta-proteobacteria & $\mathrm{AD}_{33}$ \\
\hline AD9 & beta-proteobacteria & $\mathrm{AD}_{59}$ \\
\hline AD9 & beta-proteobacteria & AD1o \\
\hline AD9 & beta-proteobacteria & AD8o \\
\hline AD9 & beta-proteobacteria & AD88 \\
\hline AD9 & alpha-proteobacteria & AD136 \\
\hline AD9 & Flavobacteria & AD91 \\
\hline AD9 & beta-proteobacteria & AD26 \\
\hline AD9 & beta-proteobacteria & $\mathrm{AD}_{32}$ \\
\hline AD9 & alpha-proteobacteria & $\mathrm{AD}_{34}$ \\
\hline AD9 & beta-proteobacteria & AD6o \\
\hline AD9 & beta-proteobacteria & AD81 \\
\hline AD9 & beta-proteobacteria & AD89 \\
\hline AD9 & beta-proteobacteria & AD137 \\
\hline AD9 & gamma-proteobacteria & AD157 \\
\hline AD9 & beta-proteobacteria & $\mathrm{AD}_{25}$ \\
\hline AD9 & Flavobacteria & $\mathrm{AD}_{42}$ \\
\hline AD9 & Flavobacteria & $\mathrm{AD}_{43}$ \\
\hline AD9 & beta-proteobacteria & AD82 \\
\hline AD9 & Bacilli & AD83 \\
\hline AD9 & Actinobacteria & $\mathrm{AD}_{31}$ \\
\hline AD9 & Bacilli & $\mathrm{AD}_{50}$ \\
\hline $\mathrm{AD}_{14}$ & gamma-proteobacteria & $\mathrm{AD}_{36}$ \\
\hline AD14 & beta-proteobacteria & AD62 \\
\hline $\mathrm{AD}_{14}$ & Bacilli & $\mathrm{ADB}_{3}$ \\
\hline $\mathrm{AD}_{14}$ & gamma-proteobacteria & AD1oo \\
\hline AD14 & alpha-proteobacteria & AD140 \\
\hline $\mathrm{AD}_{14}$ & beta-proteobacteria & AD144 \\
\hline AD14 & Actinobacteria & $\mathrm{AD}_{22}$ \\
\hline AD14 & gamma-proteobacteria & AD20 \\
\hline $\mathrm{AD}_{14}$ & Flavobacteria & $\mathrm{AD}_{44}$ \\
\hline $\mathrm{AD}_{14}$ & Flavobacteria & $\mathrm{AD}_{42}$ \\
\hline $\mathrm{AD}_{14}$ & beta-proteobacteria & AD68 \\
\hline AD14 & beta-proteobacteria & $\mathrm{AD}_{24}$ \\
\hline AD14 & beta-proteobacteria & AD95 \\
\hline
\end{tabular}




\section{Impact of interspecific interactions on antimicrobial activity among soil bacteria}

\begin{tabular}{|c|c|c|c|}
\hline Phylogenetic class & Strain code genus A & Phylogenetic class & Strain code genus B \\
\hline gamma-proteobacteria & AD14 & Bacilli & AD117 \\
\hline gamma-proteobacteria & AD14 & Flavobacteria & AD146 \\
\hline gamma-proteobacteria & AD14 & alpha-proteobacteria & AD159 \\
\hline gamma-proteobacteria & AD14 & gamma-proteobacteria & $\mathrm{AD}_{5} 6$ \\
\hline gamma-proteobacteria & AD14 & Bacilli & AD87 \\
\hline gamma-proteobacteria & AD14 & beta-proteobacteria & AD98 \\
\hline gamma-proteobacteria & AD14 & beta-proteobacteria & AD69 \\
\hline gamma-proteobacteria & $\mathrm{AD}_{14}$ & Actinobacteria & AD107 \\
\hline gamma-proteobacteria & $\mathrm{AD}_{14}$ & Actinobacteria & AD12 \\
\hline gamma-proteobacteria & AD16 & alpha-proteobacteria & $\mathrm{AD}_{3} 8$ \\
\hline gamma-proteobacteria & AD16 & beta-proteobacteria & AD64 \\
\hline gamma-proteobacteria & AD16 & gamma-proteobacteria & AD2o \\
\hline gamma-proteobacteria & AD16 & Bacilli & AD87 \\
\hline gamma-proteobacteria & AD16 & Bacilli & AD106 \\
\hline gamma-proteobacteria & AD16 & Flavobacteria & AD142 \\
\hline gamma-proteobacteria & AD16 & Flavobacteria & AD149 \\
\hline gamma-proteobacteria & AD16 & gamma-proteobacteria & $A_{21}$ \\
\hline gamma-proteobacteria & AD16 & beta-proteobacteria & AD19 \\
\hline gamma-proteobacteria & AD16 & Flavobacteria & $\mathrm{AD}_{4} 8$ \\
\hline gamma-proteobacteria & AD16 & Flavobacteria & $\mathrm{AD}_{41}$ \\
\hline gamma-proteobacteria & AD16 & beta-proteobacteria & $\mathrm{AD}_{73}$ \\
\hline gamma-proteobacteria & AD16 & beta-proteobacteria & AD67 \\
\hline gamma-proteobacteria & AD16 & beta-proteobacteria & $\mathrm{AD}_{25}$ \\
\hline gamma-proteobacteria & AD16 & Actinobacteria & AD94 \\
\hline gamma-proteobacteria & AD16 & beta-proteobacteria & AD96 \\
\hline gamma-proteobacteria & AD16 & beta-proteobacteria & AD145 \\
\hline gamma-proteobacteria & AD16 & Flavobacteria & AD151 \\
\hline gamma-proteobacteria & AD16 & beta-proteobacteria & AD54 \\
\hline gamma-proteobacteria & AD16 & beta-proteobacteria & $\mathrm{AD6}_{5}$ \\
\hline gamma-proteobacteria & AD16 & beta-proteobacteria & AD89 \\
\hline gamma-proteobacteria & AD16 & Bacilli & AD93 \\
\hline gamma-proteobacteria & AD16 & beta-proteobacteria & AD68 \\
\hline gamma-proteobacteria & AD16 & Actinobacteria & AD110 \\
\hline gamma-proteobacteria & AD16 & beta-proteobacteria & $\mathrm{AD}_{7}$ \\
\hline gamma-proteobacteria & AD17 & beta-proteobacteria & $\mathrm{AD}_{39}$ \\
\hline gamma-proteobacteria & AD17 & beta-proteobacteria & AD65 \\
\hline gamma-proteobacteria & $\mathrm{AD}_{17}$ & gamma-proteobacteria & AD21 \\
\hline gamma-proteobacteria & AD17 & Actinobacteria & $\mathrm{AD}_{92}$ \\
\hline gamma-proteobacteria & $\mathrm{AD}_{17}$ & alpha-proteobacteria & AD113 \\
\hline gamma-proteobacteria & AD17 & beta-proteobacteria & AD143 \\
\hline gamma-proteobacteria & $\mathrm{AD}_{17}$ & alpha-proteobacteria & AD153 \\
\hline gamma-proteobacteria & AD17 & beta-proteobacteria & AD19 \\
\hline gamma-proteobacteria & AD17 & beta-proteobacteria & AD18 \\
\hline gamma-proteobacteria & AD17 & alpha-proteobacteria & $A D_{51}$ \\
\hline gamma-proteobacteria & AD17 & gamma-proteobacteria & $\mathrm{AD}_{40}$ \\
\hline gamma-proteobacteria & AD17 & beta-proteobacteria & AD66 \\
\hline gamma-proteobacteria & $\mathrm{AD}_{17}$ & beta-proteobacteria & AD19 \\
\hline gamma-proteobacteria & $\mathrm{AD}_{17}$ & Bacilli & AD93 \\
\hline gamma-proteobacteria & AD17 & gamma-proteobacteria & AD114 \\
\hline gamma-proteobacteria & $\mathrm{AD}_{17}$ & beta-proteobacteria & $\mathrm{AD}_{144}$ \\
\hline gamma-proteobacteria & AD17 & alpha-proteobacteria & $\mathrm{AD}_{152}$ \\
\hline gamma-proteobacteria & $\mathrm{AD}_{17}$ & gamma-proteobacteria & $\mathrm{AD}_{52}$ \\
\hline gamma-proteobacteria & $\mathrm{AD}_{17}$ & Flavobacteria & AD91 \\
\hline gamma-proteobacteria & AD17 & beta-proteobacteria & AD67 \\
\hline gamma-proteobacteria & AD17 & beta-proteobacteria & AD118 \\
\hline gamma-proteobacteria & AD17 & beta-proteobacteria & AD119 \\
\hline beta-proteobacteria & AD19 & Flavobacteria & $\mathrm{AD}_{41}$ \\
\hline beta-proteobacteria & AD19 & beta-proteobacteria & $\mathrm{AD}_{7}$ \\
\hline beta-proteobacteria & AD19 & beta-proteobacteria & AD18 \\
\hline beta-proteobacteria & AD19 & beta-proteobacteria & $\mathrm{AD}_{25}$ \\
\hline beta-proteobacteria & AD19 & Actinobacteria & AD94 \\
\hline beta-proteobacteria & AD19 & beta-proteobacteria & AD96 \\
\hline
\end{tabular}


Table S2.10 continuation

\begin{tabular}{|c|c|c|c|}
\hline Phylogenetic class & Strain code genus A & Phylogenetic class & Strain code genus B \\
\hline beta-proteobacteria & AD19 & beta-proteobacteria & AD145 \\
\hline beta-proteobacteria & AD19 & Flavobacteria & AD151 \\
\hline beta-proteobacteria & AD19 & gamma-proteobacteria & AD16 \\
\hline beta-proteobacteria & AD19 & gamma-proteobacteria & AD17 \\
\hline beta-proteobacteria & AD19 & beta-proteobacteria & $\mathrm{AD}_{54}$ \\
\hline beta-proteobacteria & AD19 & gamma-proteobacteria & $\mathrm{AD}_{40}$ \\
\hline beta-proteobacteria & AD19 & beta-proteobacteria & $\mathrm{AD}_{39}$ \\
\hline beta-proteobacteria & AD19 & beta-proteobacteria & AD66 \\
\hline beta-proteobacteria & AD19 & beta-proteobacteria & $\mathrm{AD}_{5}$ \\
\hline beta-proteobacteria & AD19 & Bacilli & AD93 \\
\hline beta-proteobacteria & AD19 & gamma-proteobacteria & AD114 \\
\hline beta-proteobacteria & AD19 & beta-proteobacteria & AD144 \\
\hline beta-proteobacteria & AD19 & alpha-proteobacteria & AD152 \\
\hline beta-proteobacteria & AD19 & gamma-proteobacteria & AD21 \\
\hline beta-proteobacteria & AD19 & gamma-proteobacteria & $\mathrm{AD}_{52}$ \\
\hline beta-proteobacteria & AD19 & Actinobacteria & AD92 \\
\hline beta-proteobacteria & AD19 & alpha-proteobacteria & AD113 \\
\hline beta-proteobacteria & AD19 & beta-proteobacteria & AD143 \\
\hline beta-proteobacteria & AD19 & alpha-proteobacteria & AD153 \\
\hline beta-proteobacteria & AD19 & alpha-proteobacteria & $A_{51}$ \\
\hline beta-proteobacteria & AD19 & beta-proteobacteria & AD68 \\
\hline beta-proteobacteria & AD19 & Flavobacteria & AD91 \\
\hline beta-proteobacteria & AD19 & beta-proteobacteria & $\mathrm{AD}_{7}$ \\
\hline beta-proteobacteria & AD19 & beta-proteobacteria & AD119 \\
\hline beta-proteobacteria & AD19 & beta-proteobacteria & AD118 \\
\hline beta-proteobacteria & $\mathrm{AD}_{35}$ & beta-proteobacteria & AD11 \\
\hline beta-proteobacteria & $\mathrm{AD}_{35}$ & beta-proteobacteria & AD61 \\
\hline beta-proteobacteria & $\mathrm{AD}_{35}$ & beta-proteobacteria & AD15 \\
\hline beta-proteobacteria & $\mathrm{AD}_{35}$ & beta-proteobacteria & AD82 \\
\hline beta-proteobacteria & $\mathrm{AD}_{35}$ & beta-proteobacteria & AD98 \\
\hline beta-proteobacteria & $\mathrm{AD}_{35}$ & beta-proteobacteria & $\mathrm{AD}_{138}$ \\
\hline beta-proteobacteria & $\mathrm{AD}_{35}$ & beta-proteobacteria & AD143 \\
\hline beta-proteobacteria & $\mathrm{AD}_{35}$ & beta-proteobacteria & $\mathrm{AD}_{23}$ \\
\hline beta-proteobacteria & $\mathrm{AD}_{35}$ & beta-proteobacteria & AD61 \\
\hline beta-proteobacteria & $\mathrm{AD}_{35}$ & Flavobacteria & $\mathrm{AD}_{45}$ \\
\hline beta-proteobacteria & $\mathrm{AD}_{35}$ & Flavobacteria & AD86 \\
\hline beta-proteobacteria & $\mathrm{AD}_{35}$ & beta-proteobacteria & $A_{55}$ \\
\hline beta-proteobacteria & $\mathrm{AD}_{39}$ & gamma-proteobacteria & AD17 \\
\hline beta-proteobacteria & $\mathrm{AD}_{39}$ & beta-proteobacteria & AD26 \\
\hline beta-proteobacteria & $\mathrm{AD}_{39}$ & beta-proteobacteria & AD65 \\
\hline beta-proteobacteria & $\mathrm{AD}_{39}$ & gamma-proteobacteria & $A_{21}$ \\
\hline beta-proteobacteria & $\mathrm{AD}_{39}$ & alpha-proteobacteria & $\mathrm{AD}_{51}$ \\
\hline beta-proteobacteria & $\mathrm{AD}_{39}$ & Actinobacteria & $\mathrm{AD}_{92}$ \\
\hline beta-proteobacteria & $\mathrm{AD}_{39}$ & alpha-proteobacteria & AD113 \\
\hline beta-proteobacteria & $\mathrm{AD}_{39}$ & beta-proteobacteria & AD143 \\
\hline beta-proteobacteria & $\mathrm{AD}_{39}$ & alpha-proteobacteria & AD153 \\
\hline beta-proteobacteria & $\mathrm{AD}_{39}$ & beta-proteobacteria & AD19 \\
\hline beta-proteobacteria & $\mathrm{AD}_{39}$ & alpha-proteobacteria & $\mathrm{AD}_{51}$ \\
\hline beta-proteobacteria & $\mathrm{AD}_{39}$ & beta-proteobacteria & $\mathrm{AD}_{74}$ \\
\hline beta-proteobacteria & $\mathrm{AD}_{39}$ & beta-proteobacteria & $\mathrm{AD}_{103}$ \\
\hline beta-proteobacteria & $\mathrm{AD}_{39}$ & gamma-proteobacteria & AD120 \\
\hline beta-proteobacteria & $\mathrm{AD}_{39}$ & beta-proteobacteria & AD137 \\
\hline beta-proteobacteria & $\mathrm{AD}_{39}$ & gamma-proteobacteria & $\mathrm{AD}_{4}$ \\
\hline beta-proteobacteria & $\mathrm{AD}_{39}$ & beta-proteobacteria & AD64 \\
\hline beta-proteobacteria & $\mathrm{AD}_{39}$ & gamma-proteobacteria & AD6 \\
\hline beta-proteobacteria & $\mathrm{AD}_{39}$ & beta-proteobacteria & AD66 \\
\hline beta-proteobacteria & $\mathrm{AD}_{39}$ & beta-proteobacteria & $\mathrm{AD}_{30}$ \\
\hline beta-proteobacteria & $\mathrm{AD}_{39}$ & gamma-proteobacteria & $\mathrm{AD}_{5} 6$ \\
\hline beta-proteobacteria & $\mathrm{AD}_{39}$ & beta-proteobacteria & $\mathrm{AD}_{76}$ \\
\hline beta-proteobacteria & $\mathrm{AD}_{39}$ & Bacilli & $\mathrm{AD}_{78}$ \\
\hline beta-proteobacteria & $\mathrm{AD}_{39}$ & beta-proteobacteria & AD81 \\
\hline beta-proteobacteria & $\mathrm{AD}_{39}$ & beta-proteobacteria & AD133 \\
\hline
\end{tabular}




\section{Impact of interspecific interactions on antimicrobial activity among soil bacteria}

\begin{tabular}{|c|c|c|c|}
\hline Phylogenetic class & Strain code genus A & Phylogenetic class & Strain code genus B \\
\hline beta-proteobacteria & $\mathrm{AD}_{39}$ & Actinobacteria & AD141 \\
\hline beta-proteobacteria & $\mathrm{AD}_{39}$ & beta-proteobacteria & AD11 \\
\hline beta-proteobacteria & $\mathrm{AD}_{39}$ & Flavobacteria & AD91 \\
\hline beta-proteobacteria & $\mathrm{AD}_{39}$ & gamma-proteobacteria & AD105 \\
\hline beta-proteobacteria & $\mathrm{AD}_{39}$ & beta-proteobacteria & $\mathrm{AD}_{28}$ \\
\hline beta-proteobacteria & $\mathrm{AD}_{39}$ & beta-proteobacteria & AD118 \\
\hline beta-proteobacteria & $\mathrm{AD}_{39}$ & Actinobacteria & AD110 \\
\hline beta-proteobacteria & $\mathrm{AD}_{54}$ & gamma-proteobacteria & $\mathrm{AD}_{5}$ \\
\hline beta-proteobacteria & $\mathrm{AD}_{54}$ & beta-proteobacteria & AD19 \\
\hline beta-proteobacteria & $\mathrm{AD}_{54}$ & Actinobacteria & AD29 \\
\hline beta-proteobacteria & $\mathrm{AD}_{54}$ & Flavobacteria & $\mathrm{AD}_{41}$ \\
\hline beta-proteobacteria & $\mathrm{AD}_{54}$ & gamma-proteobacteria & $\mathrm{AD}_{4}$ \\
\hline beta-proteobacteria & $\mathrm{AD}_{54}$ & beta-proteobacteria & $\mathrm{AD}_{77}$ \\
\hline beta-proteobacteria & AD54 & gamma-proteobacteria & AD105 \\
\hline beta-proteobacteria & $\mathrm{AD}_{54}$ & alpha-proteobacteria & AD132 \\
\hline beta-proteobacteria & $\mathrm{AD}_{54}$ & beta-proteobacteria & AD145 \\
\hline beta-proteobacteria & $\mathrm{AD}_{54}$ & beta-proteobacteria & $\mathrm{AD}_{7}$ \\
\hline beta-proteobacteria & AD54 & beta-proteobacteria & ADoo9 \\
\hline beta-proteobacteria & AD54 & beta-proteobacteria & AD25 \\
\hline beta-proteobacteria & $\mathrm{AD}_{54}$ & Actinobacteria & AD94 \\
\hline beta-proteobacteria & $\mathrm{AD}_{54}$ & beta-proteobacteria & AD96 \\
\hline beta-proteobacteria & $\mathrm{AD}_{54}$ & Flavobacteria & AD151 \\
\hline beta-proteobacteria & $\mathrm{AD}_{54}$ & gamma-proteobacteria & AD16 \\
\hline beta-proteobacteria & $\mathrm{AD}_{54}$ & beta-proteobacteria & $\mathrm{AD}_{37}$ \\
\hline beta-proteobacteria & $\mathrm{AD}_{54}$ & beta-proteobacteria & AD68 \\
\hline beta-proteobacteria & $\mathrm{AD}_{54}$ & Bacilli & $\mathrm{AD}_{\mathbf{7}} \mathbf{8}$ \\
\hline beta-proteobacteria & $\mathrm{AD}_{54}$ & beta-proteobacteria & $\mathrm{AD}_{3}$ \\
\hline beta-proteobacteria & $\mathrm{AD}_{54}$ & beta-proteobacteria & $\mathrm{AD}_{7}$ \\
\hline beta-proteobacteria & AD64 & gamma-proteobacteria & AD16 \\
\hline beta-proteobacteria & AD64 & beta-proteobacteria & AD26 \\
\hline beta-proteobacteria & AD64 & alpha-proteobacteria & $\mathrm{AD}_{3} 8$ \\
\hline beta-proteobacteria & AD64 & alpha-proteobacteria & $\mathrm{AD}_{51}$ \\
\hline beta-proteobacteria & AD64 & gamma-proteobacteria & AD2o \\
\hline beta-proteobacteria & AD64 & Bacilli & AD87 \\
\hline beta-proteobacteria & AD64 & Bacilli & AD106 \\
\hline beta-proteobacteria & AD64 & Flavobacteria & AD142 \\
\hline beta-proteobacteria & AD64 & Flavobacteria & AD149 \\
\hline beta-proteobacteria & AD64 & gamma-proteobacteria & $\mathrm{AD}_{21}$ \\
\hline beta-proteobacteria & AD64 & beta-proteobacteria & $\mathrm{AD}_{74}$ \\
\hline beta-proteobacteria & AD64 & Flavobacteria & $\mathrm{AD}_{4} 8$ \\
\hline beta-proteobacteria & AD64 & beta-proteobacteria & $\mathrm{AD}_{39}$ \\
\hline beta-proteobacteria & AD64 & beta-proteobacteria & $\mathrm{AD}_{103}$ \\
\hline beta-proteobacteria & AD64 & gamma-proteobacteria & AD120 \\
\hline beta-proteobacteria & AD64 & alpha-proteobacteria & AD153 \\
\hline beta-proteobacteria & AD64 & beta-proteobacteria & AD137 \\
\hline beta-proteobacteria & AD64 & gamma-proteobacteria & $\mathrm{AD}_{4}$ \\
\hline beta-proteobacteria & AD64 & beta-proteobacteria & AD65 \\
\hline beta-proteobacteria & AD64 & beta-proteobacteria & $\mathrm{AD}_{76}$ \\
\hline beta-proteobacteria & AD64 & beta-proteobacteria & AD89 \\
\hline beta-proteobacteria & AD64 & beta-proteobacteria & AD123 \\
\hline beta-proteobacteria & AD64 & Actinobacteria & AD110 \\
\hline beta-proteobacteria & AD66 & beta-proteobacteria & AD18 \\
\hline beta-proteobacteria & AD66 & gamma-proteobacteria & $\mathrm{AD}_{4} \mathrm{O}$ \\
\hline beta-proteobacteria & AD66 & beta-proteobacteria & AD19 \\
\hline beta-proteobacteria & AD66 & Bacilli & AD93 \\
\hline beta-proteobacteria & AD66 & gamma-proteobacteria & AD114 \\
\hline beta-proteobacteria & AD66 & beta-proteobacteria & AD144 \\
\hline beta-proteobacteria & AD66 & alpha-proteobacteria & AD152 \\
\hline beta-proteobacteria & AD66 & gamma-proteobacteria & AD17 \\
\hline beta-proteobacteria & AD66 & gamma-proteobacteria & $\mathrm{AD}_{52}$ \\
\hline beta-proteobacteria & AD66 & beta-proteobacteria & $\mathrm{AD}_{39}$ \\
\hline beta-proteobacteria & AD66 & beta-proteobacteria & AD67 \\
\hline
\end{tabular}


Chapter Two

Table S2.10 continuation

\begin{tabular}{|c|c|c|c|}
\hline Phylogenetic class & Strain code genus $\mathrm{A}$ & Phylogenetic class & Strain code genus $B$ \\
\hline beta-proteobacteria & AD66 & beta-proteobacteria & AD65 \\
\hline beta-proteobacteria & AD66 & gamma-proteobacteria & AD21 \\
\hline beta-proteobacteria & AD66 & Actinobacteria & AD92 \\
\hline beta-proteobacteria & AD66 & alpha-proteobacteria & AD113 \\
\hline beta-proteobacteria & AD66 & beta-proteobacteria & AD143 \\
\hline beta-proteobacteria & AD66 & alpha-proteobacteria & AD153 \\
\hline beta-proteobacteria & AD66 & alpha-proteobacteria & $A D_{51}$ \\
\hline beta-proteobacteria & AD66 & gamma-proteobacteria & AD125 \\
\hline beta-proteobacteria & AD66 & beta-proteobacteria & AD119 \\
\hline gamma-proteobacteria & $\mathrm{AD}_{79}$ & gamma-proteobacteria & AD8 \\
\hline gamma-proteobacteria & $\mathrm{AD}_{79}$ & beta-proteobacteria & $\mathrm{AD}_{32}$ \\
\hline gamma-proteobacteria & $\mathrm{AD}_{79}$ & beta-proteobacteria & $\mathrm{AD}_{5} 8$ \\
\hline gamma-proteobacteria & $\mathrm{AD}_{79}$ & gamma-proteobacteria & $\mathrm{AD}_{5}$ \\
\hline gamma-proteobacteria & $\mathrm{AD}_{79}$ & Bacilli & $\mathrm{ADB}_{3}$ \\
\hline gamma-proteobacteria & $\mathrm{AD}_{79}$ & Flavobacteria & AD134 \\
\hline gamma-proteobacteria & $\mathrm{AD}_{79}$ & Flavobacteria & AD142 \\
\hline gamma-proteobacteria & $\mathrm{AD}_{79}$ & beta-proteobacteria & $\mathrm{AD}_{27}$ \\
\hline gamma-proteobacteria & $\mathrm{AD}_{79}$ & beta-proteobacteria & $\mathrm{AD}_{24}$ \\
\hline gamma-proteobacteria & $\mathrm{AD}_{79}$ & Flavobacteria & $\mathrm{AD}_{41}$ \\
\hline gamma-proteobacteria & $\mathrm{AD}_{79}$ & beta-proteobacteria & AD81 \\
\hline gamma-proteobacteria & $\mathrm{AD}_{79}$ & beta-proteobacteria & AD130 \\
\hline gamma-proteobacteria & $\mathrm{AD}_{79}$ & Actinobacteria & AD12 \\
\hline beta-proteobacteria & AD81 & beta-proteobacteria & AD1o \\
\hline beta-proteobacteria & AD81 & gamma-proteobacteria & AD6 \\
\hline beta-proteobacteria & AD81 & alpha-proteobacteria & $\mathrm{AD}_{34}$ \\
\hline beta-proteobacteria & AD81 & beta-proteobacteria & $\mathrm{AD}_{30}$ \\
\hline beta-proteobacteria & AD81 & beta-proteobacteria & AD6o \\
\hline beta-proteobacteria & AD81 & gamma-proteobacteria & $\mathrm{AD}_{5} 6$ \\
\hline beta-proteobacteria & AD81 & beta-proteobacteria & AD9 \\
\hline beta-proteobacteria & AD81 & Bacilli & $\mathrm{AD}_{7} 8$ \\
\hline beta-proteobacteria & AD81 & beta-proteobacteria & AD89 \\
\hline beta-proteobacteria & AD81 & beta-proteobacteria & AD137 \\
\hline beta-proteobacteria & AD81 & gamma-proteobacteria & AD157 \\
\hline beta-proteobacteria & AD81 & beta-proteobacteria & $\mathrm{AD}_{25}$ \\
\hline beta-proteobacteria & AD81 & beta-proteobacteria & $\mathrm{AD}_{33}$ \\
\hline beta-proteobacteria & AD81 & beta-proteobacteria & AD133 \\
\hline beta-proteobacteria & AD81 & Actinobacteria & AD141 \\
\hline beta-proteobacteria & AD81 & beta-proteobacteria & AD28 \\
\hline beta-proteobacteria & AD81 & beta-proteobacteria & AD11 \\
\hline beta-proteobacteria & AD81 & Flavobacteria & $\mathrm{AD}_{43}$ \\
\hline beta-proteobacteria & AD81 & beta-proteobacteria & $A_{39}$ \\
\hline beta-proteobacteria & AD81 & beta-proteobacteria & AD89 \\
\hline beta-proteobacteria & AD81 & beta-proteobacteria & $\mathrm{AD}_{32}$ \\
\hline beta-proteobacteria & AD81 & beta-proteobacteria & AD 58 \\
\hline beta-proteobacteria & AD81 & gamma-proteobacteria & $\mathrm{AD}_{5}$ \\
\hline beta-proteobacteria & AD81 & Bacilli & $\mathrm{AD8}_{3}$ \\
\hline beta-proteobacteria & AD81 & gamma-proteobacteria & $\mathrm{AD}_{79}$ \\
\hline beta-proteobacteria & AD81 & beta-proteobacteria & AD8o \\
\hline beta-proteobacteria & AD81 & Flavobacteria & AD134 \\
\hline beta-proteobacteria & AD81 & Flavobacteria & $A_{142}$ \\
\hline beta-proteobacteria & AD81 & beta-proteobacteria & $\mathrm{AD}_{27}$ \\
\hline beta-proteobacteria & AD81 & Bacilli & $\mathrm{AD}_{50}$ \\
\hline beta-proteobacteria & AD81 & beta-proteobacteria & $\mathrm{AD}_{7}$ \\
\hline beta-proteobacteria & AD81 & Actinobacteria & AD12 \\
\hline beta-proteobacteria & AD82 & beta-proteobacteria & AD11 \\
\hline beta-proteobacteria & AD82 & beta-proteobacteria & $\mathrm{AD}_{35}$ \\
\hline beta-proteobacteria & AD82 & beta-proteobacteria & AD61 \\
\hline beta-proteobacteria & AD82 & beta-proteobacteria & AD15 \\
\hline beta-proteobacteria & AD82 & beta-proteobacteria & AD98 \\
\hline beta-proteobacteria & AD82 & beta-proteobacteria & $\mathrm{AD}_{138} 8$ \\
\hline beta-proteobacteria & AD82 & beta-proteobacteria & AD143 \\
\hline
\end{tabular}




\section{Impact of interspecific interactions on antimicrobial activity among soil bacteria}

\begin{tabular}{|c|c|c|c|}
\hline Phylogenetic class & Strain code genus A & Phylogenetic class & Strain code genus B \\
\hline beta-proteobacteria & AD82 & beta-proteobacteria & $\mathrm{AD}_{23}$ \\
\hline beta-proteobacteria & AD82 & Flavobacteria & $\mathrm{AD}_{45}$ \\
\hline beta-proteobacteria & AD82 & beta-proteobacteria & AD9 \\
\hline beta-proteobacteria & AD82 & beta-proteobacteria & $\mathrm{AD}_{33}$ \\
\hline beta-proteobacteria & AD82 & beta-proteobacteria & $\mathrm{AD}_{59}$ \\
\hline beta-proteobacteria & AD82 & beta-proteobacteria & AD1o \\
\hline beta-proteobacteria & AD82 & Flavobacteria & AD86 \\
\hline beta-proteobacteria & AD82 & beta-proteobacteria & AD8o \\
\hline beta-proteobacteria & AD82 & beta-proteobacteria & AD88 \\
\hline beta-proteobacteria & AD82 & alpha-proteobacteria & AD136 \\
\hline beta-proteobacteria & AD82 & Flavobacteria & AD91 \\
\hline beta-proteobacteria & AD82 & beta-proteobacteria & AD26 \\
\hline beta-proteobacteria & AD82 & beta-proteobacteria & $\mathrm{AD}_{55}$ \\
\hline beta-proteobacteria & AD82 & Actinobacteria & $\mathrm{AD}_{31}$ \\
\hline beta-proteobacteria & AD103 & beta-proteobacteria & AD26 \\
\hline beta-proteobacteria & AD103 & alpha-proteobacteria & $A_{51}$ \\
\hline beta-proteobacteria & AD103 & beta-proteobacteria & $\mathrm{AD}_{74}$ \\
\hline beta-proteobacteria & AD103 & beta-proteobacteria & $\mathrm{AD}_{39}$ \\
\hline beta-proteobacteria & AD103 & gamma-proteobacteria & AD12o \\
\hline beta-proteobacteria & AD103 & alpha-proteobacteria & AD153 \\
\hline beta-proteobacteria & AD103 & beta-proteobacteria & AD137 \\
\hline beta-proteobacteria & AD103 & gamma-proteobacteria & $\mathrm{AD}_{4}$ \\
\hline beta-proteobacteria & AD103 & beta-proteobacteria & AD64 \\
\hline beta-proteobacteria & AD103 & beta-proteobacteria & $\mathrm{AD}_{76}$ \\
\hline beta-proteobacteria & AD103 & beta-proteobacteria & $\mathrm{AD}_{24}$ \\
\hline beta-proteobacteria & AD103 & gamma-proteobacteria & $\mathrm{AD}_{46}$ \\
\hline beta-proteobacteria & AD103 & beta-proteobacteria & $\mathrm{AD}_{72}$ \\
\hline beta-proteobacteria & AD103 & beta-proteobacteria & $\mathrm{AD}_{32}$ \\
\hline beta-proteobacteria & AD103 & gamma-proteobacteria & AD105 \\
\hline beta-proteobacteria & AD103 & beta-proteobacteria & AD99 \\
\hline beta-proteobacteria & AD103 & beta-proteobacteria & AD97 \\
\hline beta-proteobacteria & AD103 & Flavobacteria & AD151 \\
\hline beta-proteobacteria & AD103 & alpha-proteobacteria & AD140 \\
\hline beta-proteobacteria & AD103 & gamma-proteobacteria & AD6 \\
\hline beta-proteobacteria & AD103 & Actinobacteria & ADno \\
\hline beta-proteobacteria & AD103 & Actinobacteria & AD107 \\
\hline beta-proteobacteria & AD144 & beta-proteobacteria & AD18 \\
\hline beta-proteobacteria & AD144 & gamma-proteobacteria & AD14 \\
\hline beta-proteobacteria & AD144 & gamma-proteobacteria & $\mathrm{AD}_{40}$ \\
\hline beta-proteobacteria & AD144 & gamma-proteobacteria & $\mathrm{AD}_{36}$ \\
\hline beta-proteobacteria & AD144 & beta-proteobacteria & AD66 \\
\hline beta-proteobacteria & AD144 & beta-proteobacteria & AD62 \\
\hline beta-proteobacteria & AD144 & beta-proteobacteria & AD19 \\
\hline beta-proteobacteria & AD144 & Bacilli & AD93 \\
\hline beta-proteobacteria & $\mathrm{AD}_{144}$ & Bacilli & AD83 \\
\hline beta-proteobacteria & AD144 & gamma-proteobacteria & ADn14 \\
\hline beta-proteobacteria & AD144 & gamma-proteobacteria & ADioo \\
\hline beta-proteobacteria & AD144 & alpha-proteobacteria & AD14o \\
\hline beta-proteobacteria & $\mathrm{AD}_{144}$ & alpha-proteobacteria & AD152 \\
\hline beta-proteobacteria & AD144 & gamma-proteobacteria & AD17 \\
\hline beta-proteobacteria & AD144 & gamma-proteobacteria & $\mathrm{AD}_{52}$ \\
\hline beta-proteobacteria & AD144 & Actinobacteria & $\mathrm{AD}_{22}$ \\
\hline beta-proteobacteria & $\mathrm{AD}_{144}$ & Flavobacteria & $\mathrm{AD}_{44}$ \\
\hline beta-proteobacteria & AD144 & beta-proteobacteria & AD67 \\
\hline beta-proteobacteria & AD144 & Actinobacteria & AD92 \\
\hline beta-proteobacteria & AD144 & Bacilli & $\mathrm{AD8}_{7}$ \\
\hline gamma-proteobacteria & AD2o & Flavobacteria & $\mathrm{AD}_{42}$ \\
\hline gamma-proteobacteria & AD2o & beta-proteobacteria & AD68 \\
\hline gamma-proteobacteria & AD2o & gamma-proteobacteria & AD16 \\
\hline gamma-proteobacteria & AD2o & beta-proteobacteria & AD24 \\
\hline gamma-proteobacteria & AD2o & beta-proteobacteria & AD95 \\
\hline
\end{tabular}


Table S2.1o continuation

\begin{tabular}{|c|c|c|c|}
\hline Phylogenetic class & Strain code genus A & Phylogenetic class & Strain code genus $B$ \\
\hline gamma-proteobacteria & AD2o & Bacilli & AD117 \\
\hline gamma-proteobacteria & AD2o & Flavobacteria & AD146 \\
\hline gamma-proteobacteria & AD2o & alpha-proteobacteria & AD159 \\
\hline gamma-proteobacteria & AD2o & gamma-proteobacteria & $\mathrm{AD}_{14}$ \\
\hline gamma-proteobacteria & AD2o & beta-proteobacteria & $\mathrm{AD}_{15}$ \\
\hline gamma-proteobacteria & AD2o & gamma-proteobacteria & $\mathrm{AD}_{56}$ \\
\hline gamma-proteobacteria & AD2o & alpha-proteobacteria & $\mathrm{AD}_{3} 8$ \\
\hline gamma-proteobacteria & AD2o & beta-proteobacteria & $\mathrm{AD}_{37}$ \\
\hline gamma-proteobacteria & AD2o & beta-proteobacteria & $\mathrm{AD6}_{4}$ \\
\hline gamma-proteobacteria & $\mathrm{AD}_{20}$ & beta-proteobacteria & $\mathrm{AD}_{74}$ \\
\hline gamma-proteobacteria & AD2o & beta-proteobacteria & AD63 \\
\hline gamma-proteobacteria & AD2o & Bacilli & AD87 \\
\hline gamma-proteobacteria & AD2o & Bacilli & AD1o6 \\
\hline gamma-proteobacteria & AD2o & Flavobacteria & $\mathrm{AD}_{142}$ \\
\hline gamma-proteobacteria & AD2o & Flavobacteria & AD149 \\
\hline gamma-proteobacteria & AD2o & beta-proteobacteria & $\mathrm{AD}_{27}$ \\
\hline gamma-proteobacteria & AD2o & gamma-proteobacteria & AD21 \\
\hline gamma-proteobacteria & AD2o & Flavobacteria & $\mathrm{AD}_{4} 8$ \\
\hline gamma-proteobacteria & AD2o & Flavobacteria & $\mathrm{AD}_{4}$ \\
\hline gamma-proteobacteria & AD2o & beta-proteobacteria & AD1o1 \\
\hline gamma-proteobacteria & AD2o & Actinobacteria & AD141 \\
\hline gamma-proteobacteria & AD2o & Flavobacteria & $\mathrm{AD}_{47}$ \\
\hline gamma-proteobacteria & AD2o & beta-proteobacteria & AD69 \\
\hline gamma-proteobacteria & AD2o & beta-proteobacteria & $\mathrm{AD}_{5}$ \\
\hline gamma-proteobacteria & AD2o & beta-proteobacteria & AD98 \\
\hline gamma-proteobacteria & AD2o & beta-proteobacteria & AD89 \\
\hline gamma-proteobacteria & AD2o & beta-proteobacteria & AD88 \\
\hline gamma-proteobacteria & AD2o & Actinobacteria & AD12 \\
\hline gamma-proteobacteria & AD2o & Actinobacteria & AD110 \\
\hline gamma-proteobacteria & AD2o & Actinobacteria & AD1o8 \\
\hline gamma-proteobacteria & $\mathrm{AD}_{40}$ & beta-proteobacteria & AD18 \\
\hline gamma-proteobacteria & $\mathrm{AD}_{40} \mathrm{O}$ & beta-proteobacteria & AD66 \\
\hline gamma-proteobacteria & $\mathrm{AD}_{40} \mathrm{O}$ & beta-proteobacteria & AD19 \\
\hline gamma-proteobacteria & $\mathrm{AD}_{40}$ & Bacilli & AD93 \\
\hline gamma-proteobacteria & $\mathrm{AD}_{4} \mathrm{O}$ & gamma-proteobacteria & AD114 \\
\hline gamma-proteobacteria & $\mathrm{AD}_{40}$ & beta-proteobacteria & AD144 \\
\hline gamma-proteobacteria & $\mathrm{AD}_{40}$ & alpha-proteobacteria & AD152 \\
\hline gamma-proteobacteria & $\mathrm{AD}_{4} \mathrm{O}$ & gamma-proteobacteria & AD17 \\
\hline gamma-proteobacteria & $\mathrm{AD}_{40}$ & gamma-proteobacteria & $A_{52}$ \\
\hline gamma-proteobacteria & $\mathrm{AD}_{4} \mathrm{O}$ & beta-proteobacteria & AD67 \\
\hline gamma-proteobacteria & $\mathrm{AD}_{40}$ & Actinobacteria & AD92 \\
\hline gamma-proteobacteria & $\mathrm{AD}_{4} \mathrm{O}$ & beta-proteobacteria & AD119 \\
\hline gamma-proteobacteria & $\mathrm{AD}_{52}$ & gamma-proteobacteria & $\mathrm{AD}_{4}$ \\
\hline gamma-proteobacteria & $\mathrm{AD}_{52}$ & beta-proteobacteria & AD18 \\
\hline gamma-proteobacteria & $\mathrm{AD}_{52}$ & beta-proteobacteria & $\mathrm{AD}_{28} 8$ \\
\hline gamma-proteobacteria & $\mathrm{AD}_{52}$ & gamma-proteobacteria & $\mathrm{AD}_{40}$ \\
\hline gamma-proteobacteria & $\mathrm{AD}_{52}$ & gamma-proteobacteria & AD8 \\
\hline gamma-proteobacteria & $\mathrm{AD}_{52}$ & beta-proteobacteria & $\mathrm{AD}_{76}$ \\
\hline gamma-proteobacteria & $\mathrm{AD}_{52}$ & gamma-proteobacteria & AD104 \\
\hline gamma-proteobacteria & $A_{52}$ & Flavobacteria & $A_{131}$ \\
\hline gamma-proteobacteria & $\mathrm{AD}_{52}$ & alpha-proteobacteria & AD140 \\
\hline gamma-proteobacteria & $\mathrm{AD}_{52}$ & beta-proteobacteria & $\mathrm{AD}_{30}$ \\
\hline gamma-proteobacteria & $\mathrm{AD}_{52}$ & beta-proteobacteria & AD66 \\
\hline gamma-proteobacteria & $\mathrm{AD}_{52}$ & gamma-proteobacteria & AD8 \\
\hline gamma-proteobacteria & $\mathrm{AD}_{52}$ & beta-proteobacteria & AD19 \\
\hline gamma-proteobacteria & $\mathrm{AD}_{52}$ & Bacilli & AD93 \\
\hline gamma-proteobacteria & $\mathrm{AD}_{52}$ & gamma-proteobacteria & AD114 \\
\hline gamma-proteobacteria & $\mathrm{AD}_{52}$ & beta-proteobacteria & $\mathrm{AD}_{144}$ \\
\hline gamma-proteobacteria & $\mathrm{AD}_{52}$ & alpha-proteobacteria & AD152 \\
\hline gamma-proteobacteria & $\mathrm{AD}_{52}$ & gamma-proteobacteria & $\mathrm{AD}_{17}$ \\
\hline gamma-proteobacteria & $\mathrm{AD}_{52}$ & alpha-proteobacteria & $\mathrm{AD}_{34}$ \\
\hline gamma-proteobacteria & $\mathrm{AD}_{52}$ & beta-proteobacteria & AD67 \\
\hline
\end{tabular}




\section{Impact of interspecific interactions on antimicrobial activity among soil bacteria}

\begin{tabular}{|c|c|c|c|}
\hline Phylogenetic class & Strain code genus A & Phylogenetic class & Strain code genus B \\
\hline gamma-proteobacteria & $\mathrm{AD}_{52}$ & beta-proteobacteria & $\mathrm{AD}_{77}$ \\
\hline gamma-proteobacteria & $\mathrm{AD}_{52}$ & gamma-proteobacteria & AD2 \\
\hline alpha-proteobacteria & $\mathrm{AD}_{51}$ & beta-proteobacteria & AD26 \\
\hline alpha-proteobacteria & $A D_{51}$ & gamma-proteobacteria & AD17 \\
\hline alpha-proteobacteria & $\mathrm{AD}_{51}$ & beta-proteobacteria & $\mathrm{AD}_{74}$ \\
\hline alpha-proteobacteria & $\mathrm{AD}_{51}$ & beta-proteobacteria & $\mathrm{AD}_{39}$ \\
\hline alpha-proteobacteria & $A D_{51}$ & beta-proteobacteria & AD103 \\
\hline alpha-proteobacteria & $\mathrm{AD}_{51}$ & gamma-proteobacteria & AD120 \\
\hline alpha-proteobacteria & $A_{51}$ & alpha-proteobacteria & $\mathrm{AD}_{153}$ \\
\hline alpha-proteobacteria & $\mathrm{AD}_{51}$ & beta-proteobacteria & AD137 \\
\hline alpha-proteobacteria & $\mathrm{AD}_{51}$ & gamma-proteobacteria & $\mathrm{AD}_{4}$ \\
\hline alpha-proteobacteria & $\mathrm{AD}_{51}$ & beta-proteobacteria & $\mathrm{AD}_{39}$ \\
\hline alpha-proteobacteria & AD51 & beta-proteobacteria & AD64 \\
\hline alpha-proteobacteria & $\mathrm{AD}_{51}$ & beta-proteobacteria & AD65 \\
\hline alpha-proteobacteria & $A D_{51}$ & gamma-proteobacteria & AD21 \\
\hline alpha-proteobacteria & $\mathrm{AD}_{51}$ & Actinobacteria & AD92 \\
\hline alpha-proteobacteria & $\mathrm{AD}_{51}$ & alpha-proteobacteria & AD113 \\
\hline alpha-proteobacteria & $\mathrm{AD}_{51}$ & beta-proteobacteria & AD143 \\
\hline alpha-proteobacteria & $A_{51}$ & beta-proteobacteria & AD19 \\
\hline alpha-proteobacteria & $\mathrm{AD}_{51}$ & beta-proteobacteria & $\mathrm{AD}_{7} 6$ \\
\hline alpha-proteobacteria & $\mathrm{AD}_{51}$ & beta-proteobacteria & AD66 \\
\hline alpha-proteobacteria & $\mathrm{AD}_{51}$ & gamma-proteobacteria & AD105 \\
\hline alpha-proteobacteria & $A_{51}$ & beta-proteobacteria & AD118 \\
\hline
\end{tabular}




\section{Chapter 3}

\section{Volatiles in interspecific bacterial interactions}

Olaf Tyc, Hans Zweers, Wietse de Boer and Paolina Garbeva

This chapter has been published as:

Tyc, O., Zweers, H., De Boer, W., Garbeva, P. V. (2015). Volatiles in interspecific bacterial interactions. Frontiers in Microbiology 6,2015.01412.doi:10.3389/fmicb.2015.01412 


\section{Abstract}

The importance of volatile organic compounds for functioning of microbes is receiving increased research attention. However, to date very little is known on how interspecific bacterial interactions effect volatiles production as most studies have been focused on volatiles produced by monocultures of well described bacterial genera. In this study we aimed to understand how interspecific bacterial interactions affect the composition, production and activity of volatiles. Four phylogenetically different bacterial species namely: Chryseobacterium, Dyella, Janthinobacterium and Tsukamurella were selected. Earlier results had shown that pairwise combinations of these bacteria induced antimicrobial activity in agar media whereas this was not the case for monocultures. In the current study, we examined if these observations were also reflected by the production of antimicrobial volatiles. Thus, the identity and antimicrobial activity of volatiles produced by the bacteria were determined in monoculture as well in pairwise combinations. Antimicrobial activity of the volatiles was assessed against fungal, oomycetal and bacterial model organisms.

Our results revealed that interspecific bacterial interactions affected volatiles blend composition. Fungi and oomycetes showed high sensitivity to bacterial volatiles whereas the effect of volatiles on bacteria varied between no effects, growth inhibition to growth promotion depending on the volatile blend composition. In total 35 volatile compounds were detected most of which were sulfur-containing compounds. Two commonly produced sulfurcontaining volatile compounds (dimethyl disulfide and dimethyl trisulfide) were tested for their effect on three target bacteria.

Here we display the importance of interspecific interactions on bacterial volatiles production and their antimicrobial activities. 


\section{Introduction}

Soil bacteria produce an astounding array of secondary metabolites. Gaseous secondary metabolites, commonly known as volatile organic compounds (VOCs) are small molecules (<300 Da) belonging to different chemical classes that can evaporate and diffuse easily through air- and waterfilled pores (Schulz and Dickschat, 2007;Penuelas et al., 2014). These physiochemical properties make volatiles ideal metabolites for communication and antagonistic interactions between soil microorganisms living at a certain distance from each other. Indeed, recent studies indicate that soil microorganisms can employ volatile compounds as info-chemicals, growth stimulants, growth inhibitors and inhibitors of quorum-sensing (Kai et al., 2009;Chernin et al., 2011;Effmert et al., 2012;Kim et al., 2013). Furthermore, rhizosphere bacteria emit volatiles that can promote plant growth and elicit induced systemic resistance (ISR) and induced systemic tolerance (IST) in plants (Ryu et al., 2003;Ryu et al., 2004). However, the role of volatiles in competitive interactions between soil bacteria is so far poorly understood.

In the past few years the research on volatiles emitted by bacteria received increased attention from a more applied point of view as these compounds have intriguing properties which are of great interest for agriculture (pathogen suppression), food preparation (aroma) and cosmetics industry (perfume odors) (Krings and Berger, 1998; Wheatley, 2002;Beshkova et al., 2003;Schwab et al., 2008;Deetae et al., 2009;Effmert et al., 2012;Kanchiswamy et al., 2015).

Bacterial volatiles belong to different chemical classes like alkenes, alcohols, ketones, terpenes, benzenoids, pyrazines, acids and esters. However, the composition of emitted volatiles (volatile blend composition) may vary with cultivation conditions, in particular with respect to the substrate composition of the growth media (Cleason, 2006;Blom et al., 2011;Groenhagen et al., 2013;Garbeva et al., 2014a). Other factors known to influence volatile production are microbial physiological state, oxygen availability, moisture, temperature and $\mathrm{pH}$ (Bjurman, 2007;Insam and Seewald, 2010;Romoli et al., 2014).

The technical developments that have been made in recent years in the field of mass spectrometry have led to the improvement of volatile compounds detection. The details of these developments have recently been summarized 
by Carter (Carter, 2014). However, the main challenge in volatolomics is the ability to identify and quantify the entire set of emitted volatiles. The detected volatile blends are mostly quite complex and make the identification of biologically relevant volatiles a demanding and challenging task (Farag et al., 2012; Tait et al., 2014).

To date more than over 1000 microbial volatiles are reported and described in a special database for microbial volatile organic compounds called mVOC (http://bioinformatics.charite.de/mvoc/) (Lemfack et al., 2014). Nevertheless, this number is rather low compared to the high diversity of bacterial taxa in soil, suggesting a big underestimation of the actual real number of microbial volatiles (Kai et al., 2009;Lemfack et al., 2014). Moreover, most of the studies on microbial volatile detection have dealt with monocultures of already welldescribed bacterial genera. Thus, very little is known on how interspecific interactions affect the volatile production. The investigation of volatiles production in more complex communities is of great interest since it could help to reveal the ecological role of these compounds. In the last years several independent studies reported that the production of secondary metabolites by soil bacteria can be influenced by interactions with microorganisms in their vicinity (Garbeva et al., 2011b;Traxler et al., 2013;Tyc et al., 2014). A highthroughput screening performed recently in our lab revealed that interactions between soil bacterial species have major effects in both directions: induction and suppression of antimicrobial activity (Tyc et al., 2014).

In this study we aimed to understand how interspecific bacterial interactions affect the emission of volatiles and their activity. For this we selected four strains belonging to different bacteria species that have been isolated from the soil bacterial community associated with sand sedge (Carex arenaria L.) namely Chryseobacterium sp. AD48, Dyella sp. AD56, Janthinobacterium sp. AD8o and Tsukamurella sp. AD1o6 (Tyc et al., 2014). In an earlier screening it was observed that these bacteria showed induced antimicrobial activity during interactions but not in monocultures. In the current study, it was examined if these observations were also reflected by the volatiles emission. To this end the effects of volatiles on growth of fungal, oomycetal and bacterial model organisms produced by the bacteria in monocultures as well in pairwise combinations were tested. Our overall hypothesis is that the blend composition volatiles produced during interactions differs from that of monocultures and consequently has different effect on model target organisms.

64 


\section{Materials and methods}

\section{Bacteria \& culture conditions}

The bacterial isolates applied in this work were selected based on a previous observations of antimicrobial activity triggered by interspecific interactions (Tyc et al., 2014). Four bacterial isolates were used: Chryseobacterium sp. AD48 (Class: Flavobacteriia) GenBank: KJ685263, Dyella sp. AD56 (Class: Gammaproteobacteria) GenBank: KJ685269, Janthinobacterium sp. AD8o (Class: Betaproteobacteria) GenBank: KJ685292 and Tsukamurella sp. AD1o6 (Class: Actinobacteria) GenBank: KJ685317. The bacterial isolates were precultured from $-80{ }^{\circ} \mathrm{C}$ glycerol stocks on $1 / 10^{\text {th }}$ TSBA $\left(5.0 \mathrm{gL}^{-1} \mathrm{NaCl}\right.$, $1.0 \mathrm{gL}^{-1}$ $\mathrm{KH}_{2} \mathrm{PO}_{4} ; 3 \mathrm{gL}^{-1}$ Oxoid Tryptic Soy Broth; $20 \mathrm{gL}^{-1}$ Merck Agar, pH 6.5) (Garbeva and de Boer, 2009) and incubated for three days at $24{ }^{\circ} \mathrm{C}$ before starting the experiments.

To test the effect of bacterial volatile compounds on bacterial growth and colony morphology three indicator bacteria were used: E. coli WA321, S. aureus $533 \mathrm{R}_{4}$ (Meyer and Schleifer, 1978;Tyc et al., 2014) and S. marcescens P87 (Garbeva et al., 2014b). All three indicator bacteria were pre-cultured from $-80{ }^{\circ} \mathrm{C}$ glycerol stocks either on LB-A media (LB-Medium Lennox, Carl Roth GmbH + Co. KG, Netherlands, art.no. X964.2, 20 gL $^{-1}$ Merck Agar) (E. coli $\mathrm{WA}_{321}$ and $S$. aureus $533 \mathrm{R}_{4}$ ) (Sambrook and Russell, 2001) or on $1 / 10^{\text {th }}$ TSBA (S. marcescens $\mathrm{P} 87$ ). The indicator organisms E. coli and S. aureus were incubated overnight at $37{ }^{\circ} \mathrm{C}$ prior application, S. marcescens $\mathrm{P} 87$ was incubated at $24{ }^{\circ} \mathrm{C}$ for four days prior usage. All bacterial isolates used in this study are listed in Table 3. 1.

\section{Cultures and growth conditions of fungi and oomycetes}

The fungi Rhizoctonia solani AG2.2IIIB and Fusarium culmorum PV and the oomycete Pythium ultimum P17 were used in this study (Garbeva et al., 2014b). The fungi and oomycete were pre-cultured on $1 / 5^{\text {th }}$ Potato Dextrose Agar (PDA) (29 gL ${ }^{-1}$ Oxoid CM 139) (Fiddaman and Rossall, 1993) and incubated at $24{ }^{\circ} \mathrm{C}$ for 7 days prior usage. All fungal and oomycetal organisms are listed in Table 3.1. 
Table3. 1: Bacterial, fungal and oomycetal organisms used in this study.

\begin{tabular}{|c|c|c|c|c|}
\hline Strain & Phylum/class & Genbank & Reference & Function \\
\hline \multicolumn{5}{|c|}{ volatile producing bacteria tested } \\
\hline Chryseobacterium sp. $\mathrm{AD}_{4} 8$ & Flavobacteriia & $\mathrm{KJ} 685263$ & Tyc et al. 2014 & \multirow{4}{*}{ used for volatile analysis } \\
\hline Dyella sp. AD56 & $\gamma$-proteobacteria & KJ685269 & Tyc et al. 2014 & \\
\hline Janthinobacterium sp. AD8o & $\beta$-proteobacteria & KJ685292 & Tyc et al. 2014 & \\
\hline Tsukamurella sp. ADı66 & Actinobacteria & KJ685317 & Tyc et al. 2014 & \\
\hline \multicolumn{5}{|c|}{ fungal / oomycetal test organisms } \\
\hline Rhizoctonia solani AG2.2IIIB & Basidiomycota & KT124637 & Garbeva et al. 2011 & \multirow{3}{*}{$\begin{array}{l}\text { eukaryotic model } \\
\text { organisms for growth } \\
\text { inhibition }\end{array}$} \\
\hline Pythium ultimum $\mathrm{P}_{17}$ & Oomycete & $\mathrm{KT}_{124638}$ & Garbeva et al. 2014 & \\
\hline Fusarium culmorum PV & Ascomycota & - & Garbeva et al. 2014 & \\
\hline \multicolumn{5}{|l|}{ bacterial test organisms } \\
\hline Serratia marcescens $\mathrm{P} 87$ & $\gamma$-proteobacteria & - & Garbeva et al. 2014 & \multirow{3}{*}{$\begin{array}{l}\text { bacterial model } \\
\text { organisms for growth } \\
\text { inhibition and colony } \\
\text { morphology changes }\end{array}$} \\
\hline $\begin{array}{l}\text { Escherichia coli WA321 } \\
\text { DSMZ } 4509\end{array}$ & $\gamma$-proteobacteria & - & Tyc et al. 2014 & \\
\hline $\begin{array}{l}\text { Staphylococcus aureus } 533 \mathrm{R}_{4} \\
\text { Serovar } 3 \text { DSMZ } 20231\end{array}$ & Firmicutes & LN681573 & Meyer et al. 1978 & \\
\hline
\end{tabular}

\section{Experimental treatments}

Ten different treatments were performed in triplicates. These treatments were: monoculture 1 (Chryseobacterium sp. AD 48), monoculture 2 (Tsukamurella sp. ADio6), monoculture 3 (Dyella sp. AD56), monoculture 4 (Janthinobacterium sp. AD8o) and pairwise interaction of the isolates: interaction 1 (Chryseobacterium sp. AD48 + Tsukamurella ADio6), interaction 2 (Dyella sp. AD 56 + Janthinobacterium sp. AD8o), Control 1 (glass Petri dish with TSBA media without inoculated bacteria, as background control in GC/MS measurement), Control 2 (two compartment Petri dish inoculated with model organisms without exposure to bacterial volatiles), Control 3 (top bottom Petri dish inoculated with fungal/oomycetal model organisms without exposure to bacterial volatile compounds). Control 4 (two compartment Petri dish inoculated with model organisms without exposure to the tested pure volatile compounds). The effect of the produced volatiles was tested on fungal, oomycetal and bacterial growth via determination of hypha biomass or growth inhibition assays. For the inoculation of the experiments a single colony of each test isolate was picked from a plate and inoculated in $20 \mathrm{~mL}$ 1/10 ${ }^{\text {th }}$ TSB (5.0 $\mathrm{gL}^{-1} \mathrm{NaCl}$, $1.0 \mathrm{gL}^{-1} \mathrm{KH}_{2} \mathrm{PO}_{4} ; 3 \mathrm{gL}^{-1}$ Oxoid Tryptic Soy Broth) and incubated overnight at $24{ }^{\circ} \mathrm{C}, 220 \mathrm{rpm}$. On the next day the $\mathrm{OD}_{60}$ of each isolate was measured on a GENESYS $^{\mathrm{TM}} 20$ spectrophotometer (Thermoscientific, Netherlands, Cat\# 4001-ooo) and a inoculation suspension 
for each treatment was prepared in $20 \mathrm{~mL}$ of $10 \mathrm{mM}$ P-Buffer ( $\mathrm{pH} 6.5$ ) containing bacterial cells in a concentration of $\sim 1 \times 10^{\wedge} 5 \mathrm{CFU} / \mathrm{mL}$.

\section{Volatile trapping}

Next to the inhibition experiments, bacterial volatiles emitted in monocultures and pairwise combinations were trapped and analyzed. For trapping of volatile organic compounds emitted by bacteria a volume of $100 \mu \mathrm{l}$ of inoculation suspension was spread on $1 / 10^{\text {th }}$ TSBA $(20 \mathrm{~mL})$ in glass Petri dishes designed for headspace volatile trapping (Garbeva et al., 2014b). The Petri dishes were closed by a lid with an outlet connected to a steel trap containing $150 \mathrm{mg}$ Tenax TA and $150 \mathrm{mg}$ Carbopack B (Markes International Ltd., Llantrisant, UK) (Figure S3.1). All treatments were inoculated in triplicate. The volatiles were collected after 48 and 72 hrs. of incubation and the Tenax steel traps were stored at $4{ }^{\circ} \mathrm{C}$ until GC-Q-TOF analysis.

\section{GC-Q-TOF analysis}

The trapped volatile organic compounds were desorbed from the traps using an automated thermodesorption unit (Unity TD-10o, Markes International Ltd., Llantrisant, UK) at $210^{\circ} \mathrm{C}$ for $12 \mathrm{~min}$ (He flow $50 \mathrm{~mL} / \mathrm{min}$ ) and trapped on a cold trap at $-10{ }^{\circ} \mathrm{C}$. The trapped volatiles were introduced into the GCQTOF (model Agilent 789oB GC and the Agilent 7200A QTOF, Santa Clara, USA) by heating the cold trap for $3 \mathrm{~min}$ to $280{ }^{\circ} \mathrm{C}$. Split ratio was set to 1:10, and the column used was a $30 \times 0.25 \mathrm{~mm}$ ID RXI-5MS, film thickness $0.25 \mu \mathrm{m}$ (Restek 13424-6850, Bellefonte, PA, USA). Temperature program used was as follows: $39{ }^{\circ} \mathrm{C}$ for $2 \mathrm{~min}$, from $39{ }^{\circ} \mathrm{C}$ to $95{ }^{\circ} \mathrm{C}$ at $3.5{ }^{\circ} \mathrm{C} / \mathrm{min}$, then to $165^{\circ} \mathrm{C}$ at $6{ }^{\circ} \mathrm{C} / \mathrm{min}$, to $250^{\circ} \mathrm{C}$ at $15{ }^{\circ} \mathrm{C} / \mathrm{min}$ and finally to $300{ }^{\circ} \mathrm{C}$ at $40{ }^{\circ} \mathrm{C} / \mathrm{min}$, hold 20 min. The MS operating at $70 \mathrm{eV}$ in EI mode detected the VOCs. Mass spectra were acquired in full-scan-mode (30-40oAMU, 4 scans/s). Mass-spectra's were extracted with MassHunter Qualitative Analysis Software V B.o6.oo Build 6.o.633.o (Agilent Technologies, Santa Clara, USA) using the GC-Q-TOF qualitative analysis module. The obtained mass spectra's were exported as mzData files for further processing in MZmine V2.14.2. The files were imported to MZmine V2.14.2 (Copyright (c) 2005-2012 MZmine Development Team) (Katajamaa et al., 2006;Pluskal et al., 2010) and compounds were identified via their mass spectra using deconvolution function (LocalMaximum algorithm) in combination with two mass-spectral-libraries: NIST 2014 V2.20 (National Institute of Standards and Technology, USA, http://www.nist.gov) and Wiley $7^{\text {th }}$ edition spectral libraries and by their linear retention indexes (LRI). The LRI values were calculated using an alkane 
calibration mix before the measurements in combination with AMDIS 2.72 (National Institute of Standards and Technology, USA). The calculated LRI were compared with those found in the NIST and in the in-house NIOO LRI database. After deconvolution and mass identification peak lists containing the mass features of each treatment (MZ-value/Retention time and the peak intensity) were created and exported as CSV files for statistical processing. The whole volatolomic workflow is shown in Figure $\mathbf{S 3 . 2}$.

\section{Bioassay for testing the effect of bacterial volatiles on fungal and oomycete growth}

To test the effect of the emitted bacterial volatiles on fungal/oomycete growth the hyphal extension and biomass were measured. The assays were performed in Petri dishes containing top and bottom growth areas (Figure S3.3). At the bottom of the Petri dish, $100 \mu \mathrm{l}$ of bacterial suspensions in $10 \mathrm{mM}$ phosphate buffer ( $\mathrm{pH}$ 6.5) containing $\sim 1 \times 10^{\wedge} 5 \mathrm{CFU} / \mathrm{mL}$ were spread on $20 \mathrm{~mL} 1 / 10^{\text {th }}$ Tryptic Soy Broth Agar (TSBA). At the lid of the Petri dish $12.5 \mathrm{~mL}$ of wateragar medium (WA) (20 $\mathrm{gL}^{-1}$ MERCK agar) was added and inoculated in the middle with a 6-mm-diameter PDA agar plug containing fungal $(R$. solani, F. culmorum) or oomycete (P. ultimum) hyphae. The plates were sealed with two layers of parafilm and incubated at $24{ }^{\circ} \mathrm{C}$ for five days. In this way the tested fungi were exposed (without direct physical contact) to the volatiles produced by the bacteria in the bottom compartment. On the fifth day the extension of the hyphae was measured in 4 evenly spaced directions and compared to the hyphae extension in the control plates (fungi exposed to $1 / 10^{\text {th }}$ TSBA growth medium without bacteria).

\section{Determination of fungal and oomycetal biomass}

Fungal biomass was determined as described by Garbeva et al. (2014). The whole growth area in the lids containing water agar and fungal hyphae was cut in $\sim 2 \mathrm{~cm}^{2}$ pieces and transferred to a glass beaker containing $100 \mathrm{~mL}$ of sterile demi-water $\left(\mathrm{H}_{2} \mathrm{O}\right)$. The agar was melted for $\sim 2.5 \mathrm{~min}$. in a microwave oven (temperature increased to about $100{ }^{\circ} \mathrm{C}$ ). The melted agar containing the hyphae was filtered over a tea strainer and the remaining hyphae were rinsed with about $150-200 \mathrm{~mL}$ of hot water $\left(\sim 8 \mathrm{o}^{\circ} \mathrm{C}\right)$. The hyphae were picked with tweezers from the tea strainer and transferred to a micro centrifuge tube and stored at $-20{ }^{\circ} \mathrm{C}$ until analysis. For determination of fungal/oomycete biomass the frozen hyphae were transferred to a glass tube with lids with small holes and subjected to freeze-drying for $48 \mathrm{~h}$ (Labconco Freezone 12 with Labcanco Clear Drying Chamber nr.78670oo). The samples were stored in an exsiccator 68 
with dried silica gel for $3 \mathrm{~h}$ (Silica Gel Orange, 2-5 mm, indicator, Roth, art.nr.Po77.2) prior weighing the dry biomass.

\section{Bioassay for testing the effect of bacterial volatiles on growth and colony morphology of target bacteria}

The assays were performed in two-compartment Petri dishes (Greiner bio-one B.V., Alphen a/d Rijn, The Netherlands, Cat\# 635102) containing two separated compartments (Figure $\mathbf{S} \mathbf{3}$ ). In such way the growth response of target bacteria to volatile producing bacteria could be determined without direct physical contacts. One compartment was supplemented with $12.5 \mathrm{~mL}$ TSBA and contained the volatile producing bacteria either in monoculture or in pairwise interactions. The second compartment contained the indicator bacteria and was supplemented either with $12.5 \mathrm{~mL}$ LBA (E. coli WA321, $S$. aureus 533R4) or with $12.5 \mathrm{~mL}$ TSBA (S. marcescens P87). The compartment for the volatile producing bacteria was inoculated with $100 \mu$ bacterial suspensions master mix of monocultures or pairwise interactions prepared with $20 \mathrm{~mL}$ of $10 \mathrm{mM}$ phosphate buffer ( $\mathrm{pH} 6.5$ ) containing $\sim 1 \times 10^{\wedge} 5 \mathrm{CFU} / \mathrm{mL}$. The compartment for the indicator organisms was inoculated with four droplets $(5 \mu \mathrm{L})$ of each indicator bacteria. The droplets of the indicator bacteria were placed in a distance of $2 \mathrm{~cm}$ to each other and contained $1 \mathrm{x}$ $10^{\wedge} 5,1 \times 10^{\wedge} 4,1 \times 10^{\wedge} 3$ and $1 \times 10^{\wedge} 2 \mathrm{CFU} / \mathrm{mL}$ of either $E$. coli WA321, S. aureus $533 \mathrm{R}_{4}$ or $S$. marcescens $\mathrm{P} 87$ (Figure S3.4). As controls the first compartment of the Petri dish was kept empty. After four days of incubation at $24{ }^{\circ} \mathrm{C}$ the plates were examined and digital photographs were taken. The digital images were analyzed using the AXIO VISION v4.8 imaging Software (Carl Zeiss Imaging Solutions $\mathrm{GmbH}$, Germany) for enumeration and surface-area determination (in $\operatorname{pixel}^{\wedge} \mathbf{2}$ ) of the bacterial colonies. All treatments were performed in triplicate.

\section{Test of pure volatile compounds on bacterial growth and colony morphology}

The effect on growth, colony morphology and pigmentation by pure dimethyl disulfide $\left(\mathrm{CH}_{3} \mathrm{~S}_{2} \mathrm{CH}_{3}\right)$, dimethyl trisulfide $\left(\mathrm{CH}_{3} \mathrm{~S}_{3} \mathrm{CH}_{3}\right)$ and the mixture of both compounds was tested on E. coli WA321, S. aureus $533 \mathrm{R} 4$ and $S$. marcescens P87. The assays were performed in two-compartment Petri dishes (Greiner bio-one B.V., Alphen a/d Rijn, The Netherlands, Cat\# 635102). Both compartments were supplemented with either $12.5 \mathrm{~mL}$ LB-A (assay performed with E. coli WA321 and S. aureus 533R4) or with $12.5 \mathrm{~mL}$ TSBA (assay performed with $S$. marcescens $\mathrm{P} 87$ ). In one compartment a filter paper with a 
diameter of $\sim 5,5 \mathrm{~mm}$ (Whatman ${ }^{\mathrm{TM}}$ filter paper Cat\# 1003-150, $6 \mu \mathrm{m}$ pore size) was placed on the agar surface in the middle of the compartment. Stock solutions with a concentration of $10 \mu \mathrm{M}, 1 \mu \mathrm{M}$ and $0.1 \mu \mathrm{M}$ of the pure volatile compounds (dimethyl disulfide or dimethyl trisulfide) and the mixture of both compounds (dimethyl disulfide + dimethyl trisulfide) were prepared by serial dilution of the pure compounds in Methanol (LiChrosolv", Index-No: 603-oo1-oo-X, Merck, Darmstadt, Germany). For the test a volume of $5 \mu \mathrm{lof}$ each of the pure volatile stock solutions was added directly onto the filter paper resulting in a final concentration of $50 \mu \mathrm{M}, 5 \mu \mathrm{M}$ and $0.5 \mu \mathrm{M}$, respectively. The other compartment was inoculated with the target bacteria E. coli WA321, S. aureus $533 \mathrm{R}_{4}$ or $S$. marcescens $\mathrm{P} 87$ by inoculating four spots in a distance of $2 \mathrm{~cm}$ from each other containing $1 \times 10^{\wedge} 5,1 \times 10^{\wedge} 4,1 \times 10^{\wedge} 3$ and $1 \times 10^{\wedge_{2}} \mathrm{CFU} / \mathrm{mL}$ (Figure S3.4 $_{3}$ ). As controls bacteria exposed to filter papers with no added volatile compounds were applied. The Petri dishes were sealed with a double layer of parafilm and incubated for four days at $24{ }^{\circ} \mathrm{C}$. After incubation digital photographs were taken and the effect on colony growth, colony morphology and pigment production (prodigiosin) in $S$. marcescens P87 was examined. All digital images were analyzed using the AXIO VISION v4.8 imaging Software (Carl Zeiss Imaging Solutions GmbH, Germany) for enumeration and surface-area determination (in $\operatorname{pixel}^{\wedge} 2$ ) of the bacterial colonies. All treatments were performed in triplicate.

\section{Statistical analysis}

Statistical analysis on volatolomic data was performed using the statistical analysis module of MetaboAnalyst V3.o, www.metaboanalyst.ca (Xia et al., 2012;Xia et al., 2015). Prior to statistical analysis data normalization was performed via log-transformation. To identify significant abundant mass features ONE-WAY-ANOVA with post-hoc Tukey test (HSD- test) was performed between the data sets. To identify important mass features in the samples PLS-D analysis was performed. Mass features were considered to be statistical relevant if $\mathrm{p}$ - values were $\leq 0.05$. Statistical relevant mass features were further used for the compound identification. Statistical analyses on fungal dry biomass and bacterial colony sizes were performed with IBM SPSS Statistics 23 (IBM, Somers, NY, USA) using one-way ANOVA and post-hoc Tukey test between the data sets. The $5 \%$ level was taken as threshold for significance between control and volatile treatments. 


\section{Determination of $\mathrm{HCN}, \mathrm{NH}_{3}$ emission and $\mathrm{pH}$ values in the agar}

All bacterial strains used in this study were tested for the emission of ammonia and $\mathrm{HCN}$ as well as for the ability to change the $\mathrm{pH}$ - value of the growth medium where the target organisms were inoculated. For these tests the bacteria were inoculated in two-compartment petri dishes (start density $\left.\sim 1 \times 10^{\wedge} 5 \mathrm{CFU} / \mathrm{mL}\right)$ on $12.5 \mathrm{~mL} 1 / 10^{\text {th }}$ TSBA. The second compartment was supplemented with $12.5 \mathrm{~mL}$ water-agar (WA). After 4 days of growth the HCN and ammonia emission as well the $\mathrm{pH}$-value of the target organism growth medium (WA) was determined. To test for the presence of Hydrocyanic acid the gaseous content of the petri dish headspace was sucked through a Hydrocyanic acid test tube (Dräger Safety AG \& CO. KGaA, Lübeck, Germany, order number: $\mathrm{CH}_{25701)}$ using the Dräger accuro $^{\circledast}$ gas detection pump (Dräger Safety AG \& CO. KGaA, Lübeck, Germany).

Presence of Hydrocyanic acid was determined by colour change of the test tube (formation of a red reaction product) (Figure $\mathbf{S}_{\mathbf{3} \cdot \mathbf{5}}$ ).

The $\mathrm{pH}$ of the target organism growth medium (WA) exposed to bacterial volatiles was determined by slightly pressing a $\mathrm{pH}$ test-strip VWR PROLABO dosatest $^{\circledast}$ (VWR international, Cat\# 35309.606UK) for 30 seconds into the agar surface. The $\mathrm{pH}$ values were determined by colour change of the test strip and compared to the colour scale on the package (Figure S3.6). $^{3}$.

The ammonia concentration was determined using the MQuant ${ }^{\mathrm{TM}}$ ammonium test kit (Merck, Darmstadt, Germany, Cat\# 110024) by placing a reaction activated test-strip on the lid of the petri dish directly opposite to the bacterial culture and fixed with tape. The petri dish were closed and sealed with parafilm and incubated for 2 hours at $24{ }^{\circ} \mathrm{C}$. After incubation the presence of ammonium was determined by colour change of the test strip (Figure S3.7). 


\section{Results}

\section{Detected headspace volatile compounds and GC/MS-Q-TOF analysis}

GC/MS-Q-TOF based volatolomic analysis revealed a total number of 35 compounds that were not detected in the non-inoculated controls (Table 3.2). 27 compounds were obtained from the monocultures of Chryseobacterium sp. $\mathrm{AD}_{48}, 15$ compounds were obtained from the monocultures of Tsukamurella sp. AD1o6 and 26 compounds were detected in the interactions between these two bacteria (Table 3.2, Figure 3.1A). For the combinations of Dyella sp. AD56 and Janthinobacterium sp. AD8o we obtained a total number of 18 compounds, whereas 16 compounds were detected in the monoculture of Janthinobacterium sp. AD8o and only 13 compounds in the monoculture of Dyella sp. AD56 (Table 3.2, Figure 3.1B). We were able to tentatively identify 19 volatile organic compounds belonging to seven different chemical classes including alcohols, amines, esters, indole, thiocyanates, thioesters and sulfides. However a vast number of the detected compounds $(n=16)$ could not be assigned with certainty to a volatile organic compound and remained unknown. The most prominent detected headspace volatile organic compounds were sulfur-containing compounds (such as sulfur dioxide, methyl thioacetate, dimethyl sulfoxide, etc.). Two sulfur compounds dimethyl disulfide $\left(\mathrm{C}_{2} \mathrm{H}_{6} \mathrm{~S}_{2}\right)$ and dimethyl trisulfide $\left(\mathrm{C}_{2} \mathrm{H}_{6} \mathrm{~S}_{3}\right)$ were produced by all bacteria (except dimethyl trisulfide which was not detected for Janthinobacterium sp. AD8o).

\section{Effect of interspecific interactions on bacterial volatile blend composition}

Volatolomic analysis on monocultures and pairwise combinations of Chryseobacterium sp. AD48 with Tsukamurella sp. ADio6 revealed that the volatile composition of the monocultures differed from that of the mixtures (Figure 3.1A, Table 3.2). Clear separations between controls, monocultures and pairwise combinations of Chryseobacterium sp. $\mathrm{AD}_{4} 8$ with Tsukamurella sp. ADio6 were obtained in PCA score plots (Figure 3.1A). The volatile composition of the pairwise combinations resembled that of the monocultures of Chryseobacterium sp. $\mathrm{AD}_{4} 8$ (Figure 3.1A, Table 3.2). The indole produced by the monoculture of Chryseobacterium sp. $\mathrm{AD}_{4} 8$ was not detected in the interactions (Table 3.2). 
The analysis on the volatiles emitted by monocultures and pairwise combinations of Dyella sp. AD56 and Janthinobacterium sp. AD8o revealed that the volatile profiles of the monocultures differed from that of the mixtures (Figure 3.1B, Table 3.2). Different PCA score plots were obtained between controls, monocultures and pairwise combinations of Dyella sp. AD56 with Janthinobacterium sp. AD8o (Figure 3.1B). A higher number of volatile compounds were detected in the pairwise combinations of these two bacteria. However the higher number of detected volatiles is most probably due to the combination of the volatile blends of these two bacterial isolates. We did not detect any novel or different volatile compounds which production was triggered during the pairwise interaction of these two bacteria. Interestingly the volatile compound cyclopentene produced by the monocultures of Dyella sp. AD56 and Janthinobacterium sp. AD8o was not detected in the interactions (Table 3. 2).

A

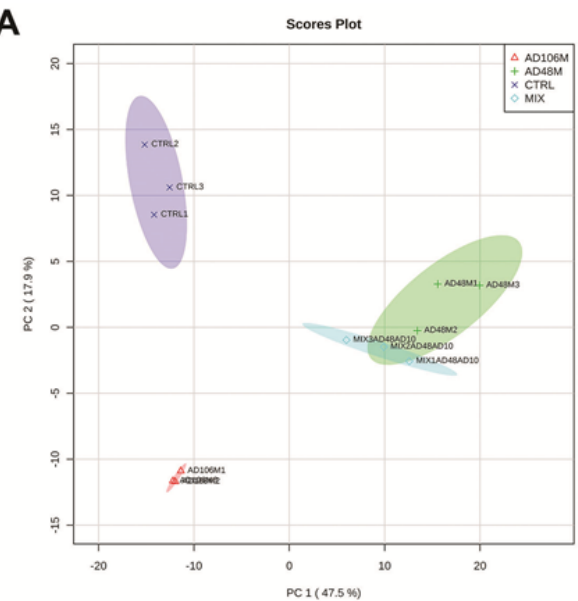

B

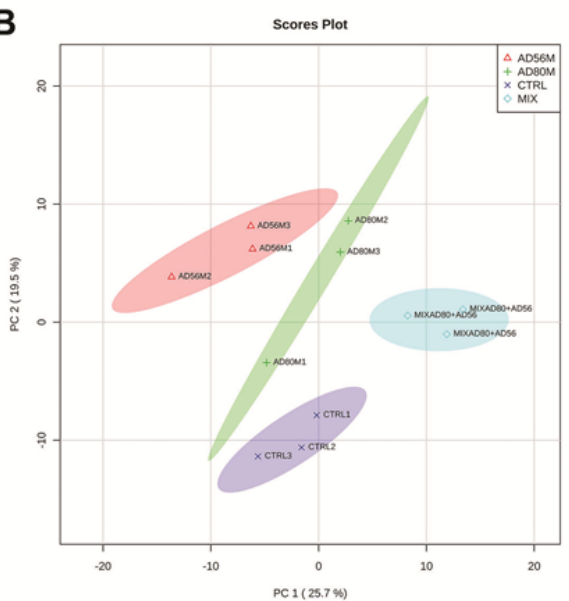

Figure 3.1: PCA 2D- plots of volatiles emitted by monocultures and pairwise combinations of bacteria including confidence intervals (in semi-transparent colors) (A) monocultures and mixtures of Tsukamurella sp. AD1o6 and Chryseobacterium sp. AD48 and (B) monocultures and mixtures of Dyella sp. AD56 and Janthinobacterium sp. AD8o. 
Table 3.2: Tentatively identified volatile organic compounds emitted by four bacterial strains cultivated either in monoculture or in pairwise combination.

\begin{tabular}{|c|c|c|c|c|c|c|c|c|c|}
\hline \multirow[b]{2}{*}{ \# } & \multirow[b]{2}{*}{ Compound name / chemical class } & \multirow[b]{2}{*}{$\mathbf{R T}^{*}$} & \multirow[b]{2}{*}{ ERI** } & \multirow[b]{2}{*}{ Chry } & \multirow[b]{2}{*}{ Tsuk } & \multicolumn{3}{|c|}{ Detected in treatment } & \multirow[b]{2}{*}{$\begin{array}{c}\text { MIX Jant + } \\
\text { Dye }\end{array}$} \\
\hline & & & & & & $\begin{array}{c}\text { MIX Chry + } \\
\text { Tsuk }\end{array}$ & Dye & Jant & \\
\hline 1 & Sulfurdioxide & 2,58 & 521 & $\mathbf{x}$ & & $\bar{x}$ & & $\mathbf{x}$ & $\mathbf{x}$ \\
\hline 2 & Cyclopentene & 2,96 & 551 & $\mathbf{x}$ & & $\mathbf{x}$ & $\mathbf{x}$ & $\mathbf{x}$ & \\
\hline 3 & 2 -Pentene & 3,29 & 575 & & & & $\mathbf{x}$ & & $\mathbf{x}$ \\
\hline 4 & Unknown compound 1 & 3,77 & 612 & $\mathbf{x}$ & $\mathbf{x}$ & $\mathbf{x}$ & $\mathbf{x}$ & $\mathbf{x}$ & $\mathbf{x}$ \\
\hline 5 & Methyl isobutyrate & 4,70 & 682 & & & & $\mathbf{x}$ & & \\
\hline 6 & Methyl thioacetate & 4,94 & 700 & $\mathbf{x}$ & & $\mathbf{x}$ & & $\mathbf{x}$ & $\mathbf{x}$ \\
\hline 7 & Methyl thiocyanate & 5,28 & 713 & & $\mathbf{x}$ & & & $\mathbf{x}$ & $\mathbf{x}$ \\
\hline 8 & 1-Butanol, 3-methyl- & 5,69 & 728 & $\mathbf{x}$ & & $\mathbf{x}$ & & & \\
\hline 9 & Dimethyl disulfide & 6,10 & 744 & $\mathbf{x}$ & $\mathbf{x}$ & $\mathbf{x}$ & $\mathbf{x}$ & $\mathbf{x}$ & $\mathbf{x}$ \\
\hline 10 & Methyl isovalerate & 6,86 & 769 & & & & $\mathbf{x}$ & & \\
\hline 11 & S-methyl propanethioate & 7,45 & 782 & $\mathbf{x}$ & & $\mathbf{x}$ & & $\mathbf{x}$ & $\mathbf{x}$ \\
\hline 12 & 1,3 Dithiethane & 7,64 & 786 & $\mathbf{x}$ & $\mathbf{x}$ & $\mathbf{x}$ & & $\mathbf{x}$ & $\mathbf{x}$ \\
\hline 13 & Dimethyl sulfoxide & 8,46 & 806 & & $\mathbf{x}$ & & & & \\
\hline 14 & 2,4-Dithiapentane & 10,74 & 865 & $\mathbf{x}$ & $\mathbf{x}$ & $\mathbf{x}$ & & $\mathbf{x}$ & $\mathbf{x}$ \\
\hline 15 & Benzaldehyd & 13,72 & 944 & $\mathbf{x}$ & $\mathbf{x}$ & $\mathbf{x}$ & & $\mathbf{x}$ & $\mathbf{x}$ \\
\hline 16 & Dimethyl trisulfide & 14,33 & 960 & $\mathbf{x}$ & $\mathbf{x}$ & $\mathbf{x}$ & $\mathbf{x}$ & & $\mathbf{x}$ \\
\hline 17 & Unknown cycloalkane & 16,86 & 1026 & $\mathbf{x}$ & $\mathbf{x}$ & $\mathbf{x}$ & $\mathbf{x}$ & $\mathbf{x}$ & $\mathbf{x}$ \\
\hline 18 & Unknown branched alkene & 17,39 & 1040 & $\mathbf{x}$ & $\mathbf{x}$ & $\mathbf{x}$ & $\mathbf{x}$ & $\mathbf{x}$ & $\mathbf{x}$ \\
\hline 19 & Unknown sulfur containing compound & 18,09 & 1058 & $\mathbf{x}$ & $\mathbf{x}$ & $\mathbf{x}$ & & & \\
\hline 20 & 1,2,4-Trithiolane & 19,30 & 1090 & $\mathbf{x}$ & $\mathbf{x}$ & $\mathbf{x}$ & & $\mathbf{x}$ & $\mathbf{x}$ \\
\hline 21 & Unknown compound 2 & 19,70 & 1101 & & & & $\mathbf{x}$ & $\mathbf{x}$ & $\mathbf{x}$ \\
\hline 22 & Unknown compound 3 & 19,99 & 1110 & $\mathbf{x}$ & $\mathbf{x}$ & $\mathbf{x}$ & $\mathbf{x}$ & $\mathbf{x}$ & $\mathbf{x}$ \\
\hline 23 & Unknwon compound 4 & 20,63 & 1131 & $\mathbf{x}$ & & $\mathbf{x}$ & & & \\
\hline 24 & Dimethyl tetrasulfide & 23,64 & 1227 & & $\mathbf{x}$ & & & & \\
\hline 25 & Indole & 25,82 & 1298 & $\mathbf{x}$ & & & & & \\
\hline 26 & Butylhydroxytoluene & 30,28 & 1540 & $\mathbf{x}$ & $\mathbf{x}$ & $\mathbf{x}$ & $\mathbf{x}$ & $\mathbf{x}$ & $\mathbf{x}$ \\
\hline 27 & Unknown terpene like compound 1 & 32,84 & 1674 & $\mathbf{x}$ & & $\mathbf{x}$ & & & \\
\hline 28 & Unknown terpene like compound 2 & 33,46 & 1703 & $\mathbf{x}$ & & $\mathbf{x}$ & & & \\
\hline 29 & Unknown tetralin isomer & 33,75 & 1710 & $\mathbf{x}$ & & $\mathbf{x}$ & & & \\
\hline 30 & Unknown aromat isomer & 34,22 & 1721 & $\mathbf{x}$ & & $\mathbf{x}$ & & & \\
\hline 31 & Unknown compound 5 & 34,34 & 1724 & $\mathbf{x}$ & & $\mathbf{x}$ & & & \\
\hline 32 & Unknown di-terpene & 34,78 & 1734 & $\mathbf{x}$ & & $\mathbf{x}$ & & & \\
\hline 33 & Unknown terpene like compound 3 & 35,31 & 1746 & $\mathbf{x}$ & & $\mathbf{x}$ & & & \\
\hline 34 & Unknown compound 6 & 38,73 & 2101 & & & & $\mathbf{x}$ & & $\mathbf{x}$ \\
\hline & Unknown compound 7 & 42,04 & 2360 & $\mathbf{x}$ & & $\mathbf{x}$ & & & \\
\hline \multicolumn{2}{|r|}{ Number of detected compounds (n) } & & & 27 & 15 & 26 & 13 & 16 & 18 \\
\hline
\end{tabular}

Abbreviations:

\# = Compound number, Chry = Chryseobacterium, Dye = Dyella, Jant = Janthinobacterium, Tsuk $=$ Tsukamurella, MIX Chry + Tsuk $=$ pairwise combination of Chryseobacterium + Tsukamurella . MIX Jant + Dye $=$ pairwise combination of Dyella + Janthinobacterium.

$\mathrm{X}=$ detected.

$\mathrm{RT}^{*}=$ Retention time, the RT value stated is the average.

$\mathrm{ERI}^{* *}=$ Experimental retention index value, the RI value stated is the average. 


\section{Effect of bacterial volatiles on fungal and oomycetal growth}

Volatiles produced by all treatments including monocultures and pairwise combinations of the selected bacteria revealed strong growth inhibition of the plant pathogenic fungi and oomycete.

The dry biomass of fungi and oomycete exposed to bacterial volatiles was significantly reduced as compared to the controls without bacterial volatiles (Table 3.3, Figure 3.2, 3.3).

Table 3.3: Effect of bacterial volatiles on fungal and oomycetal biomass production (mg/dry weight of fungal/oomycetal biomass). Data represent mean and standard deviation of three replicates.

\begin{tabular}{|c|c|c|c|}
\hline Treatment & F. culmorum & P. ultimum & R. solani \\
\hline \multicolumn{4}{|l|}{ Monocultures } \\
\hline Chryseobacterium sp. $\mathrm{AD}_{4} 8$ & $1.63 \pm 0.25^{*}$ & $0.83 \pm 0.28^{*}$ & $1.67 \pm 0.75^{*}$ \\
\hline Dyella sp. $\mathrm{AD}_{56}$ & $1.03 \pm 0.55^{*}$ & $1.47 \pm 0.47^{*}$ & $1.1 \pm 0.71^{*}$ \\
\hline Janthinobacterium sp. AD8o & $1.05 \pm 0.77^{*}$ & $0.9 \pm 0.44^{*}$ & $1.1 \pm 0.44^{*}$ \\
\hline Tsukamurella sp. AD1o6 & $2.3 \pm 0.69^{*}$ & $1.47 \pm 0.12^{*}$ & $2.67 \pm 0.47^{*}$ \\
\hline \multicolumn{4}{|l|}{ Interactions } \\
\hline $\begin{array}{l}\text { Chryseobacterium sp. AD } 48+ \\
\text { Tsukamurella sp. AD1o6 }\end{array}$ & $1.73 \pm 0.4^{*}$ & $1.47 \pm 0.25^{*}$ & $2.53 \pm 0.37^{*}$ \\
\hline $\begin{array}{l}\text { Janthinobacterium sp. AD8o + } \\
\text { Dyella sp. AD } 6\end{array}$ & $1.3 \pm 1.27^{*}$ & $0.97 \pm 0.40^{*}$ & $1.23 \pm 0.15^{*}$ \\
\hline Controls & $5 \cdot 97 \pm 2.13$ & $4.42 \pm 0.88$ & $5.47 \pm 1.23$ \\
\hline
\end{tabular}

Asterisk indicates significant differences between the treatments and the respective control (ONE-WAY ANOVA, post-hoc Tukey test $\mathrm{p}<0.05)$. 


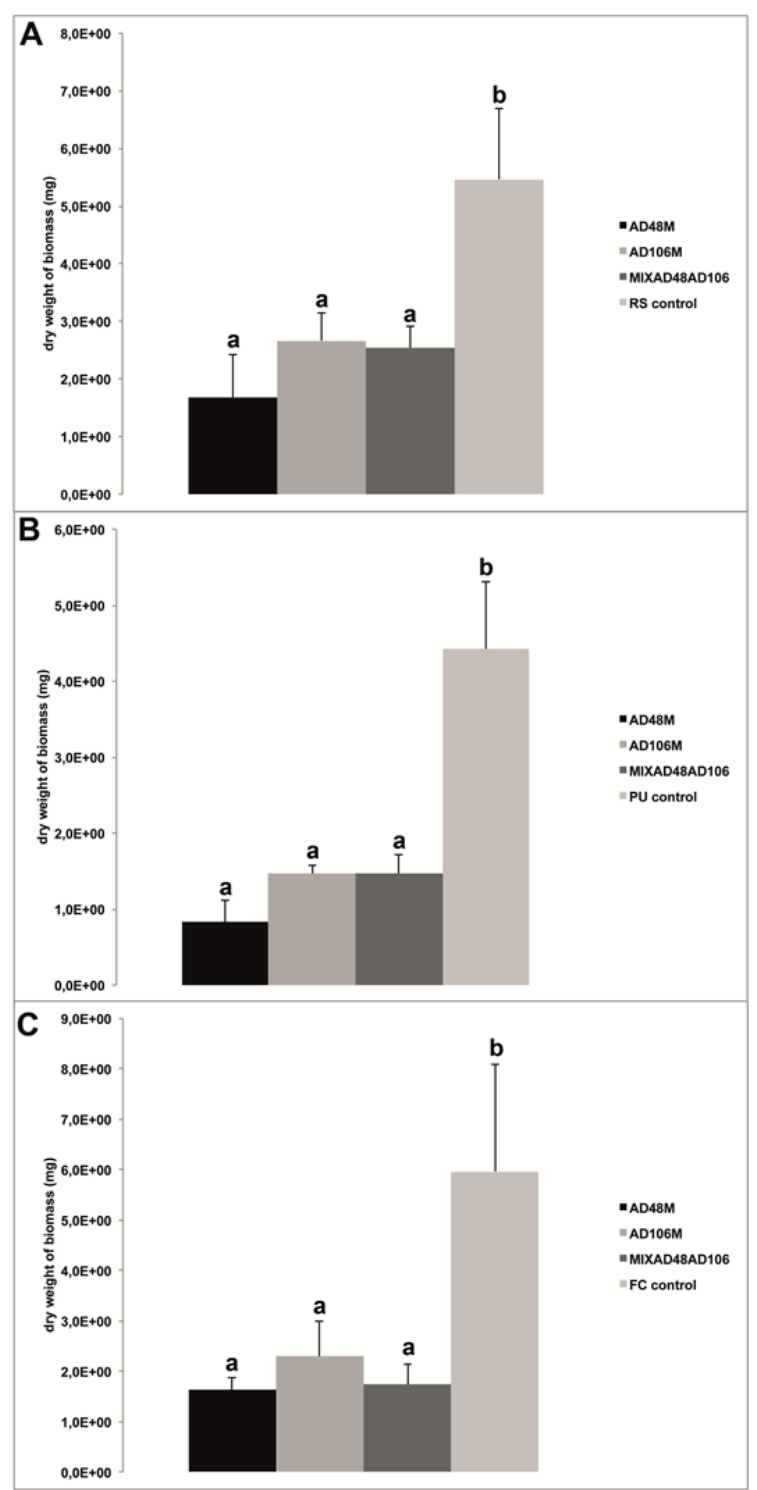

Figure 3.2: Effect of volatiles produced by monocultures and mixtures of Tsukamurella sp. AD1o6 and Chryseobacterium sp. $\mathrm{AD}_{4} 8$ on growth of eukaryotic plant-pathogens. Bars represent the average values for fungal and oomycetal biomass dry weight and error bars represent standard deviation of the mean. (A) Dry weight of $R$. solani (B) Dry weight of P. ultimum (C) Dry weight of F. culmorum. Significant differences between treatments and the control are indicated by different letters (ONE-WAY ANOVA, post-hoc Tukey test $\mathrm{p}<0.05)$. 


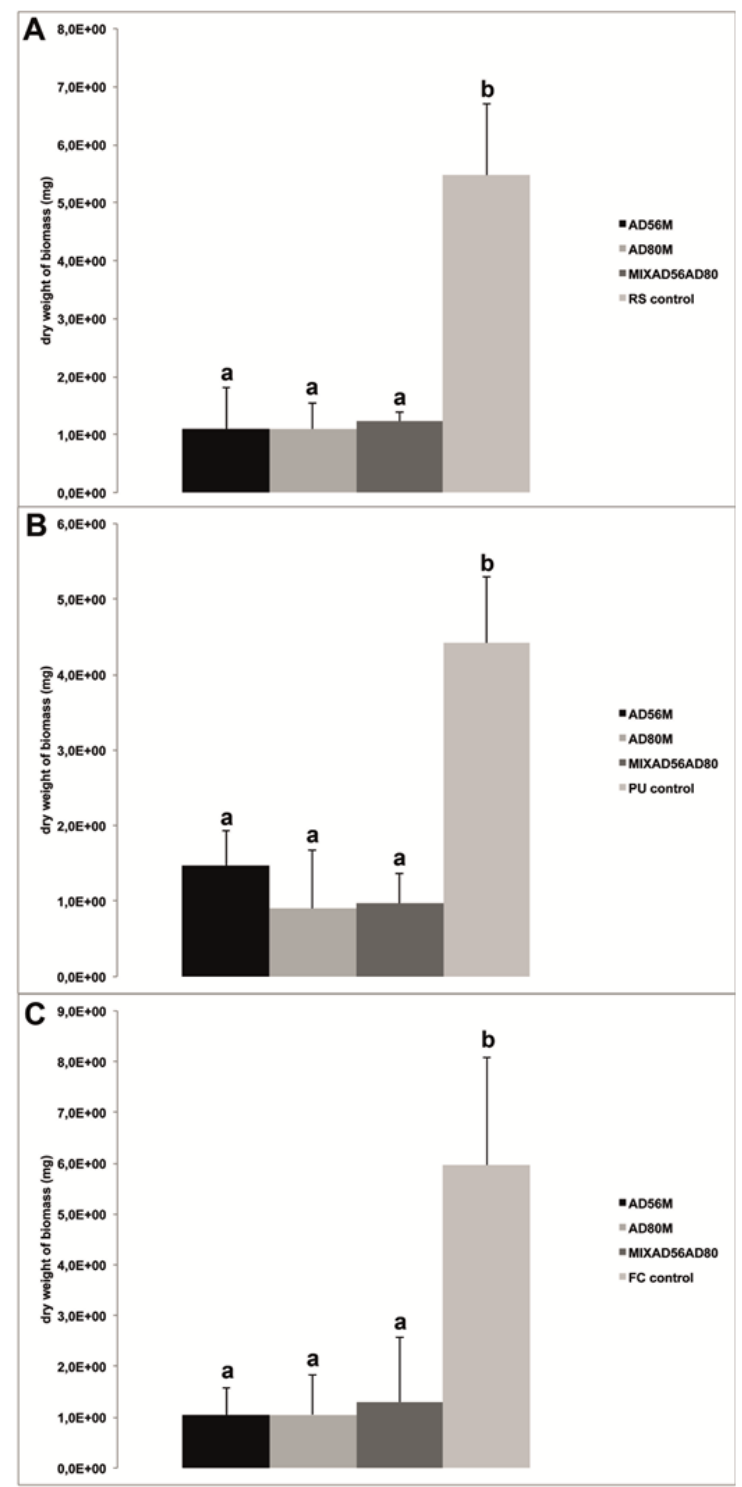

Figure 3.3: Effect of volatiles produced by monocultures and mixtures of volatile emitting Dyella sp. $\mathrm{AD}_{5} 6$ and Janthinobacterium sp. AD8o on growth of eukaryotic plant-pathogens. Bars represent the average values for fungal and oomycetal biomass dry weight and error bars represent standard deviation of the mean. (A) Dry weight of $R$. solani (B) Dry weight of $P$. ultimum (C) Dry weight of $F$. culmorum. Significant differences between treatments and the control are indicated by different letters (ONE-WAY ANOVA, post-hoc Tukey test $\mathrm{p}<0.05)$. 
Effect of bacterial volatiles on the growth and behavior of target bacteria

Volatiles emitted by Chryseobacterium sp. $\mathrm{AD}_{4} 8$ and the mixture of Chryseobacterium sp. $\mathrm{AD}_{4} 8$ and Tsukamurella sp. AD1o6 inhibited the growth of E. coli WA321 significantly as compared to the control (Figure 3.4A). This observation is in agreement with the observed volatolomic profile (Figure 3.1A) which revealed that the volatolomic profile of the mixture is dominated by the volatiles produced by the monoculture of Chryseobacterium sp. $\mathrm{AD}_{4} 8$.

Besides growth inhibition we observed significant growth promotion of S. aureus $533 \mathrm{R} 4$ when exposed to volatiles emitted by the monocultures of Dyella sp. AD56 (Figure 3.4B). Changes in colony morphology of $S$. marcescens $\mathrm{P} 87$ were observed when exposed to volatiles emitted by Chryseobacterium sp. $\mathrm{AD}_{4} 8$ and to volatiles emitted by the mixtures of Dyella sp. AD56 with Janthinobacterium sp. AD8o. The S. marcescens P87 colonies were more circular and round shaped (Figure S3.8). However, no significant effects of bacterial volatiles on the growth of the target bacteria were also observed (Figure S3.9). 


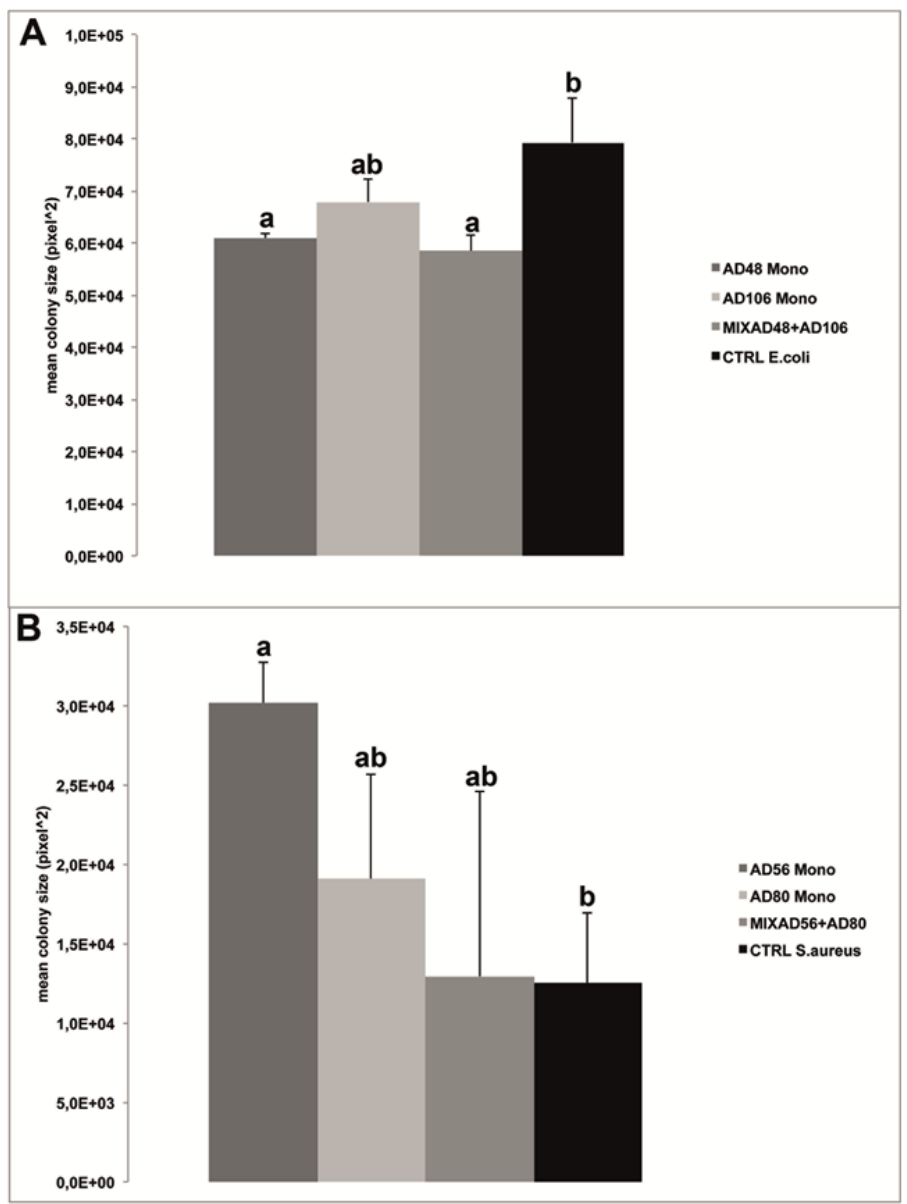

Figure 3.4: Effect of volatiles produced by monocultures and pair-wise combinations of the four selected rhizosphere bacterial strains on average colony size of the target bacteria. (A) Mean colony size of E.coli WA321 exposed to volatile compounds of Chryseobacterium sp. $\mathrm{AD}_{4} 8$ and Tsukamurella sp. AD1o6 and the mixture of both bacteria. (B) Mean colony sizes of S.aureus $533 \mathrm{R}_{4}$ exposed to volatile compounds of Dyella sp. AD56, Janthinobacterium sp. AD8o and the mixture of both bacteria. Significant differences between treatments and the control are indicated by different letters (ONE-WAY ANOVA, post-hoc Tukey test $\mathrm{p}<0.05$ ). Data represented are the mean of three replicates, error bars represent standard deviation of the mean. 
Effect of pure individual volatile compounds on the growth and colony morphology of target bacteria

We applied a two-compartment Petri dish testing system (Figure S3.4) in which the model organisms could grow without direct physical contacts to the tested pure volatile compounds. After four days of growth $S$. marcescens P87 colonies were small and showed a white phenotype when exposed to 50 $\mu \mathrm{M}$ of dimethyl trisulfide, indicating the lack of prodigiosin production (Figure 3.5A). Furthermore we observed significant inhibition of growth of S. marcescens $\mathrm{P} 87$, E. coli WA321 and $S$. aureus $533 \mathrm{R}_{4}$ when exposed to $50 \mu \mathrm{M}$ of dimethyl trisulfide (Figure 3.5A, B; 3.6A, B; 3.7A, B).

Exposure to dimethyl disulfide did not reveal any significant growth inhibiting or changes in colony morphology at all concentrations tested (500 nM, $5 \mu \mathrm{M}$, $50 \mu \mathrm{M})$. The mixture of dimethyl disulfide and dimethyl trisulfide resulted in growth inhibition of $S$. marcescens $\mathrm{P} 87$ and E. coli WA321 at $50 \mu \mathrm{M}$ concentration. However the mixture of these compounds did not affect the pigmentation in S. marcescens $\mathrm{P} 87$.

The two lowest applied concentrations $5 \mu \mathrm{M}$ and o, $5 \mu \mathrm{M}$ of dimethyl trisulfide and dimethyl disulfide and the mixture of both compounds did not reveal any effect on colony morphology or growth of the tested bacteria (Figure 3.5A, B; 3.6A, B; 3.7A, B). 


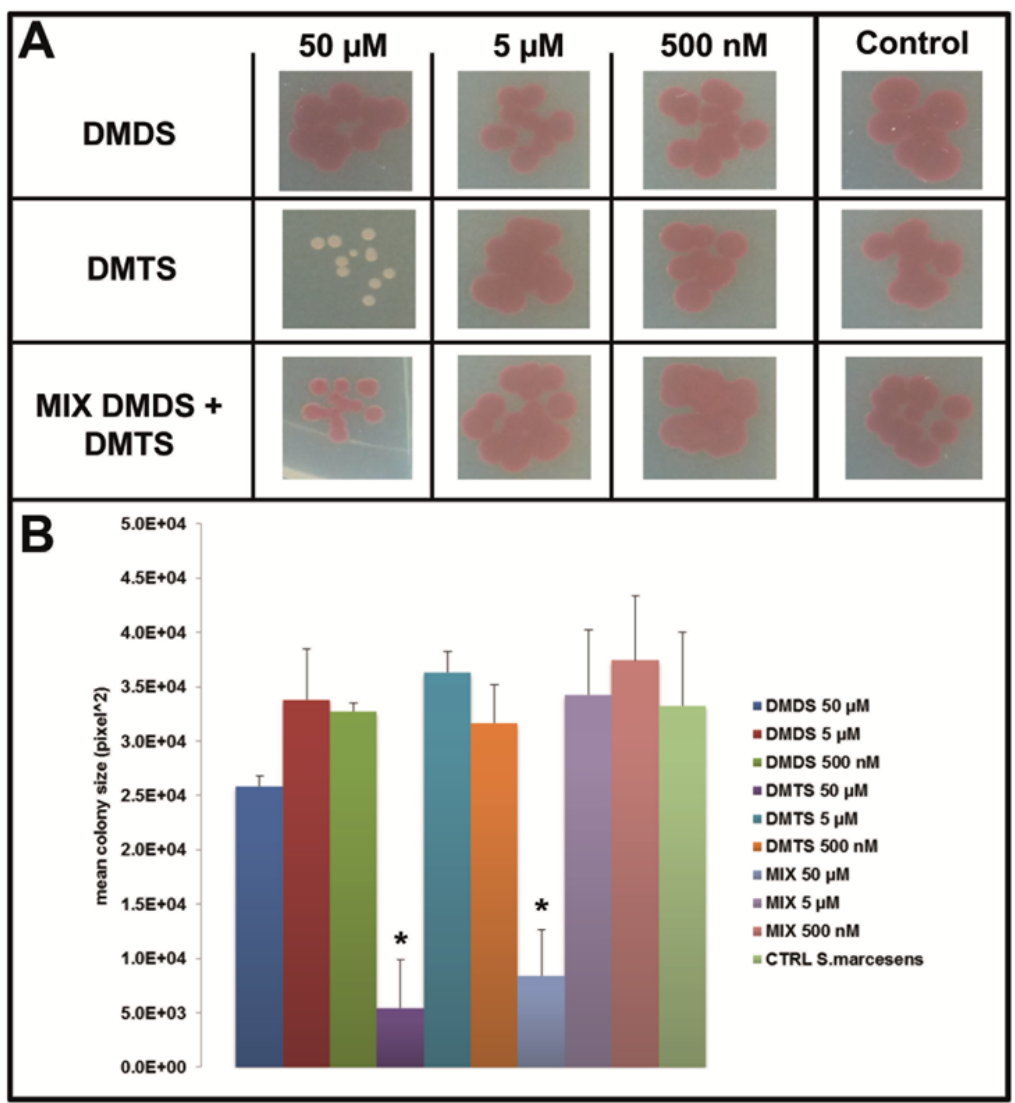

Figure 3.5: Effect of dimethyl disulfide (DMDS), dimethyl trisulfide (DMTS) and the mixture of both volatile compounds (DMDS + DMTS) on colony development of $S$. marcescens (A) colony morphology and growth of S. marcescens $\mathrm{P} 87$ after four days of incubation. The pure volatile compounds were applied in a concentration ranging from $500 \mathrm{nM}$ to $50 \mu \mathrm{M}$. Control S. marcescens $\mathrm{P} 87$ grown without exposure to the compounds. (B) Mean colony sizes of $S$. marcescens $\mathrm{P} 87$ exposed to volatile compounds of dimethyl disulfide (DMDS), dimethyl trisulfide (DMTS) and the mixture of both volatile compounds (DMDS + DMTS). Asterisk indicates significant differences between the treatments and the control (ONE-WAY ANOVA, post-hoc Tukey test $\mathrm{p}<0.05)$. Data represented are the mean of three replicates, error bars represent standard deviation of the mean. 


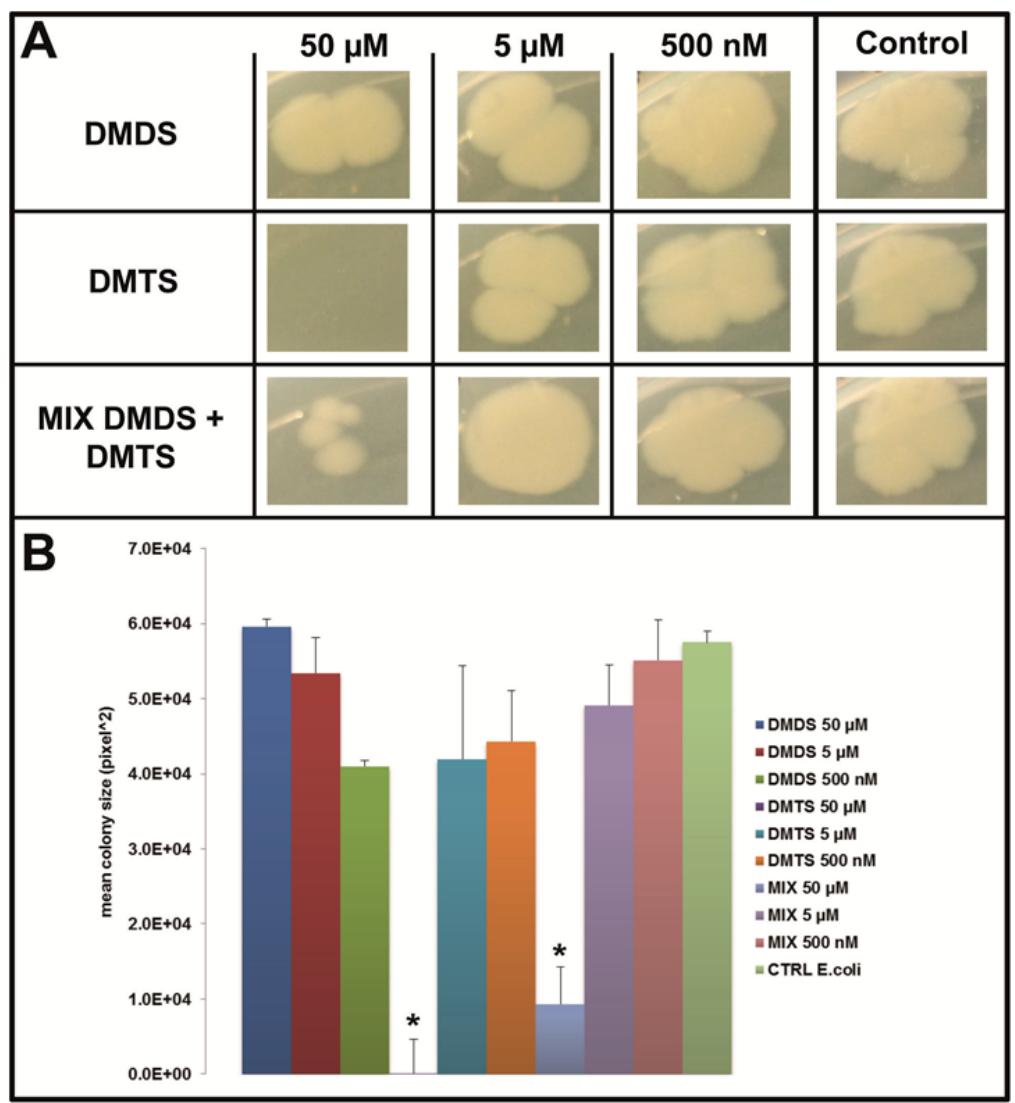

Figure 3.6: Effect of dimethyl disulfide (DMDS), dimethyl trisulfide (DMTS) and the mixture of both volatile compounds (DMDS + DMTS) (A) colony morphology and growth of E. coli WA321 after four days of incubation. The pure volatile compounds were applied in a concentration ranging from $500 \mathrm{nM}$ to $50 \mu \mathrm{M}$. Control E. coli WA321 grown without exposure to the compounds. (B) Mean colony sizes of E. coli WA321 exposed to volatile compounds of dimethyl disulfide (DMDS), dimethyl trisulfide (DMTS) and the mixture of both volatile compounds (DMDS + DMTS). Asterisk indicates significant differences between the treatments and the control (ONE-WAY ANOVA, post-hoc Tukey test $\mathrm{p}<0.05$ ). Data represented are the mean of three replicates, error bars represent standard deviation of the mean. 


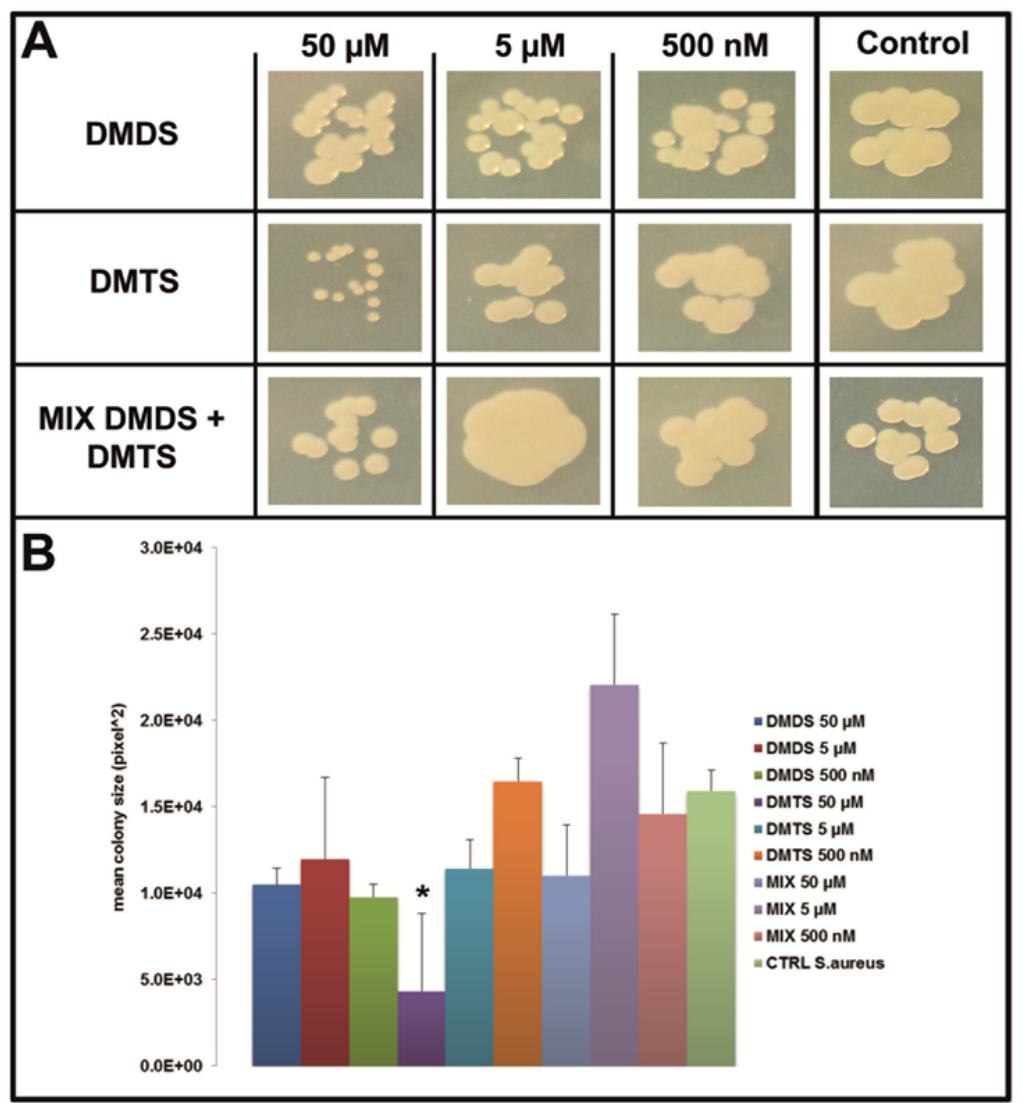

Figure 3.7: Effect of dimethyl disulfide (DMDS), dimethyl trisulfide (DMTS) and the mixture of both volatile compounds (DMDS + DMTS) (A) colony morphology and growth of $S$. aureus $533 \mathrm{R}_{4}$ after four days of incubation. The pure volatile compounds were applied in a concentration ranging from $500 \mathrm{nM}$ to $50 \mu \mathrm{M}$. Control $S$. aureus $533 \mathrm{R}_{4}$ grown without exposure to the compounds. (B) Mean colony sizes of $S$. aureus $533 R_{4}$ exposed to volatile compounds of dimethyl disulfide (DMDS), dimethyl trisulfide (DMTS) and the mixture of both volatile compounds (DMDS + DMTS). Asterisk indicates significant differences between the treatments and the control (ONE-WAY ANOVA, post-hoc Tukey test $\mathrm{p}<0.05$ ). Data represented are the mean of three replicates, error bars represent standard deviation of the mean. 


\section{Discussion}

Bacteria coexist with many different species in a heterogeneous and challenging soil environment (Gans et al., 2005). In this environment interspecific interactions between microorganisms are ongoing and are a key factor for their spatial distribution (Keller and Surette, 2006). To cope with the competitive conditions, bacteria developed different survival strategies such as the production of secondary metabolites with inhibitory capacity (Hibbing et al., 2010;Cornforth and Foster, 2013). Most of the studies on bacterial secondary metabolites so far were focused on non-volatile compounds (Korpi et al., 1998;Foster and Bell, 2012). However, bacteria do also release complex blends of volatile organic compounds. Yet, the effect of interspecific interactions on volatiles production and composition is still unknown (Garbeva et al., 2014a).

Here we compared the volatile blends emitted by four phylogenetically different soil-bacteria either grown in monocultures or in pairwise combinations. Our results revealed that the blend of volatiles emitted during pairwise combinations differed from the volatile blends of the respective monocultures. Yet, the volatile blend of the mixtures mostly included volatiles compounds produced by monocultures, although some compounds produced by the monocultures were not detected in mixtures. For example dimethyl sulfoxide produced by Tsukamurella sp. AD1o6 was not detected in the mixture with Chryseobacterium sp. $\mathrm{AD}_{4} 8$. Another interesting example is indole which was produced by the monocultures of Chryseobacterium sp. $\mathrm{AD}_{4} 8$ but was not detected in the presence of Tsukamurella sp. ADio6. Indole is a very well-studied compound and has been reported to be produced by about 85 different bacterial species including Chryseobacterium sp. (Yamaguchi and Yokoe, 200o;Lee and Lee, 2010). Indole and its derivatives (quinolones and (S)-3-hydroxytridecan-4-one) are involved in intercellular and multispecies signaling controlling diverse bacterial physiological properties like sporulation, plasmid stability, biofilm formation, drug resistance and virulence (Wang et al., 2001;Di Martino et al., 2003;Diggle et al., 2006;Nikaido et al., 2008;Lee et al., 2009;Lee and Lee, 2010). In addition, indole has been shown to have inhibitory activities on fungal growth (Aspergillus niger) and plant growth stimulating properties (A. thaliana) (Kamath and Vaidyanathan, 1990;Blom et al., 2011). In general indole is known to be a stable compound in the producing bacteria, however many non-indole producing bacteria are able to modify and to degrade indole (Shimada et al., 
2013;Lee et al., 2015). The fact that indole was not detected during the interaction of Chryseobacterium sp. AD48 with Tsukamurella sp. ADio6 suggests that the production of such signaling compounds in nature depends strongly on the interspecific interactions. Similar result was observed for the compound cyclopentene produced by the monocultures of Dyella sp. AD56 and Janthinobacterium sp. AD8o but not produced during the interaction of these two bacteria. With the volatolomic methods applied in this study we were able to detect 35 compounds from which 19 were tentatively identified. This discrepancy between numbers of detected and identified compounds shows that the identification of bacterial volatiles is yet a challenging and time demanding task, even with the use of sophisticated programs and software for metabolomics data analysis. Hence, the produced volatile blends are very complex and consist of a mixture of many unknown and difficult to identify compounds (Tait et al., 2014). Most of the volatile organic compounds that were tentatively identified within this study $(\sim 58 \%)$ contained sulfur (e.g. methyl thiocyanate, dimethyl disulfide, dimethyl trisulfide, dimethyl tetrasulfide etc.). The high abundance of sulfur containing volatiles in this study can be related to the cultivation of the tested bacteria on $1 / 10^{\text {th }}$ TSBA growth media. Several studies indicated that the composition of the volatile blend greatly depends on the growth media composition and the growth conditions (Schulz et al., 2004;Schulz and Dickschat, 2007;Blom et al., 2011;Garbeva et al., 2014b). The high amount of dimethyl di- and trisulfide detected in both monocultures and interactions indicate that these compounds are commonly produced. Many studies have shown that bacterial volatiles play a major role in soil fungistasis (Zou et al., 2007;Garbeva et al., 2011a;Garbeva et al., 2014b;van Agtmaal et al., 2015).

Indeed our results revealed that the fungal and oomycete tested organism are sensitive to bacterial volatiles and were inhibited significantly by all monocultures and pairwise combinations. The observed fungal and oomycetal growth inhibition is most probably related to sulfur containing volatiles. Sulfur containing volatiles like dimethyl di- and trisulfide have been shown to effect fungi and are able to inhibit the growth of different plant pathogenic fungi (Kai et al., 2009; Li et al., 2010;Huang et al., 2012;Wang et al., 2013;Garbeva et al., 2014b;Kanchiswamy et al., 2015).

While many study tested the effect of bacterial volatiles on various fungi, little is known so far on the effect of bacterial volatiles on other bacteria. In this study the volatiles emitted by Chryseobacterium sp. $\mathrm{AD}_{4} 8$ and the mixture of 
Chryseobacterium sp. $\mathrm{AD}_{4} 8$ with Tsukamurella sp. AD1o6 inhibited E. coli WA321. The observed growth promotion of $S$. aureus $533 \mathrm{R}_{4}$ was caused by the volatiles emitted by Dyella sp. AD56. However, this growth promotion was not observed by the volatiles emitted during the interaction of Dyella sp. AD56 with Janthinobacterium sp. AD8o correlating with a shift in volatile blend composition. Interestingly volatiles emitted by the monocultures of Chryseobacterium sp. $\mathrm{AD}_{4} 8$ and the mixture of Dyella sp. AD56 with Janthinobacterium sp. AD8o induced changes in colony morphology of S. marcescens P87. Our previous high-throughput screening for production of non-volatile antimicrobial compounds revealed that all four bacteria used here, showed induced antibacterial activity during pairwise interactions as compared to monocultures (Tyc et al., 2014). This was not observed in the present study, as we didn't observed novel produced volatile compounds during the pairwise interactions. Therefore it's questionable if volatiles solely play an important role as a competitive strategy between bacteria. However, it is possible that volatiles have synergistic or additive effect to other nonvolatile antibacterial compounds (Schmidt et al., 2015). Many bacteria are known to emit inorganic volatiles like $\mathrm{CO}_{2}, \mathrm{NH}_{3}, \mathrm{HCN}$, which also have biological activities and can have an additive effect (Effmert et al., 2012). However such compounds were not detected in this study as significant volatile compounds.

Here we tested two commonly produced bacterial volatile compounds for their effect on the target bacteria. The experiments with pure dimethyl trisulfide revealed strong growth inhibition on all tested bacterial model organisms, when applied in a concentration of $50 \mu \mathrm{M}$. Bacterial growth suppression was already reported for dimethyl disulfide emitted by Pseudomonas strains against the crown-gall diseases causing Agrobacterium sp. (Dandurishvili et al., 2011;Popova et al., 2014). Dimethyl trisulfide effected colony morphology and pigmentation in $S$. marcescens $\mathrm{P} 87$ when applied in a concentration of $50 \mu \mathrm{M}$. Volatiles exposed colonies showed reduced growth and white coloration indicating the lack of prodigiosin production. It is plausible that this observation is related to the inhibition of quorum sensing as previously reported by Moroshi and Chernin (Morohoshi et al., 2007; Chernin et al., 2011).

However, the effective concentration of $50 \mu \mathrm{M}$ dimethyl trisulfide is most probably very high and far away from the concentrations in which those volatile compounds are produced in nature (Groenhagen et al., 2013) as we did 86 
not observed this effect in the experiments where $S$. marcescens P87 was exposed to the volatile blend produced by bacteria. The biological relevant concentration of volatile compounds remains to be determined in future studies.

In conclusion, this work revealed that interspecific bacterial interactions affect volatile blend composition. This observed change is most probably related to the combination of volatile compounds produced by each isolate rather than triggering the production of novel volatiles as the volatile blend was composed of the mixture of the respective interacting bacteria. Furthermore the loss of production of certain compounds during pairwise interaction suggests that the production of volatile signaling compounds (e.g. indole) in nature is influenced by interspecific interactions. While fungi and oomycetes showed to be very sensitive to bacterial volatiles the effect of volatiles on bacteria varied greatly between no effects, growth inhibition to growth promotion depending on the volatile blend composition.

\section{Acknowledgment}

This work is supported by the BE-Basic Foundation (http://www.be-basic.org/). P.G. is financed by The Netherlands Organization for Scientific Research (NWO) VIDI personal grant (864.11.015). The authors want to thank Saskia Gerards for her great help during experimentation and Dr. Kees Hordijk for his help with GC/MS data analysis. This is publication 5957 of the NIOO-KNAW. 


\section{Supplementary material}

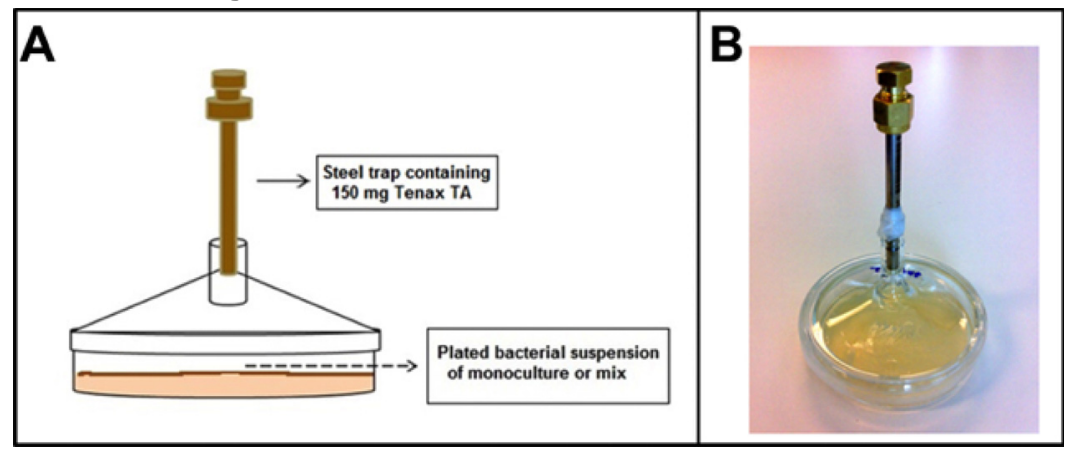

Figure S3.1: Used glass-petri dish system for bacterial volatile organic compounds (VOCs) trapping. (A) Illustration of the glass-petri dish; (B) photograph of a inoculated glass-petri dish with a connected Tenax TA steel trap for volatile organic compound trapping.

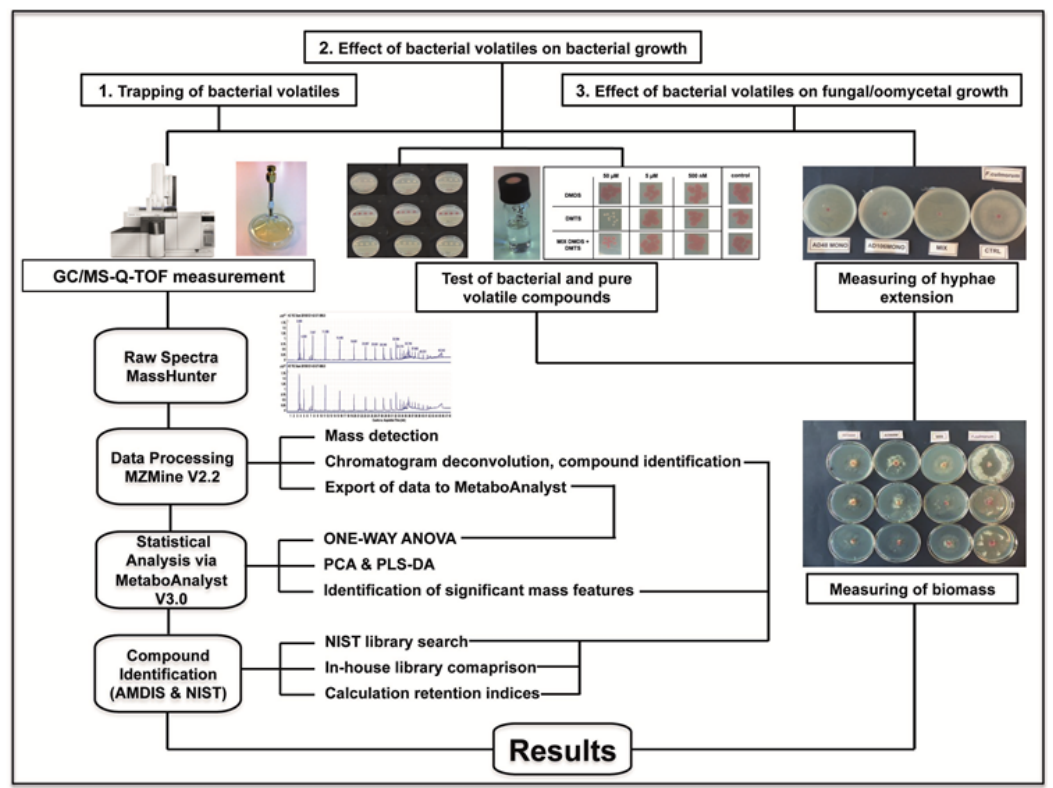

Figure S3.2: Workflow of the volatolomics analysis performed in this study. In total three experimental parts were carried out: 1. Volatile trapping and GC/MS-Q- TOF combined with statistical analysis, 2. Effect of bacterial volatiles on bacterial growth and colony morphology and 3. Effect of bacterial volatiles on fungal and oomycetal model organisms. 


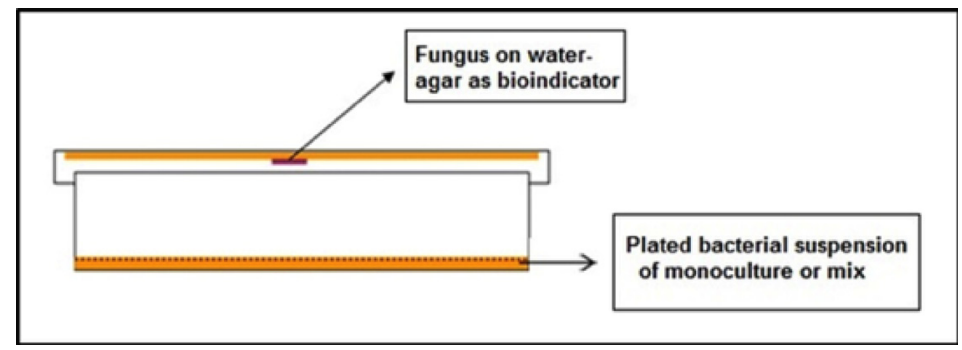

Figure S3.3: Used top-bottom-petri dish system for experiments to elucidate fungal inhibitory capacities of the produced bacterial volatiles.
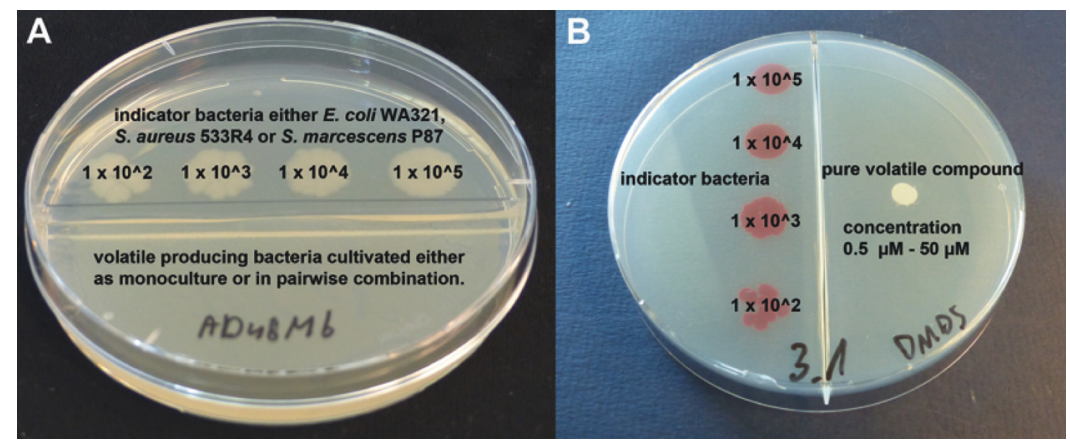

Figure S3.4: Two-compartment petri dish system used in bacterial volatile and pure volatile compound experiments. (A) to elucidate the effect of bacterial volatiles on growth and colony morphology of E. coli WA321, S. aureus $533 \mathrm{R}_{4}$ and $S$. marcescens $\mathrm{P} 87$ (B) to elucidate the effect of pure volatile compounds dimethyl di- and tri- sulfide on growth and colony morphology on E. coli WA321, S. aureus 533R4 and S. marcescens P87. 


\section{Volatiles in interspecific bacterial interactions}

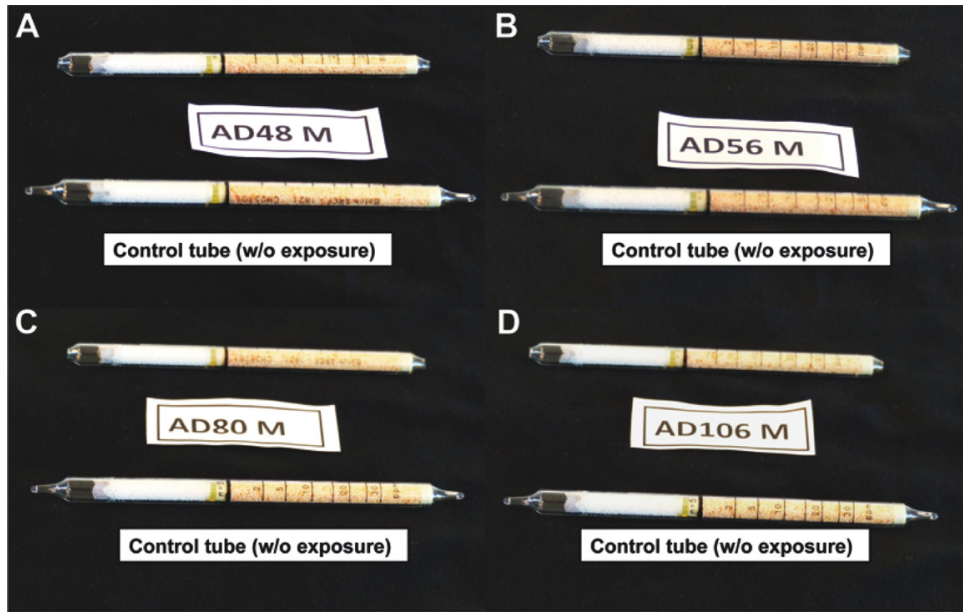

Figure S3.5: Hydrocyanic-acid tests using Dräger Hydrocyanic acid test tubes in combination with the Dräger accuro $^{\circledR}$ gas detection pump. All bacterial strains were tested negative for the production of Hydrocyanic acid (<2 ppm). Test result for: (A) Chryseobacterium sp. AD48. (B) Dyella sp. AD56 (C) Janthinobacterium sp. AD8o. (D) Tsukamurella sp. AD1o6.

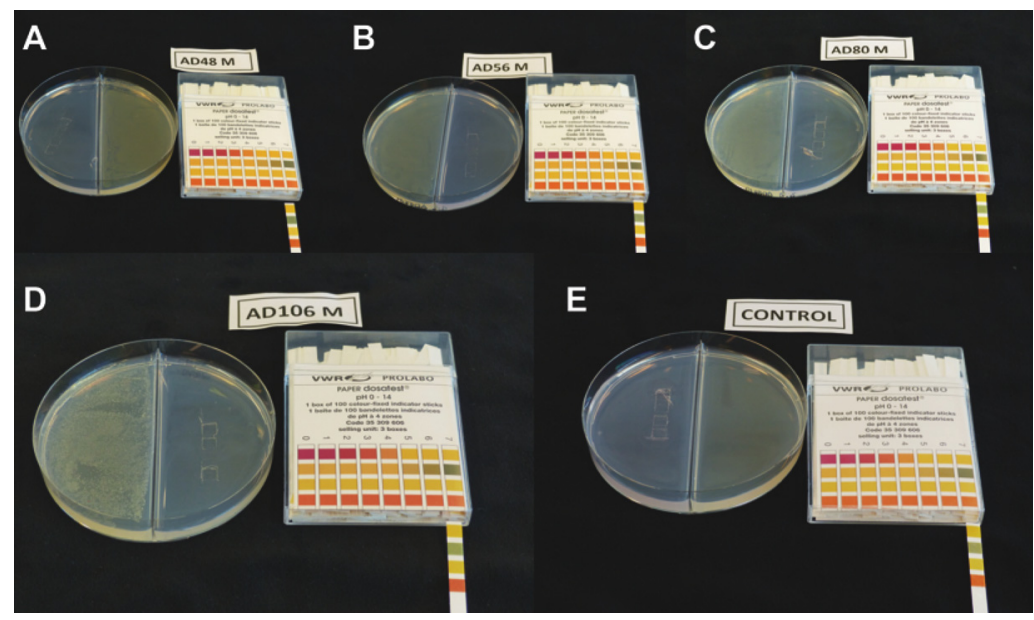

Figure S3.6: $\mathrm{pH}$ - tests using VWR PROLABO dosatest ${ }^{\circledR} \mathrm{pH}$ - test strips. All bacterial strains did not change the $\mathrm{pH}$ - value of the growth medium where the target organisms were grown $(\mathrm{pH}=7)$. Test result for: (A) Chryseobacterium sp. AD48. (B) Dyella sp. AD56 (C) Janthinobacterium sp. AD8o. (D) Tsukamurella sp. AD1o6. (E) The control, two compartment petri dish without exposure to bacterial volatiles. 


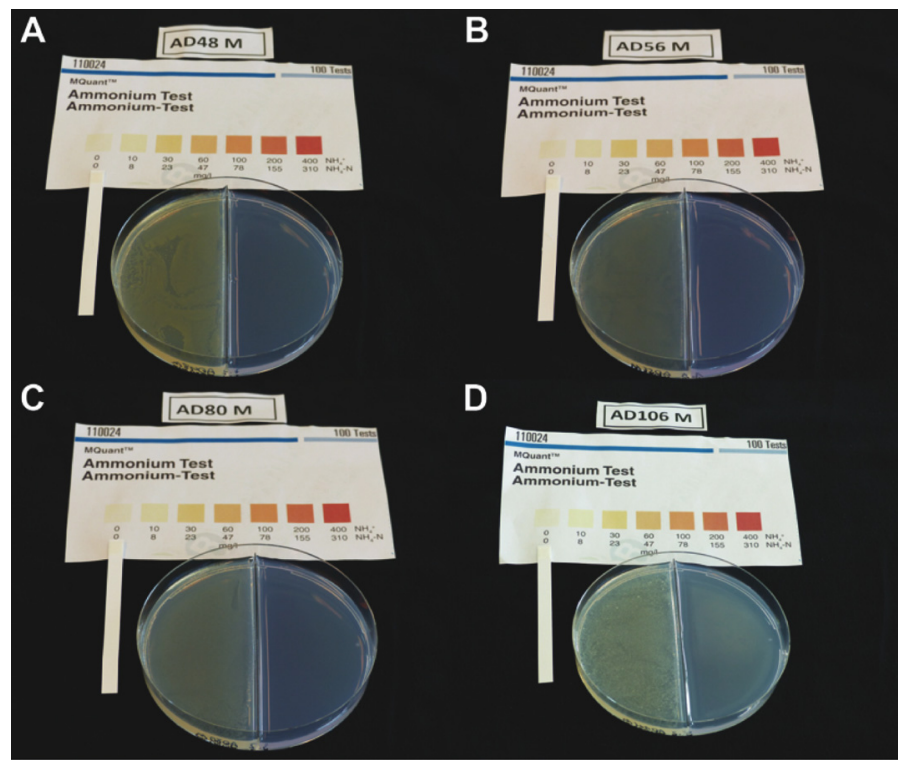

Figure S3.7: Measurements of ammonia emission using the MQuant ${ }^{\mathrm{TM}}$ ammonium test kit. All bacterial strains were tested negative for the production of ammonium. Test result for: (A) Chryseobacterium sp. AD48. (B) Dyella sp. AD56 (C) Janthinobacterium sp. AD8o. (D) Tsukamurella sp. ADio6. 


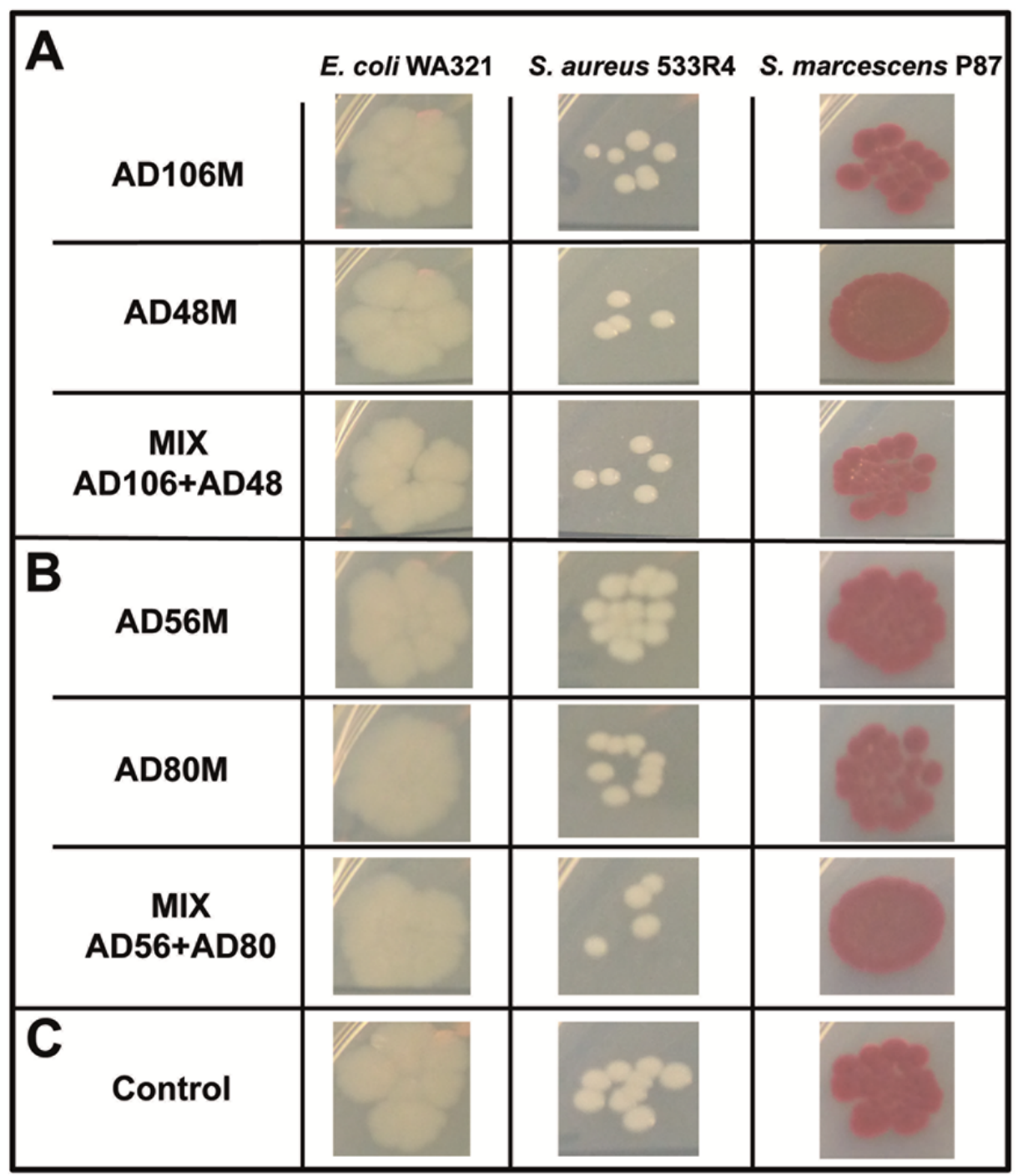

Figure S3.8: Effect of bacterial volatile compounds on colony morphology on the three model organisms: E.coli WA321, S.aureus $533 \mathrm{R}_{4}$ and S. marcescens P87. (A) Effect of the produced volatile blend of Tsukamurella sp. ADı6, Chryseobacterium sp. AD48 and the interaction of both bacteria (B) Effect of the produced volatile blend of Dyella sp. AD56, Janthinobacterium sp. AD8o and the interaction of both bacteria. (C) Control without exposure to bacterial volatiles. 


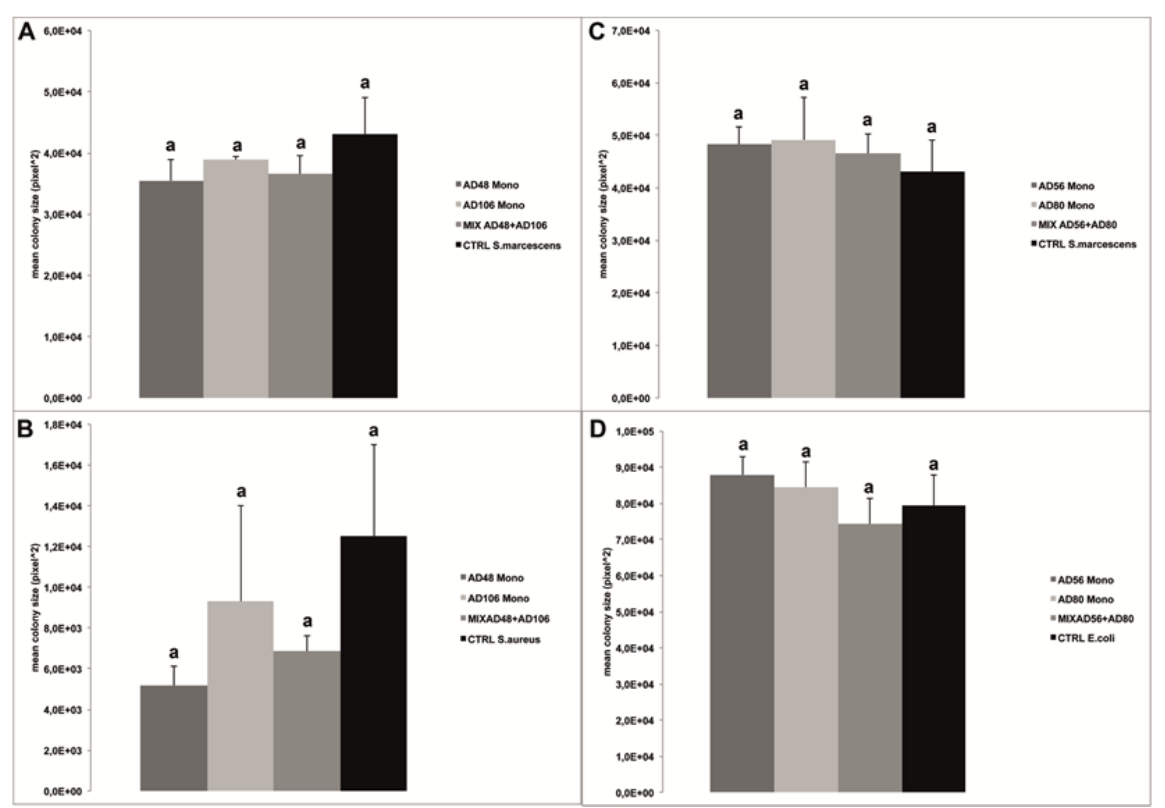

Figure S3.9: Average colony size of the target organisms. (A) Mean colony size of $S$. marcescens P87 exposed to volatile compounds of Chryseobacterium sp. AD48 and Tsukamurella sp. AD1o6 and the interaction of both bacteria. (B) Mean colony size of $S$. aureus $533 \mathrm{R}_{4}$ exposed to volatile compounds of Chryseobacterium sp. AD48 and Tsukamurella sp. AD1o6 and the interaction of both bacteria. (C) Mean colony size of $S$. marcescens P87 exposed to volatile compounds of Dyella sp. AD56, Janthinobacterium sp. AD8o and the interaction of both bacteria. (D) Mean colony size of E. coli WA321 exposed to volatile compounds of Dyella sp. AD56, Janthinobacterium sp. AD8o and the interaction of both bacteria. Data represented are the mean of three replicates, error bars represent standard deviation (SD). 
Volatiles in interspecific bacterial interactions 
Chapter

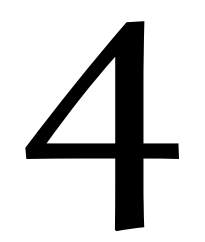

\section{Exploring bacterial interspecific interactions for discovery of novel antimicrobial compounds}

Olaf Tyc, Victor de Jager, Marlies van den Berg, Saskia Gerards, Thierry Janssens, Niels Zaagman, Marco Kai, Ales Svatos, Hans Zweers, Cornelis Hordijk, Harrie Besselink, Wietse de Boer and Paolina Garbeva

Submitted for Publication 


\title{
Exploring bacterial interspecific interactions for discovery of novel antimicrobial compounds
}

\begin{abstract}
In terrestrial ecosystems bacteria live in close proximity with many species and form complex communities. Recent studies indicated that the production of secondary metabolites by soil bacteria can be triggered by interspecific interactions. However, little is known about the mechanisms involved in interspecific interactions, in particular for interactions between Gram-positive and Gram- negative bacteria.

In this study we aimed to understand how the interspecific interaction between Paenibacillus sp. AD87 and Burkholderia sp. AD24 affects fitness, gene expression and metabolism. To facilitate the transcriptomic and metabolomics analysis both bacteria were subjected to genome and RNAsequencing.

Our results revealed that the interaction between the two bacteria affected their gene expression, fitness and the production of secondary metabolites. The growth of Burkholderia was more affected during the interspecific interaction than that of Paenibacillus. One volatile and a soluble compound were produced in higher amounts during interaction but not in the monocultures. The identified volatile compound was subjected to bioassays and showed strong inhibitory activity against a range of plant and human pathogens.
\end{abstract}

Our results highlight the importance of interspecific interactions for triggering secondary metabolites production for the discovery of novel useful bioactive compounds important for agriculture as well for medical purposes. 


\section{Introduction}

Recent studies have shown that interspecific interactions between soil bacteria strongly affect their behavior such as the induced secretion of secondary metabolites (Seyedsayamdost et al., 2012;Traxler et al., 2013;Tyc et al., 2014). The soil and rhizosphere are environments characterized by high complexity, diversity and density of microorganisms (Gans et al., 2005; Uroz et al., 2010). In these environments microorganisms can interact in different ways. These interactions are lively and range from competition to cooperation (Czaran and Hoekstra, 2009;Foster and Bell, 2012;Allen and Nowak, 2013). Many soil bacterial species have overlapping metabolic niches i.e. they use similar substrates as an energy source for their growth and persistence (Yin, 2000;Demoling et al., 2007;Strickland, 2009). Consequently, competition for nutrients is one of the most abundant forms of interaction occurring in soil and rhizosphere bacterial communities (Demoling et al., 2007;Rousk et al., 2009). To sustain in such demanding environmental conditions bacteria evolved the ability to produce a wide range of secondary metabolites with antimicrobial properties (e.g. antibiotics, siderophores, bacteriocins, volatiles and others) as competitive tools (Hibbing et al., 2010). Bacteria with the ability to produce antimicrobial compounds can also contribute to crop health by suppression of plant-pathogenic micro-organisms (Raaijmakers and Mazzola, 2012;Mendes et al., 2013). Hence, comprehensive knowledge of bacterial interspecific interactions is important for better understanding of soil microbial community composition and soil functions such as disease suppression and plant growth promotion.

Previously we have performed a high-throughput screening of interactionmediated production of antimicrobial compounds by rhizobacterial strains (Tyc et al., 2014). A clear case of such interaction-mediated triggering of antibiotic production was observed when a Burkholderia and a Paenibacillus strain were co-cultured. So far, very little is known on interactions between Gram-positive and Gram-negative bacteria and the triggering of secondary metabolite production during such interactions. Bacteria belonging to the genus Burkholderia are Gram-negative, non-spore forming and belong to the phylum Proteobacteria. These bacteria are able to occupy a diverse range of ecological niches (Salles et al., 2002;van Elsas et al., 2002;Coenye and Vandamme, 2003;Compant et al., 2008). The lifestyle of Burkholderia spp. can range from free living in soil and rhizosphere to endo- and epiphytic, including obligate endosymbionts and plant pathogens (Coenye and Vandamme, 2003;Compant et al., 2005;Vial et al., 2011). In recent years the 


\section{Exploring bacterial interspecific interactions for discovery of novel antimicrobial compounds}

interest on Burkholderia strains has increased as these bacteria have shown to have compelling properties for agriculture like plant growth promotion, increasing of diseases resistance, improvement of nitrogen fixation and phosphorus utilization (Nowak and Shulaev, 2003;Sessitsch et al., 2005;Schmidt et al., 2009;Groenhagen et al., 2013;Zhao et al., 2014).

Soil bacteria belonging to the genus Paenibacillus are Gram positive, facultative anaerobe and endo-spore forming bacteria (von der Weid et al., 2000;da Mota et al., 2005). Many paenibacilli are known to act as PGPR in agricultural systems (Anand et al., 2013). Bacteria of this genus are able to colonize diverse habitats like water, soil and insects (Berge et al., 2002;Bosshard et al., 2002;Daane et al., 2002;Peters et al., 2006;Timmusk et al., 2009). Many studies have shown that paenibacilli play an important role in soil for plant health and growth (e.g. nitrogen fixing, pest control) (McSpadden Gardener, 2004;Ryu et al., 2005;Debois et al., 2013). Furthermore members of the genus Paenibacillus are known as a rich source for chemical compounds useful in the field of biotechnology and agriculture such as antibiotics, enzymes and other bioactive molecules (Wu et al., 2010;Debois et al., 2013; Cochrane and Vederas, 2016).

The main goal of our study was to obtain insight in the mechanisms and consequences of interspecific interaction between Burkholderia sp. AD24 and Paenibacillus sp. AD87. The major research questions were how this interspecific interaction will affect bacterial cell numbers and fitness, gene expression, the production of soluble and volatile secondary metabolites and their activity. 


\section{Materials and methods}

\section{Bacteria and culture conditions}

Based on a previous screening (Tyc et al., 2014) a Gram-negative strain, Burkholderia sp. AD24 (Beta-proteobacteria), and a Gram-positive strain, Paenibacillus sp. AD87 (Firmicutes), were selected for this study. The bacterial isolates were pre-cultured from $-80{ }^{\circ} \mathrm{C}$ glycerol stocks on $1 / 10^{\text {th }}$ TSBA (Garbeva and de Boer, 2009) and incubated for three days at $24{ }^{\circ} \mathrm{C}$.

Two indicator bacteria, E. coli WA321 and S. aureus $533 \mathrm{R}_{4}$, were used as target bacteria to detect the production of compounds with antibacterial activity (Meyer and Schleifer, 1978; Tyc et al., 2014). The bacteria were pre-cultured from $-80{ }^{\circ} \mathrm{C}$ glycerol stocks on LB-A media (LB-Medium Lennox, Carl Roth $\mathrm{GmbH}+\mathrm{Co}$. KG, The Netherlands, solidified with $20 \mathrm{gL}^{-1}$ Merck Agar). The indicator bacteria were incubated overnight at $37{ }^{\circ} \mathrm{C}$ prior application. All bacterial isolates used in this study are listed in Table 4.1.

\section{Eukaryotic model organisms and culture conditions}

The plant pathogens Rhizoctonia solani AG2.2IIIB and Fusarium culmorum PV were used as fungal model organisms (Garbeva et al., 2014b). The fungi were pre-cultured on $1 / 5^{\text {th }}$ Potato Dextrose Agar (PDA) (29 $\mathrm{gL}^{-1}$ Oxoid CM 139) (Fiddaman and Rossall, 1993) and incubated at $24{ }^{\circ} \mathrm{C}$ for 7 days. As a model organism for yeast-like fungi Candida albicans BSMY 212 (DSMZ \# 10697) was used. C. albicans BSMY 212 (Schmidt, 1996) was pre-cultured from $-80{ }^{\circ} \mathrm{C}$ glycerol stocks on YEPD plates $\left(20 \mathrm{gL}^{-1}\right.$ Merck Dextrose, $20.0 \mathrm{gL}^{-1} \mathrm{BACTO}^{\mathrm{TM}}$ Peptone, $10.0 \mathrm{gL}^{-1}$ BACTO ${ }^{\mathrm{TM}}$ Yeast extract, $20 \mathrm{gL}^{-1}$ Merck Agar). All eukaryotic model organisms are listed in Table 4.1.

\section{Experimental treatments}

For all experiments three different treatments were performed in triplicates at three time points. These treatments were: monoculture AD24M (Burkholderia), monoculture AD87M (Paenibacillus) and the interaction of Burkholderia + Paenibacillus. 


\section{Exploring bacterial interspecific interactions for discovery of novel antimicrobial compounds}

Table4. 1: Bacterial and fungal strains used in this study.

\begin{tabular}{|c|c|c|c|c|}
\hline Strain / isolate / organism & Phylum/class & Genbank & Reference & Function \\
\hline \multicolumn{4}{|l|}{ Bacteria tested during this study } & \multirow{3}{*}{$\begin{array}{c}\text { used in interaction } \\
\text { assays, RNA isolation } \\
\text { and in LC/MS \& GC/MS } \\
\text { measurements }\end{array}$} \\
\hline Burkholderia sp. AD24 & beta-proteobacteria & KJ685239 & Tyc et al. 2014 & \\
\hline Paenibacillus sp. AD87 & Firmicutes & KJ685299 & Tyc et al. 2014 & \\
\hline \multicolumn{5}{|l|}{ Eukaryotic model organisms } \\
\hline $\begin{array}{l}\text { Candida albicans BSMY } 212 \\
\text { DSMZ } 10697\end{array}$ & Saccharomycetes & & Schmidt 1996 & \multirow{3}{*}{$\begin{array}{l}\text { eukaryotic model } \\
\text { organisms for growth } \\
\text { inhibition }\end{array}$} \\
\hline Fusarium culmorum PV & Ascomycota & - & Garbeva et al. 2014 & \\
\hline Rhizoctonia solani AG2.2IIIB & Basidiomycota & KT124637 & Garbeva et al. 2011 & \\
\hline \multicolumn{5}{|l|}{ Bacterial model organisms } \\
\hline $\begin{array}{l}\text { Escherichia coli WA321 } \\
\text { DSMZ } 4509\end{array}$ & $\begin{array}{l}\text { gamma- } \\
\text { proteobacteria }\end{array}$ & - & Tyc et al. 2014 & \multirow{2}{*}{$\begin{array}{l}\text { bacterial model } \\
\text { organisms for growth } \\
\text { inhibition }\end{array}$} \\
\hline $\begin{array}{l}\text { Staphylococcus aureus 533R4 } \\
\text { Serovar } 3 \text { DSMZ } 20231\end{array}$ & Firmicutes & LN681573 & Meyer et al. 1978 & \\
\hline
\end{tabular}

\section{Bacterial interaction assay}

After three-days of pre-cultivation a single colony of each bacterial isolate was picked and inoculated in $20 \mathrm{~mL} 1 / 10^{\text {th }}$ TSB broth and grown overnight at $24{ }^{\circ} \mathrm{C}$, $220 \mathrm{rpm}$ to an optical density of $\mathrm{OD}_{6 \text { оо }} 0.630$ (Burkholderia) and $\mathrm{OD}_{6 \circ 0} 0.680$ (Paenibacillus). An inoculation mix for each treatment was prepared by diluting the bacterial isolates in $20 \mathrm{~mL} 10 \mathrm{mM}$ Phosphate-buffer ( $\mathrm{pH} \mathrm{6.5)}$ to an starting $\mathrm{OD}_{600}$ of 0.002 (Burkholderia) and 0.005 (Paenibacillus) corresponding to $\sim 5 \times 10^{5} \mathrm{CFU} / \mathrm{mL}$. Each inoculation mix was pulse- vortexed for $30 \mathrm{sec}$. and a volume of $100 \mu \mathrm{l}$ (monoculture or mixture) was spread on $1 / 10^{\text {th }}$ TSBA plates (20 mL of TSBA). Plates were incubated four three days at $24^{\circ} \mathrm{C}$ and sampling for bacterial cell counts, total RNA and secondary metabolite extraction was performed after 24 hours, 48 hours and 72 hours of incubation.

\section{Enumeration of bacteria growth}

The growth of the two bacterial strains was tracked by selective plate counting. After 24 hours, 48 hours and 72 hours of incubation a volume of 1 $\mathrm{mL}$ of $10 \mathrm{mM}$ phosphate buffer ( $\mathrm{pH} 6.5$ ) was added to the surface of the $1 / 10^{\text {th }}$ TSBA plates and cells were suspended from the plate surface with a disposable cell scratcher (VWR international B.V., Amsterdam, The Netherlands Cat\# 50806-404). The cell suspension was transferred to a $15 \mathrm{~mL}$ Greiner tube containing $9 \mathrm{~mL}$ of $10 \mathrm{mM}$ phosphate buffer ( $\mathrm{pH}$ 6.5) and homogenized by pulse vortex for $30 \mathrm{sec}$. Dilution series of each treatment were prepared in triplicates. A volume of $100 \mu \mathrm{l}$ of each serial dilution were plated in three replicates with a disposable drigalski spatula on $1 / 10^{\text {th }}$ TSBA plates supplemented with either Streptomycin $25 \mu \mathrm{g} / \mathrm{mL}$ (plates for selection of 100 
Paenibacillus) or Vancomycin $50 \mu \mathrm{g} / \mathrm{mL}$ (plates for selection of Burkholderia). The plates were incubated for three days at $20{ }^{\circ} \mathrm{C}$. Bacterial enumeration was carried out on an aCOlyte Colony Counter (Don Whitley Scientific, Meintrup DWS Laborgeräte GmbH, Germany).

\section{RNA sampling and isolation}

For RNA- extraction $0.5 \mathrm{~mL}$ of each sample was transferred to an $2 \mathrm{~mL}$ tube containing $1 \mathrm{~mL}$ RNA protect Bacteria Reagent (Qiagen B.V., Venlo, The Netherlands, cat\# 76506) and centrifuged for $10 \mathrm{~min}$. at 10,0oo x g, and $4{ }^{\circ} \mathrm{C}$ (Sigma $3 \mathrm{~K}-14$ centrifuge, SIGMA Laborzentrifugen GmbH, Germany). The supernatant was discarded and cells were directly frozen in liquid $\mathrm{N}_{2}$ and immediately stored at $-8 \mathrm{o}^{\circ} \mathrm{C}$. Total RNA was extracted with the Aurum Total RNA Mini Kit (BIO- RAD cat\# 732-6820) according to the manufacturer's protocol. Samples were treated with the TURBO DNA free Kit (AMBION cat\# 1907) according to the manufacturer's protocol. The RNA concentration and quality was checked on a NanoDrop Spectrophotometer (Isogen Life Science, IJssestein, The Netherlands) and on a $1.0 \%$ agarose gel. Samples were subjected to RNA- sequencing at Baseclear (BASECLEAR, Leiden, The Netherlands) using the Illumina Sequencing platform.

\section{Whole genome DNA isolation and genome sequencing}

Genomic DNA of Burkholderia and Paenibacillus was extracted from overnight cultures by using the QIAGEN genomic DNA Mini Kit (Qiagen B.V., Venlo, The Netherlands, cat\# 13323) according to the manufacturer's protocol (for Illumina Sequencing) or by using the QIAGEN MagAttract HMW DNA Kit (Qiagen B.V., Venlo, The Netherlands, cat\# 67563) from exponentially growing overnight cultures for PacBio Sequencing. The extracted DNA was dissolved in $100 \mu \mathrm{l}$ sterile nuclease free water and quantified with a NanoDrop Spectrophotometer (Isogen Life Science, IJsselstein, The Netherlands). Additionally a $1.0 \%$ agarose gel in $0.5 \%$ TBE buffer was run to check the size and integrity of the isolated DNA. The extracted genomic DNA was stored at $-20{ }^{\circ} \mathrm{C}$ and subjected for genomic DNA sequencing at Baseclear (BASECLEAR, Leiden, The Netherlands) using the Illumina Sequencing platform and to the Institute for Genome Sciences (IGS), Baltimore, Maryland, USA for Pacbio real-time DNA sequencing.

\section{De Novo assembly of Paenibacillus sp. AD87 and Burkholderia sp. AD24 genomes}

From the paired-end Illumina sequencing platform an average read length of 101 bp was obtained and from the Pacbio RS platform (Pacific Biosciences, 


\section{Exploring bacterial interspecific interactions for discovery of novel antimicrobial compounds}

Menlo Park, CA, USA) using the $\mathrm{P}_{4}-\mathrm{C}_{2}$ chemistry an average read length of 8184 nucleotides for Paenibacillus sp. AD87 and 8334 for Burkholderia was obtained. The Pacbio raw sequences were analyzed using SMRT portal V2.3.0.140936. p.4150482. The sequences were assembled with RS_HGAP_assembly 3 protocol (ㄷ Copyright 2010 - 2014, Pacific Biosciences, Menlo Park, CA, USA) at default settings with estimated genome sizes of 7 MBp for Paenibacillus and $8 \mathrm{MBp}$ for Burkholderia. The resulting assemblies were subjected to scaffolding using the RS_AHA_scaffolding 1 protocol. The Illumina reads were filtered using Fastq MCF with default settings and aligned against the scaffolds using BWA Vo.7.12. The aligned reads were re-aligned with GATK V3.5.o. The scaffolds of both genomes were corrected using the realigned reads with Pilon (Walker et al. 2014). The resulting improved contigs were subjected to another round of scaffolding in SMRT-portal and further corrected. The whole genome assembly properties are shown in Table S4.1. The final contigs were annotated using a modified version of PROKKA V1.11 (Seemann, 2014) and InterproScan 5.16 55.o (Jones et al., 2014). Both genomes were submitted to the NCBI genome database under accession numbers NCBI PRJNA320371 (Burkholderia sp. AD24) and NCBI: LXQNoooooooo (Paenibacillus sp. AD87).

\section{Transcriptome and in-silico secondary metabolite analysis}

The obtained Illumina reads from the RNA- sequencing were filtered using Fastq MCF and aligned against the cDNA sequences of both Burkholderia and Paenibacillus combined using Bowtie 2 (Langmead and Salzberg, 2012) with the following settings: - no-mixed - no-discordant - gbar 1000 - end-to-end. Transcript abundance was estimated using RSEM V1.1.26 (Li and Dewey, 2011) and differential expression between the treatments was analyzed using edgeR V3.2 (Robinson et al., 2010;Zhou et al., 2014). Data were filtered with a p-value of o.oo1. For in silico analysis of secondary metabolite gene clusters the antiSMASH website (http://antismash.secondarymetabolites.org/) (Medema et al., 2011) was used.

\section{COG annotation}

COG annotations were determined for both Burkholderia and Paenibacillus by using custom scripts based on a modified version of methods described by Snel (Snel et al., 2002) with COG annotations from Galperin (Galperin et al., 2015). 


\section{Reverse transcription and quantitative real time PCR}

To confirm the RNA- Sequencing results the gene expression of two gene clusters related to secondary metabolite production (a Pederin like compound in Paenibacillus and a polyketide synthase in Burkholderia) were targeted and quantified via quantitative real time PCR. For this purpose the previously extracted RNA was used to synthesize first strand cDNA by using the SuperScript ${ }^{\circledast}$ VILO $^{\mathrm{TM}}$ MasterMix (Invitrogen, cat\#11755050). The concentration and quality of the cDNA was determined using a NanoDrop ${ }^{\text {TM }}$ spectrophotometer (Isogen Life Science, IJssestein, the Netherlands), additionally all cDNA samples were run on a $1.0 \%$ agarose gel to check the size and integrity.

The selected gene cluster in Burkholderia was targeted with the following primer combinations bAD24_10391_IG_F (5'GTATTGGCCGTATCCGTCAG) and bAD24_10391_IG_R (5'AGCCACTCTTCGACGATCAC) amplifying 322 bp from gene bAD24_10391 encoding snoaL-like polyketide cyclase family protein. For normalization the two primers Eub338F (5'ACTCCTACGGGAGGCAGCAG) with Eub518R (5'ATTACCGCGGCTGCTGG) amplifying $180 \mathrm{bp}$ from the $16 \mathrm{~S}$ rRNA gene (Fierer et al., 2005) and primers RecA_bAD24_1_F (5' GGTGAGGCAATCGAAGACAT) with RecA_bAD24_1_R (5'AGCTTGCTTGCGTACTGGAT) amplifying $230 \mathrm{bp}$ from gene recA encoding DNA recombination and repair protein were used.

The gene clusters in Paenibacillus were targeted with the primer combination gpAD87_304F (5' GTACTTCCCGCACCTGACAT) and gpAD87_304R (5'TGGCGAGAAACTCCACTTCT) amplifying 592 bp from gene encoding a dimodular nonribosomal peptide synthase. For normalization the two primers BacF (5'GGGAAACCGGGGCTAATACCGGAT) (Garbeva et al., 2003) with Eub518R (5'ATTACCGCGGCTGCTGG) amplifying about 440 bp from the $16 \mathrm{~S}$ rRNA gene and primers RecA_gpAD87_3_F (5'CTTGCCTAAAGGCCGTATTG) and RecA_gpAD87_3_R (5'GACAATGTCCACAGCACCAC) amplifying 259 bp from gene recA encoding DNA recombination and repair protein were used. From each treatment $5 \mu$ l cDNA were subjected to quantitative RT- PCR using QuantiNova SYBR Green I PCR master mix (Qiagen B.V., Venlo, The Netherlands, Cat\# 208056) or with iTaq $^{\mathrm{TM}}$ Universal SYBR $^{\circ}$ green (BIORAD Cat\# 1725122). For quantification two-step quantitative RT- PCRs were performed on a Qiagen Research Rotor- Gene Q thermal cycler (Qiagen B.V., Venlo, The Netherlands, Cat\# 9001550), with the following settings: initial cycle $95^{\circ} \mathrm{C}$ for $2 \mathrm{~min}$., followed by 40 cycles of $95^{\circ} \mathrm{C}$ for $5 \mathrm{sec}$. (denaturation) and $60^{\circ} \mathrm{C}$ for 10 sec. (combined annealing/extension). All analyses were performed in triplicate. Five standard curves were established to calculate the 


\section{Exploring bacterial interspecific interactions for discovery of novel antimicrobial compounds}

expression (CT-value). Gene expression was calculated relative to the $16 \mathrm{~S}$ rDNA and recA gene of both, Burkholderia and Paenibacillus by using the 2- $\Delta \Delta \mathrm{Ct}$ method (Livak and Schmittgen, 2001). All used primers for qPCR analysis are listed in Table $\mathbf{S}_{\mathbf{4} .2}$.

\section{Trapping of volatile organic compounds}

For analysis of volatile organic compounds glass Petri dishes designed for trapping headspace volatiles (Garbeva et al., 2014b) were used. The Petri dishes were closed by a lid with an outlet connected to a steel trap containing 150 mg Tenax TA and 150 mg Carbopack B (Markes International Ltd., Llantrisant, UK). The Tenax steel traps containing the volatile compounds were collected after 72 hours of incubation and stored at $4{ }^{\circ} \mathrm{C}$ until GC-Q-TOF analysis. As controls glass Petri dishes containing $1 / 10^{\text {th }}$ TSBA media without inoculated bacteria were used as background control in GC/MS measurements.

\section{GC-Q-TOF analysis}

The trapped volatile organic compounds were desorbed from the traps using an automated thermos desorption unit (Unity TD-10o, Markes International Ltd., Llantrisant, UK) at $210^{\circ} \mathrm{C}$ for $12 \mathrm{~min}$ (He flow $50 \mathrm{~mL} / \mathrm{min}$ ) and trapped on a cold trap at $-10{ }^{\circ} \mathrm{C}$. The trapped volatiles were introduced into the GCQTOF (model Agilent 789oB GC and the Agilent 7200A QTOF, Santa Clara, USA) by heating the cold trap for $12 \mathrm{~min}$ to $250^{\circ} \mathrm{C}$. Split ratio was set to 1:10, and the column used was a $30 \times 0.25 \mathrm{~mm}$ ID RXI-5MS, film thickness $0.25 \mu \mathrm{m}$ (Restek 13424-6850, Bellefonte, PA, USA). Temperature program used was as follows: $39^{\circ} \mathrm{C}$ for $2 \mathrm{~min}$, from $39{ }^{\circ} \mathrm{C}$ to $95{ }^{\circ} \mathrm{C}$ at $3.5^{\circ} \mathrm{C} / \mathrm{min}$, then to $165^{\circ} \mathrm{C}$ at $4{ }^{\circ} \mathrm{C} / \mathrm{min}$, to $280{ }^{\circ} \mathrm{C}$ at $15{ }^{\circ} \mathrm{C} / \mathrm{min}$ and finally to $320^{\circ} \mathrm{C}$ at $30^{\circ} \mathrm{C} / \mathrm{min}$, hold $7 \mathrm{~min}$. A constant gas flow of $1.2 \mathrm{ml} / \mathrm{min}$ was used. Volatile organic compounds were ionized in EI mode at eV. Mass spectra were acquired in full-scan-mode (30400 U @ 5 scans/s). Mass-spectra were extracted with MassHunter Qualitative Analysis Software V B.o6.oo Build 6.o.633.o (Agilent Technologies, Santa Clara, USA). The obtained mass spectra's were exported as mzXML files for further processing in MZmine V2.14.2. The files were imported to MZmine V2.14.2 (Copyright @ 2005-2012 MZmine Development Team) (Pluskal et al., 2010) and compounds were identified via their mass spectra using deconvolution function (Local-Maximum algorithm) in combination with two mass-spectral-libraries: NIST 2014 V2.20 (National Institute of Standards and Technology, USA http://www.nist.gov) and Wiley 7 th edition spectral 
libraries and by their linear retention indexes (LRI). The LRI values were calculated using AMDIS 2.72 (National Institute of Standards and Technology, USA). After deconvolution and mass identification peak lists containing the mass features of each treatment (MZ-value/Retention time and peak intensity) was saved in csv file format and uploaded to Metaboanalyst V3.0 (www.metaboanalyst.ca).

\section{Extraction of secondary metabolites}

Secondary metabolites were extracted from the samples used for bacterial enumeration and RNA isolation. The agar was cut into pieces of about $2 \mathrm{~cm}^{2}$ and transferred to $50 \mathrm{~mL}$ Greiner tubes. After sample collection the tubes were immediately stored at $-8 \mathrm{o}^{\circ} \mathrm{C}$. All samples were afterwards transferred to a freeze drier (Labconco Freezone 12 with Labcanco Clear Drying Chamber nr.78670oo) and freeze-dried for $\sim 48$ hours. Subsequently samples were transferred to a ceramic mortar and a volume of $\sim 100 \mathrm{~mL}$ liquid $\mathrm{N}_{2}$ was added and the agar pieces were crushed using a pestle. The resulting powder was transferred to $1.2 \mathrm{~mL}$ cryo tubes (Nalgene ${ }^{\circledast}$ cryogenic tubes, Sigma-Aldrich, Zwijndrecht, Netherlands, Cat\# V4757). A amount of $~ 125 \mathrm{mg}$ agar powder was supplemented with $75 \%$ Methanol + $0.1 \%$ formic acid (Merck Methanol, Cat\# 106 oog, Merck formic acid Cat\# 100 253, Merck, Darmstadt, Germany) after solvent addition tubes were vortexed for $30 \mathrm{sec}$. . The tubes were afterwards transferred to a sonicator (Bransonic 2510, Branson Ultrasonics Corporation, Danbury, USA) and sonicated for $30 \mathrm{~min}$. After sonication the tubes were centrifuged @ 5500 rpm for 20 min (Sigma 3-16KL, Sigma Laborzentrifugen $\mathrm{GmbH}$, Germany) and the resulting supernatant was transferred to $1.2 \mathrm{~mL}$ tubes and stored at $-2 \mathrm{O}^{\circ} \mathrm{C}$ for further analysis.

\section{UHPLC-ESI-MS analysis of the extracted secondary metabolites}

One microliter of sample $(1 \mu \mathrm{l})$ was analyzed on a UHPLC system of the Ultimate 3000 series RSLC (Dionex, Sunnyvale, CA, USA) connected to a Q Exactive Hybrid Quadrupole-Orbitrap mass spectrometer (Thermo Fisher Scientific, Bremen, Germany). Separation in the UHPLC system was achieved on an Acclaim C18 column (150×2.1 mm, $2.2 \mu \mathrm{m}$, Dionex) with a flow rate of $300 \mu \mathrm{min}^{-1}$ in a binary solvent system of water and acetonitrile (hypergrade for LC MS, Merck, Darmstadt, Germany), both containing o.1 \% (v/v) formic acid (eluent additive for LC-MS, Sigma Aldrich, Steinheim, Germany). ESI source parameters were set to $3 \mathrm{kV}$ for spray voltage at a sheath gas flow of 35 and Aux gas flow of $7 \mathrm{l} / \mathrm{h}$. The voltage in the transfer capillary was set to $35 \mathrm{~V}$ at a capillary temperature of $325^{\circ} \mathrm{C}$. The samples were measured in positive 


\section{Exploring bacterial interspecific interactions for discovery of novel antimicrobial compounds}

ion mode in the mass range of $\mathrm{m} / \mathrm{z} 100-1200$ using $140,000 \mathrm{~m} / \Delta \mathrm{m}$ resolving power in the Orbitrap mass analyzer. Data were interpreted using XCALIBUR software (Thermo Fisher Scientific, Waltham, MA, USA). For statistical analysis the raw spectra were converted to mzXML format using the MS Convert feature of ProteoWizard 3.0.3750. Subsequently, data processing was carried out with R Studio 0.96.316 using the Bioconductor XCMS package. This resulting list (mz, mzmin, mzmax, rt, rtmin, rtmax, and peak intensities/areas) was saved in csv file format and uploaded into Metaboanalyst V3.0 (www.metaboanalyst.ca).

\section{Ambient mass-spectrometry analysis (LAESI-MS)}

For LAESI-MS analysis a single colony of each bacterial isolate was picked and inoculated in $20 \mathrm{~mL} \mathrm{1/10} \mathrm{o}^{\text {th }} \mathrm{TSB}$ broth and grown overnight at $24{ }^{\circ} \mathrm{C}, 220 \mathrm{rpm}$. An inoculation mix of each treatment (AD24 monoculture, AD87 monoculture and mixed strains) was prepared by diluting the bacterial isolates in $1 \mathrm{~mL}$ 10 $\mathrm{mM}$ Phosphate-buffer ( $\mathrm{pH}$ 6.5) as described above. A volume of $5 \mu$ l of monocultures or mixture was spotted in duplicates on $1 / 10^{\text {th }}$ TSBA plates at a distance of approximately $6 \mathrm{~cm}$ from each other, resulting in two single colonies per petri dish. Plates were incubated for 24 hours, 48 hours and 72 hours and subjected to LAESI-MS measurement. The bacterial colonies were cut out of the agar after 24 hours, 48 hours and 72 hours of incubation (size approximately $1 \mathrm{~cm}^{2}$ ) and transferred to a glass slide. After sample preparation the glass slide containing the samples was mounted to the sample stage. The LAESI-MS analysis was carried out on a Protea Biosciences DP-10oo LAESI system (Protea Bioscience Inc., Morgantown, WV, USA) that was coupled to a Waters model Synapt G2S (Waters Corporation, Milford, MA, USA) mass spectrometer. The LAESI system was equipped with a 2940$\mathrm{nm}$ mid-infrared laser yielding a spot size of $100 \mu \mathrm{m}$. The laser was set to fire 10 times per $\mathrm{x}$-y location (spot) at a frequency of $10 \mathrm{~Hz}$ and $100 \%$ output energy. The system was set to shoot at 100 locations per bacterial colony (in a grid of $10 \mathrm{x} 10$ positions). A syringe pump was delivering the solvent mixture of methanol/water/formic-acid (50:50:0.1\% v/v) at $2 \mathrm{~mL} / \mathrm{min}$ to a PicoTip (5cm x $100 \mu \mathrm{m}$ diameter) stainless steel nanospray emitter operating in positive ion mode at $3800 \mathrm{~V}$. The laser focus was tuned for each sample with the help of a camera. The LAESI was operated using LAESI Desktop Software V2.0.1.3 (Protea Biosciences Inc., Morgantown, WV, USA). The Time of Flight (TOF) mass analyzer of the Synapt $\mathrm{G}_{2} \mathrm{~S}$ was operated in the V-reflectron mode at a mass resolution of 18.000 to 20.000 . The source temperature was $150{ }^{\circ} \mathrm{C}$, and 
the sampling cone voltage was $30 \mathrm{~V}$. The positive ions were acquired in a mass range of 50 to $1200 \mathrm{~m} / \mathrm{z}$. The MS data were lock mass corrected post data acquisition using leucine encephalin $\left(\mathrm{C}_{28} \mathrm{H}_{37} \mathrm{~N}_{5} \mathrm{O}_{7}, \mathrm{~m} / \mathrm{z}=556.2771\right)$, which was added as internal standard to the electrospray solvent. Ions of potential interest for the generation of accurate ion maps ( $\pm 1 \mathrm{ppm})$ were identified via background subtraction using MassLynx software (Waters Corporation, Milford, MA, USA). Ion maps were created using Protea Plot V2.0.1.3 (Protea Biosciences Inc., Morgantown, WV, USA).

\section{Antibacterial assays}

The secondary metabolite extracts were used in agar disk-diffusion test in 9 $\mathrm{cm}^{2}$ petri dishes (Balouiri et al., 2016). Single colonies of each of the bacterial target organisms Escherichia coli WA321 and Staphylococcus aureus 533R4 were picked from plate and grown overnight in liquid LB broth at $37^{\circ} \mathrm{C}, 220$ rpm. Fresh LB- agar (1.5\% Merck Agar) was prepared, cooled down to $\sim 45^{\circ} \mathrm{C}$ and the target organisms were added to a final $\mathrm{OD}_{600}$ of 0.002 corresponding to approximately $6 \times 10^{\wedge} 5 \mathrm{CFU} / \mathrm{mL}$ (E.coli WA321) or $4 \times 10^{\wedge} 5 \mathrm{CFU} / \mathrm{mL}$ (S. aureus $533 \mathrm{R}_{4}$ ). To each plate $15 \mathrm{~mL}$ of the seeded agar was added and after solidification six filter papers with a diameter of $\sim 5,5 \mathrm{~mm}$ (Whatman ${ }^{\mathrm{TM}}$ filter paper Cat\# 1003-150, $6 \mu \mathrm{m}$ pore size) were placed on the top of the agar surface. A volume of $5 \mu \mathrm{l}$ of each of the extracts was added in triplicates onto the filter papers. As control a volume of $5 \mu$ l of the solvent ( $75 \%$ Methanol) was added. As positive control $5 \mu$ of appropriate antibiotic (Ampicillin 100 $\mathrm{mg} / \mathrm{mL}$ for E.coli WA321 or Tetracycline $15 \mathrm{mg} / \mathrm{mL}$ for S.aureus $533 \mathrm{R}_{4}$ ) was added onto one filter paper. As negative control filter paper with no supplemented antibiotics or secondary metabolite extracts were applied. The plates were incubated overnight at $37^{\circ} \mathrm{C}$. The next day plates were examined for visible zones of inhibition (ZOI) and digital photographs were taken. The digital images were analyzed using the AXIO VISION v4.8 imaging Software (Carl Zeiss Imaging Solutions GmbH, Germany) for surface-area determination (in $\operatorname{pixel}^{\wedge} 2$ ). All treatments were performed in six replicates.

\section{Bioassays to test the pure volatile compound 2,5-bis(1-methylethyl)- pyrazine}

The effect on growth by pure 2,5-bis(1-methylethyl)-pyrazine was tested on E. coli WA321, S. aureus 533R4 and on Rhizoctonia solani AG2.2 IIIB, Fusarium culmorum PV and Candida albicans BSMY 12 (Schmidt, 1996;Garbeva et al., 2014b; Tyc et al., 2014). The assays were performed in 12 well plates (Greiner bio-one B.V., Alphen a/d Rijn, The Netherlands, Cat\# 66518o). Stock solutions 


\section{Exploring bacterial interspecific interactions for discovery of novel antimicrobial compounds}

of $50 \mu \mathrm{l}$ pure 2,5-bis(1-methylethyl)-pyrazine (Sigma-Aldrich, Zwijndrecht, Cas\# 24294-83-5) were prepared. The model organisms E.coli WA321 and $S$. aureus $533 \mathrm{R}_{4}$ and Candida albicans BSMY212 were grown overnight either in liquid LB broth or liquid YEPD broth at $37^{\circ} \mathrm{C}, 220 \mathrm{rpm}$. Fresh LB- and YEPD agar ( $1.5 \%$ Merck Agar) was prepared and cooled down to $\sim 45{ }^{\circ} \mathrm{C}$, the target organisms were added to the liquid agar at a final $\mathrm{OD}_{600}$ of 0.002 . A volume of $1 \mathrm{~mL}$ liquid agar seeded with the test organisms was added to each well. For the test on mycelial growth fresh PD- agar (1.o \% Merck Agar) was prepared and a volume of $1 \mathrm{~mL}$ was added to each well. The fungi were added by placing a $5 \mathrm{~mm}$ diameter fungal plug of Rhizoctonia solani AG2.2 IIIB, Fusarium culmorum $\mathrm{PV}$ at the top edge of each well. To test the compound a filter paper with a diameter of $\sim 5,5 \mathrm{~mm}$ (Whatman ${ }^{\mathrm{TM}}$ filter paper Cat\# 1003-150, $6 \mu \mathrm{m}$ pore size) was placed on the agar surface at the lower edge of each compartment. A droplet of $2 \mu \mathrm{l}$ pure 2,5-bis(1-methylethyl)-pyrazine $(=1.84 \mathrm{mg})$ was added onto the filter paper. As controls $2 \mu \mathrm{l}$ of the solvent $\left(\mathrm{CHCl}_{3}\right)$ was applied (Merck Chloroform, Cat\# 102445 Merck, Darmstadt, Germany). The plates were incubated overnight at $37^{\circ} \mathrm{C}$ (E. coli, S. aureus \& C. albicans) or at $24^{\circ} \mathrm{C}$ for 4 days ( $R$. solani, F. culmorum). After incubation plates were examined for visible zones of inhibition (ZOI) or inhibition of fungal growth (mycelial extension) and digital photographs were taken. The digital images were analyzed using the AXIO VISION v4.8 imaging Software (Carl Zeiss Imaging Solutions GmbH, Germany).

Test for synergistic effects between secondary metabolite extracts and 2,5-bis(1-methylethyl)-pyrazine

To test for synergistic effects of the secondary metabolite extracts and volatile compounds agar diffusion tests with secondary metabolite extracts of the interaction Burkholderia with Paenibacillus in combination with the pure volatile compound 2,5-bis(1-methylethyl)-pyrazine were performed. The assays were carried out in 12 well plates (Greiner bio-one B.V., Alphen a/d Rijn, The Netherlands, Cat\# 665180). A stock solution of $25 \mu \mathrm{l}$ pure 2,5-bis(1methylethyl)-pyrazine was prepared in a $1.7 \mathrm{~mL}$ eppendorf tube. The model organisms E.coli $\mathrm{WA}_{321}$ and $S$. aureus $533 \mathrm{R}_{4}$ were grown overnight in liquid LB broth at $37{ }^{\circ} \mathrm{C}$, $220 \mathrm{rpm}$. Fresh LB- agar (1.5\% Merck Agar) was prepared and cooled down to $\sim 45^{\circ} \mathrm{C}$, the target organisms were added to the liquid agar at a final $\mathrm{OD}_{600}$ of 0.002 . A volume of $1 \mathrm{~mL}$ liquid agar seeded with the test organisms was added to each well. To test the secondary metabolite extracts for synergy with the pure volatile compound a filter paper with a diameter of 
$\sim 5,5 \mathrm{~mm}$ (Whatman ${ }^{\mathrm{TM}}$ filter paper Cat\# 1003-150, $6 \mu \mathrm{m}$ pore size) was placed in the middle of the agar surface in each compartment. To test for synergistic effects a droplet of $7 \mu$ l containing $2 \mu \mathrm{l}$ pure 2,5-bis(1-methylethyl)-pyrazine $(=1.84 \mathrm{mg})$ and $5 \mu \mathrm{l}$ of the secondary metabolite extracts were added onto the filter paper. As controls filter papers with $7 \mu$ lof the solvents $(5 \mu \mathrm{l}$ Merck Methanol, Cat\# 106009 and $2 \mu$ l Merck Chloroform, Cat\# 102445 Merck, Darmstadt, Germany) were applied. As positive assay control $2 \mu \mathrm{l}$ of appropriate antibiotic (Ampicillin $100 \mathrm{mg} / \mathrm{mL}$ for E.coli WA321 or Tetracycline $15 \mathrm{mg} / \mathrm{mL}$ for S.aureus $533 \mathrm{R}_{4}$ ) were applied. As negative assay control filter paper with no supplemented antibiotics or secondary metabolite extracts were applied. The plates containing the treatments were incubated overnight at $37^{\circ} \mathrm{C}$, control plates were incubated at $37^{\circ} \mathrm{C}$ in a separate incubator. After overnight incubation plates were examined for visible zones of inhibition (ZOI) and digital photographs were taken. The digital images were analyzed using the AXIO VISION v4.8 imaging Software (Carl Zeiss Imaging Solutions $\mathrm{GmbH}$, Germany) for surface-area determination (in pixel ${ }^{\wedge}{ }_{2}$ ). All treatments were performed in six replicates.

\section{Bacterial luciferase reporter assays for antimicrobial mode of action Reporter construction}

All reporter constructs were made by cloning the respective promoter, and in some cases the associated transcription factor, at the BamHI/XhoI cloning site of the medium copy number plasmid pCS26Pac (Bjarnason et al., 2003). The target fragments were amplified with PCR primers containing the appropriate restriction sites by making use of the Phusion polymerase (New England BioLabs Cat\# Mo530L). All primers used for bioreporter construction are listed in Table $\mathbf{S}_{4.3}$. The redox-cycling reporter plasmid pPHZlux-1 was constructed by amplifying the $P A 35160$ promoter and the soxR gene from the $P$. aeruginosa $\mathrm{PA} 14$ genome with the primer pair 1 and 2. The pBLAlux-2 was based on the inducible ampC promoter of $P$. protegens $\mathrm{Pf}-5$ and included also the divergently oriented the $a m p R$ regulator gene and amplified with primer pair 3 and 4 and cloned in pCS26Pac to result in pBLAlux-1. Because of the higher transcriptional induction in a $\triangle a m p D$ genetic background (data not shown), which was created by a kanamycin resistance-bearing transposon cassette, (Baba et al., 2006), a different antibiotic resistance on the plasmid was needed. Therefore a different backbone was prepared from the chloramphenicol resistance-containing pBAD33 plasmid (Guzman et al., 1995) with the primer combination 5 and 6, containing a 5'-tail with PacI restriction sites, in which the initial regulatory unit cloned in front of the luxCDABE 


\section{Exploring bacterial interspecific interactions for discovery of novel antimicrobial compounds}

operon was subcloned by digestion from the pBLAlux-1 plasmid with PacI. The pSOSlux-2 plasmid was created by introducing the colicin D promoter, subcloned from the pJAMA8-cda plasmid (Tecon et al., 2010) with primer-pair 7 and 8 in the pCS26Pac plasmid by using ClonTech In-Fusion HD Cloning Kit (\#CL 639650). Finally, a non-specific toxicity reporter was made by using a high level constitutive promoter, (Braatsch et al., 2008), which was made by annealing the complementary oligopeptide pair 9 and 10 at 1 pmole/ $\mu \mathrm{l}$ in annealing buffer (10 $\mathrm{mM}$ Tris/ $\mathrm{HCl} \mathrm{pH}=7,5,1 \mathrm{mM}$ EDTA and $50 \mathrm{mM} \mathrm{NaCl}$ ) following 5 minutes of boiling, gradual cooling to room temperature and subsequent ligation in BamHI/Xhol linearized pCS26Pac. All bacterial strains and plasmids used for bioreporter construction are listed in Table $\mathbf{S}_{\mathbf{4 . 4}}$.

\section{Bacterial luciferase reporter assays}

The reporter assays were prepared in $15 \%$ glycerol batches with a final $\mathrm{OD}_{60}$ of $\sim 0,5$. These were diluted 20 -fold in MOPS-buffered minimal medium $(8,5$ $\mathrm{mM} \mathrm{NaCl}, 18,7 \mathrm{mM} \mathrm{NH}_{4} \mathrm{Cl}, 47 \mathrm{mM}$ MOPS, o, $3 \mathrm{mM} \mathrm{Na}_{2} \mathrm{HPO}_{4} \cdot 2 \mathrm{H}_{2} \mathrm{O}, 0,3 \mathrm{mM}$ $\mathrm{KH}_{2} \mathrm{PO}_{4}, 2 \mathrm{mM}$ of $\mathrm{MgCl}_{2} ; 0.1 \mathrm{mM}$ of $\mathrm{CaCl}_{2} ; 0.2 \%$ glucose, $\mathrm{pH}=7$ ) and exposed at $1 \%$ DMSO in a final volume of $150 \mu \mathrm{l}$ in Greiner Bio-One white polystyrene 96 wells plates (Greiner bio-one B.V., Alphen a/d Rijn, The Netherlands, Cat\# 655075). Aqueous extracts of the tested bacteria were prepared by removing 4 days grown colonies of Burkholderia and Paenibacillus (monoculture) and colonies of the interaction of both bacteria (Burkholderia + Paenibacillus) from $1 / 10^{\text {th }}$ TSBA plates with a disposable inoculation loop (VWR international B.V., Amsterdam, The Netherlands Cat\# 50806-404). Scratched colonies were suspended in sterile MQ- water, homogenized by pulse- vortex for approximately $1 \mathrm{~min}$. and afterwards centrifuged for $10 \mathrm{~min}$. @ $5500 \mathrm{rpm}$. the resulting supernatant was filter sterilized ( $0.2 \mu \mathrm{M}$ Whatman filter) and stored at $-20{ }^{\circ} \mathrm{C}$ for further analysis. Reporter were exposed at $50 \%$ and taking an $E$. coli $\mathrm{DH}_{5} \alpha$ as a blank control. The volatile compound 2,5-bis(1-methylethyl)pyrazine was diluted in DMSO. The exposures of pBLAlux-2, pPHZlux-1 and pSOSlux-2 were incubated at $37^{\circ} \mathrm{C}$ at $400 \mathrm{rpm}$ for respectively 6,1 and 1 hours and a standard curve of the model compounds penicillin G, pyocyanin and mitomycin D was taken along in the same plate. Subsequently the autonomous luminescence in every well was measured during $4 \mathrm{~s}$ with a TriStar luminometer (Berthold). Samples with luminescence levels above the detection limit, i.e. average $\left(\mathrm{RLU}_{\text {blank }}\right)+3{ }^{*} \mathrm{SD}\left(\mathrm{RLU}_{\text {blank }}\right)$ were normalized for the cytotoxicity as measured by reduction of luminescence by the control reporter 
with the same plasmid backbone (pBPlux-1, or -2) measurements, analogous to (Leedjarv et al., 2006).

Mammalian luciferase reporter assays (CALUX ${ }^{\circledR}$ ) for human toxicological assessment of 2,5-bis(1-methylethyl)-pyrazine

The mammalian CALUX ${ }^{\circledR}$ reporter assays, DR-CALUX (dioxin-like activity), Nrf2-CALUX, ER $\alpha$-CALUX (estrogenic activity), AR-CALUX (androgenic activity) and p53-CALUX (genotoxicity) were conducted as describe in (van der Burg et al., 2013;van der Burg et al., 2015). The 2,5-bis(1-methylethyl)pyrazine formulation was dissolved at $0.8 \%(\mathrm{DR}), \quad 0.1 \%$ (ER $\alpha, \mathrm{AR}$ and cytotoxicity) and $1 \%$ (p53 and Nrf2) in 6 well plates in DMEM/F-12 medium (Life Technologies), while shaking at room temperature for 10 minutes and $200 \mathrm{rpm}$ at room temperature and diluted accordingly. The DMSO concentration in the wells was also adjusted to $0.8 \%(\mathrm{DR})$, o.1 \% (ER $\alpha, \mathrm{AR}$ and cytotoxicity) and $1 \%$ (p53 and $\mathrm{Nrf}_{2}$ ).

\section{Statistical analysis}

Statistical analyses on cell counts were performed with IBM SPSS Statistics 23 (IBM, Somers, NY, USA) using one-way ANOVA with post-hoc TUKEY (HSDtest). Significant differences between treatments and the controls are indicated by different letters $(\mathrm{p}<0.05)$. Statistical analysis on volatile and nonvolatile metabolites data was performed using Metaboanalyst V3.o, www.metaboanalyst.ca (Xia et al., 2015). Prior to statistical analysis data normalization was performed via log-transformation. To identify significant abundant masses ONE-WAY-ANOVA with post-hoc TUKEY test (HSD- test) was performed between the data sets. To identify important masses in the samples PLS-D analysis was performed. Masses were considered to be statistical relevant if $\mathrm{p}^{-}$values were $\leq 0.05$ and were further used for compound identification. 


\section{Exploring bacterial interspecific interactions for discovery of novel antimicrobial compounds}

\section{Results}

Effect of interspecific interaction on Burkholderia sp. AD24 and Paenibacillus sp. AD87 cell numbers

The bacterial colony forming units obtained from monocultures and interactions are summarized in Figure 4.1. Burkholderia reached the highest density in monoculture after 72 hours of incubation $\left(2.17 \times 10^{9} \mathrm{CFU} / \mathrm{mL}\right)$. The growth of Burkholderia was negatively affected when confronted with the Gram-positive Paenibacillus strain resulting in significantly lower cell counts compared to the monoculture at 48 hours and 72 hours of incubation (Figure4. 1).

In monocultures Paenibacillus reached $1.69 \times 10^{9} \mathrm{CFU} / \mathrm{mL}$ after 48 hours and $\left.7.92 \times 10^{8} \mathrm{CFU} / \mathrm{mL}\right)$ after 72 hours of incubation. During interactions the growth of Paenibacillus was negatively affected when confronted with Burkholderia only at 24 hours of incubation by reaching $2.17 \times 10^{8} \mathrm{CFU} / \mathrm{mL}$ as compared to $1.10 \times 10^{9} \mathrm{CFU} / \mathrm{mL}$ in monocultures (Figure 4.1). Overall the results revealed that growth of Burkholderia was more affected during the interspecific interaction than that of Paenibacillus.

\section{Genomic features of Burkholderia sp. AD24 and Paenibacillus sp. AD87}

The genome features (genome size, number of chromosomes and plasmids, GC content, predicted number of coding sequences and the number of rRNAs) of both bacteria are summarized in Table 4.2. Considerable differences in genome size and differences in chromosome and plasmid content were observed. The size of the two genomes differed by approximately 1.2 MB and the number of coding sequences (CDSs) varied by 1069 between Burkholderia and Paenibacillus. Overall the genome analysis revealed that the genome of Burkholderia was larger and more complex as compared to the genome of Paenibacillus. The analysis of genes probably encoding for secondary metabolites based on antiSMASH revealed a total of 14 gene clusters for Burkholderia from which 5 gene clusters belonged to the class of Bacteriocins, 3 to the class of Terpenes, 2 to Non-Ribosomal Peptides, 2 to NRPS-Hserlactones, 1 to the class of type-3 Polyketide Synthase and 1 to the class of Phosphonates. For Paenibacillus the in silico analysis revealed in total 10 gene clusters from which 2 gene clusters belonged to the class of Terpenes, 1 to Bacteriocins, 1 to Lassopeptides, 2 to the class of Lantipeptides, 1 to Non-Ribosomal Peptides, 1 to others, 1 to the class of type-3 Polyketide Synthase and one gene cluster belonging to the class of Siderophores. 


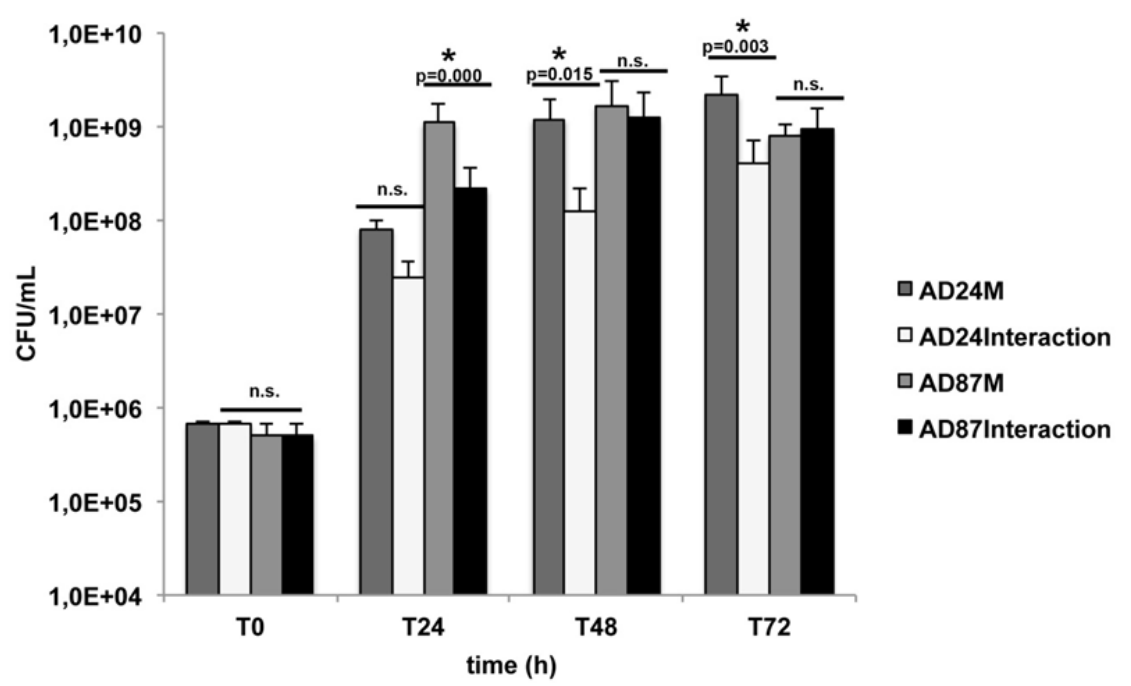

Figure 4.1: Effect of interspecific interaction on colony forming units (CFU) of Burkholderia sp. AD24 and Paenibacillus sp. AD87 grown on $1 / 10^{\text {th }}$ TSBA plates. Significant differences between treatments (pairwise combinations) and the control (monocultures) are indicated by asterisks (ONE-WAY ANOVA, post-hoc TUKEY test $\mathrm{p}<0.05$ ).

Abbreviations: AD24M: Burkholderia sp. AD24 monoculture, AD24Interaction: Burkholderia sp. AD24 in interaction with Paenibacillus sp. AD87. AD87M: Paenibacillus sp. AD87 monoculture, AD87Interaction: Paenibacillus sp. AD87 in interaction with Burkholderia sp. AD24. 


\section{Exploring bacterial interspecific interactions for discovery of novel antimicrobial compounds}

Table 4.2: Genome assembly results for Burkholderia sp. AD24 and Paenibacillus sp. AD87.

\begin{tabular}{lrr}
\hline & Burkholderia sp. AD24 & Paenibacillus sp. AD87 \\
\cline { 2 - 3 } contigs & 3 & 30 \\
bases & 8243440 & 7086713 \\
number of chromosomes & 2 & 1 \\
size chromosome 1 & 4476936 & 7086713 \\
size chromosome 2 & 3444013 & - \\
number of plasmids & 1 & - \\
size of plasmid & 322651 & - \\
CDS & 7285 & 6216 \\
rRNA & 23 & 40 \\
tmRNA & 0 & 1 \\
tRNA & 74 & 118 \\
genes & 7383 & 6375 \\
signal peptide & 742 & 547 \\
in silico detected secondary & & \\
metabolite clusters & 14 & 10 \\
(antiSMASH) & & $\mathbf{7 0 8 6 7 1 3}$ \\
\hline Total genome size (bases) & $\mathbf{8 2 4 3 4 4 0}$ &
\end{tabular}

\section{Effect of interspecific interactions on gene expression}

Transcriptome analysis revealed that the interaction between Burkholderia and Paenibacillus caused significant transcriptional changes in both bacteria as compared to the monocultures. During interaction 45 genes of Burkholderia were significantly affected after 24 hours (16 up- and 29 downregulated). At this time point no differential expression of genes was seen for Paenibacillus (Figure 4.2A). At 48 hours of incubation the expression of 38 genes (14 up- and 24 down-regulated) was significantly affected in Burkholderia whereas 531 genes were significantly differentially expressed in Paenibacillus (310 up- and 221 down-regulated) (Figure 4.2A Table 4.3, 4.4, 4.5). The highest number of differentially expressed genes was observed after 72 hours of incubation with 62 genes differentially expressed in Burkholderia (33 up- and 29 down-regulated) and 1114 genes in Paenibacillus (381 up- and 733 down-regulated) (Figure 4.2A, Table 4.3, 4.4, 4.5). Analysis based on orthologous gene categories (COG) revealed that after 48 and 72 hours most of the up-regulated genes in both bacteria belonged to the categories $S$ (function unknown), K (transcription) and NA (not assigned). 
Additionally for Paenibacillus genes belonging to the categories G (carbohydrate transport/metabolism) and for Burkholderia genes belonging to the category E (amino acid transport/metabolism) were significantly upregulated (Figure 4.2B, C). Interestingly 22 genes related to defense mechanisms (category V) were up regulated in Paenibacillus at these two time points.

At time points 48 and 72 hours most of the down regulated genes in Burkholderia belonged to the categories NA (not assigned), C (Energy production and conversion), $\mathrm{S}$ (function unknown), I (Lipid transport/metabolism) and E (amino acid transport/metabolism) (Figure 4.2B). While in Paenibacillus most of the down-regulated genes belonged to the categories $\mathrm{S}$ (function unknown), G (carbohydrate transport/metabolism) and $\mathrm{K}$ (transcription) (Figure 4.2C). 


\section{Exploring bacterial interspecific interactions for discovery of novel antimicrobial compounds}

Table 4.3: List of the top 50 differentially expressed genes (FDR $\geq 0.05$ ) in Burkholderia sp. AD24 with Paenibacillus sp. AD87 over the time points $\mathrm{t}=48 \mathrm{~h}$ to $\mathrm{t}=72 \mathrm{~h}$.

\begin{tabular}{|c|c|c|c|c|}
\hline Sequence ID & Gene description & $\begin{array}{c}\text { fold change with } \\
\text { Paenibacillus sp. AD87 }\end{array}$ & $\begin{array}{l}\text { FDR } \\
\text { value }\end{array}$ & possible function \\
\hline $\begin{array}{l}\text { bAD24_p01665 } \\
\end{array}$ & Type IV secretion system protein virB1 & 3,94 & 0,00 & Cell wall/membrane/envelope biogenesis \\
\hline bAD24_1112395 & Aminopeptidase $\mathrm{N}$ & 3,88 & 0,00 & Amino acid transport and metabolism \\
\hline bAD24_p01670 & Flagellar biosynthetic protein FliP & 3,46 & 0,00 & Intracellular trafficking, secretion, and vesicular transport \\
\hline bAD24_1108090 & Transcriptional activator protein NhaR & 3,12 & 0,00 & Transcription \\
\hline bAD24_II11090 & hypothetical protein & 3,07 & 0,03 & Function unknown \\
\hline bAD24_p01610 & hypothetical protein & 2,92 & 0,05 & Function unknown \\
\hline bAD24_1105560 & hypothetical protein & 2,89 & 0,00 & Cell wall/membrane/envelope biogenesis \\
\hline bAD24_1108070 & putative lipoprotein YiaD & 2,69 & 0,01 & Cell motility \\
\hline bAD24_1108565 & hypothetical protein & 2,57 & 0,01 & Function unknown \\
\hline bAD24_II15035 & 23 S ribosomal RNA & 2,56 & 0,00 & NA \\
\hline bAD24_113295 & 23 S ribosomal RNA & 2,56 & 0,00 & NA \\
\hline bAD24_1103345 & $23 \mathrm{~S}$ ribosomal RNA & 2,54 & 0,01 & NA \\
\hline bAD24_100140 & Adenylyl-sulfate kinase & 2,47 & 0,00 & Amino acid transport and metabolism \\
\hline bAD24_1103425 & hypothetical protein & 2,47 & 0,01 & Function unknown \\
\hline bAD24_105570 & $23 \mathrm{~S}$ ribosomal RNA & 2,42 & 0,01 & NA \\
\hline bAD24_1108075 & hypothetical protein & 2,38 & 0,01 & Function unknown \\
\hline bAD24_p01560 & hypothetical protein & 2,36 & 0,02 & Function unknown \\
\hline bAD24_II13810 & 23 S ribosomal RNA & 2,30 & 0,01 & NA \\
\hline bAD24_100825 & Transcriptional activator protein LasR & 2,29 & 0,01 & Transcription \\
\hline bAD24_p01565 & hypothetical protein & 2,29 & 0,01 & Mobilome: prophages, transposons \\
\hline bAD24_1106980 & Carboxymethylenebutenolidase & 2,27 & 0,04 & Secondary metabolites biosynthesis, transport and catabolism \\
\hline bAD24_1102950 & $16 \mathrm{~S}$ ribosomal RNA & 2,24 & 0,02 & NA \\
\hline bAD24_II10295 & hypothetical protein & 2,23 & 0,02 & Function unknown \\
\hline bAD24_112375 & 23 S ribosomal RNA & 2,21 & 0,02 & NA \\
\hline bAD24_105585 & $16 \mathrm{~S}$ ribosomal RNA & 2,19 & 0,02 & NA \\
\hline bAD24_II12400 & Disulfide-bond oxidoreductase YfcG & 2,17 & 0,03 & Posttranslational modification, protein turnover, chaperones \\
\hline bAD24_p01570 & Cyclic di-GMP phosphodiesterase response regulator RpfG & 2,15 & 0,02 & Signal transduction mechanisms \\
\hline bAD24_p01660 & hypothetical protein & 2,14 & 0,03 & Posttranslational modification, protein turnover, chaperones \\
\hline bAD24_1108380 & HTH-type transcriptional regulator PgrR & 2,12 & 0,02 & Transcription \\
\hline bAD24_1102935 & 23 S ribosomal RNA & 2,09 & 0,03 & NA \\
\hline bAD24_II15050 & $16 \mathrm{~S}$ ribosomal RNA & 2,07 & 0,03 & NA \\
\hline bAD24_1100020 & $16 \mathrm{~S}$ ribosomal RNA & 2,01 & 0,04 & NA \\
\hline bAD24_II13825 & $16 \mathrm{~S}$ ribosomal RNA & 1,93 & 0,05 & NA \\
\hline bAD24_1106535 & Bicarbonate transport ATP-binding protein $\mathrm{CmpD}$ & $-6,83$ & 0,02 & Inorganic ion transport and metabolism \\
\hline bAD24_104185 & putative acetoacetate decarboxylase & $-6,74$ & 0,03 & Secondary metabolites biosynthesis, transport and catabolism \\
\hline bAD24_107975 & Hydroxymethylglutaryl-CoA lyase YngG & $-6,74$ & 0,03 & Amino acid transport and metabolism \\
\hline bAD24_118450 & hypothetical protein & $-6,53$ & 0,04 & Lipid transport and metabolism \\
\hline bAD24_II14900 & HTH-type transcriptional regulator $M c b R$ & $-6,36$ & 0,05 & Transcription \\
\hline bAD24_101620 & Glycerate dehydrogenase & $-4,31$ & 0,00 & Energy production and conversion \\
\hline bAD24_1101060 & $\begin{array}{l}\text { Alpha-D-ribose 1-methylphosphonate 5-triphosphate } \\
\text { synthase subunit PhnH }\end{array}$ & $-3,86$ & 0,02 & Inorganic ion transport and metabolism \\
\hline bAD24_II11545 & hypothetical protein & $-3,86$ & 0,02 & Function unknown \\
\hline bAD24_1101170 & Mycothiol acetyltransferase & $-3,79$ & 0,03 & General function prediction only \\
\hline bAD24_1104610 & Glycine cleavage system transcriptional activator & $-3,79$ & 0,03 & Transcription \\
\hline bAD24_1103270 & hypothetical protein & $-3,72$ & 0,00 & Function unknown \\
\hline bAD24_11000005 & $23 \mathrm{~S}$ ribosomal RNA (partial) & $-3,61$ & 0,00 & NA \\
\hline bAD24_105585 & $16 \mathrm{~S}$ ribosomal RNA & $-3,57$ & 0,00 & NA \\
\hline bAD24_II11260 & hypothetical protein & $-3,53$ & 0,00 & Function unknown \\
\hline bAD24_1107555 & $\begin{array}{l}\text { tRNA 5-methylaminomethyl-2-thiouridine biosynthesis } \\
\text { bifunctional protein MnmC }\end{array}$ & $-3,47$ & 0,01 & General function prediction only \\
\hline bAD24_105445 & hypothetical protein & $-3,21$ & 0,00 & Function unknown \\
\hline bAD24_1102950 & $16 \mathrm{~S}$ ribosomal RNA & $-3,12$ & 0,00 & NA \\
\hline bAD24_II14670 & Cytochrome bd-I ubiquinol oxidase subunit 1 & $-3,11$ & 0,00 & Energy production and conversion \\
\hline
\end{tabular}




\section{Differentially expressed genes related to secondary metabolite production}

In Burkholderia the gene bAD24_IIo698o (Carboxymethylenebutenolidase) (secondary metabolite biosynthesis) was differentially expressed at 48 and 72 hours. The gene was 2.27 fold up-regulated as compared to the control (Table 4.3). In Paenibacillus the gene gpAD87_o189o (Dienelactone hydrolase) was 3.9 fold up-regulated compared to the monoculture at 48 hours of incubation. At 72 hours in total 3 genes were highly expressed namely: gpAD87_1379o (Dienelactone hydrolase), gpAD87_02615 (Imidazolonepropionase), gpAD87_0189o (Dienelactone hydrolase). These genes were 3.4 fold and 1.9 fold respectively higher expressed during interaction as compared to the monoculture (Table 4.4).

\section{Differentially expressed genes related to signal transduction}

During the interaction of Burkholderia and Paenibacillus several genes related to the signal transduction systems (category $\mathrm{T}$ ) were affected. In Burkholderia the gene bAD24_po157o (Cyclic di-GMP phosphodiesterase response regulator RpfG) was 2.15 fold higher expressed as compared to the control (Table 4.3). Interestingly this gene was found on a mobile genetic element. In Paenibacillus 57 genes related to signal transduction were affected, at 48 hours 22 genes were up regulated and 35 genes down regulated. The genes gpAD87_21325 (Transcriptional regulatory protein LiaR) and gpAD87_2370o (HPr-like protein $\mathrm{Crh}$ ) were the most affected with 6.84 up and 3.73 fold change down regulated respectively (Table 4.4, 4.5). At time point $t=72 \mathrm{~h} 30$ genes were up regulated and 41 genes were down-regulated (Figure 4.2A) from which gene gpAD87_26840 (Methyl-accepting chemotaxis protein MсрB) and gpAD87_07465 (Low-molecular weight protein-tyrosinephosphatase YfkJ) were the most affected with 7.41 up and 8.46 down regulated, respectively (Table 4.4, 4.5). 


\section{Exploring bacterial interspecific interactions for discovery of novel antimicrobial compounds}

Table 4.4: Top 50 up-regulated genes (FDR $\geq 0.05)$ in Paenibacillus sp. AD87 during interaction with Burkholderia sp. AD24 over the time points $t=48 \mathrm{~h}$ to $\mathrm{t}=72 \mathrm{~h}$.

\begin{tabular}{|c|c|c|c|c|}
\hline Sequence ID & Gene description & $\begin{array}{c}\text { fold change with } \\
\text { Burkholderia sp. AD24 }\end{array}$ & $\begin{array}{r}\text { FDR } \\
\text { value }\end{array}$ & possible function \\
\hline gpAD87_28110 & Vancomycin B-type resistance protein VanW & 9,36 & 0,00 & Defense mechanisms \\
\hline gpAD87_16925 & L-erythro-3,5-diaminohexanoate dehydrogenase & 7,66 & 0,00 & Cell wall/membrane/envelope biogenesis \\
\hline gpAD87_27745 & UDP-N-acetylglucosamine 1-carboxyvinyltransferase 1 & 7,47 & 0,00 & Cell wall/membrane/envelope biogenesis \\
\hline gpAD87_26840 & Methyl-accepting chemotaxis protein McpB & 7,41 & 0,01 & Signal transduction mechanisms \\
\hline gpAD87_09100 & Pesticidal crystal protein cry22Aa & 7,37 & 0,00 & Mobilome: prophages, transposons \\
\hline gpAD87_01885 & $\mathrm{KHG} / \mathrm{KDPG}$ aldolase & 7,36 & 0,00 & Carbohydrate transport and metabolism \\
\hline gpAD87_18840 & putative $\mathrm{ABC}$ transporter permease & 7,36 & 0,00 & Defense mechanisms \\
\hline gpAD87_19800 & Autoinducer 2 import ATP-binding protein LsrA & 7,13 & 0,00 & General function prediction only \\
\hline gpAD87_20500 & hypothetical protein & 7,13 & 0,00 & Function unknown \\
\hline gpAD87_26670 & 4-carboxy-2-hydroxymuconate-6-semialdehyde dehydrogenase & 6,99 & 0,01 & General function prediction only \\
\hline gpAD87_07910 & Esterase EstB & 6,84 & 0,01 & Defense mechanisms \\
\hline gpAD87_14330 & NADH oxidase & 6,84 & 0,01 & Energy production and conversion \\
\hline gpAD87_17325 & Magnesium and cobalt efflux protein CorC & 6,84 & 0,01 & General function prediction only \\
\hline gpAD87_21325 & Transcriptional regulatory protein LiaR & 6,84 & 0,01 & Signal transduction mechanisms \\
\hline gpAD87_24045 & Potassium-transporting ATPase $\mathrm{C}$ chain & 6,73 & 0,00 & Inorganic ion transport and metabolism \\
\hline gpAD87_18375 & hypothetical protein & 6,67 & 0,01 & Function unknown \\
\hline gpAD87_20865 & hypothetical protein & 6,67 & 0,01 & Function unknown \\
\hline gpAD87_18365 & hypothetical protein & 6,50 & 0,00 & Function unknown \\
\hline gpAD87_13865 & hypothetical protein & 6,48 & 0,03 & General function prediction only \\
\hline gpAD87_19825 & Urease subunit beta & 6,48 & 0,03 & Amino acid transport and metabolism \\
\hline gpAD87_16035 & Arabinogalactan endo-beta-1,4-galactanase & 6,41 & 0,00 & Carbohydrate transport and metabolism \\
\hline gpAD87_00220 & putative oxidoreductase YcjS & 6,26 & 0,04 & General function prediction only \\
\hline gpAD87_12085 & hypothetical protein & 6,26 & 0,04 & Translation, ribosomal structure and biogenesis \\
\hline gpAD87_13630 & Response regulator protein VraR & 6,26 & 0,04 & Transcription \\
\hline gpAD87_25705 & 3-oxoacyl-[acyl-carrier-protein] reductase FabG & 6,26 & 0,04 & General function prediction only \\
\hline gpAD87_25320 & hypothetical protein & 6,25 & 0,00 & Function unknown \\
\hline gpAD87_16040 & Inner membrane $A B C$ transporter permease protein $Y c j P$ & 6,16 & 0,00 & Carbohydrate transport and metabolism \\
\hline gpAD87_18370 & RNA-splicing ligase RtcB & 5,85 & 0,00 & Translation, ribosomal structure and biogenesis \\
\hline gpAD87_23460 & 23 S ribosomal RNA (partial) & 5,74 & 0,00 & NA \\
\hline gpAD87_16030 & Beta-galactosidase BglY & 5,67 & 0,00 & Carbohydrate transport and metabolism \\
\hline gpAD87_16020 & hypothetical protein & 5,64 & 0,00 & Translation, ribosomal structure and biogenesis \\
\hline gpAD87_06185 & Chorismate synthase & 5,58 & 0,00 & Amino acid transport and metabolism \\
\hline gpAD87_06685 & Sensory transduction protein reg $X 3$ & 5,49 & 0,00 & Signal transduction mechanisms \\
\hline gpAD87_12310 & $\begin{array}{l}\text { 2-hydroxy-6-oxononadienedioate/2-hydroxy-6- } \\
\text { oxononatrienedioate hydrolase }\end{array}$ & 5,48 & 0,00 & Coenzyme transport and metabolism \\
\hline gpAD87_05105 & putative MFS-type transporter YhjX & 5,35 & 0,00 & Inorganic ion transport and metabolism \\
\hline gpAD87_30715 & hypothetical protein & 5,31 & 0,00 & Function unknown \\
\hline gpAD87_12650 & Oleandomycin glycosyltransferase & 5,30 & 0,00 & Carbohydrate transport and metabolism \\
\hline gpAD87_22925 & Inner membrane protein YqjA & 5,27 & 0,00 & Function unknown \\
\hline gpAD87_21995 & Cyclopentanol dehydrogenase & 5,22 & 0,00 & Lipid transport and metabolism \\
\hline gpAD87_06680 & Alkaline phosphatase synthesis sensor protein PhoR & 5,14 & 0,00 & Signal transduction mechanisms \\
\hline gpAD87_00465 & 3-oxoacyl-[acyl-carrier-protein] reductase FabG & 5,05 & 0,00 & Lipid transport and metabolism \\
\hline gpAD87_02880 & $\mathrm{K}(+) / \mathrm{H}(+)$ antiporter subunit KhtT & 4,97 & 0,00 & Inorganic ion transport and metabolism \\
\hline gpAD87_11435 & putative metallo-hydrolase YfIN & 4,97 & 0,00 & General function prediction only \\
\hline gpAD87_16015 & hypothetical protein & 4,93 & 0,00 & Function unknown \\
\hline gpAD87_01305 & hypothetical protein & 4,92 & 0,00 & Function unknown \\
\hline gpAD87_04025 & GTPase Era & 4,88 & 0,00 & Translation, ribosomal structure and biogenesis \\
\hline gpAD87_10330 & Arabinose operon regulatory protein & 4,88 & 0,00 & Transcription \\
\hline gpAD87_13790 & Acetyl esterase Axe7A & 3,49 & 0,00 & Secondary metabolites biosynthesis, transport and catabolism \\
\hline gpAD87_02615 & Imidazolonepropionase & 1,97 & 0,03 & Secondary metabolites biosynthesis, transport and catabolism \\
\hline gpAD87_01890 & Homoserine O-acetyltransferase & 1,96 & 0,03 & Secondary metabolites biosynthesis, transport and catabolism \\
\hline
\end{tabular}




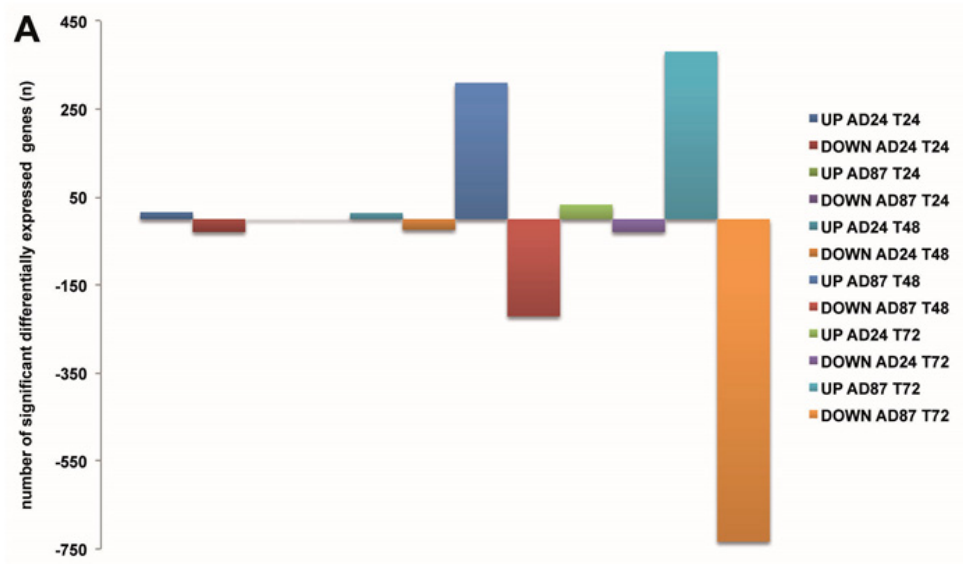

B
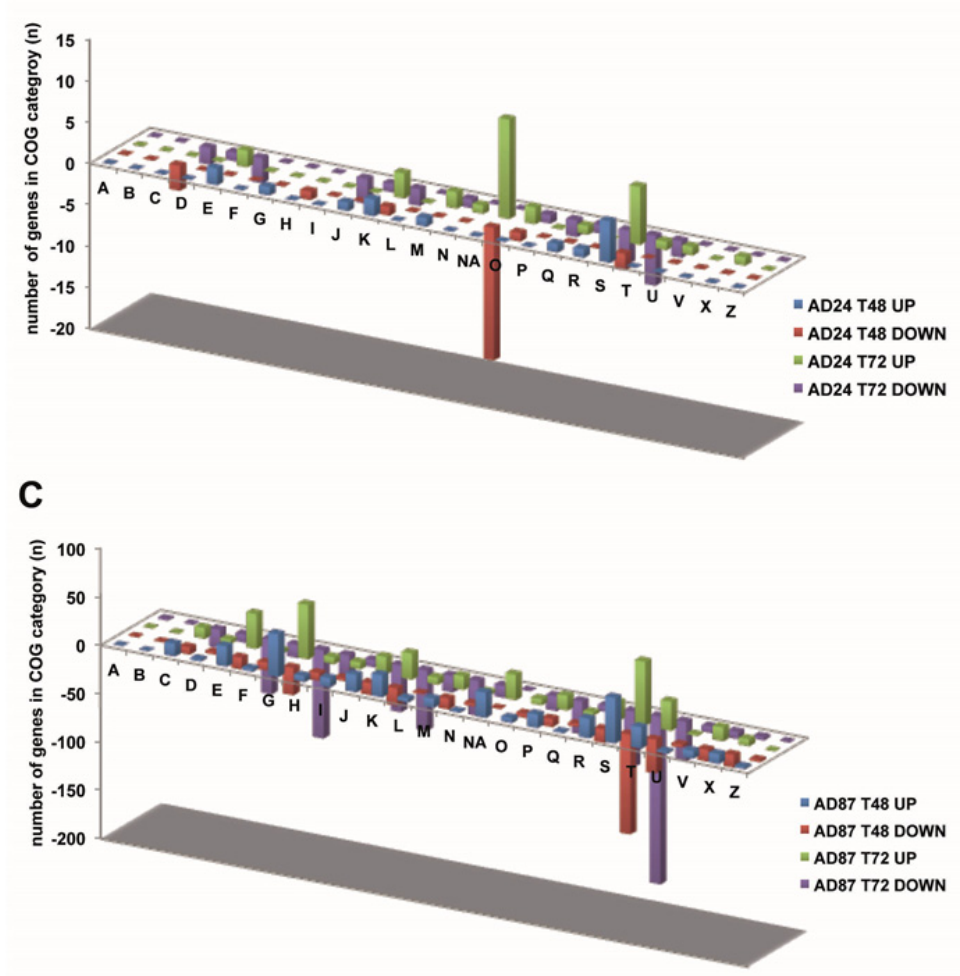

Figure 4.2: Overview of the transcriptome analysis outcome for Burkholderia sp. AD24 and Paenibacillus sp. AD87 during co-culturing on $1 / 10^{\text {th }}$ TSB agar. (A) overview of the number ( $\mathrm{n}$ ) of significantly (FDR <0.05) differently expressed genes over the time points $\mathrm{T}_{24} \mathrm{~h}-\mathrm{T}_{72} \mathrm{~h}$. (B and $\mathbf{C}$ ) Overview of the COG classification of differentially expressed genes during interspecific 


\section{Exploring bacterial interspecific interactions for discovery of novel antimicrobial compounds}

interaction between Burkholderia sp. AD24 and Paenibacillus sp. AD87 over the time points $\mathrm{T}_{24}(\mathrm{t}=24 \mathrm{~h})$ till $\mathrm{T}_{72}(\mathrm{t}=72 \mathrm{~h})$ relative to the monoculture condition. Differentially expressed genes based on COG classification for b: Burkholderia sp. AD24 in interaction with Paenibacillus sp. AD87 and c: Paenibacillus in interaction with Burkholderia sp. AD24. The one-letter codes represent the following functional categories: A: RNA processing and modification, B: Chromatin structure and dynamics, C: energy production and conversion; D: cell cycle control, cell division, chromosome partitioning; E: amino acid transport and metabolism; F: nucleotide transport and metabolism; G: carbohydrate transport and metabolism; H: coenzyme transport and metabolism; I: lipid transport and metabolism; J: translation, ribosomal structure and biogenesis; K: transcription; $\mathrm{L}$ : replication, recombination and repair; $\mathrm{M}$ : cell wall/membrane/envelope biogenesis; $\mathrm{N}$ : cell motility; NA: not assigned; $\mathrm{O}$ : posttranslational modification, protein turnover; chaperones; P: inorganic ion transport and metabolism; Q: secondary metabolites biosynthesis, transport and catabolism; R: general function prediction only; $\mathrm{S}$ : function unknown; $\mathrm{T}$ : signal transduction mechanisms; U: intracellular trafficking, secretion, and vesicular transport; V: defense mechanisms X: mobilome: prophages, transposons.

\section{Differentially expressed genes related to defense mechanisms}

In total 22 genes belonging to defense mechanisms were affected in Paenibacillus after 48 hours ( 8 up-regulated, 14 down-regulated) and 19 genes were affected at 72 hours (12 up-regulated and 7 down-regulated) (Figure 4.2B, C). At both time points the most affected genes in Paenibacillus were gpAD87_28110 (Vancomycin B-type resistance protein (VanW) and gpAD87_18840 (putative ABC transporter permease) (Table 4.4).

The two most down-regulated genes at time point $\mathrm{t}=48 \mathrm{~h}$ were gene gpAD87_12115 (Multidrug resistance protein (YkkD)) and gene gpAD87_09055 (Multidrug resistance protein (NorM)) with 6.73 and 4.67 fold changes respectively (Table 4.5). At $\mathrm{t}=72 \mathrm{~h}$ the genes gpAD87_14640 (Putative penicillin-binding protein) and gpAD87_06980 (RutC family protein) were down regulated with fold changes of 6.94 and 5.96 respectively (Table 4.5). 
Table 4.5: Top 50 down-regulated genes (FDR $\geq 0.05$ ) in Paenibacillus sp. AD87 during interaction with Burkholderia sp. $\mathrm{AD}_{24}$ over the time points $\mathrm{t}=48 \mathrm{~h}$ to $\mathrm{t}=72 \mathrm{~h}$.

\begin{tabular}{|c|c|c|c|c|}
\hline Sequence ID & Gene description & $\begin{array}{c}\text { fold change with } \\
\text { Burkholderia sp. AD24 }\end{array}$ & $\begin{array}{c}\text { FDR } \\
\text { value }\end{array}$ & possible function \\
\hline gpAD87_04615 & hypothetical protein & $-10,27$ & 0,00 & Function unknown \\
\hline gpAD87_07985 & hypothetical protein & $-9,88$ & 0,00 & Function unknown \\
\hline gpAD87_30040 & Putative monooxygenase $\mathrm{YcnE}$ & $-9,81$ & 0,00 & Energy production and conversion \\
\hline gpAD87_09355 & putative methyltransferase $\mathrm{Ycg} J$ & $-9,73$ & 0,00 & Coenzyme transport and metabolism \\
\hline gpAD87_14585 & Acetyltransferase YpeA & $-9,56$ & 0,00 & Translation, ribosomal structure and biogenesis \\
\hline gpAD87_04655 & hypothetical protein & $-9,38$ & 0,00 & Function unknown \\
\hline gpAD87_29715 & Inositol-1-monophosphatase & $-9,23$ & 0,00 & Carbohydrate transport and metabolism \\
\hline gPAD87_17060 & hypothetical protein & $-9,20$ & 0,00 & Function unknown \\
\hline gPAD87_14605 & Acetyltransferase YpeA & $-9,01$ & 0,00 & Translation, ribosomal structure and biogenesis \\
\hline gPAD87_22100 & Alanine--tRNA ligase & $-8,98$ & 0,00 & Translation, ribosomal structure and biogenesis \\
\hline gpAD87_31275 & Endonuclease YhcR & $-8,92$ & 0,00 & RNA processing and modification \\
\hline gPAD87_12550 & hypothetical protein & $-8,86$ & 0,00 & Function unknown \\
\hline gpAD87_23185 & Serine/threonine-protein kinase AfsK & $-8,73$ & 0,00 & Function unknown \\
\hline gpAD87_14475 & hypothetical protein & $-8,72$ & 0,00 & Function unknown \\
\hline gpAD87_14595 & hypothetical protein & $-8,71$ & 0,00 & Function unknown \\
\hline gpAD87_14750 & Putative mutator protein MutT4 & $-8,71$ & 0,00 & Nucleotide transport and metabolism \\
\hline gPAD87_20950 & Proline dehydrogenase 1 & $-8,63$ & 0,00 & Amino acid transport and metabolism \\
\hline gpAD87_28445 & Elongation factor $\mathrm{G}$ & $-8,63$ & 0,00 & Translation, ribosomal structure and biogenesis \\
\hline gpAD87_27985 & CDP-glucose 4,6-dehydratase & $-8,58$ & 0,00 & Cell wall/membrane/envelope biogenesis \\
\hline gpAD87_08715 & Monomeric sarcosine oxidase & $-8,56$ & 0,00 & Amino acid transport and metabolism \\
\hline gpAD87_31365 & hypothetical protein & $-8,56$ & 0,00 & Amino acid transport and metabolism \\
\hline gpAD87_07465 & Low molecular weight protein-tyrosine-phosphatase YfkJ & $-8,46$ & 0,00 & Signal transduction mechanisms \\
\hline gPAD87_12005 & Aldose 1-epimerase & $-8,44$ & 0,00 & Carbohydrate transport and metabolism \\
\hline gPAD87_16995 & Chaperone protein $\mathrm{HtpG}$ & $-8,39$ & 0,00 & Posttranslational modification, protein turnover, chaperones \\
\hline gpAD87_24970 & Single-stranded DNA-binding protein A & $-8,37$ & 0,00 & Replication, recombination and repair \\
\hline gpAD87_27970 & $\mathrm{N}$-acylneuraminate cytidylyltransferase & $-8,37$ & 0,00 & Cell wall/membrane/envelope biogenesis \\
\hline gPAD87_09250 & hypothetical protein & $-8,35$ & 0,00 & Function unknown \\
\hline gPAD87_15590 & Ribosome biogenesis GTPase A & $-8,35$ & 0,00 & Translation, ribosomal structure and biogenesis \\
\hline gpAD87_00840 & Glutathione transport system permease protein GsiC & $-8,33$ & 0,00 & Amino acid transport and metabolism \\
\hline gPAD87_29710 & hypothetical protein & $-8,28$ & 0,00 & Function unknown \\
\hline gpAD87_19650 & putative endonuclease 4 & $-8,23$ & 0,00 & Replication, recombination and repair \\
\hline gPAD87_00905 & Oligopeptide transport ATP-binding protein OppD & $-8,21$ & 0,00 & Amino acid transport and metabolism \\
\hline gPAD87_09130 & Sulfoacetaldehyde reductase & $-8,18$ & 0,00 & General function prediction only \\
\hline gpAD87_05250 & hypothetical protein & $-8,10$ & 0,00 & Function unknown \\
\hline gpAD87_31865 & hypothetical protein & $-8,10$ & 0,00 & General function prediction only \\
\hline gPAD87_10400 & hypothetical protein & $-8,08$ & 0,00 & Function unknown \\
\hline gPAD87_28520 & $50 \mathrm{~S}$ ribosomal protein $\mathrm{L} 5$ & $-8,05$ & 0,00 & Translation, ribosomal structure and biogenesis \\
\hline gpAD87_00470 & $\mathrm{Fe} / \mathrm{S}$ biogenesis protein NfuA & $-7,99$ & 0,00 & Posttranslational modification, protein turnover, chaperones \\
\hline gpAD87_29875 & hypothetical protein & $-7,99$ & 0,00 & Function unknown \\
\hline gpAD87_11325 & Sensor histidine kinase YehU & $-7,91$ & 0,00 & Signal transduction mechanisms \\
\hline gPAD87_14755 & hypothetical protein & $-7,91$ & 0,00 & Function unknown \\
\hline gPAD87_14315 & hypothetical protein & $-7,87$ & 0,00 & Function unknown \\
\hline gPAD87_22410 & putative metallo-hydrolase & $-7,78$ & 0,00 & General function prediction only \\
\hline gPAD87_23025 & hypothetical protein & $-7,78$ & 0,00 & Function unknown \\
\hline gPAD87_23175 & hypothetical protein & $-7,78$ & 0,00 & Function unknown \\
\hline gpAD87_07425 & Sulfur carrier protein ThiS & $-7,71$ & 0,00 & Coenzyme transport and metabolism \\
\hline gPAD87_00725 & CheY-P phosphatase CheX & $-7,67$ & 0,00 & Cell motility \\
\hline gpAD87_08830 & putative response regulatory protein & $-7,67$ & 0,00 & Signal transduction mechanisms \\
\hline gPAD87_14640 & Putative penicillin-binding protein $\mathrm{PbpX}$ & $-6,94$ & 0,01 & Defense mechanisms \\
\hline gpAD87_06980 & RutC family protein & $-5,96$ & 0,00 & Defense mechanisms \\
\hline
\end{tabular}




\section{Exploring bacterial interspecific interactions for discovery of novel antimicrobial compounds}

\section{Q-PCR results}

The results from the transcriptome analysis were confirmed by performing qRT-PCR. Comparison of the fold changes in gene expression of gene bAD24_10391 in Burkholderia and of gene gpAD87_304 in Paenibacillus confirmed that the expression of the targeted genes were approximately at the same level as in the transcriptome analysis (Table $\mathbf{S}_{\mathbf{4 . 5}}$ ).

\section{Effect of interspecific interaction on secondary metabolite production Soluble metabolites}

Metabolome analysis performed on extracts of monocultures and interactions of Burkholderia and Paenibacillus revealed that the metabolite composition of the monocultures differed from that of the mixtures (Figure 4.3A). Clear separations of metabolite composition between controls, monocultures and interactions were obtained in PLS-DA score plots (Figure 4.3A). One of the compounds observed in a higher concentration during interaction was identified as a Pederin-like compound $\left(\mathrm{C}_{25} \mathrm{H}_{45} \mathrm{NO}_{9}, \mathrm{~m} / \mathrm{z}=504.316\right)\left[\mathrm{M}+\mathrm{H}^{+}\right]$ (Figure S4.6).

\section{Volatile metabolites}

The comparison of volatile organic compounds emitted by the bacteria revealed that the volatile composition of the monocultures differed significantly from that of the mixtures (Figure 4.3B). Clear separations between the monocultures, controls and the interaction were obtained in PLSDA score plots (Figure 4.3B). The analysis revealed 22 volatile organic compounds produced by bacteria that were not detected in the noninoculated controls (Table 4.6). Of these volatile organic compounds 17 could be tentatively identified and categorized in 6 different chemical classes (Alkenes, Benzoids, Sulfides, Terpenes, Furans, and Pyrazines). However, five compounds could not be assigned with certainty to a volatile organic compound. The most prominent headspace volatile organic compounds were sulfur-containing compounds (such as dimethyl disulfide, dimethyl trisulfide etc.). Two sulfur compounds dimethyl disulfide $\left(\mathrm{C}_{2} \mathrm{H}_{6} \mathrm{~S}_{2}\right)$ and dimethyl trisulfide $\left(\mathrm{C}_{2} \mathrm{H}_{6} \mathrm{~S}_{3}\right)$ were produced by both Burkholderia and Paenibacillus. Interestingly two compounds produced by the monoculture of Burkholderia (S-Methyl methanethiosulfonate and unknown compound 4) were not detected during the interaction with Paenibacillus (Table 4.6). One volatile organic compound was produced in very high concentration during the interaction of the two bacteria. This compound was identified as 2,5-bis(1methylethyl)-pyrazine $\left(\mathrm{C}_{10} \mathrm{H}_{16} \mathrm{~N}_{2}, m / z=164.247, \mathrm{RT}=19.7\right)$ and is most probably 
produced by Burkholderia (Figure S4.7, Table 4.6). For confirmation of the structure and for bioassays, the 2,5-bis(1-methylethyl)-pyrazine was commercially synthesized.
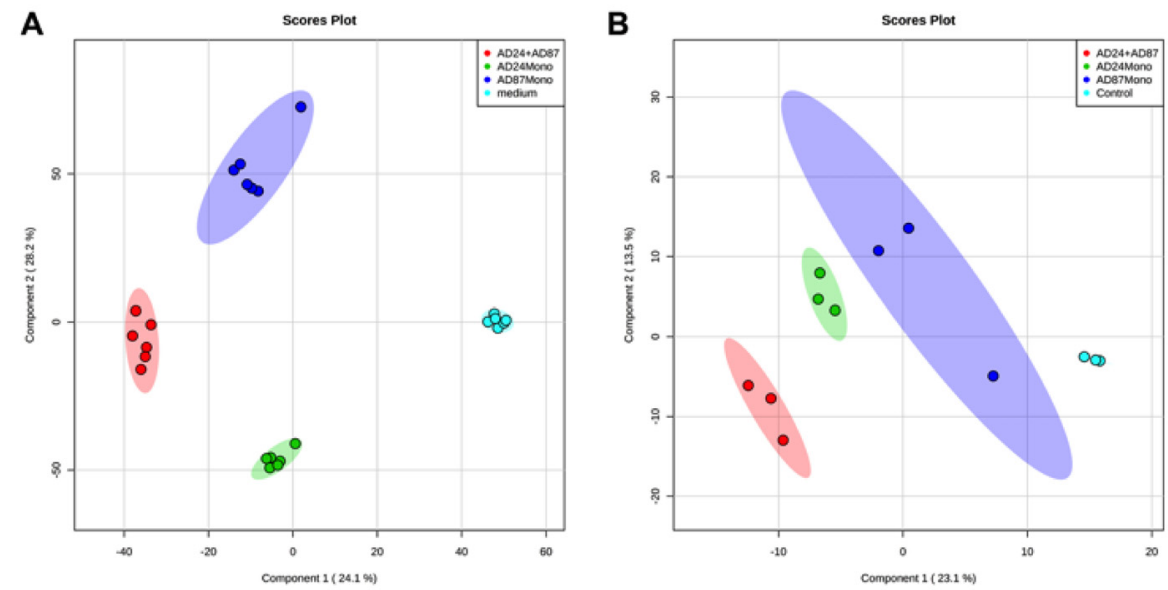

Figure 4.3: PLSDA plots of metabolomics data of monocultures and interactions of Burkholderia sp. AD24 and Paenibacillus sp. AD87 (A) PLSDA $2 \mathrm{D}$ - plots of the analyzed LC-MS data of soluble compounds after three days of incubation) (B) PLSDA 2D- plots of GC-MS data of volatiles emitted after three days of inoculation.

\section{LAESI- mass spectrometry (ambient imaging mass spectrometry)}

The LAESI-DP10oo system was used to visualize the production of secondary metabolites by living bacterial colonies. With the LAESI-mass spectrometry system we confirmed the production of the two identified compounds 2,5bis(1-methylethyl)-pyrazine $(m / z=164.247)$ and the unknown Pederin-like compound $\left(\mathrm{m} / \mathrm{z}=526.298\left[\mathrm{M}+\mathrm{Na}^{+}\right]\right)$in the micro colonies of Burkholderia and Paenibacillus (Figure 4.4A, B). 


\section{Exploring bacterial interspecific interactions for discovery of novel antimicrobial compounds}

Table 4.6: Tentatively identified volatile organic compounds (VOCs) produced by a Burkholderia and Paenibacillus strain in mono- and co-culture on $1 / 10^{\text {th }}$ TSB agar.

\begin{tabular}{|c|c|c|c|c|c|c|c|c|}
\hline \multirow[b]{2}{*}{$\#$} & \multirow[b]{2}{*}{ Compound name } & \multirow[b]{2}{*}{$\mathbf{R} \mathbf{T}^{*}$} & \multirow[b]{2}{*}{$\mathrm{ELRI}^{* *}$} & \multirow[b]{2}{*}{ p-value ${ }^{* * *}$} & \multirow[b]{2}{*}{ chemical class } & \multicolumn{3}{|c|}{ Detected in treatment } \\
\hline & & & & & & Burk & Paen & $\begin{array}{c}\text { MIX } \\
\text { Burk+Paen }\end{array}$ \\
\hline 1 & 1,3-butadiene, 2-methyl- & 2,11 & 525 & 0,018 & Alkenes & $\bar{x}$ & $\bar{x}$ & $\mathbf{X}$ \\
\hline 2 & 2-methylfuran & 2,53 & 586 & 0,030 & Furan & $\mathbf{x}$ & $\mathbf{x}$ & $\mathbf{x}$ \\
\hline 3 & dimethyl disulfide & 4,20 & 741 & 0,001 & Sulfides & $\mathbf{x}$ & $\mathbf{x}$ & $\mathbf{x}$ \\
\hline 4 & toluene & 4,63 & 762 & 0,000 & Benzenoids & $\mathbf{x}$ & $\mathbf{x}$ & $\mathbf{x}$ \\
\hline 5 & unknown compound 1 & 5,25 & 786 & 0,000 & - & $\mathbf{x}$ & $\mathbf{x}$ & $\mathbf{x}$ \\
\hline 6 & 1,3-dithiethane & 5,44 & 793 & 0,001 & Sulfides & $x$ & & $\mathbf{x}$ \\
\hline 7 & 2,4 dithiapentane & 7,96 & 887 & 0,009 & Sulfides & $\mathbf{x}$ & & $x$ \\
\hline 8 & alpha-pinene & 9,59 & 930 & 0,011 & Terpenes & $\mathbf{x}$ & $\mathbf{x}$ & $\mathbf{x}$ \\
\hline 9 & benzaldehyde & 10,53 & 956 & 0,016 & Aldehydes & $\mathbf{x}$ & $\mathbf{x}$ & $\mathbf{x}$ \\
\hline 10 & unknown compound 2 & 10,63 & 959 & 0,017 & - & $\mathbf{x}$ & $x$ & $\mathbf{x}$ \\
\hline 11 & dimethyl trisulfide & 10,86 & 964 & 0,027 & Sulfides & $\mathbf{x}$ & $x$ & $\mathbf{x}$ \\
\hline 12 & C10-decane & 12,19 & 998 & 0,000 & Alkenes & $\mathbf{x}$ & $x$ & $x$ \\
\hline 13 & unknown alkene & 12,41 & 1003 & 0,018 & Alkenes & $\mathbf{x}$ & $\mathbf{x}$ & $\mathbf{x}$ \\
\hline 14 & unknown compound 3 & 13,87 & 1040 & 0,013 & - & $\mathbf{x}$ & $\mathbf{x}$ & $\mathbf{x}$ \\
\hline 15 & S-Methyl methanethiosulfonate & 14,65 & 1059 & 0,010 & Sulfides & $\mathbf{x}$ & & \\
\hline 16 & 1,2,4-Trithiolane & 15,71 & 1082 & 0,012 & Sulfides & $x$ & & $\mathbf{x}$ \\
\hline 17 & unknown compound 4 & 15,89 & 1087 & 0,000 & - & $\mathbf{x}$ & $\mathbf{x}$ & \\
\hline 18 & C11-alkene & 16,47 & 1102 & 0,000 & Alkenes & $\mathbf{x}$ & $\mathbf{x}$ & $\mathbf{x}$ \\
\hline 19 & naphthalene & 19,43 & 1178 & 0,000 & Benzenoids & $\mathbf{x}$ & $\mathbf{x}$ & $\mathbf{x}$ \\
\hline 20 & 2,5-bis(1-methylethyl)-pyrazine & 19,73 & 1186 & 0,001 & Pyrazines & $x$ & & $\mathbf{x}$ \\
\hline 21 & branched alkene & 23,19 & 1284 & 0,008 & Alkenes & $x$ & $\mathbf{x}$ & $\mathbf{x}$ \\
\hline 22 & unknown compound 5 & 30,81 & 1471 & 0,008 & - & $\mathbf{x}$ & $\mathrm{x}$ & $\mathbf{x}$ \\
\hline Num & ber of detected compound & & & & & 22 & 17 & 20 \\
\hline
\end{tabular}

Abbreviations:

\# = Compound number, Burk = Burkholderia sp., AD24, Paen = Paenibacillus sp. AD87, MIX Burk+Paen = Burkholderia sp., AD24 + Paenibacillus sp. AD87. $\mathrm{RT}^{*}=$ Retention time, the RT value stated is the average of three replicates. ELRI $^{* *}=$ Experimental linear retention index value, the RI value stated is the average of three replicates.

p-value ${ }^{* * *}=$ statistical significance (peak area and peak intensity). 


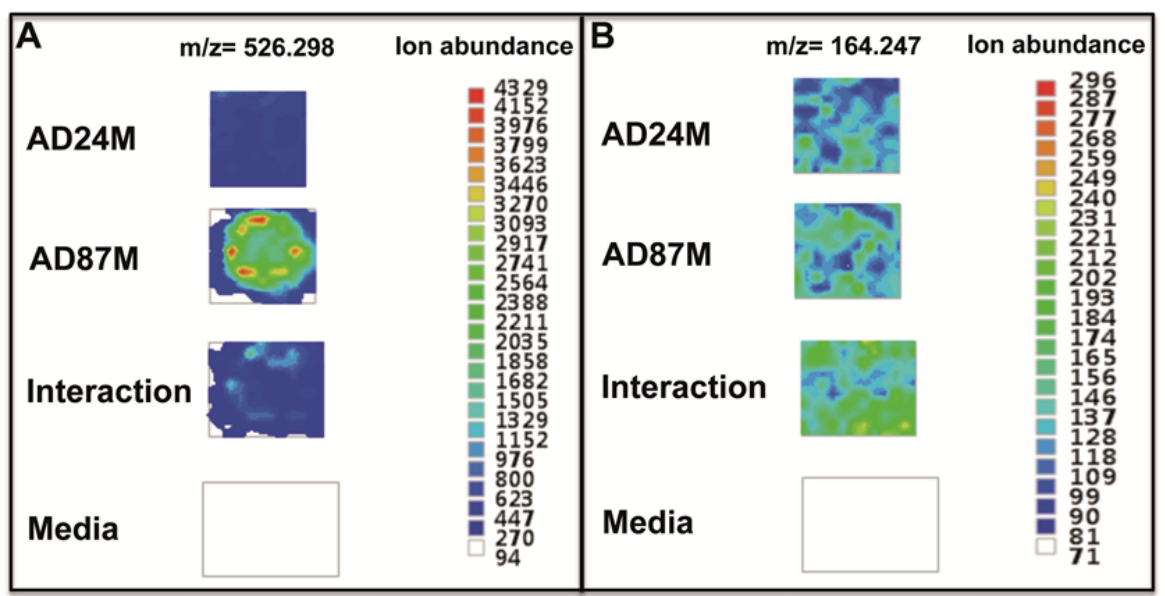

Figure 4.4: Results of the LAESI-MS imaging (A) heat map targeting the

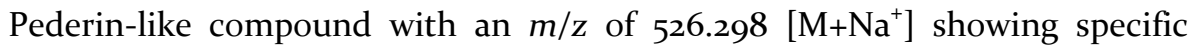
accumulation of ions related to this compound in monocultures of Paenibacillus sp. AD87 (AD87M) and the interaction of Paenibacillus sp. AD87 and Burkholderia sp. AD24 (B) heat map targeting 2,5-bis(1-methylethyl)pyrazine with an $m / z$ of 164.247 showing specific accumulation of ions related to this compound in interaction samples of Burkholderia sp. AD24 with Paenibacillus sp. AD87 (Interaction). The color associated with the ion map represents the base peak intensity (BPI) of $\left[\mathrm{M}+\mathrm{H}^{+}\right]$masses at a 1-ppm window, scale bar of ion abundance on the right side.

\section{Biological activity of 2,5-bis(1-methylethyl)-pyrazine}

After overnight incubation significant growth inhibition of E. coli WA321, S. aureus $533 \mathrm{R}_{4}$ and $C$. albicans BSMY212 was observed by exposure to $1.84 \mathrm{mg}$ of pure 2,5-bis(1-methylethyl)-pyrazine. The bacterial and the yeast-like model organisms exposed to this pure volatile compound showed significant zones of inhibition (ZOI) around the filter paper compared to the controls (Figure 4.5A). Strong growth inhibition on the two plant pathogenic fungi was also observed by application of $1.8 \mathrm{mg}$ 2,5-bis(1-methylethyl)-pyrazine. The mycelial extension of Rhizoctonia solani AG2.2IIIB and Fusarium culmorum PV exposed to the pure volatile compound was significantly reduced as compared to the controls (Figure 4.5B). 


\section{Exploring bacterial interspecific interactions for discovery of novel antimicrobial compounds}

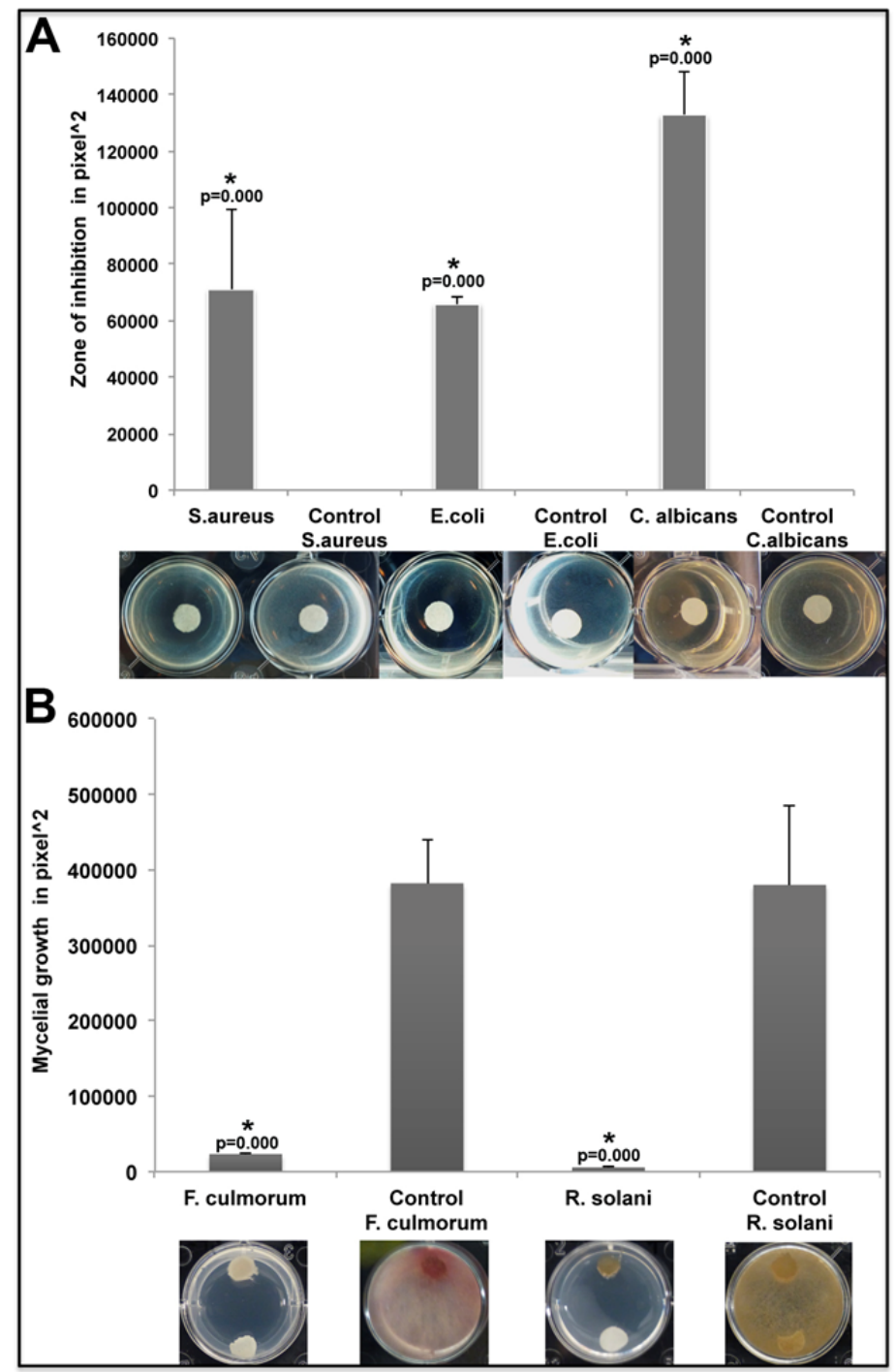

Figure 4.5: Effect of 2,5-bis(1-methylethyl)-pyrazine on growth of target organisms. (A) effect on E.coli WA321, S.aureus $533 \mathrm{R}_{4}$ and C. albicans BSMY212 growth. Bars represent the mean sizes of the Zone of inhibition (ZOI) in pixel $\wedge_{2}$ (B) effect of 2,5 2,5-bis(1-methylethyl)-pyrazine on mycelial extension of Rhizoctonia solani and Fusarium culmorum. Bars represent the mean of mycelial extension in $\operatorname{pixel}^{\wedge}{ }_{2}$. Error bars represent standard deviation (SD). Significant differences between the non exposed control and the treatment are indicated by an asterisk (ONE-WAY ANOVA, post-hoc TUKEY test $\mathrm{p}<0.05)$. 


\section{Antibacterial activity of diffusible secondary metabolites}

Agar diffusion tests performed with secondary metabolite extracts from monocultures and interactions of Burkholderia, Paenibacillus revealed antimicrobial activity against both tested model organisms, namely E.coli $\mathrm{WA}_{321}$ (Figure $4.5 \mathrm{~A}$ ) and S. aureus $533 \mathrm{R}_{4}$ (Figure $\mathbf{S}_{4} .8$ ).

The growth of the model bacteria in the seeded agar around the filter papers supplemented with $5 \mu \mathrm{l}$ of the secondary metabolite extracts was significantly inhibited however without difference in inhibition between extracts obtained from monocultures or interactions (Figure 4.5A, Figure $\mathbf{S}_{4} .8$ ).

\section{Synergistic effect of diffusible and volatile secondary metabolites}

The agar diffusion tests performed with extracts from the interacting bacteria, in combination with $2 \mu \mathrm{l}(=1.84 \mathrm{mg})$ of the pure volatile compound 2,5-bis(1methylethyl)-pyrazine revealed strong synergistic effects between diffusible secondary metabolite extracts and the pure volatile compound against E.coli WA321. The exposure to the secondary metabolite extract in combination with pure 2,5-bis(1-methylethyl)-pyrazine led to significant bigger zones of inhibition (ZOI) around the filter paper compared to the controls (secondary metabolite extracts without added pure volatile compound) (Figure 4.5B). There was no such significant synergistic effect against the Gram-positive model organism $S$. aureus $533 \mathrm{R}_{4}$ by the combination of these compounds (Figure S4.8). 


\section{Exploring bacterial interspecific interactions for discovery of novel antimicrobial compounds}

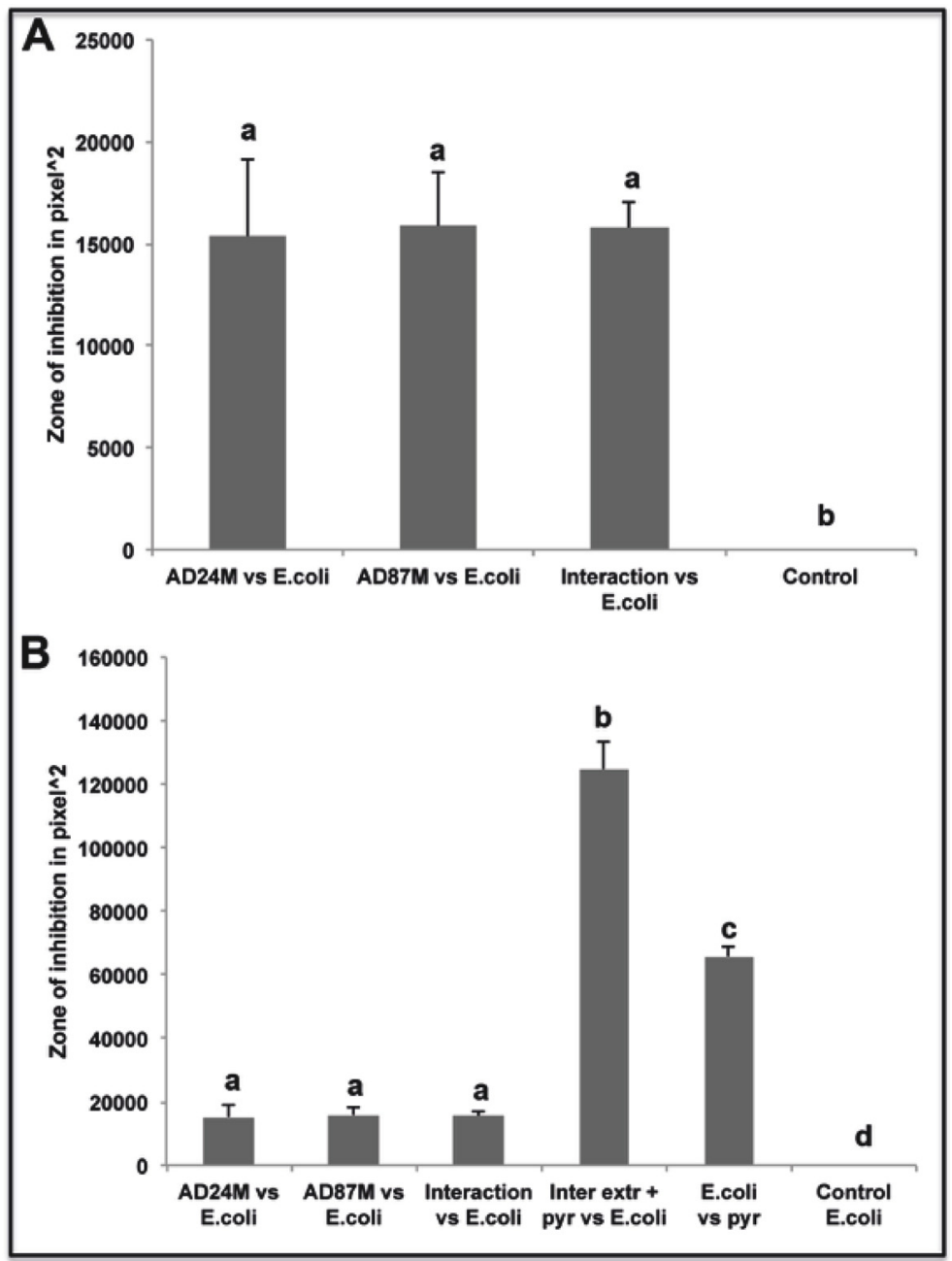

Figure 4.6: Effect of the secondary metabolite extracts of monocultures and interaction of Burkholderia sp. AD24 and Paenibacillus sp. AD87 in combination with 2,5-bis(1-methylethyl)-pyrazine on model organism growth. (A) Effect of the secondary metabolite extracts on E.coli WA321. (B) Effect of the secondary metabolite extracts in combination with $2 \mu \mathrm{l}$ 2,5-bis(1methylethyl)-pyrazine revealing a synergistic effect between the secondary metabolite extracts and the pure pyrazine compound. Bars represent the mean sizes of the Zone of inhibition (ZOI) in pixel` $^{\wedge_{2}}$. Error bars are indicating standard deviation (SD) between the replicates. Significant differences between the treatments are indicated by different letters (ONE-WAY ANOVA, post-hoc TUKEY test $\mathrm{p}<0.05)$. 


\section{Bacterial luciferase reporter assays}

The reporter assays on aqueous extracts from cell material of monocultures and interactions confirmed the elevated inhibition of target strain growth, as measured by the pBPlux reporters for general toxicity. On these samples we deployed also a specific reporter assays for redox cycling compounds, such as phenazines. This reporter gave a clear signal above the detection limit which, upon normalization for the relative toxicity by pBPlux reporters, resulted in a transcriptional signal that reached up to $15 \%$ of the maximum signal induced by the model compound pyocyanin (Figure $\mathbf{S 4 . 9}$ ) in the interaction extract, but not in one of the monoculture extracts.

The volatile compound that exhibited an elevated biosynthesis in the interaction, 2,5-bis(1-methylethyl)-pyrazine was used for bioactivity profiling by bacterial and mammalian reporter assays. Dilutions up to $\sim 3 \cdot 10^{-4} \mathrm{M}$ caused a major reduction in the general toxicity reporter signal (pBPlux-1 in $\mathrm{DH}_{5} \alpha$ and MG1655), while the pBPlux-2 reporter in $\triangle a m p D$ showed a clear decrease from $\sim 1.10^{-3} \mathrm{M}$. The normalized transcriptional signal of the reporter for cell wall synthesis interference (pBLAlux-2 $\triangle a m p D$ ) was induced up to $19.5 \%$ of the maximal transcriptional induction by the model compound penicillin $\mathrm{G}$ at $\sim 3.10^{-4} \mathrm{M}$ (Figure S4.10A, B). The redox cycling (pPHZux-1 $\mathrm{DH}_{5} \alpha$ ) reporter assays did not showed a signal above the detection limit (Figure $\mathbf{S}_{\mathbf{4}} \cdot \mathbf{1 0 C}$ ). The DNA damage reporter pSOSlux-2 MG1655 was induced at nearly maximal induction levels (after normalization), well into the highly toxic concentration range (Figure $\left.\mathbf{S}_{4} \cdot 10 D\right)$.

CALUX $^{\circledR}$ reporter assays for human toxicological assessment of 2,5-bis(1methylethyl)-pyrazine

The mammalian transcriptional reporter assays (CALUX ${ }^{\circledast}$ ) revealed a cytotoxic response at concentration above $10^{-6} \mathrm{M}$, higher concentrations revealed minor induction of the DR-CALUX (induction of the AhR by aromatics such as dioxin-like compounds), ER $\alpha$-CALUX (estrogen-like behavior), Nrf2-CALUX (anti-oxidant response) and p53-CALUX (genotoxicity) (Figure S11A-F). These transcriptional activations occurred at elevated concentrations and can be the cause of the stochastic transcriptional behavior during cytotoxicity). The p53 induction suggests genotoxic mode of action and is confirmed by the pSOSlux-2 (MG1655) data from the bacterial reporter assays. 


\section{Exploring bacterial interspecific interactions for discovery of novel antimicrobial compounds}

\section{Discussion}

Phenotypic changes occurring during microbial interspecific interactions are receiving increased attention as they are the basis for explaining functioning of microbes in complex communities (Seyedsayamdost et al., 2012; Traxler et al., 2013). For example, interspecific interactions between soil bacteria were shown to have a major impact on production of antimicrobial compounds, with both induction and suppression of antimicrobial activity (Tyc et al., 2014). Yet, detailed information on the mechanisms of phenotypic changes during interspecific interactions is still scarce. Here we studied the interspecific interaction between a Gram-positive (Paenibacillus) and a Gram-negative bacterium (Burkholderia), in order to explore how this interspecific interaction will affect bacterial cell numbers, gene expression and the production of antimicrobial secondary metabolites.

The results of the present study revealed that the interaction between both bacteria had a negative effect on Burkholderia cell numbers whereas cell numbers of Paenibacillus appeared not to be affected. Hence it seems that Paenibacillus is a better competitor than Burkholderia under the conditions tested. Similar observations were previously reported for the interspecific interaction between Gram-negative and Gram-positive bacteria (Garbeva et al., 2011b;Tyc et al., 2015). Besides significant reduction of Burkholderia cell counts, we observed also up- and down- regulation of ribosomal proteins. Ribosomal proteins may have various functions apart from protein synthesis. The observed differential expression of ribosomal proteins can point at a general stress response (Ishige et al., 2003;Silberbach and Burkovski, 2006;Picard et al., 2013). Furthermore, ribosomal proteins may be important for antimicrobial activity as reported by de Carvalho (de Carvalho et al., 2010).

Up-regulation of several genes related to signal transduction, secondary metabolite production and to cell motility was observed for Burkholderia during the interaction with Paenibacillus. The elevated expression of gene bAD24_IIo8070 YiaD which is associated with the flagellar biogenesis and the cellular motility apparatus (Hu et al., 2009) indicates that motility can be an important feature during bacterial interspecific interactions. This observation could imply that Burkholderia is trying to move away from Paenibacillus micro colonies. Elevated expression of genes related to cell motility during interspecific bacterial interactions was already reported for Pseudomonas fluorescens Pfo-1 (Garbeva et al., 2011b;Garbeva et al., 2014a). Interestingly the highest fold change in gene expression in Burkholderia was found for the gene 130 
bAD24_po1665 which is related to type IV secretion system. This secretion system plays an important role for the virulence of Burkholderia spp. (Zhang et al., 2009). The gene encoding for this secretion system was found on the mobile genetic element of Burkholderia which is in line with previous reports on type IV secretion systems in Burkholderia spp. (Engledow et al., 2004).

For Paenibacillus, genes encoding antibiotic resistance were upregulated. In particular gene gpAD87_28110 encoding the Vancomycin B-type resistance gene VanW was 9.36 fold up regulated, suggesting protection against antimicrobial compounds produced by Burkholderia. So far, the exact function of gene VanW is yet not completely understood (Evers and Courvalin, 1996;McGregor and Young, 200o). Recently, Letoffe and co-workers (Letoffe et al., 2014) reported the increase of antibiotic resistance due to possible synergistic effects between volatile compounds. Other studies also indicate that VOCs can act as modulator of antibiotic resistance (Kova et al., 2015). Thus it is possible that here, the produced volatile compounds induce increased expression of genes related to antibiotic resistance in Paenibacillus.

Several of the differentially expressed genes in both the Burkholderia and Paenibacillus strains are hypothetical proteins with unknown functions e.g. gpAD87_2500o, gpAD87_1318o, bAD24_I11109o, bAD24_po1610. Despite the advantages made in the field of genome sequencing and genome annotation a vast percentage of bacterial genome sequences remains with unknown function (Galperin and Koonin, 2004;Song et al., 2015). Independent studies also indicated that such "unknown genes" are differentially expressed during interspecific interactions between bacteria (Garbeva et al., 2011b;Garbeva et al., 2014a). It will be important in further studies to determine the function and the products of such unknown genes that are apparently prominent during bacterial interactions.

The metabolomic analysis revealed that the interspecific interaction between Burkholderia and Paenibacillus increased the production of specific antimicrobial compounds such as 2,5-bis(1-methylethyl)-pyrazine and an unknown Pederin-like compound. These two compounds were detected in higher concentrations during interspecific interaction by using three independent approaches namely Orbitrap-XL-MS analysis, GC/MS-Q-TOF analysis and ambient imaging mass spectrometry (LAESI- MS) from living bacterial colonies. The performed bioassays with the volatile compound 2,5bis(1-methylethyl)-pyrazine revealed significant antibacterial and antifungal 


\section{Exploring bacterial interspecific interactions for discovery of novel antimicrobial compounds}

activity against the used microbial strains. Previous studies have already shown that pyrazine compounds can exhibit antimicrobial activities (Beck et al., 2003;Kucerova-Chlupacova et al., 2015). The detected pyrazine is most probably produced by the Burkholderia isolate, as small amounts of this compound were detected in the monoculture of these bacteria but not in monocultures of Paenibacillus. The bacterial production of the volatile 2,5bis(1-methylethyl)-pyrazine was, so far, only reported for few bacteria (Beck et al., 2003;Dickschat et al., 2005; Rajini et al., 2011).

The bioreporter assays performed with this compound revealed that the antimicrobial activity of 2,5-bis(1-methylethyl)-pyrazine is exerted by generating stress at the level of cell wall integrity, which is in line with results of the RNA-sequencing analysis were we observed higher expression of genes related to cell-wall synthesis in both Burkholderia and Paenibacillus. The toxicity of 2,5-bis(1-methylethyl)-pyrazine occurs below the toxic range observed in direct exposure of the CALUX ${ }^{\circledR}$ reporter panel.

Increased antimicrobial effects were seen when the extracts of the interaction and the pure volatile compound 2,5-bis(1-methylethyl)-pyrazine were combined. This might be due to synergetic effects between non-volatile and volatile compounds enhancing the overall antimicrobial activity (Schmidt et al., 2015). Such synergistic effects between volatile and non-volatile compounds were previously observed for hydrophilic antibiotics like e.g. betalactam antibiotics, which showed only negligible antimicrobial effects on Gram-negative bacteria if applied as soluble compound alone (Hemaiswarya and Doble, 2010). However, if these antibiotics were applied together with the volatile organic compound eugenol, increased antimicrobial activity towards Gram-negative and Gram- positive bacteria was observed (Hemaiswarya and Doble, 2010). In addition, combinations of soluble compounds non-volatile and volatile organic compounds have shown to inhibit the growth of multiresistant E. coli and multi-resistant $S$. aureus isolates (Gallucci et al., 2009). Another compound produced in higher concentration during the interspecific interaction of Burkholderia and Paenibacillus was a soluble compound with an $\mathrm{m} / \mathrm{z}$ of $504.316\left[\mathrm{M}+\mathrm{H}^{+}\right]$. So far, we were able to identify this compound as a Pederin like compound (exact mass difference $<0.05 \mathrm{ppm}$ ), however not all gene clusters related to Pederin production were detected in the genome of Paenibacillus. Pederin is a toxic amid with anti-tumoral and mitosis inhibiting properties found in endo-symbiotic Pseudomonads of Paederus beetles (Frank and Kanamitsu, 1987;Piel, 2002; 2009). Bioinformatics analysis revealed that 132 
only three gene clusters (pedB, pedC and pedF) related to the known Pederin synthesis in Pseudomonas fluorescens and Bacillus subtilis with similarities of $37 \%$ to pedB and $29 \%$ to pedC and pedF were present in the genome of Paenibacillus. Additional bioinformatics analysis targeting KS (keto-synthase) domains revealed that only two KS domains are present in the genome of Paenibacillus, however nine KS domains would be needed for the full synthesis of Pederin (Piel, 2002; 2009). This suggests that the detected compound is most probably a novel compound from the same group/chemical class as Pederin. Soil is a very heterogeneous and complex environment consisting of aggregated particles harboring a high density and diversity of bacteria. In this environment bacteria can encounter several different competitors at the same time (Hibbing et al., 2010;Cornforth and Foster, 2013). Thus, production of secondary metabolites with antimicrobial properties such as the volatile 2,5-bis(1-methylethyl)-pyrazine during interspecific interactions may offer advantages during interspecific interactions for the producing strain by inhibition of surrounding competitors.

Our present study on interspecific bacterial interactions has been performed on nutrient rich agar media, these growth conditions are different from the nutritional conditions in natural soils (Torsvik et al., 199ob;Demoling et al., 2007). However, in a previous study a synthetic microbial community consisting out of five bacterial species, including Burkholderia sp. AD24 and Paenibacillus sp. AD87 the volatile compound 2,5-bis(1-methylethyl)-pyrazine was also found to be produced in a soil-microcosm system (Schulz-Bohm et al., 2015). Thus, it is likely that this compound will be also produced during interspecific interactions of Burkholderia in natural systems such as soil. This observation makes this bacterium a promising candidate for pro-biotic treatments in soil. Based on all results, we cannot exclude the application of 2,5-bis(1-methylethyl)-pyrazine for applications like fumigation in logistics, food, or for plant protection against plant-pathogenic fungi and bacteria in agricultural production systems (Audrain et al., 2015).

In conclusion, the present study revealed that interspecific bacterial interactions affected fitness, gene expression and secondary metabolite profiles (volatile and non-volatile compounds). A graphical summary of the transcriptome and metabolome analysis and the mechanisms involved during the interspecific interaction between Paenibacillus sp. and Burkholderia is presented in Figure 4.7. 


\section{Exploring bacterial interspecific interactions for discovery of novel antimicrobial compounds}

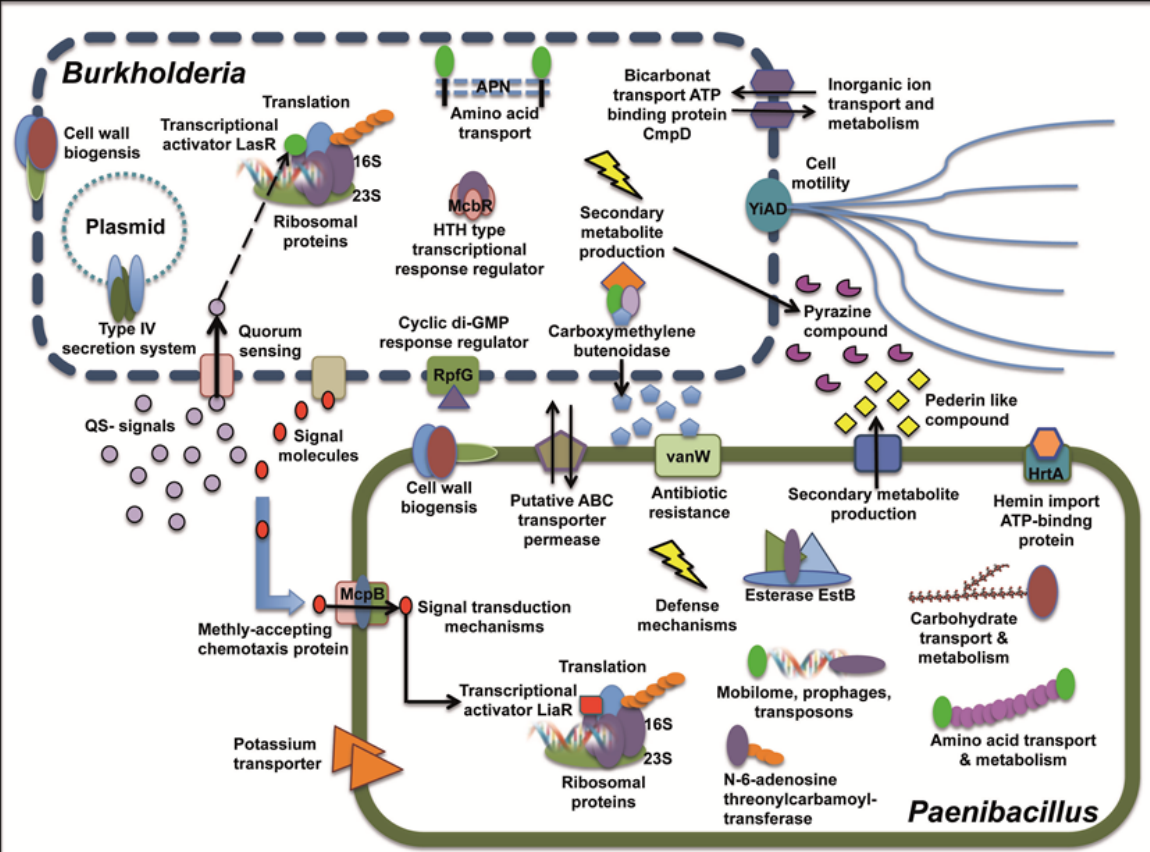

Figure 4.7: Schematic overview of the most important changes in gene expression and metabolite production in Burkholderia sp. AD24 (top) and Paenibacillus sp. AD87 (bottom) during interspecific interaction. This overview additionally includes the two secondary metabolites 2,5-bis(1methylethyl)-pyrazine and the Pederin like compound which were detected in higher concentrations during interspecific interaction using mass spectrometric technologies.

The knowledge obtained here can be beneficial for the construction of synthetic microbial communities that consists of a minimal set of microorganisms needed to fulfil specific ecosystem services like e.g. disease suppression in agricultural systems (Shong et al., 2012;De Roy et al., 2013;Grosskopf and Soyer, 2014).

The observed triggering of a volatile pyrazine compound production during interspecific interaction indicates that screening of interspecific interactions may lead to the discovery of novel volatile compounds with antimicrobial activities. 


\section{Acknowledgments}

This work was supported by the BE-Basic Foundation (http://www.be-basic.org/). PG is financed by The Netherlands Organization for Scientific Research (NWO) VIDI personal grant 864.11.015. The authors want to thank Kristin Schulz-Bohm for her useful advices on the qPCR assays, Prof. Dr. Harald Gross and Judith Bauer for their advices on the Pederin like compound identification. This is publication XXXX of the NIOO-KNAW. 


\section{Exploring bacterial interspecific interactions for discovery of novel antimicrobial compounds}

\section{Supplementary material}

Table S4.1: De-Novo genome assembly statistics of the two sequenced bacterial isolates.

\begin{tabular}{lrr}
\hline & Burkholderia sp. AD24 & Paenibacillus sp. AD87 \\
\cline { 2 - 3 } Number of PacBio subreads & 57683 & 116628 \\
N50 subreads & 6956 & 7032 \\
PacBio coverage & 31 & 36,65 \\
Illumina coverage & 79 & 200 \\
number of Illumina read pairs & 3515383 & 5018466 \\
contigs HGAP 3 & 10 & 36 \\
contigs scaffolding & 4 & 7 \\
Final number of scaffolds & 4 & 30 \\
Final number of contigs & 3 & 37 \\
Number of gaps & 17 & 7 \\
N50 & 4476846 & 5194573 \\
\hline Total genome size & $\mathbf{8 2 4 3 4 4 0}$ & $\mathbf{7 0 8 6 7 1 3}$
\end{tabular}

Table S4.2: Primers used for quantitative real-time PCRs.

\begin{tabular}{llll}
\hline \multicolumn{1}{c}{ Primer code } & \multicolumn{1}{c}{ Sequence $\left(\mathbf{5}^{\prime} \mathbf{-}\right.$-3') of primers } & \multicolumn{1}{c}{ target } & reference \\
\hline gpAD87_304F & GTACTTCCCGCACCTGACAT & dimodular nonribosomal peptide synthase & this study \\
gpAD87_304R & TGGCGAGAACTCCACTTCT & dimodular nonribosomal peptide synthase & this study \\
bAD24_10391_IG_F & GTATTGGCCGTATCCGTCAG & snoaL-like polyketide cyclase family protein & this study \\
bAD24_10391_IG_R & AGCCACTCTTCGACGATCAC & snoaL-like polyketide cyclase family protein & this study \\
RecA_bAD24_1_F & GGTGAGGCAATCGAAGACAT & DNA recombination and repair protein & this study \\
RecA_bAD24_1_R & AGCTTGCTTGCGTACTGGAT & DNA recombination and repair protein & this study \\
RecA_gpAD87_3_F & CTTGCCTAAAGGCCGTATTG & DNA recombination and repair protein & this study \\
RecA_gpAD87_3_R & GACAATGTCCACAGCACCAC & DNA recombination and repair protein & this study \\
BacF & GGGAAACCGGGGCTAATACCGGAT & 16S rDNA specific for Bacilli & Garbeva et al. (2003) \\
Eub338F & ACTCCTACGGGAGGCAGCAG & 16S rDNA & Fierer et al. (2005) \\
Eub518R & ATTACCGCGGCTGCTGG & 16S rDNA & Fierer et al. (2005)
\end{tabular}


Table S4.3: Primers used in bioreporter construction.

\begin{tabular}{ll}
\hline \multicolumn{1}{c}{ Primer code } & \multicolumn{1}{c}{ Sequence $\left(\mathbf{5}^{\prime} \mathbf{~} \mathbf{3} \mathbf{3}^{\prime}\right)$ of primers } \\
\hline Primer 1 & AAAAAGGATCCGGCGGGTACTCCGGG \\
Primer 2 & AAAAACTCGAGCCGCTGCCTAGCCGTCG \\
Primer 3 & AAAAAGAATCCGATTGGTTGGTTCCCTGGGTTCTGG \\
Primer 4 & AAAAACTCGAGCTAGCCGGCCACCGCTTCC \\
Primer 5 & AAAAATTAATTAAATCAGAACGCAGAAGCGG \\
Primer 6 & AAAAATTAATTAACCTATATCGCCGACATCACC \\
Primer 7 & TCGTCTTCACCTCGAGTGCCAGTCGGCGCTCTTC \\
Primer 8 & CGCAACTAGAGGATCCAAACACCTCTTTGAC \\
Primer 9 & TCGAAATACTTGACATATCACTGTGATTCACATATAATATGCG \\
Primer 10 & GATCCGCATATTATATGTGAATCACAGTGATATGTCAAGTATT
\end{tabular}

Table S4.4: Bacterial strains and plasmids used for bioreporter construction and the performed bioreporter assays on aqueous and pure volatile extracts.

\begin{tabular}{|c|c|c|}
\hline & Description & Reference \\
\hline \multicolumn{3}{|c|}{ Strain } \\
\hline E. coli DH5 $\alpha$ & $\begin{array}{l}\text { F-, } \Delta(\operatorname{argF}-\mathrm{lac}) 169, \varphi 80 \mathrm{dlacZ58}(\mathrm{M} 15), \\
\Delta \text { phoA8, glnX44(AS), } \lambda-, \text { deoR481, rfbC1?, } \\
\text { gyrA96(NalR), recA1, endA1, thiE1, hsdR17 }\end{array}$ & \\
\hline E. coli MG1655 & $\mathrm{F}-, \lambda-, \mathrm{rph}-1$ & \\
\hline E. coli $\Delta a m p D$ & $\begin{array}{l}\text { F-, } \Delta(\operatorname{araD}-a r a B) 567, \Delta \text { ampD728::kan, } \\
\Delta \text { lacZ4787(::rrnB-3), } \lambda-, \text { rph-1, } \Delta(\text { rhaD- } \\
\text { rhaB)568, hsdR514 }\end{array}$ & Baba et al (2006) \\
\hline$P$. aeruginosa PA14 & \multirow{2}{*}{ Wild type, as PCR template } & \\
\hline P. protegens Pf-5 & & USDA-ARS \\
\hline \multicolumn{3}{|c|}{ Plasmid } \\
\hline pCS26Pac & $\mathrm{Km}^{\mathrm{r}}, \mathrm{pSC} 101, \mathrm{lu} \times \mathrm{CDABE}$ & Bjarnason et al (2003) \\
\hline pBAD33 & $\mathrm{Cm}^{\mathrm{r}}, \mathrm{pACYC} 184$ & Guzman et al (1995) \\
\hline pJAMA8-cda & $\begin{array}{l}\mathrm{Ap}^{\mathrm{r}} \text {, ColE1, IuxAB promoter-probe vector } \\
\text { with the cda (colicin D) promoter. }\end{array}$ & Tecon et al (2010) \\
\hline pBPlux-1 & & This study \\
\hline pBPlux-2 & & This study \\
\hline pPHZlux-1 & & This study \\
\hline pSOSlux-2 & & This study \\
\hline pBLAlux-1 & & This study \\
\hline pBLAlux-2 & & This study \\
\hline
\end{tabular}




\section{Exploring bacterial interspecific interactions for discovery of novel antimicrobial compounds}

Table S4.5: Outcome of the qRT-PCR analysis and comparison to the outcome of the RNA-seq analysis. The fold change values for the qRT-PCR are analysis are calculated with the $\Delta \Delta$-CT method.

\begin{tabular}{|c|c|c|c|c|c|}
\hline Gene & function & time point & $\begin{array}{c}\text { fold change qPCR } \\
\qquad \Delta-\mathrm{CT}\end{array}$ & SD qPCR & $\begin{array}{l}\text { fold change RNA- } \\
\text { seq data }\end{array}$ \\
\hline bAD24_10391 & snoaL-like polyketide cyclase family protein & $\mathrm{t} 48$ & 1,30 & \pm 0.73 & 1,61 \\
\hline bAD24_10391 & snoaL-like polyketide cyclase family protein & $\mathrm{t} 72$ & 1,20 & \pm 0.50 & 1,21 \\
\hline gpAD87_304 & $\begin{array}{l}\text { dimodular nonribosomal peptide synthase } \\
29 \% \text { similiarity with pedF }\end{array}$ & t48 & 0,75 & \pm 0.33 & 0,51 \\
\hline gpAD87_304 & $\begin{array}{l}\text { dimodular nonribosomal peptide synthase } \\
29 \% \text { similiarity with pedF }\end{array}$ & $\mathrm{t} 72$ & 1,35 & \pm 0.81 & 1,34 \\
\hline
\end{tabular}

Normalized Conc.

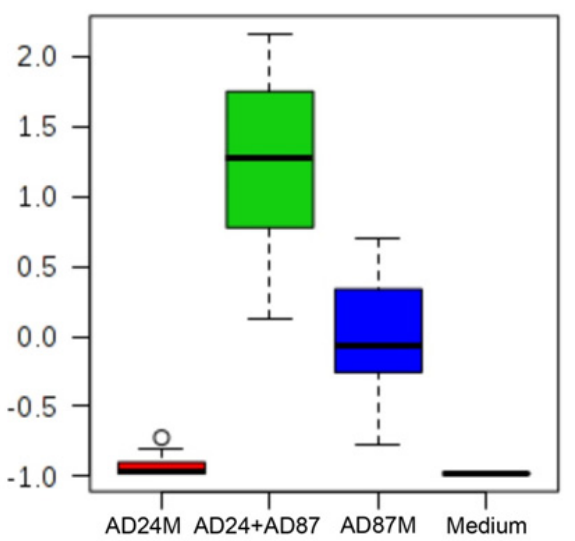

Figure S4.6: Normalized concentration of the unknown Pederin like compound with a mass of $504.316\left(\mathrm{M}+\mathrm{H}^{+}\right)$. The compound was detected in a higher concentration during interaction of Burkholderia sp. AD24 with Paenibacillus sp. AD87. The compound was not detected in samples of Burkholderia sp. AD24 monoculture and in the medium control (extracts of $1 / 10^{\text {th }}$ TSBA). 


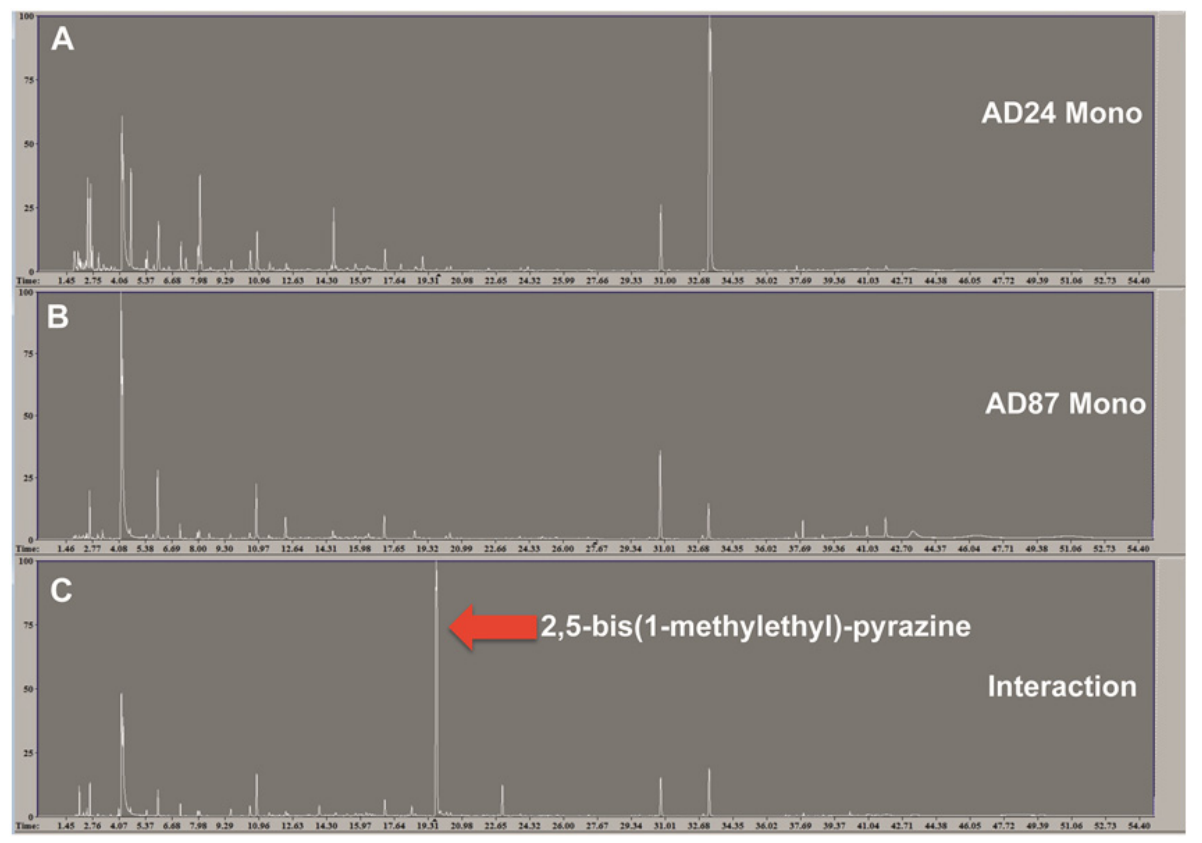

Figure S4.7: Representation of GC/MS chromatograms of (A) Burkholderia sp. AD24 monoculture (top) (B) Paenibacillus sp. AD87 monoculture (middle) and $(\mathbf{C})$ interaction of both bacteria (bottom). The compound 2,5-bis(1methylethyl)-pyrazine (RT $19.7 \mathrm{~m} / \mathrm{z}=164.247$ ) was detected in a higher concentration in samples of the pairwise combination of Burkholderia sp. AD24 with Paenibacillus sp. AD87. 


\section{Exploring bacterial interspecific interactions for discovery of novel antimicrobial compounds}

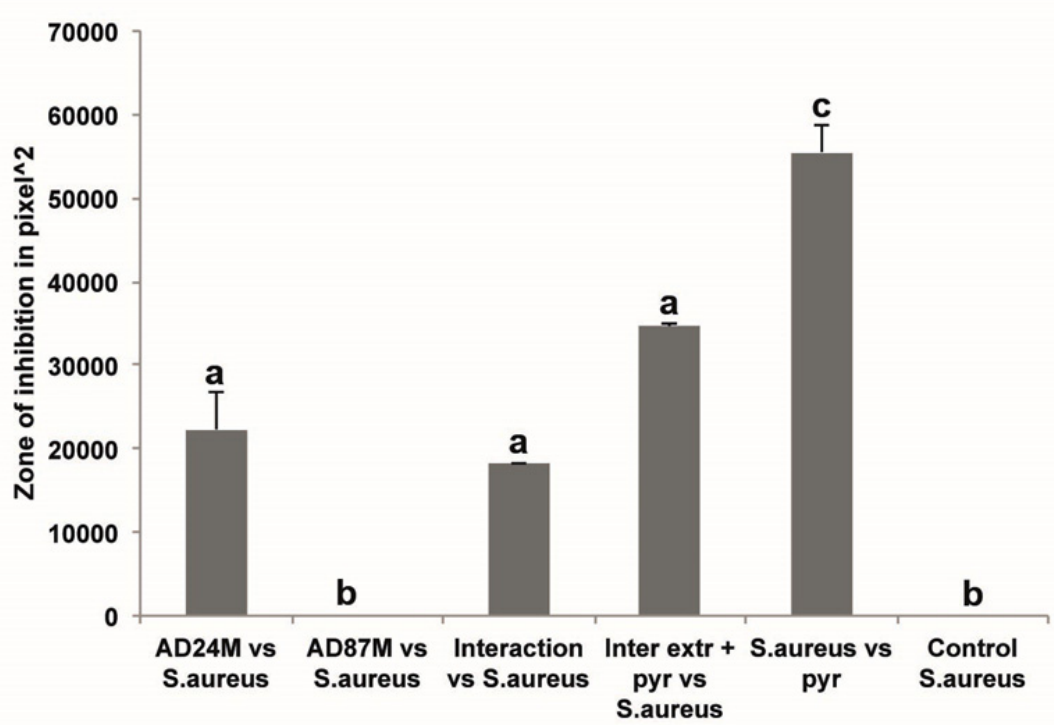

Figure S4.8: Effect of the secondary metabolite extracts and the interaction extract in combination with 2,5-bis(1-methylethyl)-pyrazine on growth of S.aureus $533 \mathrm{R}_{4}$. Bars represent the mean sizes of the Zone of inhibition (ZOI) in $\operatorname{pixel}^{\wedge}{ }^{\wedge}$. Error bars are indicating standard deviation (SD) between the replicates. Significant differences between the control and the treatments are indicated by different letters (ONE-WAY ANOVA, post-hoc TUKEY test $\mathrm{p}<0.05)$. 
A

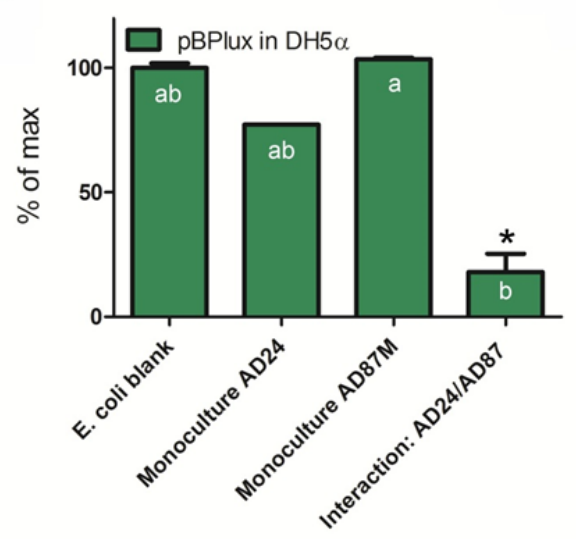

B

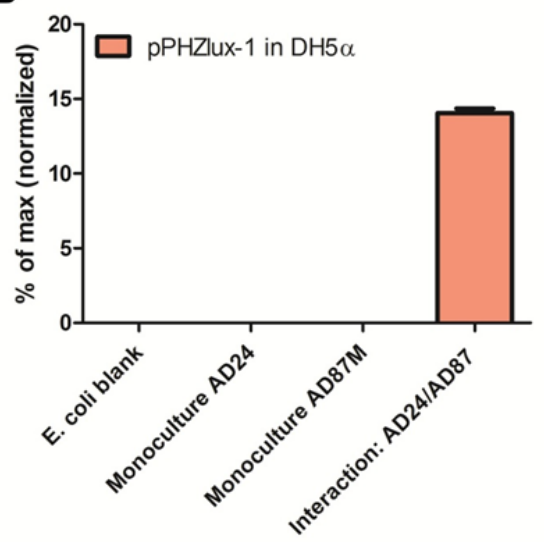

Figure S4.9: Aqueous extracts were used for exposure of the bacterial reporters for toxicity (pBPlux), measured as a reduction luminescence, and redox cycling by induction of pPHZlux-1. The resulting signals were represented as \% of the maximum in the blank sample (A) or the reference compound pyocyanine (B) respectively. The interaction showed a significant toxicity relative to the blank and the monocultures. The extracts from the interaction revealed a redox cycling activity, as measured by the pPHZlux-1 reporter. Above detection limit measurements of the latter assay were corrected for toxicity. Significance levels ${ }^{*} \mathrm{p}<0.05$ after Kruskal-Wallis test (Dunn post-hoc multiple comparison) in GraphPad Prism. 


\section{Exploring bacterial interspecific interactions for discovery of novel antimicrobial compounds}

A

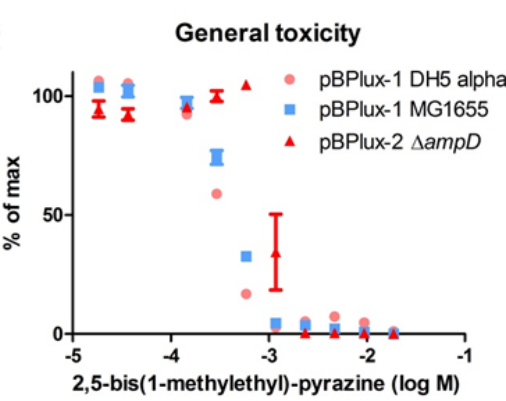

C pPHZlux-1 DH5 $\alpha$ (redox cycling)

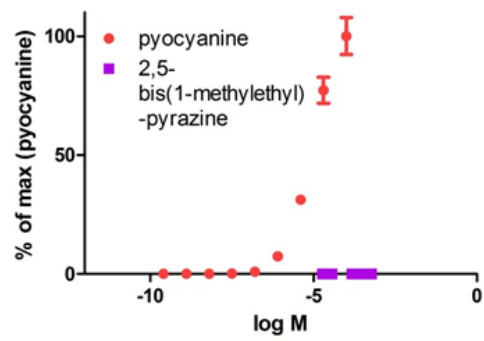

B pBLALux-2 $\triangle a m p D$ (cell wall integrity)

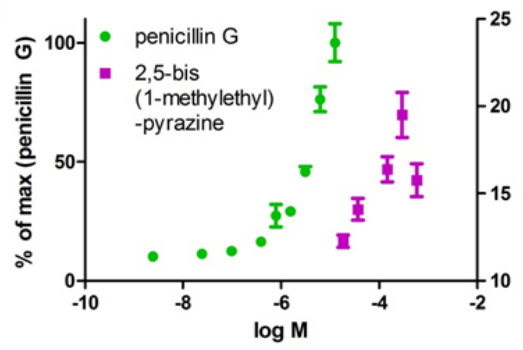

D pSOSlux-2 MG1655 (DNA damage)

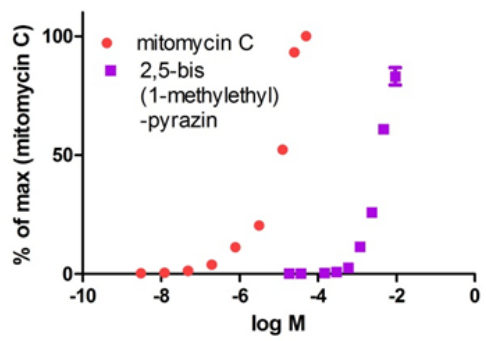

Figure S4.10: Overview of the dose response curves of the bacterial reporter assays upon 2,5-bis(1-methylethyl)-pyrazine (A) The synthesized pyrazine proved to the E. coli host as revealed by the three control reporter strains. (B) The reporter that detects interference with cell wall synthesis and integrity, pBLAlux-2 $(\Delta \mathrm{ampD})$ was induced after normalization to toxicity up to $19.5 \%$ of the maximum response of the model compound penicillin $\mathrm{G}\left(\right.$ at $\left.\sim 3.10^{-4} \mathrm{M}\right)$. b: pPHZlux-1 DH5 $\alpha$. (C) The reporter for redox cycling compounds was not induced at all by this compound. (D) pSOSlux-2 MG1655 exhibited a normalized response of up $83 \%$ of the reference compound at elevated concentrations, within the toxic range. 
A

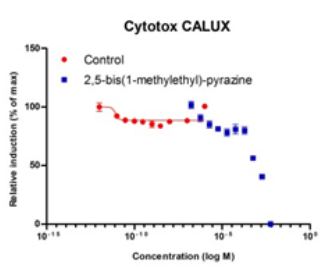

D

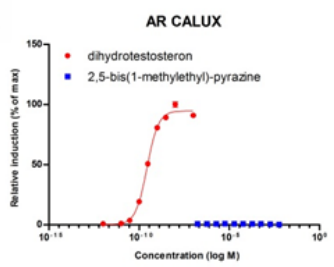

B

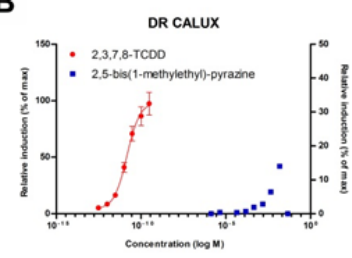

C
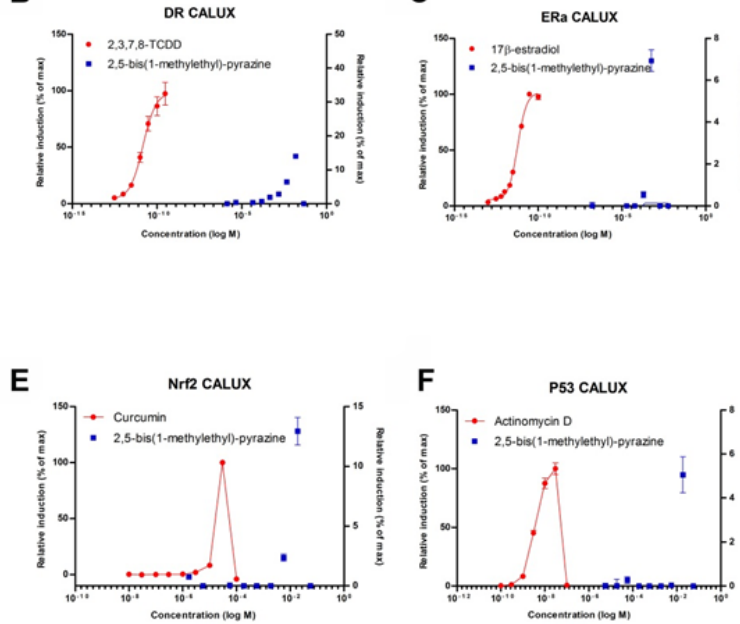

F P53 CaluX

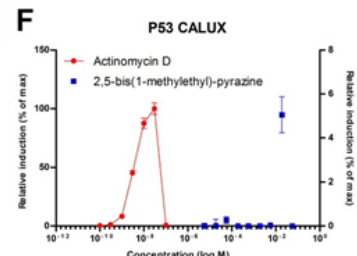

Figure S4.11: Overview of the selection of CALUX ${ }^{\circledR}$ mammalian reported assays exposed to the 2,5-bis(1-methylethyl)-pyrazine as compared to the response of the model compounds. The dose responses are represented as concentrations in the wells. Despite the cytotoxicity above $1 \mathrm{oE}^{-4} \mathrm{M}$ (A), adverse toxicological endpoints, such as dioxin-like (B), estrogen-like (C), Nrf2-like, (D) Androgenic activity was not observed with this compound. (E) and genotoxic activity (F). Responses are observed at elevated levels up to $10^{-2} \mathrm{M}$. When present, the right axis represent the relative response of 2,5-bis(1-methylethyl)-pyrazine. 
Exploring bacterial interspecific interactions for discovery of novel antimicrobial compounds 


\section{Chapter 5}

\section{The effect of phylogenetically different bacteria on the fitness of Pseudomonas fluorescens in sand microcosms}

Olaf Tyc, Alexandra B. Wolf and Paolina Garbeva

This chapter has been published as:

Tyc, O. Wolf, A. Garbeva, P. (2015). The effect of phylogenetically different bacteria on the fitness of Pseudomonas fluorescens in sand microcosms. PLoS One, 10 doi:10.1371/journal.pone.0119838 


\title{
The effect of phylogenetically different bacteria on the fitness of Pseudomonas fluorescens in sand microcosms
}

\begin{abstract}
In most environments many microorganisms live in close vicinity and can interact in various ways. Recent studies suggest that bacteria are able to sense and respond to the presence of neighboring bacteria in the environment and alter their response accordingly. This ability might be an important strategy in complex habitats such as soils, with great implications for shaping the microbial community structure.
\end{abstract}

Here, we used a sand microcosm approach to investigate how Pseudomonas fluorescens Pfo-1 responds to the presence of monocultures or mixtures of two phylogenetically different bacteria, a Gram-negative (Pedobacter sp. V48) and a Gram-positive (Bacillus sp. V102) under two nutrient conditions. Results revealed that under both nutrient poor and nutrient rich conditions confrontation with the Gram-positive Bacillus sp. V102 strain led to significant lower cell numbers of Pseudomonas fluorescens Pfo-1, whereas confrontation with the Gram-negative Pedobacter sp. $\mathrm{V}_{4} 8$ strain did not affect the growth of Pseudomonas fluorescens Pfo-1. However, when Pseudomonas fluorescens Pfo1 was confronted with the mixture of both strains, no significant effect on the growth of Pseudomonas fluorescens Pfo-1 was observed. Quantitative real-time PCR data showed up-regulation of genes involved in the production of a broad-spectrum antibiotic in Pseudomonas fluorescens Pfo-1 when confronted with Pedobacter sp. V48, but not in the presence of Bacillus sp. V102.

The results provide evidence that the performance of bacteria in soil depends strongly on the identity of neighboring bacteria and that interspecific interactions are an important factor in determining microbial community structure. 


\section{Introduction}

Culture-independent technologies have given us insight in the tremendous phylogenetic and functional diversity of microbial communities (Gans et al., 2005; Uroz et al., 2010). Recently, the role of interactions between the members of microbial communities and how these shape community composition and dynamics is receiving increasing interest (Hibbing et al., 2010;Foster and Bell, 2012;Cornforth and Foster, 2013;Mitri and Foster, 2013). Both theoretical models and empirical studies are used to explain the coexistence of competing microbial species and consequently microbial community assembly (D'Onofrio et al., 2010;Foster and Bell, 2012).

In soil and in the rhizosphere, many microbial species live in close vicinity and interact with each other in various ways ranging from competition to cooperation (Czaran and Hoekstra, 2009;Foster and Bell, 2012;Allen and Nowak, 2013). Bacteria can recognize cues from their environment to modulate behavior in order to increase their chance of survival.

Using recently developed techniques (NanoDESI and MALDI-TOF imaging mass spectrometry) Traxler and co-authors indicated the importance of interspecific interactions for triggering the production of different secondary metabolites in a single strain (Traxler et al., 2013). Recent studies in our group also indicate that bacteria are respond differently to the presence of different microbial species (de Boer et al., 2007;Garbeva and de Boer, 2009;Garbeva et al., 2011b). Studies on behavior and the transcriptional responses of the soil bacterium Pseudomonas fluorescens Pfo-1 on nutrient-poor agar in confrontation with taxonomically different bacterial species revealed significant differences in the responses of Pseudomonas fluorescens Pfo-1 to different bacteria. In particular, the expression of genes involved in signal transduction and antibiotic production was strongly affected by the identity of the interacting strains (Garbeva et al., 2011b).

So far the response of Pseudomonas fluorescens Pfo-1 to phylogenetically different bacteria has only been studied during one-to-one confrontations on agar media (Garbeva and de Boer, 2009;Garbeva et al., 2011b). However, these conditions are very artificial compared to the situation in the natural soil environment, which is a heterogeneous and complex habitat consisting of aggregated particles with huge bacterial diversity (Torsvik et al., 1990a;Gans et al., 2005;Demoling et al., 2007). It is thus plausible that bacteria can sense more easily the presence of neighbors in their vicinity on an agar plate than in soil. 
Furthermore, in natural environments bacteria are likely to encounter several different competitors at the same time or in sequential events (Hibbing et al., 2010). In the present study, we made a first attempt to study bacterial interactions in soil-like systems.

To this end we investigated the interaction between Pseudomonas fluorescens Pfo-1 with monocultures and mixtures of Pedobacter sp. $\mathrm{V}_{4} 8$ and Bacillus sp. V102 in sand microcosms under two different nutrient conditions. We hypothesized that both nutrient conditions and the identity of the competitor would have an effect on the performance of Pseudomonas fluorescens Pfo-1. 


\section{Materials and methods}

\section{Bacterial strains and growth conditions}

Three different bacterial species, Pseudomonas fluorescens Pfo-1 ( $\gamma-$ Proteobacteria) (Compeau et al., 1988), Pedobacter sp. V48 (Sphingobacteria) and Bacillus sp. V102 (Bacilli) (de Boer et al., 2007) were used in this study (Table 5.1). The strains were pre-cultured from frozen $-8 \mathrm{o}^{\circ} \mathrm{C}$ glycerol stocks on $1 / 10^{\text {th }}$ TSB agar (5.0 $\mathrm{gL}^{-1} \mathrm{NaCl}$ (Merck), $1.0 \mathrm{gL}^{-1} \mathrm{KH}_{2} \mathrm{PO}_{4} ; 3 \mathrm{gL}^{-1}$ Tryptic Soy Broth (OXOID); $20 \mathrm{gL}^{-1}$ Agar (Merck), pH 6.5) (Garbeva and de Boer, 2009) for three days at $20^{\circ} \mathrm{C}$.

Table 5.1: Bacterial strains and used antibiotics / selection method.

\begin{tabular}{|c|c|c|c|}
\hline Bacterial strain & Description & Reference & Selective antibiotic \\
\hline P. fluorescens Pfo-1 & $\begin{array}{l}\text { Wild type, soil isolate, Gram-negative, } \\
\text { Class: Gamma-proteobacteria }\end{array}$ & Compeau et al., (1988) & Ampicillin \\
\hline Pedobacter sp. $\mathrm{V}_{4} 8$ & $\begin{array}{l}\text { Wild type, Gram-negative, Phylum: } \\
\text { Bacteroidetes, Class: Sphingobacteria }\end{array}$ & de Boer et al., (2007) & Kanamycin \\
\hline Bacillus sp. V102 & Wild type, Gram-positive, Class: Bacilli & de Boer et al., (2003) & n/a pasteurization \\
\hline
\end{tabular}

\section{Microcosm setup}

Microcosms were established in $100 \mathrm{~mL}$ glass vials with a plastic screw cap lid (Figure S5.1) containing sterile acid washed sea sand with pore size fractions ranging from 0.075 to $0.425 \mathrm{~mm}$ (Honeywell Specialty Chemicals Seelze $\mathrm{GmbH}$, Germany). The amount of sand was either $25 \mathrm{~g}$ (Microcosms supplemented with $1.5 \mathrm{~mL} 1 / 10^{\text {th }}$ strength Tryptic Soy Broth (nutrient rich media) (5.o gL $\mathrm{gLCl}^{-1} \mathrm{Na}$ (Merck), $1.0 \mathrm{gL}^{-1} \mathrm{KH}_{2} \mathrm{PO}_{4} ; 3 \mathrm{gL}^{-1}$ Tryptic Soy Broth (OXOID)) or $30 \mathrm{~g}$ (Microcosms supplemented with $1.5 \mathrm{~mL}$ nutrient poor media (5.0 gL $\mathrm{gLCl}^{-1} \mathrm{Nerck}$ ), $1.0 \mathrm{gL}^{-1} \mathrm{KH}_{2} \mathrm{PO}_{4}$; $0.1 \mathrm{gL}^{-}\left(\mathrm{NH}_{4}\right)_{2} \mathrm{SO}_{4}$; $0.5 \mathrm{gL}^{-1}$ Tryptic Soy Broth (OXOID)). The sand was weighed directly into the glass vials and afterwards sterilized by autoclaving for 20 minutes. The sterilized microcosms were dried overnight in a $60{ }^{\circ} \mathrm{C}$ oven prior to inoculation. All treatments were performed in triplicates over a time of 6 days. A detailed overview of all treatments and controls is given in Table 5.2. 


\section{The effect of phylogenetically different bacteria on the fitness of Pseudomonas fluorescens in sand microcosms}

Table 5.2: Treatment overview of the microcosm treatments.

\begin{tabular}{|c|c|c|c|}
\hline $\begin{array}{l}\text { Treatment } \\
\text { codec }\end{array}$ & Involved bacterial strains & $\begin{array}{l}\text { Number of tested } \\
\text { interactions }\end{array}$ & Supplied growth media \\
\hline 1 & P. fluorescens Pfo-1, Pedobacter sp. $V_{4} 8$, Bacillus sp. V1o2 & 3 & 1/1oth TSB \\
\hline 2 & P. fluorescens Pfo-1, Pedobacter sp. $\mathrm{V}_{4} 8$ & 2 & 1/1oth TSB \\
\hline 3 & P. fluorescens Pfo-1, Bacillus sp. V102 & 2 & 1/1oth TSB \\
\hline 4 & P. fluorescens Pfo-1, Pedobacter sp. $V_{4} 8$, Bacillus sp. V102 & 3 & Nutrient poor media \\
\hline \multicolumn{4}{|c|}{ Controls } \\
\hline 7 & P. fluorescens Pfo-1 Monoculture & - & 1/1oth TSB \\
\hline 8 & Pedobacter sp. $\mathrm{V}_{4} 8$ Monoculture & - & 1/1oth TSB \\
\hline 12 & Bacillus sp. V1o2 Monoculture & - & Nutrient poor media \\
\hline
\end{tabular}

\section{Microcosm inoculation}

Sand microcosms were inoculated with either each strain as monoculture, pairwise combinations, or with all three strains together (Table 5.2). To inoculate the microcosms a single colony of the respective strain was transferred into $10 \mathrm{ml} \mathrm{of} 1 / 10^{\text {th }}$ TSB and grown overnight at $20{ }^{\circ} \mathrm{C}, 220 \mathrm{rpm}$ to an optical density $\left(\mathrm{OD}_{600}\right)$ of: $\sim 0.700$ (Pseudomonas fluorescens Pfo-1), 0.600 (Pedobacter sp. V48) and $\sim 0.650$ (Bacillus sp. V102). The bacterial strains were diluted in a nutrient rich or nutrient poor inoculation master mix to a density of $\sim 1 * 10^{5} \mathrm{CFU} / \mathrm{mL}$. Each microcosm was inoculated with a volume of $1.5 \mathrm{~mL}$ of the respective inoculum master mix in the middle of the sterilized sand and mixed well.

To verify bacterial cell numbers in the inoculum, dilution plating was done in duplicates on selective agar plates (Pseudomonas fluorescens Pfo-1: $1 / 10^{\text {th }}$ TSBA plates supplemented with $100 \mu \mathrm{g} / \mathrm{mL}$ Ampicillin, Pedobacter sp. $\mathrm{V}_{4} 8: 1 / 10^{\text {th }}$ TSBA plates supplemented with $50 \mu \mathrm{g} / \mathrm{mL}$ Kanamycin, Bacillus sp. Vioz: samples were pasteurized by heat treatment for $10 \mathrm{~min}$. @ $80^{\circ} \mathrm{C}$ ). 


\section{Bacterial enumeration}

The growth of the three bacterial strains in the different treatments was tracked by plate counting of all culturable cells (Pseudomonas fluorescens Pfo1 and Pedobacter sp. V48) or by counting of spores and heat-stable cells (Bacillus sp. V102) (Table 5.1). The enumeration was performed as follows: after one and six days of incubation a sterilized stainless steel spoon was used for sampling by mingling the sand by a full clock- and one counter- clockwise turn. After mixing $1 \mathrm{~g}$ sand was taken from the center of each microcosm and transferred into a $15 \mathrm{~mL}$ Greiner tube. A volume of $10 \mathrm{ml} 10 \mathrm{mM}$ phosphate buffer ( $\mathrm{pH}$ 6.5) was added and the tubes were shaken in a rotary shaker at $350 \mathrm{rpm}$ for 30 minutes at $20^{\circ} \mathrm{C}$. Subsequently, serial dilutions were prepared and plated in triplicates on selective media (antibiotics used are indicated in Table 5.1). For the enumeration of the Bacillus sp. V102, samples were pasteurized by heating the tubes to $80{ }^{\circ} \mathrm{C}$ for $10 \mathrm{~min}$ in a pre-warmed heating block. The plates were incubated for two to four days at $20^{\circ} \mathrm{C}$ and the CFUs of the respective strains were determined.

\section{RNA extraction and quantitative real time PCR}

The expression of gene cluster Pflo1_3463-3466, which is involved in the production of a broad-spectrum antibiotic (Garbeva et al., 2011b) was quantified via quantitative real time PCR. Total RNA was extracted at day 6 from nutrient rich microcosms $\left(1 / 10^{\text {th }}\right.$ TSB) containing Pseudomonas fluorescens Pfo-1 as monoculture or in interaction as follows: the double volume (2 mL) of RNA protect Bacteria Reagent (QIAGEN cat\# 76506) was added to $1 \mathrm{~g}$ sand sample and centrifuged at 10,000 $\mathrm{x} \mathrm{g}$ for $10 \mathrm{~min}$ (Sigma $3 \mathrm{~K}-14$ centrifuge, SIGMA Laborzentrifugen $\mathrm{GmbH}$, Germany). The supernatant was discarded and the pellets were stored at $-80{ }^{\circ} \mathrm{C}$ until further analysis. Total RNA was extracted with the MO-BIO PowerSoil Total RNA Isolation Kit (MOBIO cat\# 12866-25) according to the manufacturer's protocol. The RNA extracts were treated with the TURBO DNA free Kit from AMBION (cat\# 1907) according to the manufacturer's protocol to remove any remaining DNA. The RNA concentration and quality was checked on a NanoDrop Spectrophotometer (Isogen Life Science, IJssestein, The Netherlands). cDNA was synthesized from the extracted RNA with random hexamer primers from Invitrogen (cat\# 48190-011) by using reverse transcriptase of the Fermentas RevertAid Premium First Strand cDNA Synthesis Kit (Fermentas cat\#K1651) according to manufacturer's protocol. The concentration and quality of the cDNA was determined using a NanoDrop spectrophotometer by measuring the $\mathrm{A}_{260} 6 \mathrm{~A} 280$ ratio and samples were run on a $1.5 \%$ agarose gel in $0.5 \%$ TBE 
buffer to check size and integrity of the synthesized cDNA. The selected gene cluster was targeted with primer combination 3463F835 (5'ATTTTTACGCGGTCTACGC) and 3463 R1036 (5'TGATCAGGTTGCTGTTTCAGG) (Garbeva et al., 2onb) amplifying 202bp from gene Pflo1_3463 encoding the two branched-chain alpha-keto acid dehydrogenase E1 component. From each treatment, 50 ng cDNA was subjected to quantitative RT- PCR using SYBR Green PCR master mix (Applied Biosystem, Warrington, UK). Quantitative RT- PCR was performed on a Corbett Research Rotor- Gene 3000 thermal cycler (Westburg, Leusden, The Netherlands) with the following settings: initial cycle $95{ }^{\circ} \mathrm{C}$ for $15 \mathrm{~min}$, followed by 40 cycles of $95^{\circ} \mathrm{C}$ for $15 \mathrm{sec}, 56^{\circ} \mathrm{C}$ for 50 sec and $72{ }^{\circ} \mathrm{C}$ for $50 \mathrm{sec}$. All analysis was performed in triplicate. Five standard curves $(9.5 \mathrm{ng} / \mu \mathrm{l}, 0.95$ $\mathrm{ng} / \mu \mathrm{l}$, $0.095 \mathrm{ng} / \mu \mathrm{l}$, $0.0095 \mathrm{ng} / \mu \mathrm{l}$ and $0.00095 \mathrm{ng} / \mu \mathrm{l})$ were established. Gene expression data was analyzed with a post-hoc LSD- test and differences between the means of data of different Pseudomonas interactions were considered to be statistically different at $\mathrm{p} \leq 0.05$.

\section{Malthusian parameter}

As an estimate for fitness of the Pseudomonas fluorescens Pfo-1 as monoculture or in competition with the two other strains was calculated by applying the Malthusian parameter (M) growth model (Vasi et al., 1994;van den Berg et al., 2008).

The Malthusian parameter was calculated for both monocultures and mixed cultures by comparing the number of Pseudomonas fluorescens Pfo-1 individuals at an initial time $\left(\mathrm{t}_{\mathrm{o}}\right), \mathrm{N}_{\mathrm{o}}$, to the number of Pseudomonas fluorescens Pfo-1 individuals at a future time $\left(t_{N}\right)$ : $M=\ln \left(N_{t} / N_{o}\right) / t$.

\section{Statistical analysis}

Statistical analyses of the cell counts were performed with IBM SPSS Statistics 20 (IBM, Somers, NY, USA) using one-way ANOVA and post-hoc TUKEY LSD test. Significant differences between the controls (monocultures of the respective bacterial strain) and the treatments are marked with an asterisk ( $\mathrm{p} \leq$ 0.05). 


\section{Results and discussion}

In the present study, we investigated how the interactions between phylogenetically different bacterial strains affect the growth of Pseudomonas fluorescens Pfo-1 in sand microcosms under two different nutrient conditions. Our interests were particularly focused on Pseudomonas fluorescens Pfo-1, as our previous research had shown that Pseudomonas fluorescens Pfo-1 responded differently (behavior and gene expression profile) to phylogenetically different bacteria on nutrient poor agar (Garbeva et al., 2011b).

The growth of Pseudomonas fluorescens Pfo-1 in microcosms supplemented with either nutrient rich or nutrient poor growth media are presented in Figure 5.1A and 5.1B. Bacterial enumeration revealed that all tested bacterial strains used in this study were able to grow in the sand microcosms although with lower numbers under nutrient poor conditions (Figure 5.2A and 5.2B). In microcosms supplemented with nutrient rich media Pseudomonas fluorescens Pfo-1 reached approximately $5.5 * 10^{5}$ cells/g of sand as a monoculture, while in microcosms supplemented with nutrient poor media reached only $9.2 * 10^{4}$ cells/g of sand.

The Bacillus sp. V102 cell numbers in monocultures reached $3.6 * 10^{4}$ cells/g of sand in nutrient rich microcosms and $7{ }^{*} 10^{3}$ cells/g of sand in nutrient poor microcosms. The cell counts of Pedobacter sp. $\mathrm{V}_{4} 8$ as monoculture were approximately $2.3{ }^{*} 10^{6}$ cells/g of sand in nutrient rich and $2.8 * 10^{4}$ cells/g of sand in nutrient poor microcosms (Figure 5.2A and 5.2B). 


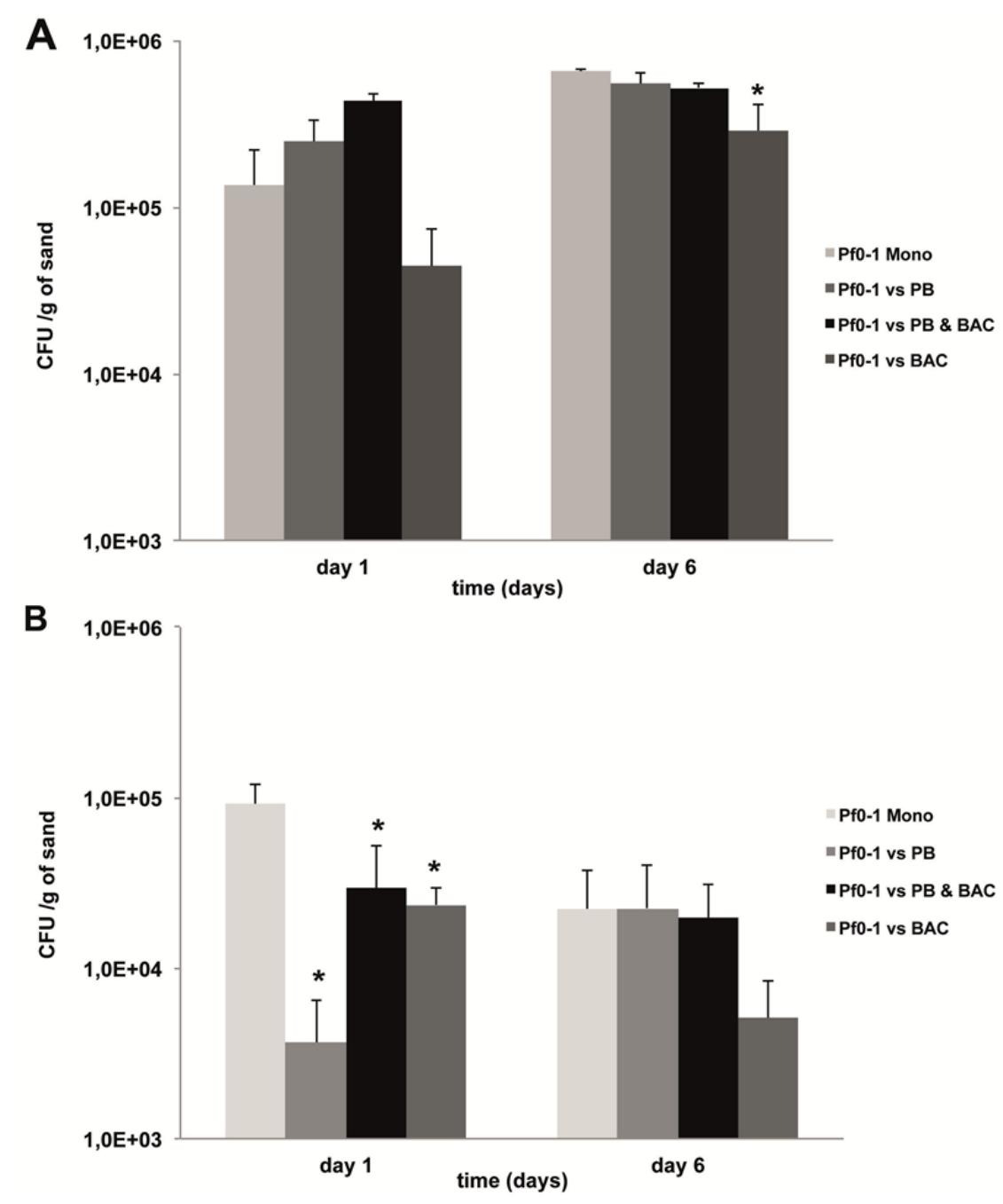

Figure 5.1: Cell counts of Pseudomonas fluorescens Pfo-1 at day 1 and day 6 under (A) nutrient rich and (B) nutrient poor conditions. Significant differences between the numbers of Pfo-1 in monoculture and in mixed cultures are indicated with an asterisk $(\mathrm{p} \leq 0.05)$. Error bars are indicating standard deviation (SD) between the triplicates. Abbreviations: Pfo-1: Pseudomonas fluorescens Pfo-1, PB: Pedobacter sp. V48, BAC: Bacillus sp. V102. 


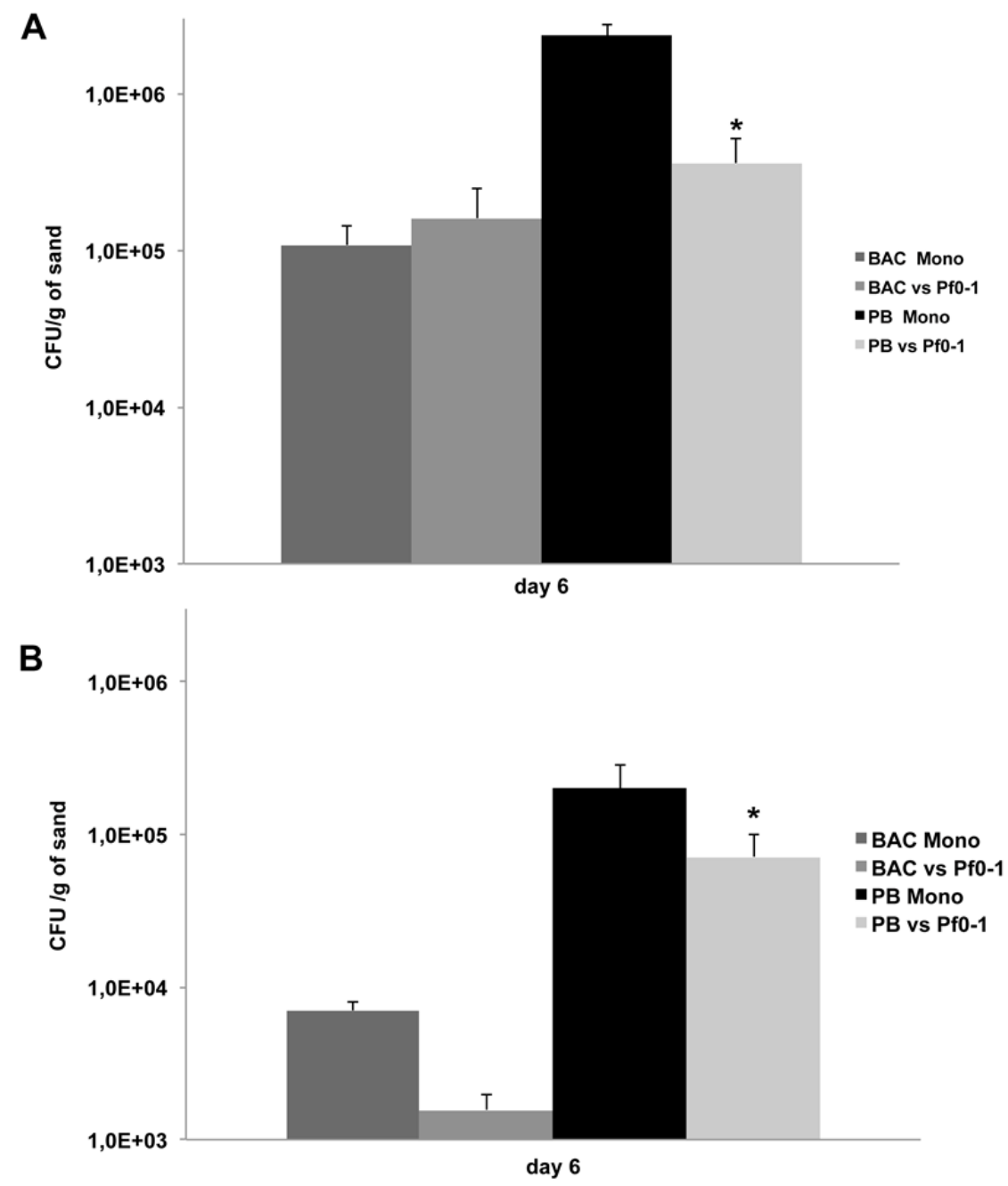

Figure 5.2: Numbers of CFUs of Bacillus sp. V102 and Pedobacter sp. V48 in monoculture and in mixed cultures (with strain Pfo-1) at day 6 under nutrient rich conditions (A) and under nutrient poor conditions (B). Significant differences between the CFUs in monoculture and in mixed cultures are indicated with an asterisk $(\mathrm{p} \leq 0.05)$. Error bars are indicating standard deviation (SD) between the triplicates. Abbreviations: Pfo-1: Pseudomonas fluorescens Pfo-1, BAC: Bacillus sp. V102, PB: Pedobacter sp. V48. 


\section{The effect of phylogenetically different bacteria on the fitness of Pseudomonas fluorescens in sand microcosms}

The growth of Pseudomonas fluorescens Pfo-1 was negatively affected when confronted with the Gram-positive Bacillus sp. V102 strain resulting in significantly lower cell counts at day 6 in nutrient rich microcosms $(\mathrm{p}=0.012)$ and at day 1 in nutrient poor microcosms $(\mathrm{p}=0.008)$. When co-cultivated with Bacillus sp. V102 Pseudomonas fluorescens Pfo-1 reached a maximum of approximately $4.8 * 10^{5}$ cells/g of sand (Figure $5.1 \mathrm{~A}$ and $5.1 \mathrm{~B}$ ). Strong reduction of Pseudomonas fluorescens Pfo-1 growth during confrontation with Bacillus sp. V102 was observed previously on nutrient-poor agar even without direct cell-cell contact (Garbeva et al., 2011b). When co-cultivated with the Gram-negative Pedobacter sp. V48 strain, no significant effect on the growth of Pseudomonas fluorescens Pfo-1 was observed at day $6(\mathrm{p}=0.988)$, whereas there was a significant reduction at day 1 in nutrient-poor microcosms $(\mathrm{p}=\mathrm{o} .000)$. Based on the cell enumeration we applied the Malthusian growth model (population growth) as an estimate for fitness (Figure $\mathbf{S}_{\mathbf{5} .2}$ ). This revealed that the population growth of Pseudomonas fluorescens Pfo-1 was significantly negative affected only during co-cultivation with Bacillus sp. V102 on both day 1 and day $6(\mathrm{p}=0.026$ and $\mathrm{p}=0.014)$.

The observed difference in response of strain Pseudomonas fluorescens Pfo-1 to co-cultivated bacteria was not due to the difference in bacterial growth as both Pedobacter sp. $\mathrm{V}_{4} 8$ and Bacillus sp. V102 were growing in the sand microcosms with Pedobacter sp. $\mathrm{V}_{4} 8$ reaching higher cell counts per gram of sand than Bacillus sp. V102 (Figure 5.2A and 5.2B). However, when cocultivated with both Bacillus sp. V102 and Pedobacter sp. V48 simultaneously, there was no significant effect on the growth of Pseudomonas fluorescens Pfo-1 in both nutrient rich $(\mathrm{p}=0.650)$ and nutrient poor microcosms $(\mathrm{p}=0.995)$ (Figure 5.1A and 5.1B).

From the interspecific interactions investigated in the present study, it is clear that Bacillus sp. V102 acts as "bad" neighbor that can negatively affect the fitness of Pseudomonas fluorescens Pfo-1 as compared to the "good" neighbor Pedobacter sp. $\mathrm{V}_{4} 8$ that did not show any negative effect on the growth of Pseudomonas fluorescens Pfo-1. However, when co-cultivated with both strains simultaneously, Pseudomonas fluorescens Pfo-1 growth was better than when confronted only with Bacillus sp. V102 (Figure 5• 3). 


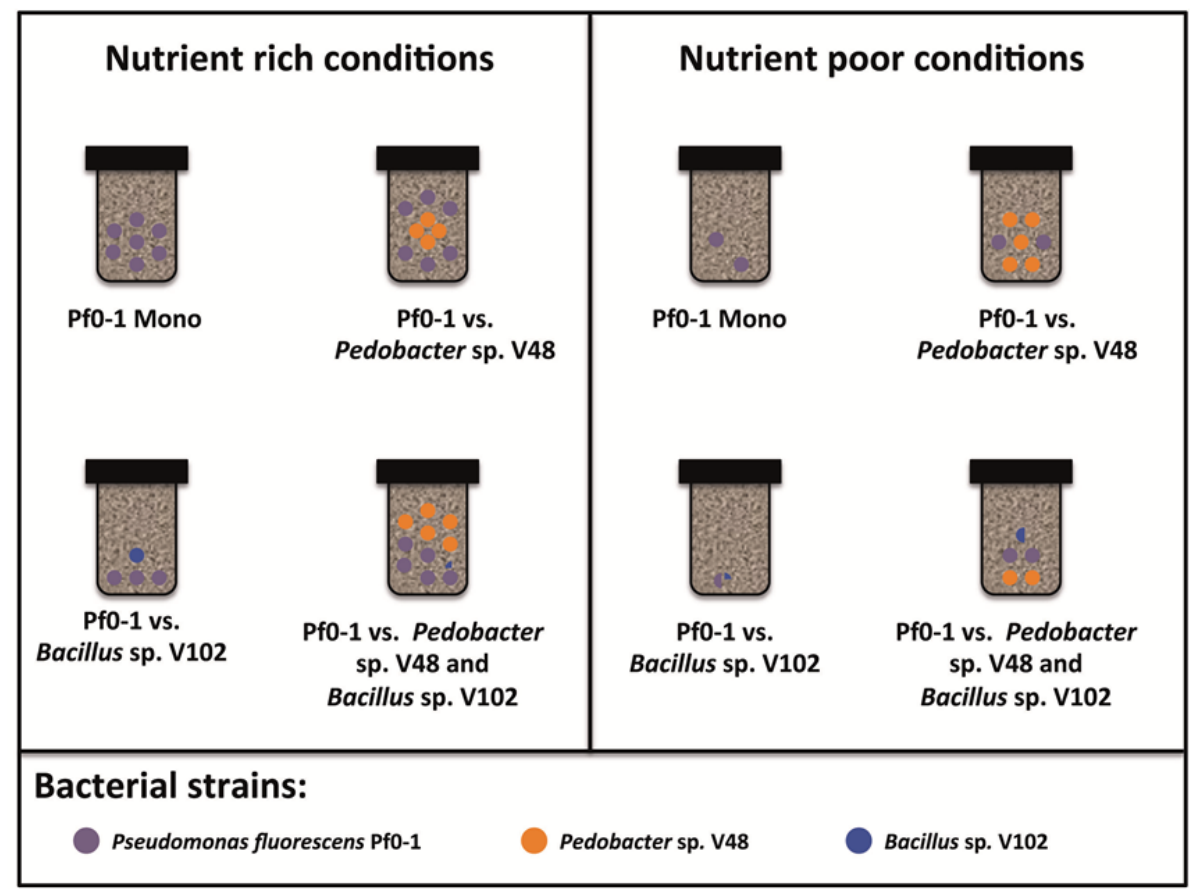

Figure 5.3: Schematic representation of the fitness of Pseudomonas fluorescens Pfo-1 during interspecific interactions with either Pedobacter sp. V48 or Bacillus sp. V102 (2-way interaction) or with both strains together (3way interaction). The number of the coloured circles indicates the effects of the respective interaction on the fitness of Pseudomonas fluorescens Pfo-1. Each full circle represents $1.0 * 10^{5} \mathrm{CFU} / \mathrm{mL}$ (nutrient rich media) or $1.0 * 10^{4}$ $\mathrm{CFU} / \mathrm{mL}$ (nutrient poor media).

From previous studies in our group it is known that Pseudomonas fluorescens Pfo-1 can be triggered to produce a broad-spectrum antibiotic when cocultivated with Pedobacter sp. V48, but not in co-cultivation with Bacillus sp. V102 (Garbeva and de Boer, 2009;Garbeva et al., 2011b). It was hypothesized that this facultative- rather than the constitutive production of antibiotic compound represent a cost-effective strategy, as the antibiotic compound is only produced in situation when it is needed (Garbeva et al., 2011c). 
It is plausible that in a more complex habitat, the production of a broadspectrum antibiotic triggered by Pedobacter sp. $\mathrm{V}_{4} 8$ gives Pseudomonas fluorescens Pfo-1a advantage when confronted with phylogenetically different strains simultaneously.

To confirm that the observed results are related to antibiotic production, we performed quantitative RT-PCR with primers targeting genes Pflo1_3463 known to be involved in the production of a broad-spectrum antimicrobial compound (Garbeva et al., 2011b). Results revealed that indeed genes Pflo1_3463 were highly expressed at day 6 in the microcosms where Pseudomonas fluorescens Pfo-1 was interacting with Pedobacter sp. $\mathrm{V}_{4} 8$ $(\mathrm{p}=0.014)$. Gene expression was slightly higher in treatments were Pseudomonas fluorescens Pfo-1 was confronted with both Pedobacter sp. $\mathrm{V}_{4} 8$ and Bacillus sp. V102, although not significantly $(\mathrm{p}=\mathrm{0.750}$ ) (Figure 5.4). Unfortunately, due to the low cell number in the microcosms supplemented with nutrient poor growth media, our attempts to extract good quality and quantity of RNA for cDNA synthesis and quantitative RT-PCR failed.
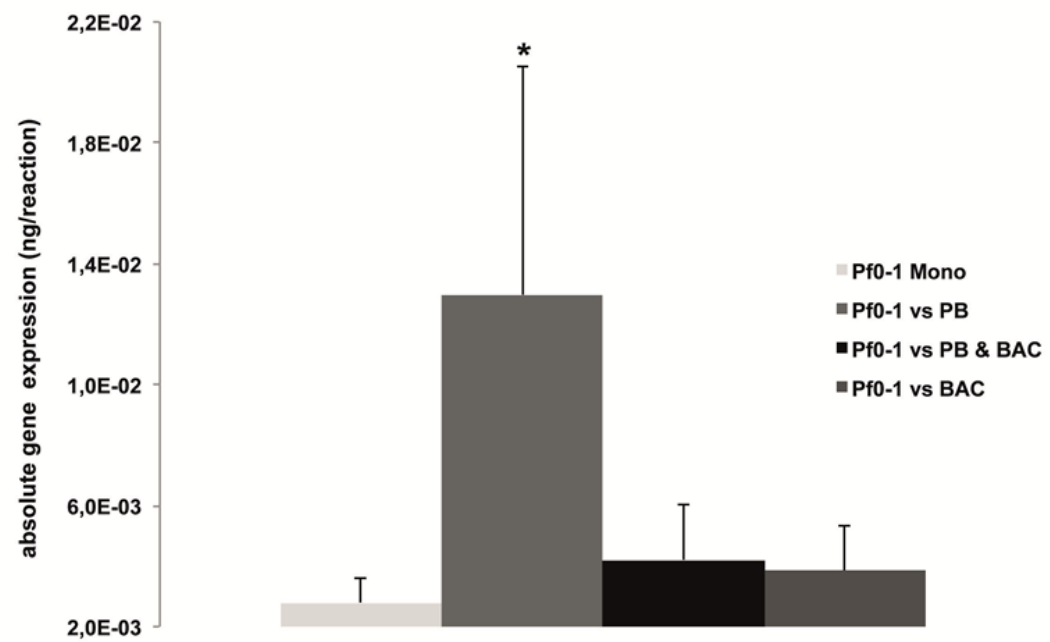

Figure 5.4: qRT-PCR results representing absolute gene expression of gene cluster Pfo-1_3463 obtained at day 6 (nutrient rich media). Error bars are indicating standard deviation (SD) between the triplicates. Significant differences between the qRT-PCR based gene expression by Pfo-1 in monocultures and mixed cultures is indicated by an asterisk ( $\mathrm{p} \leq 0.05)$. 
Interspecific interactions may trigger the production of antimicrobial compounds in complex microbial communities where this so-called chemical warfare may offer comparative advantage for the producing strains (Hibbing et al., 2010;Foster and Bell, 2012). A recent study showed that interspecific interactions between soil bacteria can have a major impact on antimicrobial compound production with effects in both directions, i.e. induction or suppression of antimicrobial compound production (Tyc et al., 2014). In soil and in the rhizosphere environment Pseudomonas species coexist with many other bacterial species and compete for the same nutrient resources (Demoling et al., 2007;Jousset et al., 2011;Becker et al., 2012;Inglis et al., 2012). The ability to cope with the presence of a range of competing microbial species is essential for growth and survival in soil ecosystems and the performance of soil bacteria may strongly depend on the neighboring competitors.

Overall, our data suggests that the performance of Pseudomonas fluorescens Pfo-1 in sand microcosms depends greatly on the presence and identity of neighboring microorganisms. Although Pseudomonas fluorescens Pfo-1 cell counts were lower in the nutrient poor sand microcosms than in the nutrient rich microcosms, similar growth patterns were observed in both experiments. This indicates that, contrary to our initial hypothesis, nutrient levels did not have a strong effect on multispecies interactions and on the ability of Pseudomonas fluorescens Pfo-1 to respond to different bacteria. It is well known that under different nutrient conditions bacteria often produce different secondary metabolites (Sanchez et al., 2010;van Wezel and McDowall, 2011;Garbeva et al., 2014b) and hence influence microbial interactions in different ways.

This work demonstrates that interspecific interactions can play an important role in soil and may influence microbial performance and consequently shape the composition of microbial communities.

\section{Acknowledgment}

This work was supported by The Netherlands Organization for Scientific Research (NWO) MEERVOUD personal grant issued to Paolina Garbeva (836.09.004). The authors want to thank Cristina Martinez Romera for her help during the experiment. We thank Professor Wietse de Boer for critical reading of this manuscript and his valuable and constructive comments. This is publication 5773 of the NIOO-KNAW. 


\section{Supplementary material}

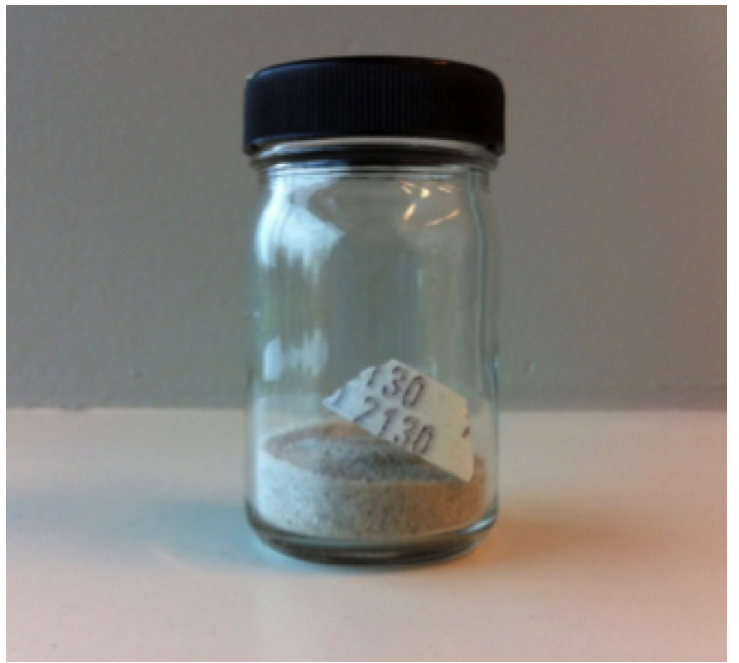

Figure S5.1: Example of a sand microcosm used in this study.

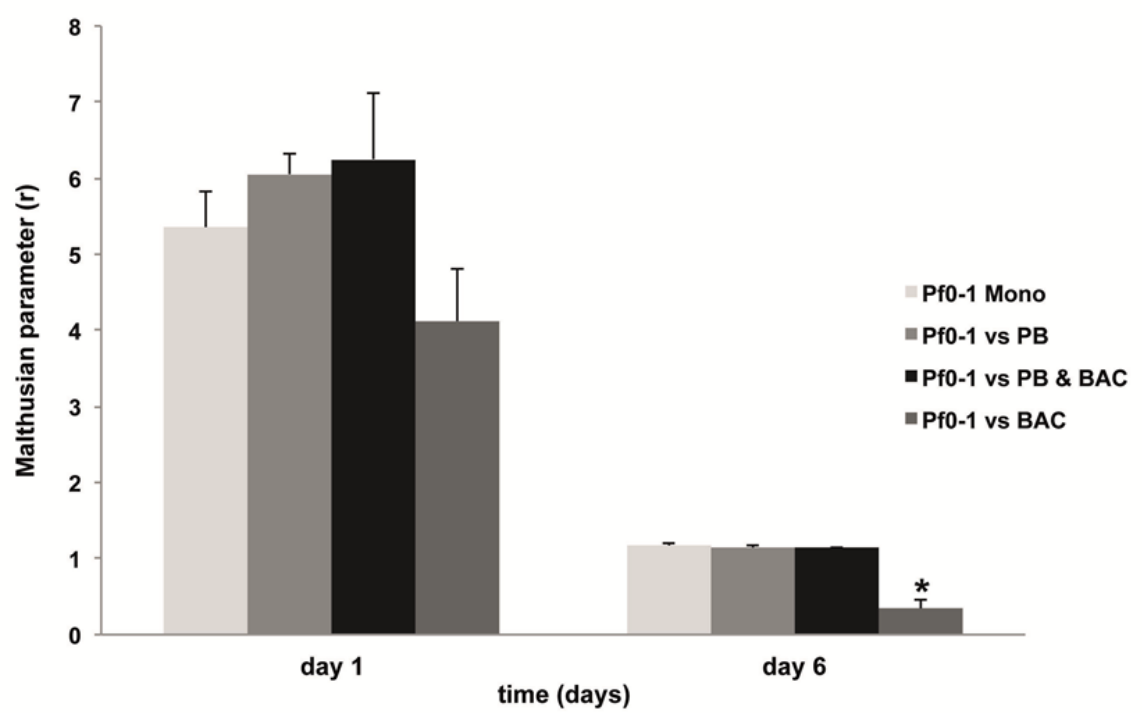

Figure S5.2: Malthusian parameter calculated for the time interval from day o to day 1 and for the time interval from day o to day 6 (nutrient rich media) representing the fitness of Pseudomonas fluorescens Pfo-1 in the four different microcosm treatments. Error bars are indicating standard deviation (SD) between the triplicates. Significant differences are indicated by an asterisk ( $\mathrm{p} \leq 0.05)$. 


\section{Chapter}

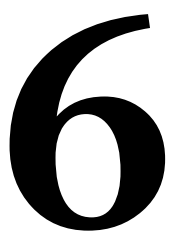

\section{No apparent costs for facultative antibiotic production by the soil bacterium Pseudomonas fluorescens Pfo-1}

Paolina Garbeva, Olaf Tyc, Mitja N.P. Remus-Emsermann, Annemieke van der Wal, Michiel Vos, Mark Silby and Wietse de Boer

This chapter has been published as:

Garbeva P, Tyc O, Remus-Emsermann MNP, van der Wal A, Vos M, Silby M et al (2011). No Apparent Costs for Facultative Antibiotic Production by the Soil Bacterium Pseudomonas fluorescens Pfo-1. PLoS One 6 doi: 10.1371/journal.pone.0027266 


\begin{abstract}
Background: Many soil-inhabiting bacteria are known to produce secondary metabolites that can suppress microorganisms competing for the same resources. The production of antimicrobial compounds is expected to incur fitness costs for the producing bacteria. Such costs form the basis for models on the co-existence of antibiotic-producing and non-antibiotic producing strains. However, so far studies quantifying the costs of antibiotic production by bacteria are scarce. The current study reports on possible costs, for antibiotic production by Pseudomonas fluorescens Pfo-1, a soil bacterium that is induced to produce a broad-spectrum antibiotic when it is confronted with non-related bacterial competitors or supernatants of their cultures.
\end{abstract}

Methodology and Principal Findings: We measured the possible cost of antibiotic production for Pseudomonas fluorescens Pfo-1 by monitoring changes in growth rate with and without induction of antibiotic production by supernatant of a bacterial competitor, namely Pedobacter sp.. Experiments were performed in liquid as well as on semi-solid media under nutrientlimited conditions that are expected to most clearly reveal fitness costs. Our results did not reveal any significant costs for production of antibiotics by Pseudomonas fluorescens Pfo-1. Comparison of growth rates of the antibioticproducing wild-type cells with those of non-antibiotic producing mutants did not reveal costs of antibiotic production either.

Significance: Based on our findings we propose that the facultative production of antibiotics might not be selected to mitigate metabolic costs, but instead might be advantageous because it limits the risk of competitors evolving resistance, or even the risk of competitors feeding on the compounds produced. 


\section{Introduction}

Interference competition is an important strategy of bacterial strains to establish and maintain themselves within microbial communities (Hibbing et al., 2010). A well-known mechanism of bacterial interference competition is the production of antibiotics (Raaijmakers et al., 2002).These secondary metabolites can be targeted against more or less closely related strains and species (e.g. bacteriocins) or against a wide range of competitors (e.g. many polyketides) (Riley and Wertz, 2002a;Challis and Hopwood, 2003;Riley et al., 2003). Theoretical models have demonstrated that, instead of decreasing diversity by leaving only the most aggressive strains, microbial warfare could actually promote diversity, with dynamic coexistence of many strains differing in their antibiotic production and sensitivity profiles (Czaran and Hoekstra, 2003; Gardner and West, 2004;Czaran and Hoekstra, 2007;Hibbing et al., 2010). These results are obtained when it is assumed that both resistance to- and production of antibiotics come at a fitness cost, resulting in a reduced growth rate. The ecological trade-offs involved in investment in killing, resisting or outgrowing competing strains is thus predicted to maintain diversity.

Because of its profound relevance to human health, the fitness cost of bacterial resistance to antibiotics has received far more attention than the fitness cost of bacterial antibiotic production. Whereas it has emerged that the majority of bacterial antibiotic resistance mechanisms comes at a fitness cost (Andersson and Hughes, 2010), as predicted by theory (Coustau et al., 2000), few studies have examined the cost of antibiotic production. Indications for biological costs of antibiotic production or antibiotic resistance are generally obtained by comparison of relative fitness of wild-type strains with that of antibiotic-negative mutant strains (e.g. (Ender et al., 2004;Binet and Maurelli, 2005)). However, mutations causing loss of antibiotic production may cause additional changes in the bacterial phenotype (Bjorkman et al., 1998;Bjorkman and Andersson, 2000).

Previously, we have reported on competitor-induced triggering of broadspectrum antibiotic production in fluorescent pseudomonads (Garbeva and de Boer, 2009;Garbeva et al., 2011b). The soil isolate Pseudomonas fluorescens Pfo-1 exhibits antibiotic activity only when it is confronted with specific phylogenetically unrelated competitors (e.g. Pedobacter sp.) or their supernatant indicating that it can distinguish interspecific competition from intraspecific competition (Garbeva et al., 2011b). Although the structure of the antimicrobial compound has not yet been elucidated, upregulated genes 
during confrontation with competitors point at the synthesis of a polyketidelike compound (Garbeva et al., 2011b). In addition, we have shown that it has broad-spectrum activity, acting against both Gram-positive and Gramnegative bacteria as well as against fungi (Garbeva et al., 2011b).

Competitor-dependent induction of antibiotic production allows for another possibility to examine costs of antibiotic production namely by comparing growth rates of wild-type bacteria with and without induction of antibiotic production. In the current study we used both approaches to quantify the possible fitness cost of antibiotic production in $P$. fluorescens Pfo1: 1) comparison of the growth rate of the wild-type with and without induction of antibiotic production and 2) comparison of the growth rate of wild-type and non-antibiotic producing mutants under conditions that induce antibiotic production. All experiments were performed using nutrient-poor media, as soil-dwelling bacteria typically experience a scarcity of easily degradable carbon resources (Alden et al., 2001;Demoling et al., 2007). Moreover, growth limiting conditions represent a situation in which the costs of antibiotic production should be most pronounced as there is no surplus of energy resources (Anderl et al., 2003). Costs were measured in liquid culture as well as on semi-solid medium in an incubation chamber, allowing quantification of micro-colony growth. 


\section{Materials and methods}

\section{Bacterial and fungal cultures used}

The bacterial strains used in this study are Pseudomonas fluorescens Pfo-1 (Gamma-proteobacteria) which was isolated from an agricultural loam soil in Sherborn, Massachusetts, USA (Compeau et al., 1988) and Pedobacter sp. V48 (Sphingobacteria), which was isolated from a coastal dune site in The Netherlands (de Boer et al., 2007). The strains were pre-cultured from frozen glycerol stocks on 1/10 strength Tryptic Soy Broth agar (TSBA) (Garbeva and de Boer, 2009). In addition to the parental strain, deletion mutant $\Delta$ Pflo1_3463-66 (with deletion of Pflo1_3463-3464: two branched-chain alphaketo acid dehydrogenase E1 components; Pflo1_3465: branched-chain alphaketo acid dehydrogenase subunit E2 and Pflo1_3466: dihydrolipoamide dehydrogenase) which is unable to produce the broad-spectrum antibiotic was used as well (Garbeva et al., 2011b). The fungal isolate Rhizoctonia solani anastomosis group 2.2IIIB, a plant-pathogenic basidiomycete, was used as bioindicator for production of broad-spectrum antimicrobial compounds (Garbeva et al., 2011b).

\section{Preparation of cell-free Pedobacter supernatant}

Cell-free supernatant was prepared by centrifugation (16,0oo x g for $5 \mathrm{~min}$ ) followed by filtration (Spin-X $0.22 \mu \mathrm{m}$ filters; Corning Costar, Cat\# 8160) of over-night cultures of Pedobacter strain $\mathrm{V}_{4} 8$ grown in $1 / 10^{\text {th }}$ strength Tryptic Soy Broth at $20{ }^{\circ} \mathrm{C}$. An aliquot of the cell-free supernatant was boiled for 10 minutes and was used as a control. Boiling the cell-free supernatant for 10 minutes was sufficient to destroy the signalling compound(s) that trigger antibiotic production in $P$. fluorescens Pfo-1.

\section{Determination of growth of Pfo-1 in nutrient-poor liquid media}

The effect of cell-free supernatant from Pedobacter sp $\mathrm{V}_{4} 8$ on the growth rate of Pfo-1 was determined in a nutrient-poor liquid medium (0.5 gL $\mathrm{gL}_{2}^{-1} \mathrm{KO}_{4}$; $0.1 \mathrm{gL}^{-1} \mathrm{BD}$ Bacto ${ }^{\mathrm{TM}}$ Yeast extract (Cat\# 210934) and $0.1 \mathrm{gL}^{-1}\left(\mathrm{NH}_{4}\right)_{2} \mathrm{SO}_{4}$; adjusted to $\mathrm{pH}$ 6.5). This medium was supplemented with $10 \%(\mathrm{v} / \mathrm{v}$ ) boiled (control) or unboiled Pedobacter supernatant, respectively. P. fluorescens Pfo-1 was inoculated to an optical density (OD) $(600 \mathrm{~nm})$ of 0.02 which corresponds to $2.6 \times 10^{6} \mathrm{cells} / \mathrm{ml}^{-1}$.

The $50 \mathrm{ml}$ cultures were incubated for 24 hours at $20{ }^{\circ} \mathrm{C}$ shaken at $200 \mathrm{rpm}$ and $1 \mathrm{ml}$ samples were taken every hour for OD measurements and viable counts. Additionally, the growth rate of P. fluorescens Pfo-1 wild-type and 
mutants $\Delta$ Pflo1_3463 (both supplemented with $10 \%(\mathrm{v} / \mathrm{v})$ boiled (control) or unboiled Pedobacter supernatant) were measured in 96-well plates (Greiner bio-one, Cat\# 655180) using Synergy Microplate Reader. The OD measurements were performed every 30 minutes for total period of 8.5 hours.

\section{Agar-incubation chambers for observation of bacterial growth on semi- solid medium}

$1 \mathrm{ml}$ of $P$. fluorescens Pfo-1 overnight culture was centrifuged for $3 \mathrm{~min}$ at 16,ooo g. The cells were resuspended in 1omM phosphate buffer ( $\mathrm{pH} 6.5$ ) containing $10 \%$ non-boiled or boiled cell-free supernatant of Pedobacter sp $\mathrm{V} 48$ to an optical density of 0.002 at $600 \mathrm{~nm}$; so, the initial number of cells per $\mathrm{ml}$ was 10 times lower than in the liquid growth experiments. The lower number was used as it allows distinguishing individual bacteria on the agar slices (see below). An aliquot of $280 \mu \mathrm{l}$ of P. fluorescens Pfo-1 bacterial suspension was equally spread on a thin layer $(2 \mathrm{~mm})$ of water agar containing $5 \mathrm{~g} \mathrm{~L}^{-1} \mathrm{NaCl}$; $1 \mathrm{~g} \mathrm{L-1} \mathrm{KH}_{2} \mathrm{PO}_{4}$ and $0.1 \mathrm{~L}-1\left(\mathrm{NH}_{4}\right)_{2} \mathrm{SO}_{4}$ adjusted to $\mathrm{pH} 6.5$ as previously described (Garbeva and de Boer, 2009). Since bacterial growth was observed on this water-agar medium no extra substrate (yeast extract) was added (Garbeva et al., 2011b). Apparently, the substrates that were added with the $10 \%$ Pedobacter supernatant and the substrates that became available after autoclaving of agar were sufficient for growth. After inoculation the plates were dried for about 5 minutes in a flow cabinet and 3 slices of $1 \mathrm{~cm}^{2}$ were cut and carefully transferred to incubation chambers. The incubation chamber described in Figure 6. 1 is adapted from Reinhard and Van der Meer (Reinhard and Van der Meer, 2011) and Robin Tecon (unpublished data).

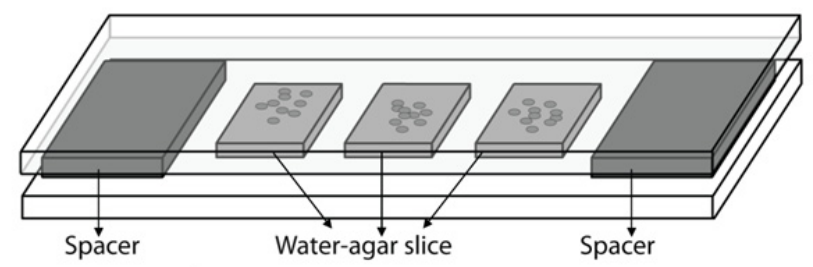

Figure 6.1: Incubation chamber for determination of bacterial growth made of a glass slide with cardboard spacers on both sides. Between the spacers 3 pre-inoculated water-agar (WA) slices of $1 \mathrm{~cm}^{2}$ are placed. A glass coverslip is slightly pressed on top of the agar slices. The sides of the incubation chamber are sealed with parafilm. 


\section{Microscopical counting and data analysis}

Twenty randomized pictures at 40o-fold magnification representing an area of $1 \mathrm{~mm}^{2}$ were taken of each slice with an Axio Imager Mi microscope (Carl Zeiss, Oberkochen, Germany) under phase-contrast illumination and an AxioCam MRm camera. Microscopic enumerations were performed every hour for a period of 9 hours at room temperature. During this period microcolonies remained two-dimensional, i.e. no stacking took place. Digital images were analyzed using the AXIO VISION v4.7 Software (Carl Zeiss Imaging Solutions $\mathrm{GmbH}$, Germany) for enumeration and area determination of bacterial colonies.

\section{Extraction of antimicrobial compound and test for inhibition}

P. fluorescens Pfo-1 overnight liquid cultures exposed to $10 \%$ boiled or unboiled supernatant of Pedobacter sp $\mathrm{V}_{4} 8$ (initial volume $35 \mathrm{ml}$ ) were acidified with trifluoroacetic acid (o.1 \% (v/v)), mixed with 2 volumes of ethylacetate and shaken vigorously for $5 \mathrm{~min}$. as described by Raaijmakers et al. (Raaijmakers et al., 1999). After overnight incubation at $-20{ }^{\circ} \mathrm{C}$ the unfrozen liquid (ethylacetate) fraction containing the active compounds was carefully transferred to a new flask and dried under constant air-flow. The dried extract was dissolved in $150 \mu \mathrm{l} 50 \%(\mathrm{v} / \mathrm{v})$ methanol and tested for inhibition of the bacterial isolate Pedobacter sp $\mathrm{V}_{4} 8$ and the soil borne plantpathogenic fungus Rhizoctonia solani as described by Garbeva et al. (Garbeva et al., 2011b).

\section{RNA extraction, cDNA synthesis and real-time PCR}

RNA was extracted from $P$. fluorescens Pfo-1 grown in nutrient-poor liquid medium that had been exposed to $10 \%$ boiled or non-boiled supernatant of Pedobacter sp $\mathrm{V}_{4} 8$ as described above. Cells for RNA extraction were collected at 4 time points ( $3 \mathrm{~h}, 6 \mathrm{~h}, 8 \mathrm{~h}$ and $24 \mathrm{~h}$ ) and diluted with sterile phosphate buffer to the same optical density (6oonm) to obtain equal amounts of cells. The RNA extraction and cDNA synthesis were performed as described previously by Garbeva et al (Garbeva et al., 2011b). For real-time PCR assessment of expression of genes involved in antibiotic production two different primer combinations were used: (1) primer combination 3463F835 (GAT TTT TAC GCG GTC TAC GC) and 3463Rio36 (TGA TCA GGT TGC TGT TTC AGG) amplifying 206bp from gene Pfo1_3463 encoding the two branched-chain alpha-keto acid dehydrogenase E1 component and (2) primer combination 3465TiF (CAG GGC CCG ATG GTT GC) and 3465TiR (TTG CTT TTT GTG CCG CGC TCG) amplifying 348bp from gene Pfo1_3465 encoding branched- 
chain alpha-keto acid dehydrogenase subunit E2. As a control, a 210bp fragment of the house keeping $16 \mathrm{~S}$ rDNA gene was amplified using primer combination 16SPfoiF (TTG GGA GCC TTG AGC TCT TA) and 16SPforR (AAG GCA CCA ATC CAT CTC TG). Real-time PCR was performed using a Corbett Research Rotor-Gene 3000 thermal cycler (Westburg, Leusden, The Netherlands) with the following conditions: an initial cycle of $95^{\circ} \mathrm{C}$ for $15 \mathrm{~min}$ followed by 40 cycles of: $95^{\circ} \mathrm{C}$ for $15 \mathrm{sec} ; 56^{\circ} \mathrm{C}$ for 5 osec and $72^{\circ} \mathrm{C}$ for $50 \mathrm{sec}$. Standard curves were established for each primer combination.

\section{Statistical analysis}

All experiments were performed in triplicate. Differences in optical densities of liquid cultures between treatments were tested for significance for each time interval by one-way analysis of variance. Bacterial viable count data that were used to calculate the maximum yield (maximum number of colony forming units per $\mathrm{ml}$ ) were also log-transformed prior to one-way analysis of variance. For the analysis of microcolony development the data were $\log$ transformed and the slopes of the regression lines were compared in GraphPad Prism 5 (GraphPad Software, Inc., CA, USA) using a two-tailed ttest. The statistical analyses of quantitative real-time PCR data were carried out with XLStat 2010 (Addinsoft, New York, USA) using a two-tailed t-test. Data were considered to be statistically different at $\mathrm{p} \leq 0.05$. 


\section{Results}

\section{$P$. fluorescens Pfo-1 antibiotic production and growth rate}

The OD measurements indicated that Pfo-1 grew only a short period $(1-2 \mathrm{hr})$ exponentially in nutrient-poor liquid media followed by linear increase (Figure 6.2, Figure 6.3, Figure S6.1). This rapid decline in growth rates occurred for all treatments and stationary phase was reached after 8 hours. At none of the time intervals (performed on 96-well plates every $30 \mathrm{~min}$ ) were there significant differences in OD between cultures exposed to boiled and unboiled Pedobacter supernatant (Figure 6. 2). The growth rate of Pfo-1 mutant $\Delta_{3463}$, which is deficient in the production of the broad-spectrum antimicrobial compound triggered by Pedobacter supernatant, was also compared with that of the wild-type strain in the presence of Pedobacter supernatant. Again, no differences in OD were observed (Figure 6.2).

Similar observations were made for the $50 \mathrm{ml}$ cultures where OD measurements were performed every hour (Figure S6.1). For the latter cultures, the maximum yield (highest number of viable cells per $\mathrm{ml}$ of growth medium) was calculated and again no differences were found between the treatments (Table 6.1.).

Table 6.1: Maximum yield of $P$. fluorescens Pfo-1 wild-type and mutant $\Delta_{3463}$ strains in nutrient-poor liquid medium.

\begin{tabular}{|c|c|}
\hline Treatment & Maximum yield * (CFU per $\mathrm{ml})$ \\
\hline $\begin{array}{l}\text { Pfo-1 wild-type }+10 \% \text { cell-free } \\
\text { Pedobacter supernatant } \\
\text { Pfo-1 wild-type }+10 \% \text { boiled cell-free } \\
\text { Pedobacter supernatant } \\
\Delta 3463+10 \% \text { cell-free Pedobacter } \\
\text { supernatant } \\
\Delta 3463+10 \% \text { boiled cell-free Pedobacter } \\
\text { supernatant }\end{array}$ & $\begin{array}{l}7.99 \pm 0.013 \\
7.96 \pm 0.022 \\
8.00 \pm 0.021 \\
7.99 \pm 0.016\end{array}$ \\
\hline
\end{tabular}

* The maximum yield calculated at time point $=7.5 \mathrm{~h}$ is the mean of tree replicates per treatment. The variation between the replicates is indicated as \pm s.d.

One-way ANOVA did not reveal difference between the treatments p>0.05. 
The liquid cultures used for growth rate determinations were extracted at the end of the incubation to confirm that the wild-type Pfo-1 was induced by Pedobacter supernatant to produce antibiotics. The presence of antibiotic activity in the extracts was tested by determining the effect of the extracts on growth of Pedobacter sp $\mathrm{V}_{4} 8$ and the soil-borne pathogenic fungus Rhizoctonia solani. Inhibition of Rhizoctonia and Pedobacter was observed only with the extracts from wild type Pfo-1 cultures exposed to $10 \%$ cell-free Pedobacter supernatant (Figure S6.2A, B). There was no such inhibition by the extracts obtained from Pfo-1 cultures exposed to $10 \%$ boiled cell-free Pedobacter supernatant or from Pfo-1 mutant $\Delta 3463$ cultures exposed to $10 \%$ cell-free Pedobacter supernatant.

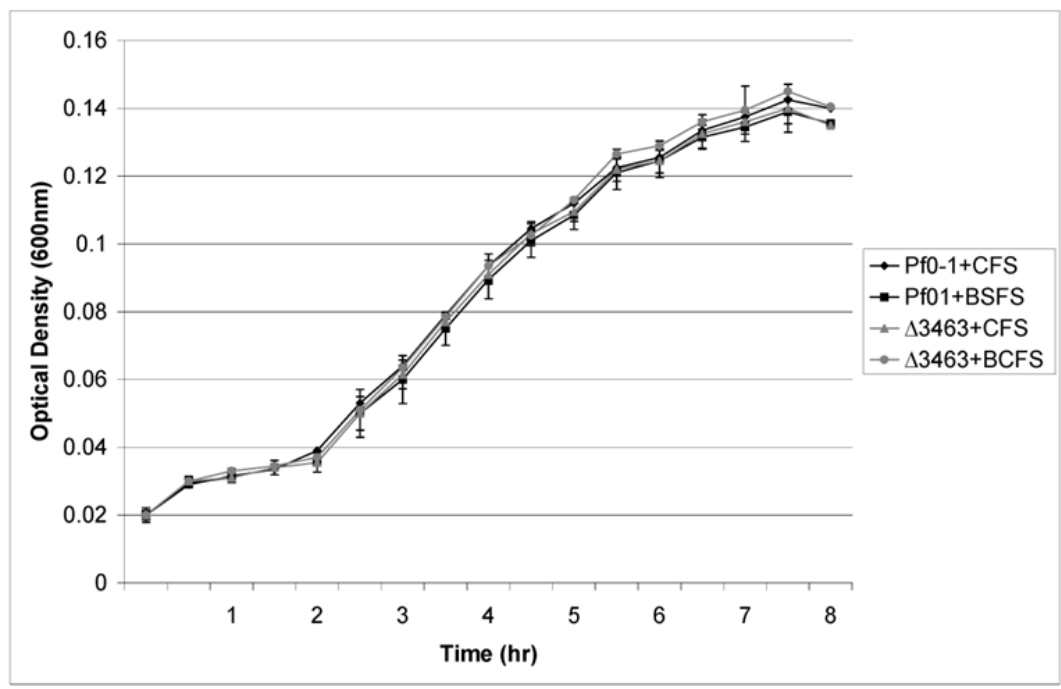

Figure 6.2: Bacterial growth (Optical Density at $600 \mathrm{~nm}$ ) in nutrient-poor liquid medium in microplates: Pforwt+CFS - P. fluorescens Pfo-1 wild type with $10 \%$ cell-free supernatant from Pedobacter sp. V48; Pforwt+BCFS P. fluorescens Pfo-1 wild type with $10 \%$ boiled cell-free supernatant from

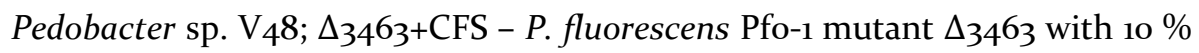
cell-free supernatant from Pedobacter sp. $\mathrm{V}_{4} 8$ and $\Delta_{3463+\mathrm{BCFS}}-$ $P$. fluorescens Pfo-1 mutant $\Delta 3463$ with $10 \%$ boiled cell-free supernatant from Pedobacter sp. $\mathrm{V}_{4} 8$. The measurements were performed every $30 \mathrm{~min}$ for total period of $8.5 \mathrm{~h}$. Symbols represent means of 3 replicate measurements; error bars represent standard deviations. 


\section{Expression of genes involved in antimicrobial compound production}

At four time points $(\mathrm{t}=3 \mathrm{~h}, 6 \mathrm{~h}, 8 \mathrm{~h}$ and $24 \mathrm{~h})$ during growth in liquid medium, $P$. fluorescens Pfo-1 cells were collected for RNA isolation, cDNA synthesis and quantitative RT-PCR. Primers targeting genes Pflo1_3463 and Pflo1_3465 were used for quantitative RT-PCR. Genes Pflo1_3463 and Pflo1_3465 encode branch-chain alpha-keto acid dehydrogenase E1 components and branchedchain alpha-keto acid dehydrogenase subunit E2 that were previously demonstrated to be essential for the production of broad-spectrum antimicrobial activity by $P$. fluorescens Pfo-1 (Garbeva et al., 2011b). Quantitative RT-PCR revealed that triggering of Pflo1_3463 and Pflo1_3465 genes expression by Pedobacter supernatant was already apparent after three hours, as there was a significant (3.16 and 2.57 fold respectively) increase in comparison with the expression of these genes in cultures of Pfo-1 exposed to $10 \%$ boiled Pedobacter supernatant (Table 6.2). The expression of genes Pflo1_3463 and Pflo1_3465 was always at least two-fold higher in the Pfo-1 cultures exposed to $10 \%$ cell-free Pedobacter supernatant than in the controls (Pfo-1 cultures exposed to $10 \%$ boiled cell-free Pedobacter supernatant).

Table 6.2: Quantitative real-time PCR comparison of gene expression in $P$. fluorescence Pfo-1 with triggered antibiotic production (treatment) and non-triggered antibiotic production (control).

\begin{tabular}{c|c|c}
\hline $\begin{array}{c}\text { Time points } \\
\text { hours }\end{array}$ & $\begin{array}{c}\text { Fold change treatment/ to } \\
\text { control gene Pfo1_3463 }\end{array}$ & $\begin{array}{c}\text { Fold change treatment/ to } \\
\text { control gene } P \text { for_3465 }\end{array}$ \\
\hline 3 & $3.16 \pm 0.03$ & $2.57 \pm 0.11$ \\
6 & $2.39 \pm 0.06$ & $2.68 \pm 0.03$ \\
8 & $2.11 \pm 0.11$ & $3.21 \pm 0.14$ \\
24 & $2.50 \pm 0.10$ & $2.6 \pm 0.10$
\end{tabular}

Differential expression is given as fold-changes treatment relative to the control.

The numbers are the means of tree replicates per treatment. The variation between the replicates is given as \pm s.d. All fold changes of gene expression in the treatment were significantly different $(\mathrm{p}<0.05)$ from the control. 
Effect of antibiotic production on P. fluorescens Pfo-1 growth rate on agar and microcolony morphology

Using water-agar incubation chambers we determined growth rate and size of Pfo-1 colonies with and without induction of antibiotic production by Pedobacter supernatant. Growth (increase of surface areas micro-colonies) was exponential during the period of examination (Figure 6. 3). There was no significant difference in growth rates between the two treatments (Figure 6. 3A). No significant differences in growth rates were observed between the colonies of wild type and mutant $\Delta_{3463}$ strains either (Figure 6 . $\left.{ }_{3} \mathbf{B}\right)$. In fact, the average colony size of Pfo-1 supplied with $10 \%$ cell-free supernatant from Pedobacter sp $\mathrm{V}_{4} 8$ was slightly (but not significantly) bigger than that of Pfo-1 supplied with boiled cell-free supernatant. However, there was a clear difference in colony morphology. After 7 hours of incubation, wild-type Pfo-1 exposed to 10 \% Pedobacter supernatant started to form spherical colonies, a phenomenon not observed for Pfo-1 exposed to boiled Pedobacter supernatant or for Pfo-1 mutant $\Delta_{3463}$ exposed to unboiled Pedobacter supernatant (Figure 6. 4).
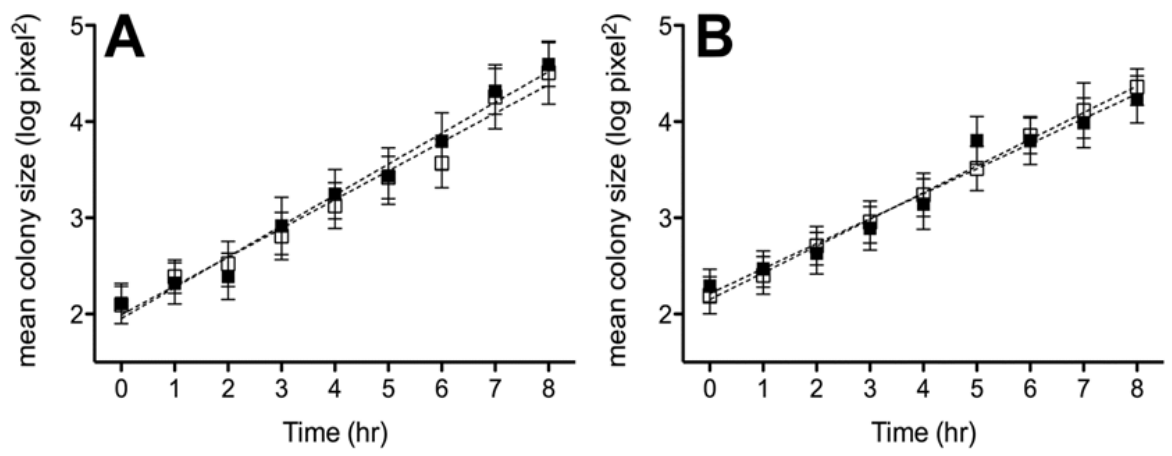

Figure 6.3: Bacterial growth in micro-colonies measured microscopically using Water-Agar incubation chambers (A) white squares -wild type P. fluorescens Pfo-1 exposed to $10 \%$ cell-free supernatant from Pedobacter sp. $\mathrm{V}_{4} 8$ and black squares- wild type $P$. fluorescens Pfo-1 exposed to $10 \%$ boiled cell-free supernatant from Pedobacter sp. V48; (B) white squares -wild type $P$. fluorescens Pfo-1 exposed to $10 \%$ cell-free supernatant from Pedobacter sp. $\mathrm{V}_{4} 8$ and black squares- mutant $\Delta 3463$ exposed to $10 \%$ cell-free supernatant from Pedobacter sp. V48. Squares represent the means of micro-colony sizes and the error bars represent the standard deviations. Statistical analysis revealed no significant differences $(\mathrm{p}>0.05)$ between the growth rates (slopes) of the treatments. 


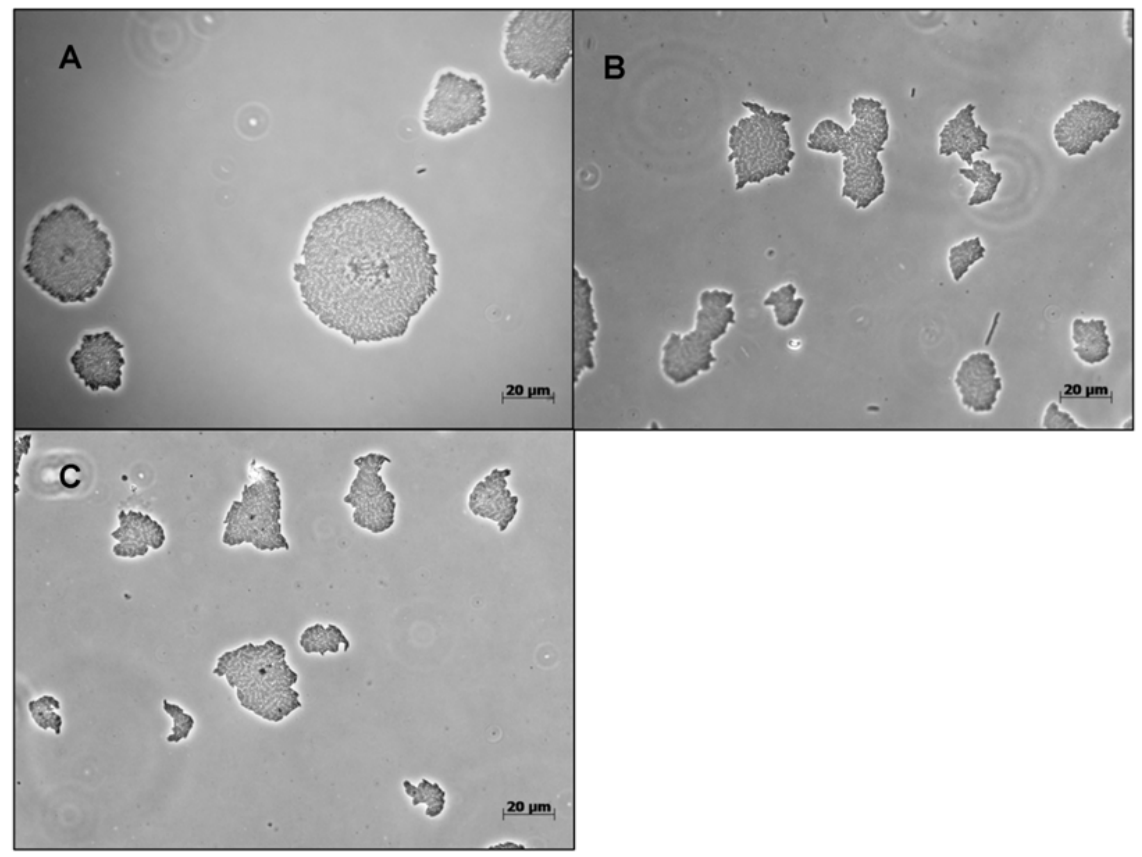

Figure 6.4: Morphology of bacterial micro-colonies after 7 h of incubation on Water-Agar of (A) wild type P. fluorescens Pfo-1 exposed to $10 \%$ cell-free supernatant from Pedobacter sp. $\mathrm{V}_{48} 8$ (B) wild type P. fluorescens Pfo-1 exposed to $10 \%$ boiled cell-free supernatant from Pedobacter sp. $\mathrm{V}_{4} 8$ and (C) Mutant $\Delta_{3463}$ exposed to $10 \%$ cell-free supernatant from Pedobacter sp. $\mathrm{V}_{48} 8$. 


\section{No apparent costs for facultative antibiotic production by the soil bacterium Pseudomonas fluorescens Pfo-1}

\section{Discussion}

Many, if not most, bacteria produce a range of secondary metabolites that can target competing microorganisms (Raaijmakers et al., 2002;Challis and Hopwood, 2003). Natural bacterial populations have been found to consist of a wide variety of genotypes that differ in their ability to both suppress and withstand conspecifics (Ott et al., 2001;Riley et al., 2003;Davelos et al., 2004; Vos and Velicer, 2009). A trade-off between an advantage in growth (resource or scramble competition) and an advantage in 'killing capacity' or 'resistance capacity' (interference competition) lies at the basis of theoretical models attempting to explain the coexistence of strains differing in antibiotic production and sensitivity (Hsu and Waltman, 1997;Czaran and Hoekstra, 2003;Gardner and West, 2004;Czaran and Hoekstra, 2007;Brown et al., 2009; Hibbing et al., 2010). The reason that not all strains evolve to produce antibiotics thus is explained by the fact that the production of these compounds (and their corresponding immunity factors) incurs a metabolic cost. In competition in a structured environment, antibiotic producing cells will displace sensitive (non-antibiotic producing) cells, whereas sensitive (non-antibiotic producing) cells have a growth rate advantage over resistant (non-antibiotic producing) cells that in turn displace antibiotic producing cells because they do not carry the cost of antibiotic production (Kerr et al., 2002).

Previously, it was hypothesized that the facultative- rather than the constitutive production of antibiotics represents a cost-effective strategy, as the antibiotic compound is only produced in situations where it is needed (Garbeva et al., 2011b). Our finding that the cost of antibiotic production in the P. fluorescens Pfo-1 system is not significant is not in line with the costbased assumption on basis of which theoretical models aim to explain how microbial warfare can promote microbial diversity. If these costs are truly insignificant then why do not all strains produce antimicrobial compounds constitutively? Two alternative ecological trade-offs could be envisaged to be at work. First, facultative antibiotic production could prevent competitors evolving resistance to the antibiotic by reducing exposure (Garbeva et al., 2011b). It is evident from clinical studies that increased exposure to antibiotics (through patient consumption) can result in higher resistance levels in a pathogen population (e.g. (Bergman et al., 2004)). Second, it has recently emerged that many bacteria can actually subsist on antibiotic compounds (D'Costa et al., 2006;Dantas et al., 2008). Although antibiotic production 
could inhibit the growth of some strains competing for resources, it could promote the growth of others. It is presently unknown how important both mechanisms are in bacterial populations in soil but they certainly seem worthy of future attention.

Whilst we did not observe significant costs of antibiotic production here, biological costs associated with antibiotic resistance have been reported to vary from significant (e.g. (Andersson, 2003; 2006;Andersson and Hughes, 2010), to no-cost (e.g. (Bjorkman and Andersson, 200o;Kanai et al., 2004; Kugelberg et al., 2005;Zhang et al., 2006)) to even enhanced fitness (e.g. (Zhang et al., 2006)). This variation in costs might be explained by the fact that the genetic systems underlying antibiotic resistance are diverse and furthermore might not be readily comparable to those that underlie antibiotic production (including the production of a molecule conferring autoimmunity). Mutations conferring resistance arising in sensitive cells often modify the molecule targeted by the antibiotic in such a way that, although the cell is protected from the antibiotic, its function is severely compromised (Andersson and Hughes, 2010). Such non-additive, pleiotropic fitness costs often are severe.

With antibiotic production on the other hand, it could be hypothesized that the cells mainly bear the additive, metabolic cost of the production of the antibiotic and the immunity molecules (which must be small compared to the sum of all other molecules produced by the cell) and suffer less from pleiotropic costs. In addition, compensatory mutations that mitigate pleiotropic fitness costs have been identified in resistant bacteria (Andersson and Hughes, 2010). It could be that compensatory mutations are more readily accessible for genetic systems mediating antibiotic production than they are for resistance mutations thus lowering the fitness cost in the former.

Although we did not observe costs for antibiotic production, clear changes in $P$. fluorescens Pfo-1 colony morphology during antibiotic production were apparent from the agar chamber experiments. Antibiotic producing P. fluorescens Pfo-1 cells formed spherical colonies whereas the non-producing mutant as well as wild-type strain in the control situation did form irregular shaped colonies. Recently it was reported that different $P$. fluorescens colony morphology variants have distinct metabolic profiles (Workentine et al., 2010). 
It seems plausible that the spherical colony-shape of Pfo-1 here is a response to the produced antibiotic and not to a signal of Pedobacter, as the mutant deficient in the production of the antibiotics did not produce spherical colonies in the presence of Pedobacter supernatant. Formation of such spherical colonies may coincide with antibiotic production to obtain the highest protection against antagonizing organisms. Further studies are needed to understand the mechanism and the biological relevance of changes in colony morphology of $P$. fluorescens Pfo-1 during antibiotic production.

\section{Acknowledgment}

The authors thank Robin Tecon for his help in designing the agar-incubation chamber and Saskia Gerards for her help in antimicrobial compound extraction. This is publication No.5049 of the Netherlands Institute of Ecology (NIOO-KNAW) 


\section{Supplementary material}

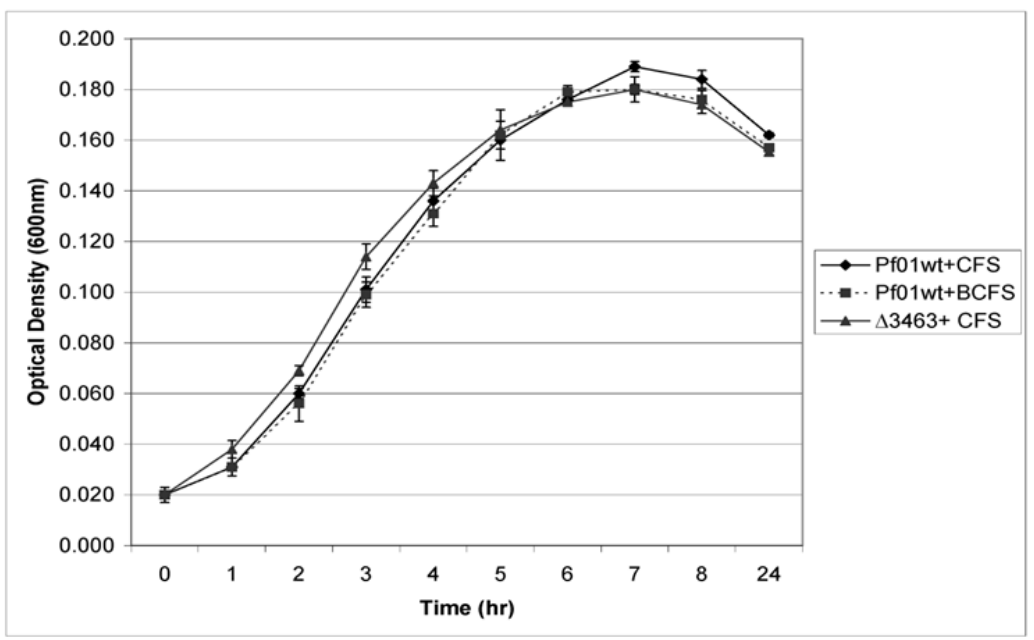

Figure S6.1: Bacterial growth (Optical Density $600 \mathrm{~nm}$ ) in 5oml nutrient-poor liquid cultures: Pforwt+CFS - wild type $P$. fluorescens Pfo-1 with $10 \%$ cell-free supernatant from Pedobacter sp. V48; Pforwt+BCFS - wild type P. fluorescens Pfo-1 with $10 \%$ boiled cell-free supernatant from Pedobacter sp. $\mathrm{V}_{4} 8$; $\Delta 3463+C F S$ - $P$. fluorescens Pfo-1 mutant $\Delta_{3463}$ with $10 \%$ cell-free supernatant from Pedobacter sp. $\mathrm{V}_{4} 8$. The measurements are presented as mean $\pm \mathrm{SD}$ ( $\mathrm{n}=3$ replicates $)$. 


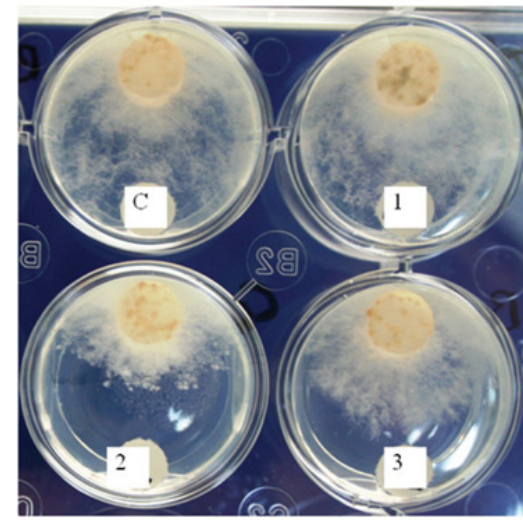

A

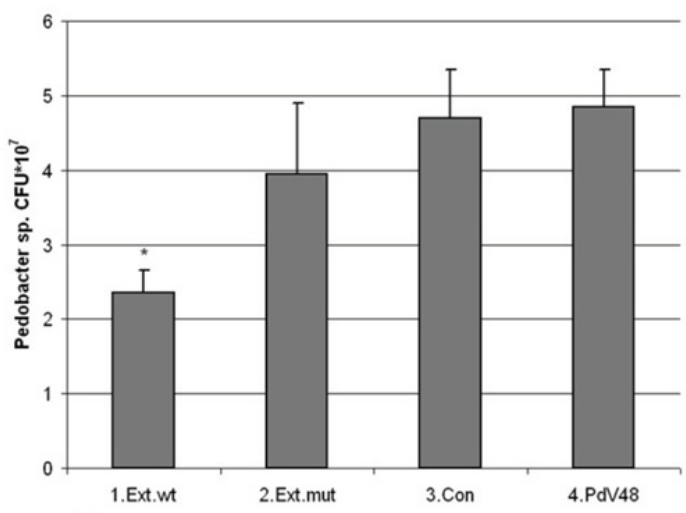

B

Figure S6.2: (A) Antagonist assay against the fungus Rhizoctonia solani with extracts from liquid cultures (Fig 1 SI) 1- Mutant $\Delta_{3463}$ with $10 \%$ cell-free supernatant from Pedobacter sp. V48; 2- wild type P. fluorescens Pfo-1 with $10 \%$ cell-free supernatant from Pedobacter sp. V48; 3- wild type $P$. fluorescens Pfo-1 with $10 \%$ boiled cell-free supernatant from Pedobacter sp. V48 and Ccontrol $50 \%$ methanol. (B) Effect of different extracts from liquid cultures (Figure S6.1) on growth of Pedobacter sp. $\mathrm{V}_{4} 8$ (growing on 1/10 TSBA for 24h) 1.Ext.wt- extract from wild type $P$. fluorescens Pfo-1 with $10 \%$ cell-free supernatant from Pedobacter sp. V48; 2. Ext.mut- extract from mutant $\Delta_{3463}$ with $10 \%$ cell-free supernatant from Pedobacter sp. $\mathrm{V}_{4} 8$ and 3.Con- extract from wild type $P$. fluorescens Pfo-1 with $10 \%$ boiled cell-free supernatant from Pedobacter sp. V48. 4. PdV48- Pedobacter sp. $\mathrm{V}_{4} 8$ without any extract. Data are presented as mean $\pm S D$ ( $n=3$ replicates).

* - Indicates significant reduction of Pedobacter sp. V48 CFU as compared to other treatments $(\mathrm{p}<0.05)$ as analyzed by one-way ANOVA followed by Tukey's HSD test. 
Chapter 7

General discussion 


\section{Impact of interspecific interactions on antimicrobial activity}

What did we know about the influence of interspecific interactions on antimicrobial activity in soil bacteria?

When I started my $\mathrm{PhD}$ project in January 2012 several studies indicated already the importance of interspecific bacterial interactions for triggering of antibiotic production in bacteria (Slattery et al., 2001;de Boer et al., 2007; Garbeva et al., 2011b). Such interaction-mediated induction of antibiotic production was proposed as a promising approach for the discovery of novel antibiotics. For example, co-culturing of two bacteria namely Streptomyces endus S-522 and Tsukamurella pulmonis lead to the discovery of the novel antibiotic compound alchievemycin A (Onaka et al., 2011). However, the frequency of interaction-mediated antibiotic triggering in bacterial communities was unknown and to date most studies on interactions altering antibiotic production are focused mainly on Streptomyces spp. (Ueda et al., 2000;Traxler et al., 2013;Kinkel et al., 2014;Abrudan et al., 2015).

What did my research reveal about the influence of interspecific interactions on antimicrobial activity in soil bacteria?

In chapter two I screened for the first time the influence of interspecific interactions on antimicrobial activity of phylogenetically different bacteria. The results obtained in chapter two indicate clearly that interspecific mediated induction of antibiotic activity is commonly occurring among bacterial species. From all tested bacterial isolates $42 \%$ showed antimicrobial activity only during interactions and $33 \%$ showed antimicrobial activity in monoculture. This frequency is in line with previous studies on frequencies of antimicrobial activity during intra-specific interactions within the genus Streptomyces (Davelos et al., 2004;Kinkel et al., 2014). Yet, the strains that were involved in interaction-mediated triggering of antibiotic production did not produce or induce antibiotic production in all of the tested interactions. This indicates that the identity of the interacting partner is an important factor in the induction of antibiotic production. This is in line with previous observations on a $P$. fluorescens strain for which antibiotic production was found to be dependent on the identity of the interacting species (Garbeva et al., 2011b). The strong influence of the identity of the interacting bacteria could be related to specific signals from neighbouring microorganisms which has been referred to as competitor sensing (Cornforth and Foster, 2013). 
In this thesis bacterial interactions that induced antibiotic production often involved combinations of phylogenetically different bacteria or interactions among beta-Proteobacteria and among Actinobacteria. For the latter phyla several antibiotics have been already identified and described (Pantanella et al., 2007;Berdy, 2012;Cornforth and Foster, 2013;Debois et al., 2013;Zhu, 2014). Hence, it is possible that the screening method revealed bacteria that produce known antibiotics but only during co-cultivation. This would indicate that the regulation is different from known antibiotic-producing bacteria that produce these antibiotics in monoculture.

Some isolates from the phylogenetic classes of Flavobacteria and alphaProteobacteria showed antimicrobial activity only in monocultures, however the majority of these isolates exhibited antimicrobial activity only during interactions. This observation indicates that for these phylogenetic groups there is a clear potential to discover novel antibiotics.

Besides induction of antibiotic production, suppression of antibiotic production was also observed. In total $22 \%$ of all tested interactions suppressed antibiotic production and only $13 \%$ of all tested isolates revealed antimicrobial activity in both monocultures and mixed cultures. Interestingly, this suppressing effect on antibiotic production was more abundant than the induction of antibiotic production. The suppression of antimicrobial activity can be due to several mechanisms e.g. interference with signal transduction systems like the quorum sensing system or other regulation mechanisms involved in antibiotic production (Gonzalez and Keshavan, 2006;Venturi and Subramoni, 2009; Christensen, 2013). Direct growth inhibition of the antibiotic producing strain is also a possible explanation for this observation (Straight et al., 2007;Hibbing et al., 2010;Schneider et al., 2012). Another mechanism for the observed silencing of antibiotic production during interaction could be also the lower nutrient availability, as nutrient supply and nutrient composition play an important role in the production of antimicrobial compounds (van Wezel and McDowall, 2011).

So far it was proposed that the main driving force for antibiotic production is defensive rather than offensive, serving to protect the resources (Chater and Merrick, 1979). Indeed, the results of chapter two show that more bacteria produce antimicrobials during interactions when sensing the presence of a competitor. This is also in line with other studies suggesting that competition 
rather than cooperation dominates interactions of cultivable microbial species (D'Onofrio et al., 2010;Foster and Bell, 2012).

In conclusion, the results obtained in chapter two show that interspecific interactions can significantly affect antimicrobial activity in soil bacteria in both directions: induction and silencing. The high-throughput screening described in chapter two allows for a quick detection of antibiotics produced by bacteria as result of interaction. Such screening of interacting bacteria for antimicrobial activity might be a useful strategy for "waking up" cryptic genes and revealing novel antibiotics and other useful bioactive metabolites.

\section{Impact of interspecific interactions on volatile compound production in bacteria}

What did we know about the impact of bacterial interspecific interactions on volatile production in soil bacteria?

Most studies on microbial volatiles to date are focused on volatiles produced by bacteria cultivated in monocultures (Schulz and Dickschat, 2007;Kai et al., 2009; Wenke et al., 2010;Garbeva et al., 2014a) without considering the effect of bacterial interspecific interactions on volatile compound production in bacteria.

\section{Influence of interspecific interactions on volatile production and volatile blend composition}

In chapter three and four I describe the results of studies on the influence of interspecific interactions on the production of volatile organic compounds in bacteria. I compared the volatile blends emitted by six phylogenetically different soil-bacteria grown either in monoculture or in pair wise cultures.

The results obtained in chapter three and four showed that the volatile blends emitted during interaction differed from the volatile blends of the respective monocultures. In chapter three the volatile blend produced by interacting strains consisted mostly of the volatile compounds produced by the monocultures of those strains. However some compounds that were produced by monocultures were not detected in the mixtures. For example indole was detected in monocultures of Chryseobacterium sp. $\mathrm{AD}_{4} 8$ but was not detected during interaction with Tsukamurella sp. ADı6. Indole and its derivatives are known to play an important role in microbial interactions and 
to be involved in intercellular and multispecies signalling controlling diverse bacterial physiological processes (Wang et al., 2001;Di Martino et al., 2003;Diggle et al., 2006;Nikaido et al., 2008;Lee et al., 2009; Lee and Lee, 2010). In addition to the features as a signal molecule indole has been shown to have inhibitory effects on fungi and stimulating effects of plant growth (Kamath and Vaidyanathan, 1990;Blom et al., 2011). A similar observation was made in chapter five where two volatile compounds that were produced by monocultures of Burkholderia sp. AD24 (S-Methyl methanethiosulfonate and an unknown compound) were not detected during the interaction with Paenibacillus sp. AD87. However, in chapter four induction of volatile compound production during interaction of strains is also reported. One compound identified as 2,5-bis(1-methylethyl)-pyrazine, was produced in higher abundance when Burkholderia sp. AD24 was interacting with Paenibacillus sp. AD87.

\section{Influence of bacterial volatiles on fungal and oomycetal organisms}

Many studies have shown that bacterial volatiles play a major role in the suppression of soil-borne fungi (Zou et al., 2007;Garbeva et al., 2011a;van Agtmaal et al., 2015). In chapter four I also detected strong anti-fungal and anti-oomycetal activity caused by the volatile blends produced by the four tested bacteria cultivated in monocultures or mixed cultures. In line with previous studies I observed strong fungal sensitivity to bacterial volatiles. One possible explanation for the observed strong fungal and oomycetal growth inhibition is the production of sulfur containing volatiles such as dimethyl disulfide, dimethyl trisulfide, which were detected in high abundance. Sulfur containing volatiles like dimethyl di- and trisulfide have been shown to inhibit the growth of different plant pathogenic fungi (Li et al., 2010;Huang et al., 2012;Wang et al., 2013;Kanchiswamy et al., 2015).

\section{Influence of bacterial volatiles on other bacteria}

In chapter three I describe the growth inhibition of $E$. coli WA321 by volatiles emitted by Chryseobacterium sp. $\mathrm{AD}_{4} 8$ and by volatiles emitted by interacting Chryseobacterium and Tsukamurella strains. Besides growth inhibition I also observed growth promotion caused by volatiles, namely the stimulation of growth of $S$. aureus $533 \mathrm{R}_{4}$ by monocultures of Dyella sp. AD56. However, this growth promotion of $S$. aureus was not observed when Dyella sp. AD56 was interacting with Janthinobacterium sp. AD8o. This coincided with an observed shift in volatile blend composition between monocultures and mixed cultures. 
Interestingly the volatiles emitted by the monoculture of Chryseobacterium sp. $\mathrm{AD}_{48} 8$ and the mixture of Dyella sp. $\mathrm{AD}_{5} 6$ with Janthinobacterium sp. AD8o induced clear changes in colony morphology of $S$. marcescens P87. The exposed colonies of $S$. marcescens $\mathrm{P} 87$ appeared to be more spherical as compared to the control. A similar observation was made for $P$. fluorescens Pfo-1 in chapter six, where the micro-colonies also formed round colonies during the exposure to supernatant of a competing bacterium. Hence, it is plausible that this change in bacterial colony morphology is an adaptive response to the produced antimicrobial compounds.

\section{Influence of dimethyl disulfide and dimethyl trisulfide on bacteria}

Most of the volatile compounds detected in the experiments performed in chapter three and four were sulfur-containing volatiles like dimethyl disulfide, dimethyl trisulfide and methyl thiocyanate. Experiments were performed with pure dimethyl di- and trisulfide to test their effect on a variety of bacterial model organisms. The assays revealed strong growth inhibitory capacities on all tested bacterial model organisms, when applied in a concentration of $50 \mu \mathrm{M}$. Such bacterial growth suppression by dimethyl disulfide was already reported for $P$. fluorescens strains against crown-gall diseases causing Agrobacterium sp. (Dandurishvili et al., 2011;Popova et al., 2014). In addition to growth inhibition I also observed influence on colony morphology in S. marcesens P87. Colonies of S. marcesens P87 exposed to dimethyl trisulfide $(50 \mu \mathrm{M})$ showed reduced growth and were lacking the production of prodigiosin. It is possible that this is related to the inhibition of quorum-sensing as previously reported by Chernin in 2011 (Chernin et al., 2011).

Influence of interspecific interactions on the induction of novel volatiles

In chapter four I detected the induction of volatile compound production, namely the production of 2,5-bis(1-methylethyl)-pyrazine with a strong antimicrobial activity. This compound was produced in higher concentration during the interaction of Burkholderia sp. AD24 with Paenibacillus sp. AD87. Hence, the interspecific interactions may lead to the discovery of novel volatile compounds with valuable antimicrobial activities.

In conclusion the results obtained in chapter three and four revealed that interspecific bacterial interactions alter volatile blend composition in both directions induction and suppression. 


\section{Impact of interspecific interactions on bacterial fitness}

What did we know about the impact of bacterial interspecific competitive interactions on bacterial fitness?

Several studies have been done on competitive interactions between bacteria (Hibbing et al., 2010;Foster and Bell, 2012). However there is still little knowledge on competitive interactions between Gram-positive and Gramnegative bacteria.

\section{Effect of interspecific interactions on bacterial fitness}

In two chapters of this thesis the influence of interspecific interactions on bacterial fitness was studied. In chapter four I describe the influence of interspecific interactions on bacterial fitness of Burkholderia sp. AD24 and Paenibacillus sp. AD87 and in chapter five I describe the influence of interspecific interactions on $P$. fluorescens Pfo-1 fitness under two nutrient conditions in sand microcosms.

The results of chapter four showed that the interaction between Burkholderia sp. AD24 and Paenibacillus sp. AD87 had a negative effect on Burkholderia sp. AD24 cell counts whereas the cell counts of Paenibacillus sp. AD87 were not affected. Hence it seems that Paenibacillus sp. A87 is a better competitor than Burkholderia sp. AD24. A similar observation was made in chapter five where the growth of $P$. fluorescens Pfo-1 was negatively affected when confronted with Bacillus sp. V102 resulting in significantly lower cell counts under both nutrient conditions. Although P. fluorescens Pfo-1 cell counts were lower in nutrient poor sand microcosms than in nutrient rich sand microcosms, similar growth patterns were seen under both nutritional conditions. This result indicates that nutrient supply did not have a significant effect on the outcome of the competitive interactions. Yet, it is well known that nutrient conditions can affect the production of secondary metabolites (Sanchez et al., 2010;van Wezel and McDowall, 2011;Garbeva et al., 2014b) and hence can influence the outcome of microbial interactions. Such growth reduction of $P$. fluorescens Pfo-1 in confrontation with Bacillus sp. V102 was already observed on nutrient-poor agar even without direct cell-cell contact (Garbeva and de Boer, 2009). Overall, the results obtained in chapter four and five suggests that the fitness of soil bacteria depends greatly on the presence and identity of the neighboring microorganisms. 


\section{Effect of interspecific interactions on gene expression}

To understand the mechanism of interactions between Gram-positive and Gram-negative bacteria in chapter four I performed transcriptome analysis. The outcome of the transcriptome analysis for Burkholderia sp. AD24 revealed differential expression of ribosomal proteins pointing to a general stress response (Ishige et al., 2003;Silberbach and Burkovski, 2006;Picard et al., 2013). Furthermore several genes related to signal transduction, secondary metabolite production and to cell motility were up-regulated Burkholderia sp. AD24 during the interaction with Paenibacillus sp. AD87. The elevated expression of genes associated with cellular motility (Hu et al., 2009) indicates that motility is important during bacterial interspecific interactions. This observation could imply that Burkholderia sp. $\mathrm{AD}_{24}$ is trying to escape from Paenibacillus sp. AD87. Such elevated expression of genes related to cell motility during interspecific interaction between Gram-negative and Grampositive bacteria was previously observed for P. fluorescens Pfo-1 (Garbeva et al., 2011b).

For Paenibacillus sp. AD87, genes encoding antibiotic resistance were highly up regulated and in particular genes related to the Vancomycin B-type resistance, suggesting protection against antimicrobial compounds produced by Burkholderia sp. AD24. Recently, Letoffe and co-workers (Letoffe et al., 2014) reported the increase of antibiotic resistance due to possible synergistic effects between volatile compounds, thus it is possible that in this particular case, the produced volatile compounds such as 2,5-bis(1-methylethyl)pyrazine induced increased expression of genes related to antibiotic resistance in Paenibacillus sp. AD87.

\section{Effect of interspecific interactions on secondary metabolite production} The performed metabolomic analysis in chapter four revealed that the produced metabolites by monocultures and interactions of Burkholderia sp. $\mathrm{AD}_{24}$ and Paenibacillus sp. AD87 differed. The interaction increased the production of specific antimicrobial compounds such as 2,5-bis(1methylethyl)-pyrazine and as well of a unknown soluble pederin like compound $\left(\mathrm{C}_{25} \mathrm{H}_{45} \mathrm{NO} 9, \mathrm{~m} / \mathrm{z}=504.316\right)\left[\mathrm{M}^{+} \mathrm{H}^{+}\right]$. The production of 2,5 -bis $\left(1^{-}\right.$ methylethyl)-pyrazine was so far only reported for a few bacteria (Beck et al., 2003;Dickschat et al., 2005;Rajini et al., 2011). 
In chapter four strong synergistic effects between 2,5-bis(1-methylethyl)pyrazine and the soluble secondary metabolites produced during interaction of Burkholderia sp. AD24 with Paenibacillus sp. AD87 was observed. This stronger antimicrobial activity might be related to synergistic effects between non-volatile and volatile compounds enhancing the overall antimicrobial activity (Schmidt et al., 2015). Such synergistic effects between volatile and non-volatile compounds were already reported for other antibiotics like e.g. beta-lactam antibiotics (Hemaiswarya and Doble, 2010).

In conclusion from the results described in chapter four and five it is obvious that interspecific bacterial interactions are important and influence microbial fitness, gene expression and the production of secondary (volatile and soluble) metabolites and consequently affect the structure of microbial communities.

\section{Biological costs for the production of antimicrobial compounds}

What did we knew about the possible costs of antimicrobial compound production in bacteria?

The knowledge about the possible costs for the production of antimicrobial compound by bacteria is scarce. While there are several studies showing the possible biological costs of antibiotic resistance (Gagneux et al., 2006;Andersson and Hughes, 2010;Melnyk et al., 2015) so far there were no studies showing the costs for antimicrobial compound production in bacteria. Yet, it is generally assumed that the production of antimicrobial compounds and their corresponding immunity factors incurs metabolic costs for the producing organism.

In chapter six I studied the possible costs for facultative antimicrobial compound production in $P$. fluorescens Pfo-1 by monitoring microscopically the growth rate of micro-colonies with and without induction of antibiotic production. The results obtained in this chapter showed that the production of antimicrobial compounds does not incur significant costs for $P$. fluorescens Pfo-1. An alternative explanation for reduced costs of antibiotic production is that such competition-mediated induction of antimicrobial compound production lowers the risk of increasing resistance of competitors against the produced antibiotic compound as well it lowers the risk of competing organisms to feed on the produced antimicrobial compound. 
In conclusion the results revealed in chapter six showed that production of antimicrobial compounds is not necessarily costly for the antibiotic compound produced by $P$. fluorescens Pfo-1 as no reduction of growth was observed. A similar observation was made in chapter five were $P$. fluorescens Pfo-1 growing in microcosms together with Pedobacter sp. V48 was triggered for the productions of antibiotics but didn't showed reduced growth. If this observation is generally valid for antibiotic production by soil bacteria then it will have consequences for the prediction of producer /non-producer (cheaters) dynamics in bacterial populations.

\section{Outlook on the discovery of novel antibiotics and general conclusion}

There is a need for new antimicrobial drugs with activity against both pathogenic bacteria and fungi. In the last four decades antibiotic resistance in pathogenic bacteria has become a global rising health problem (Crisóstomo et al., 2001;Tenover et al., 2001;Al-Gheethi et al., 2013;Economou et al., 2013). However, so far, only two classes of new antibiotics have reached the clinical practice, so there is a clear discrepancy between the number of newly discovered antibiotics and the number of novel compounds which would be needed to fight the problem of antimicrobial resistance (Barbachyn and Ford, 2003;Kern, 2006;ECDC/EMEA, 2009). Antimicrobial resistance is a complex problem that requires efforts of microbiologists, ecologists, health care specialists, educationalists, the industry, policy makers and the public in order to be solved.

This $\mathrm{PhD}$ project was financed by the BE- Basic consortium with a practical background aiming for the discovery of novel antimicrobial compounds by using a so called "intelligent mining" approach by screening interacting bacteria for novel antimicrobial activity. The high-throughput screening method developed in this work allows a fast screening of interacting bacteria for antimicrobial activity.

Mining into bacterial interspecific interactions is one way of "waking up" cryptic gene clusters in order to reveal novel and potentially useful secondary metabolites. Furthermore studying bacterial interspecific interactions that affect antimicrobial activity may be very beneficial to better understand the composition and the dynamics of soil microbial communities (Velicer, 2003; Mitri and Foster, 2013;Abrudan et al., 2015;Aziz et al., 2015). Further understanding of such bacterial interspecific interactions can be additionally beneficial for selecting the right players in synthetic communities that fulfil 188 
specific ecosystem services like disease suppression in agricultural crop systems (Weller et al., 2002;Garbeva et al., 2004;De Roy et al., 2013;Grosskopf and Soyer, 2014).

So far most of the studies on secondary metabolites produced by bacteria are focused either on soluble or on volatile organic compounds alone but do not consider the importance and the possible synergistic effects of both compound classes together as shown in this thesis.

In this thesis we show that volatile compounds can exert synergistic effects with soluble compounds and enhance the overall antimicrobial activity. However, very often the application of bacteria to control soil-borne diseases fails, as the diseases suppression is not consistent or minimal. Thus, selection of a mixture of interacting strains with different properties may lead to more consistent disease control in agricultural production systems.

Overall this work contributes to better understanding of microbial interactions and in particular the interaction between soil bacteria. However, interactions in nature are much more complex and can vary depending on the occurring environmental conditions. Thus laboratory experiments are only a snapshot of the "real" situation and will not give a complete picture of the complex interactions occurring in nature.

To finalize, the results described in this thesis show that the screening of interspecific bacterial interactions can be important (1) to understand competitive mechanisms occurring in soil bacterial communities and (2) for the discovery of novel volatile and soluble antimicrobial compounds important for agriculture as well for medical purposes. 
General discussion 


\section{References}




\section{References}

Abrudan, M.I., Smakman, F., Grimbergen, A.J., Westhoff, S., Miller, E.L., Van Wezel, G.P., and Rozen, D.E. (2015). Socially mediated induction and suppression of antibiosis during bacterial coexistence. Proceedings of the National Academy of Sciences 112, 11054-11059. doi: 10.1073/pnas.1504076112.

Al-Gheethi, A.a.S., Ismail, N., Lalung, J., Talib, A., Efaq, A.N., and Ab Kadir, M.O. (2013). Susceptibility for antibiotics among faecal indicators and pathogenic bacteria in sewage treated effluents. Water Practice and Technology 8, 1-6.

Alden, L., Demoling, F., and Baath, E. (2001). Rapid method of determining factors limiting bacterial growth in soil. Applied and Environmental Microbiology 67, 1830-1838.

Allen, B., and Nowak, M.A. (2013). Cooperation and the fate of microbial societies. PLoS Biol 11, e1001549. doi: 10.1371/journal.pbio.1001549.

Altschul, S.F., Madden, T.L., Schaffer, A.A., Zhang, J.H., Zhang, Z., Miller, W., and Lipman, D.J. (1997). Gapped BLAST and PSI-BLAST: a new generation of protein database search programs. Nucleic Acids Research 25, 3389-3402. doi: DOI 10.1093/nar/25.17.3389.

Anand, R., Grayston, S., and Chanway, C. (2013). N2-Fixation and Seedling Growth Promotion of Lodgepole Pine by Endophytic Paenibacillus polymyxa. Microbial Ecology 66, 369-374. doi: 10.1007/soo248-013o196-1.

Anderl, J.N., Zahller, J., Roe, F., and Stewart, P.S. (2003). Role of nutrient limitation and stationary-phase existence in Klebsiella pneumoniae biofilm resistance to ampicillin and ciprofloxacin. Antimicrob Agents Chemother 47, 1251-1256.

Andersson, D.I. (2003). Persistence of antibiotic resistant bacteria. Current Opinion in Microbiology 6, 452-456. doi: 10.1016/j.mib.2003.09.001.

Andersson, D.I. (2006). The biological cost of mutational antibiotic resistance: any practical conclusions? Current Opinion in Microbiology 9, 461465.

Andersson, D.I., and Hughes, D. (2010). Antibiotic resistance and its cost: is it possible to reverse resistance? Nature Reviews Microbiology 8, 260271. doi: 10.1038/nrmicro2319.

Audrain, B., Farag, M.A., Ryu, C.M., and Ghigo, J.M. (2015). Role of bacterial volatile compounds in bacterial biology. Fems Microbiology Reviews 39, 222-233. doi: 10.1093/femsre/fuuo13.

Aziz, F.a.A., Suzuki, K., Ohtaki, A., Sagegami, K., Hirai, H., Seno, J., Mizuno, N., Inuzuka, Y., Saito, Y., Tashiro, Y., Hiraishi, A., and Futamata, H. (2015). Interspecies interactions are an integral determinant of microbial community dynamics. Frontiers in Microbiology 6. doi: 10.3389/fmicb.2015.01148. 
Baba, T., Ara, T., Hasegawa, M., Takai, Y., Okumura, Y., Baba, M., Datsenko, K.A., Tomita, M., Wanner, B.L., and Mori, H. (2006). Construction of Escherichia coli K-12 in-frame, single-gene knockout mutants: the Keio collection. Molecular Systems Biology 2, 2006 ooo8. doi: 10.1038/msb4100050.

Bais, H.P., Weir, T.L., Perry, L.G., Gilroy, S., and Vivanco, J.M. (2006). The role of root exudates in rhizosphere interactions with plants and other organisms. Annu Rev Plant Biol 57, 233-266. doi: 10.1146/annurev.arplant.57.032905.105159.

Balouiri, M., Sadiki, M., and Ibnsouda, S.K. (2016). Methods for in vitro evaluating antimicrobial activity: A review. Journal of Pharmaceutical Analysis 6, 71-79. doi: http://dx.doi.org/10.1016/j.jpha.2015.11.005.

Baltz, R.H. (2008). Renaissance in antibacterial discovery from actinomycetes. Current Opinion Pharmacology 8, 557-563. doi: 10.1016/j.coph.2008.04.008.

Barbachyn, M.R., and Ford, C.W. (2003). Oxazolidinone Structure-Activity Relationships Leading to Linezolid. Angewandte Chemie International Edition 42, 2010-2023. doi: 10.1002/anie.200200528.

Beck, H.C., Hansen, A.M., and Lauritsen, F.R. (2003). Novel pyrazine metabolites found in polymyxin biosynthesis by Paenibacillus polymyxa. Fems Microbiology Letters 220, 67-73.

Becker, J., Eisenhauer, N., Scheu, S., and Jousset, A. (2012). Increasing antagonistic interactions cause bacterial communities to collapse at high diversity. Ecology Letters 15, 468-474. doi: 10.1111/j.14610248.2012.01759.x.

Berdy, J. (2012). Thoughts and facts about antibiotics: Where we are now and where we are heading. Journal of Antibiotics 65, 385-395. doi: Doi 10.1038/Ja.2012.27.

Berg, G., Grube, M., Schloter, M., and Smalla, K. (2014). The plant microbiome and its importance for plant and human health. Front Microbiol 5, 491. doi: 10.3389/fmicb.2014.00491.

Berge, O., Guinebretière, M.-H., Achouak, W., Normand, P., and Heulin, T. (2002). Paenibacillus graminis sp. nov. and Paenibacillus odorifer sp. nov., isolated from plant roots, soil and food. International Journal of Systematic and Evolutionary Microbiology 52, 607-616. doi: doi:10.1099/00207713-52-2-607.

Bergman, M., Huikko, S., Pihlajamaki, M., Laippala, P., Palva, E., Huovinen, P., Seppala, H., and Finnish Study Grp Antimicrobial, R. (2004). Effect of macrolide consumption on erythromycin resistance in Streptococcus pyogenes in Finland in 1997-2001. Clinical Infectious Diseases 38, 1251-1256.

Bertrand, S., Bohni, N., Schnee, S., Schumpp, O., Gindro, K., and Wolfender, J.-L. (2014). Metabolite induction via microorganism co-culture: A potential way to enhance chemical diversity for drug discovery. 
Biotechnology Advances 32, 1180-1204. doi: http://dx.doi.org/10.1016/j.biotechadv.2014.03.001.

Beshkova, D.M., Simova, E.D., Frengova, G.I., Simov, Z.I., and Dimitrov, Z.P. (2003). Production of volatile aroma compounds by kefir starter cultures. International Dairy Journal 13, 529-535. doi: Doi 10.1016/So958-6946(o3)ooo58-X.

Binet, R., and Maurelli, A.T. (2005). Fitness cost due to mutations in the $16 \mathrm{~S}$ rRNA associated with spectinomycin resistance in Chlamydia psittaci 6BC. Antimicrobial Agents and Chemotherapy 49, 4455-4464. doi: 10.1128/aac.49.11.4455-4464.2005.

Bjarnason, J., Southward, C.M., and Surette, M.G. (2003). Genomic profiling of iron-responsive genes in Salmonella enterica serovar typhimurium by high-throughput screening of a random promoter library. J Bacteriol 185, 4973-4982.

Bjorkman, J., and Andersson, D.I. (2000). The cost of antibiotic resistance from a bacterial perspective. Drug Resistance Updates 3, 237-245.

Bjorkman, J., Hughes, D., and Andersson, D.I. (1998). Virulence of antibioticresistant Salmonella typhimurium. Proceedings of the National Academy of Sciences 95, 3949-3953.

Bjurman, J. (2007). "Release of MVOCs from Microorganisms," in Organic Indoor Air Pollutants. Wiley-VCH Verlag GmbH), 259-273.

Blom, D., Fabbri, C., Connor, E.C., Schiestl, F.P., Klauser, D.R., Boller, T., Eberl, L., and Weisskopf, L. (2011). Production of plant growth modulating volatiles is widespread among rhizosphere bacteria and strongly depends on culture conditions. Environmental Microbiology 13, 3047-3058. doi: 10.1111/j.1462-2920.2011.02582.x.

Bonfante, P., and Anca, I.A. (2009). Plants, mycorrhizal fungi, and bacteria: a network of interactions. Annual Review of Microbiology 63, 363-383. doi: 10.1146/annurev.micro.091208.073504.

Bonkowski, M., Villenave, C., and Griffiths, B. (2009). Rhizosphere fauna: the functional and structural diversity of intimate interactions of soil fauna with plant roots. Plant and Soil 321, 213-233. doi: 10.1007/s11104009-0013-2.

Bosshard, P.P., Zbinden, R., and Altwegg, M. (2002). Paenibacillus turicensis sp. nov., a novel bacterium harbouring heterogeneities between $16 \mathrm{~S}$ rRNA genes. International Journal of Systematic and Evolutionary Microbiology 52, 2241-2249. doi: doi:10.1099/00207713-52-6-2241.

Braatsch, S., Helmark, S., Kranz, H., Koebmann, B., and Jensen, P.R. (2008). Escherichia coli strains with promoter libraries constructed by Red/ET recombination pave the way for transcriptional fine-tuning. Biotechniques 45, 335-337. doi: 10.2144/o00112907.

Brown, S.P., Inglis, R.F., and Taddei, F. (2009). Evolutionary ecology of microbial wars: within-host competition and (incidental) virulence. 
Evolutionary Applications 2, $32-39$. doi: $10.1111 / \mathrm{j} .1752-$ 4571.2008.00059.x.

Buée, M., Boer, W., Martin, F., Overbeek, L., and Jurkevitch, E. (2009). The rhizosphere zoo: An overview of plant-associated communities of microorganisms, including phages, bacteria, archaea, and fungi, and of some of their structuring factors. Plant and Soil 321, 189-212. doi: 10.1007/s11104-009-9991-3.

Carter, G.T. (2014). NP/MS since 1970: from the basement to the bench top. Natural Product Reports 31, 711-717. doi: 10.1039/c3np70085b.

Challis, G.L., and Hopwood, D.A. (2003). Synergy and contingency as driving forces for the evolution of multiple secondary metabolite production by Streptomyces species. Proceedings of the National Academy of Sciences of the United States of America 100, 14555-14561. doi: DOI 10.1073/pnas.1934677100.

Chater, K., and Merrick, M. (1979). "Developmental biology of prokaryotes," ed. P. Jh. Blackwell Publishing, Oxford, United Kingdom), 93 - 114.

Chernin, L., Toklikishvili, N., Ovadis, M., Kim, S., Ben-Ari, J., Khmel, I., and Vainstein, A. (2011). Quorum-sensing quenching by rhizobacterial volatiles. Environ Microbiol Rep 3, 698-704. doi: 10.1111/j.17582229.2011.00284.X.

Chiang, Y.M., Chang, S.L., Oakley, B.R., and Wang, C.C.C. (2011). Recent advances in awakening silent biosynthetic gene clusters and linking orphan clusters to natural products in microorganisms. Current Opinion in Chemical Biology 15, 137-143. doi: DOI 10.1016/j.cbpa.2010.10.011.

Christensen, L.D., M. Van Gennip, Et Al. (2013). Clearance of Pseudomonas aeruginosa Foreign-Body Biofilm Infections through Reduction of the Cyclic Di-GMP Level in Bacteria. Infection and Immunity 81, 27052731.

Cleason, A. (2006). Volatile organic compounds from microorganisms Ph.D Thesis, UMEÅ UNIVERSITY.

Cochrane, S.A., and Vederas, J.C. (2016). Lipopeptides from Bacillus and Paenibacillus spp.: A Gold Mine of Antibiotic Candidates. Medicinal Research Reviews 36, 4-31. doi: 10.1002/med.21321.

Coenye, T., and Vandamme, P. (2003). Diversity and significance of Burkholderia species occupying diverse ecological niches. Environmental Microbiology 5, 719-729. doi: 10.1046/j.14622920.2003.00471.X.

Compant, S., Nowak, J., Coenye, T., Clément, C., and Ait Barka, E. (2008). Diversity and occurrence of Burkholderia spp. in the natural environment. Fems Microbiology Reviews 32, 607-626. doi: 10.1111/j.1574-6976.2008.00113.x.

Compant, S., Reiter, B., Sessitsch, A., Nowak, J., Clément, C., and Ait Barka, E. (2005). Endophytic Colonization of Vitis vinifera L. by Plant Growth- 
Promoting Bacterium Burkholderia sp. Strain PsJN. Applied and Environmental Microbiology 71, 1685-1693. doi: 10.1128/aem.71.4.16851693.2005.

Compeau, G., Alachi, B.J., Platsouka, E., and Levy, S.B. (1988). Survival of rifampin-resistant mutants of Pseudomonas fluorescens and Pseudomonas putida in soil systems. Applied and Environmental Microbiology 54, 2432-2438.

Cornforth, D.M., and Foster, K.R. (2013). Competition sensing: the social side of bacterial stress responses. Nature Reviews Microbiology 11, 285-293. doi: Doi 10.1038/Nrmicro2977.

Coustau, C., Chevillon, C., and Ffrench-Constant, R. (2000). Resistance to xenobiotics and parasites: can we count the cost? Trends in Ecology $\mathcal{E}$ Evolution 15, 378-383.

Crespi, B.J. (2001). The evolution of social behavior in microorganisms. Trends in Ecology \& Evolution 16, 178-183. doi: http://dx.doi.org/10.1016/So169-5347(01)02115-2.

Crisóstomo, M.I., Westh, H., Tomasz, A., Chung, M., Oliveira, D.C., and De Lencastre, H. (2001). The evolution of methicillin resistance in Staphylococcus aureus: Similarity of genetic backgrounds in historically early methicillin-susceptible and -resistant isolates and contemporary epidemic clones. Proc Natl Acad Sci U S A 98, 98659870.

Curtis, T.P., Sloan, W.T., and Scannell, J.W. (2002). Estimating prokaryotic diversity and its limits. Proc Natl Acad Sci U S A 99, 10494-10499. doi: 10.1073/pnas.142680199.

Czaran, T., and Hoekstra, R.F. (2007). A spatial model of the evolution of quorum sensing regulating bacteriocin production. Behavioral Ecology 18, 866-873. doi: 10.1093/beheco/armo61.

Czaran, T., and Hoekstra, R.F. (2009). Microbial communication, cooperation and cheating: quorum sensing drives the evolution of cooperation in bacteria. Plos One 4, e6655. doi: 10.1371/journal.pone.0006655.

Czaran, T.L., and Hoekstra, R.F. (2003). Killer-sensitive coexistence in metapopulations of micro-organisms. Proceedings of the Royal Society B-Biological Sciences 270, 1373-1378. doi: 10.1098/rspb.2003.2338.

D'costa, V.M., Mcgrann, K.M., Hughes, D.W., and Wright, G.D. (2006). Sampling the antibiotic resistome. Science 311, 374-377. doi: 10.1126/science.112080o.

D'onofrio, A., Crawford, J.M., Stewart, E.J., Witt, K., Gavrish, E., Epstein, S., Clardy, J., and Lewis, K. (2010). Siderophores from neighboring organisms promote the growth of uncultured bacteria. Chemistry $\mathcal{E}$ Biology 17, 254-264. doi: 10.1016/j.chembiol.2010.02.010.

Da Mota, F.F., Gomes, E.A., Paiva, E., and Seldin, L. (2005). Assessment of the diversity of Paenibacillus species in environmental samples by a novel 
rpoB-based PCR-DGGE method. Fems Microbiology Ecology 53, 317328. doi: 10.1016/j.femsec.2005.01.017.

Daane, L.L., Harjono, I., Barns, S.M., Launen, L.A., Palleron, N.J., and Häggblom, M.M. (2002). PAH-degradation by Paenibacillus spp. and description of Paenibacillus naphthalenovorans sp. nov., a naphthalene-degrading bacterium from the rhizosphere of salt marsh plants. International Journal of Systematic and Evolutionary Microbiology 52, 131-139. doi: doi:10.1099/00207713-52-1-131.

Dandurishvili, N., Toklikishvili, N., Ovadis, M., Eliashvili, P., Giorgobiani, N., Keshelava, R., Tediashvili, M., Vainstein, A., Khmel, I., Szegedi, E., and Chernin, L. (2011). Broad-range antagonistic rhizobacteria Pseudomonas fluorescens and Serratia plymuthica suppress Agrobacterium crown gall tumours on tomato plants. Journal of Applied Microbiology 110, 341-352. doi: 10.1111/j.1365-2672.2010.04891.x.

Dantas, G., Sommer, M.O.A., Oluwasegun, R.D., and Church, G.M. (2008). Bacteria subsisting on antibiotics. Science 320, 100-103. doi: 10.1126/science.1155157.

Davelos, A.L., Kinkel, L.L., and Samac, D.A. (2004). Spatial variation in frequency and intensity of antibiotic interactions among Streptomycetes from prairie soil. Applied and Environmental Microbiology 70, 1051-1058.

De Boer, W., Kowalchuk, G.A., and Van Veen, J.A. (2006). 'Root-food' and the rhizosphere microbial community composition. New Phytologist 170, 3-6. doi: 10.1111/j.1469-8137.2006.01674.x.

De Boer, W., Wagenaar, A.M., Klein Gunnewiek, P.J., and Van Veen, J.A. (2007). In vitro suppression of fungi caused by combinations of apparently non-antagonistic soil bacteria. FEMS Microbiology Ecology 59, 177-185. doi: 10.1111/j.1574-6941.2006.00197.x.

De Carvalho, K.G., Bambirra, F.H., Kruger, M.F., Barbosa, M.S., Oliveira, J.S., Santos, A.M., Nicoli, J.R., Bemquerer, M.P., De Miranda, A., Salvucci, E.J., Sesma, F.J., and Franco, B.D. (2010). Antimicrobial compounds produced by Lactobacillus sakei subsp. sakei $2 \mathrm{a}$, a bacteriocinogenic strain isolated from a Brazilian meat product. J Ind Microbiol Biotechnol 37, 381-39o. doi: 10.1007/s10295-009-0684-y.

De Ridder-Duine, A.S., Kowalchuk, G.A., Klein Gunnewiek, P.J.A., Smant, W., Van Veen, J.A., and De Boer, W. (2005). Rhizosphere bacterial community composition in natural stands of Carex arenaria (sand sedge) is determined by bulk soil community composition. Soil Biology \& Biochemistry 37, 349-357. doi: DOI 10.1016/j.soilbio.2004.08.005.

De Roy, K., Marzorati, M., Van Den Abbeele, P., Van De Wiele, T., and Boon, N. (2013). Synthetic microbial ecosystems: an exciting tool to understand and apply microbial communities. Environmental Microbiology. doi: 10.1111/1462-2920.12343. 
Debois, D., Ongena, M., Cawoy, H., and De Pauw, E. (2013). MALDI-FTICR MS Imaging as a Powerful Tool to Identify Paenibacillus Antibiotics Involved in the Inhibition of Plant Pathogens. Journal of the American Society for Mass Spectrometry 24, 1202-1213. doi: DOI 10.1007/s13361013-0620-2.

Deetae, P., Mounier, J., Bonnarme, P., Spinnler, H.E., Irlinger, F., and Helinck, S. (2009). Effects of Proteus vulgaris growth on the establishment of a cheese microbial community and on the production of volatile aroma compounds in a model cheese. Journal of Applied Microbiology 107, 1404-1413. doi: DOI 10.1111/j.1365-2672.2009.04315.x.

Demoling, F., Figueroa, D., and Baath, E. (2007). Comparison of factors limiting bacterial growth in different soils. Soil Biology \& Biochemistry 39, 2485-2495. doi: DOI 10.1016/j.soilbio.2007.05.002.

Dennis, P.G., Miller, A.J., and Hirsch, P.R. (2010). Are root exudates more important than other sources of rhizodeposits in structuring rhizosphere bacterial communities? Fems Microbiology Ecology 72, 313-327. doi: 10.1111/j.1574-6941.2010.00860.x.

Di Martino, P., Fursy, R., Bret, L., Sundararaju, B., and Phillips, R.S. (2003). Indole can act as an extracellular signal to regulate biofilm formation of Escherichia coli and other indole-producing bacteria. Canadian Journal of Microbiology 49, 443-449.

Dickschat, J.S., Reichenbach, H., Wagner-Dobler, I., and Schulz, S. (2005). Novel pyrazines from the myxobacterium Chondromyces crocatus and marine bacteria. European Journal of Organic Chemistry, 41414153. doi: DOI 10.1002/ejoc.20050028o.

Diggle, S.P., Cornelis, P., Williams, P., and Cámara, M. (2006). 4-Quinolone signalling in Pseudomonas aeruginosa: Old molecules, new perspectives. International Journal of Medical Microbiology 296, 83-91. doi: http://dx.doi.org/10.1016/j.ijmm.2006.01.038.

Ecdc/Emea (2009). The bacterial challenge: time to react A call to narrow the gap between multidrug-resistant bacteria in the $\mathrm{EU}$ and the development of new antibacterial agents. ECDC/EMEA Joint technical report.

Economou, V., Gousia, P., Kansouzidou, A., Sakkas, H., Karanis, P., and Papadopoulou, C. (2013). Prevalence, antimicrobial resistance and relation to indicator and pathogenic microorganisms of Salmonella enterica isolated from surface waters within an agricultural landscape. International Journal of Hygiene and Environmental Health 216, 435444. doi: DOI 10.1016/j.ijheh.2012.07.004.

Edwards, U., Rogall, T., Blocker, H., Emde, M., and Bottger, E.C. (1989). Isolation and Direct Complete Nucleotide Determination of Entire Genes - Characterization of a Gene Coding for 16s-Ribosomal Rna. Nucleic Acids Research 17, 7843-7853. doi: DOI 10.1093/nar/17.19.7843. 
Effmert, U., Kalderas, J., Warnke, R., and Piechulla, B. (2012). Volatile mediated interactions between bacteria and fungi in the soil. $J$ Chem Ecol 38, 665-703. doi: 10.1007/s10886-012-0135-5.

Ender, M., Mccallum, N., Adhikari, R., and Berger-Bachi, B. (2004). Fitness cost of SCCmec and methicillin resistance levels in staphylococcus aureus. Antimicrobial Agents and Chemotherapy 48, 2295-2297. doi: 10.1128/aac.48.6.2295-2297.2004.

Engledow, A.S., Medrano, E.G., Mahenthiralingam, E., Lipuma, J.J., and Gonzalez, C.F. (2004). Involvement of a plasmid-encoded type IV secretion system in the plant tissue watersoaking phenotype of Burkholderia cenocepacia. J Bacteriol 186, 6015-6024. doi: 10.1128/JB.186.18.6015-6024.2004.

Evers, S., and Courvalin, P. (1996). Regulation of VanB-type vancomycin resistance gene expression by the $\operatorname{VanS}(\mathrm{B})$-VanR (B) two-component regulatory system in Enterococcus faecalis V583. Journal of Bacteriology 178, 1302-1309.

Farag, M.A., Porzel, A., and Wessjohann, L.A. (2012). Comparative metabolite profiling and fingerprinting of medicinal licorice roots using a multiplex approach of GC-MS, LC-MS and $1 \mathrm{D}$ NMR techniques. Phytochemistry 76, 60-72. doi: 10.1016/j.phytochem.2011.12.010.

Fiddaman, P.J., and Rossall, S. (1993). The production of antifungal volatiles by Bacillus subtilis. Journal of Applied Bacteriology 74, 119-126.

Fiegna, F., and Velicer, G.J. (2005). Exploitative and Hierarchical Antagonism in a Cooperative Bacterium. PLoS Biol 3, e37o. doi: 10.1371/journal.pbio.0030370.

Fierer, N., Jackson, J.A., Vilgalys, R., and Jackson, R.B. (2005). Assessment of soil microbial community structure by use of taxon-specific quantitative PCR assays. Appl Environ Microbiol 71, 4117-4120. doi: 10.1128/AEM.71.7.4117-4120.2005.

Firn, R.D., Jones, C. G (2003). Natural products - a simle model to explain chemical diversity. Natural Product Reports 20, 382-391.

Fitter, A.H., Gilligan, C.A., Hollingworth, K., Kleczkowski, A., Twyman, R.M., Pitchford, J.W., and The Members of the Nerc Soil Biodiversity, P. (2005). Biodiversity and ecosystem function in soil. Functional Ecology 19, 369-377. doi: 10.1111/j.0269-8463.2005.00969.x.

Foster, Kevin r., and Bell, T. (2012). Competition, not cooperation, dominates interactions among culturable microbial species. Current biology 22, 1845-1850. doi: http://dx.doi.org/10.1016/i.cub.2012.08.005.

Frank, J.H., and Kanamitsu, K. (1987). Paederus, Sensu Lato (Coleoptera: Staphylinidae): Natural History and Medical Importance. Journal of Medical Entomology 24, 155-191. doi: 10.1093/jmedent/24.2.155.

Gagneux, S., Long, C.D., Small, P.M., Van, T., Schoolnik, G.K., and Bohannan, B.J.M. (2006). The competitive cost of antibiotic resistance in 
Mycobacterium tuberculosis. Science 312, 1944-1946. doi: 10.1126/science.1124410.

Gallucci, M.N., Oliva, M., Casero, C., Dambolena, J., Luna, A., Zygadlo, J., and Demo, M. (2009). Antimicrobial combined action of terpenes against the food-borne microorganisms Escherichia coli, Staphylococcus aureus and Bacillus cereus. Flavour and Fragrance Journal 24, 348-354. doi: $10.1002 / \mathrm{ffj} .1948$.

Galperin, M.Y., and Koonin, E.V. (2004). 'Conserved hypothetical' proteins: prioritization of targets for experimental study. Nucleic Acids Research 32, 5452-5463. doi: 10.1093/nar/gkh885.

Galperin, M.Y., Makarova, K.S., Wolf, Y.I., and Koonin, E.V. (2015). Expanded microbial genome coverage and improved protein family annotation in the COG database. Nucleic Acids Research 43, D261-269. doi: 10.1093/nar/gku1223.

Gans, J., Wolinsky, M., and Dunbar, J. (2005). Computational improvements reveal great bacterial diversity and high metal toxicity in soil. Science 309, 1387-139o. doi: DOI 10.1126/science.1112665.

Garbeva, P., and De Boer, W. (2009). Inter-specific Interactions Between Carbon-limited Soil Bacteria Affect Behavior and Gene Expression. Microbial Ecology 58, 36-46. doi: 10.1007/soo248-009-9502-3.

Garbeva, P., Hol, W.H.G., Termorshuizen, A.J., Kowalchuk, G.A., and De Boer, W. (2011a). Fungistasis and general soil biostasis - A new synthesis. Soil Biology \& Biochemistry 43, 469-477. doi: DOI 10.1016/j.soilbio.2010.11.020.

Garbeva, P., Hordijk, C., Gerards, S., and De Boer, W. (2014a). Volatilemediated interactions between phylogenetically different soil bacteria. Front Microbiol 5, 289. doi: 10.3389/fmicb.2014.00289.

Garbeva, P., Hordijk, C., Gerards, S., and De Boer, W. (2014b). Volatiles produced by the mycophagous soil bacterium Collimonas. Fems Microbiology Ecology 87, 639-649. doi: 10.1111/1574-6941.12252.

Garbeva, P., Silby, M.W., Raaijmakers, J.M., Levy, S.B., and De Boer, W. (2011b). Transcriptional and antagonistic responses of Pseudomonas fluorescens Pfo-1 to phylogenetically different bacterial competitors. ISME Journal 5, 973-985.

Garbeva, P., Tyc, O., Remus-Emsermann, M.N.P., Van Der Wal, A., Vos, M., Silby, M., and De Boer, W. (2011c). No Apparent Costs for Facultative Antibiotic Production by the Soil Bacterium Pseudomonas fluorescens Pfo-1. Plos One 6. doi: 10.1371/journal.pone.0027266.

Garbeva, P., Van Veen, J.A., and Van Elsas, J.D. (2003). Predominant Bacillus spp. in agricultural soil under different management regimes detected via PCR-DGGE. Microbial Ecology 45, 302-316. doi: DOI 10.1007/s00248-002-2034-8.

Garbeva, P., Van Veen, J.A., and Van Elsas, J.D. (2004). Microbial diversity in soil: Selection of microbial populations by plant and soil type and 
implications for disease suppressiveness. Annual Review of Phytopathology 42, 243-270. doi: DOI 10.1146/annurev.phyto.42.012604.135455.

Gardner, A., and West, S.A. (2004). Spite and the scale of competition. Journal of Evolutionary Biology 17, 1195-1203. doi: 10.1111/j.1420.9101.2004.00775.x.

Giske, C.G., Monnet, D.L., Cars, O., and Carmeli, Y. (2008). Clinical and economic impact of common multidrug-resistant gram-negative bacilli. Antimicrob Agents Chemother 52, 813-821. doi: 10.1128/AAC.01169-07.

Glick, B.R. (1995). The enhancement of plant growth by free-living bacteria. Canadian Journal of Microbiology 41, 109-117. doi: 10.1139/m95-015.

Goh, E.B., Yim, G., Tsui, W., Mcclure, J., Surette, M.G., and Davies, J. (2002). Transcriptional modulation of bacterial gene expression by subinhibitory concentrations of antibiotics. Proceedings of the National Academy of Sciences of the United States of America 99, 17025-17030. doi: DOI 10.1073/pnas.252607699.

Gonzalez, J.E., and Keshavan, N.D. (2006). Messing with bacterial quorum sensing. Microbiology and Molecular Biology Reviews 70, 859-+. doi: Doi 10.1128/Mmbr.00002-06.

Groenhagen, U., Baumgartner, R., Bailly, A., Gardiner, A., Eberl, L., Schulz, S., and Weisskopf, L. (2013). Production of bioactive volatiles by different Burkholderia ambifaria strains. J Chem Ecol 39, 892-906. doi: 10.1007/s10886-013-0315-y.

Grosskopf, T., and Soyer, O.S. (2014). Synthetic microbial communities. Current opinion in microbiology 18, 72-77. doi: 10.1016/j.mib.2014.02.002.

Guzman, L.M., Belin, D., Carson, M.J., and Beckwith, J. (1995). Tight regulation, modulation, and high-level expression by vectors containing the arabinose PBAD promoter. Journal of Bacteriology 177, 4121-4130.

Haas, D., and Defago, G. (2005). Biological control of soil-borne pathogens by fluorescent pseudomonads. Nature Reviews Microbiology 3, 307-319. doi: $10.1038 /$ nrmicro1129.

Handelsman, J. (2005). How to find new antibiotics. Scientist 19, $20-21$.

Hardoim, P.R., Van Overbeek, L.S., and Elsas, J.D.V. (2008). Properties of bacterial endophytes and their proposed role in plant growth. Trends in Microbiology 16, 463-471. doi: http://dx.doi.org/10.1016/j.tim.2008.07.008.

Hayat, R., Ali, S., Amara, U., Khalid, R., and Ahmed, I. (2010). Soil beneficial bacteria and their role in plant growth promotion: a review. Annals of Microbiology 6o, 579-598. doi: 10.1007/s13213-010-0117-1. 
Hemaiswarya, S., and Doble, M. (2010). Synergistic interaction of phenylpropanoids with antibiotics against bacteria. Journal of Medical Microbiology 59, 1469-1476. doi: doi:10.1099/jmm.0.022426-o.

Hibbing, M.E., Fuqua, C., Parsek, M.R., and Peterson, S.B. (2010). Bacterial competition: surviving and thriving in the microbial jungle. Nature Reviews Microbiology 8, 15-25. doi: 10.1038/nrmicro2259.

Hoffman, L.R., D'argenio, D.A., Maccoss, M.J., Zhang, Z., Jones, R.A., and Miller, S.I. (2005). Aminoglycoside antibiotics induce bacterial biofilm formation. Nature 436, 1171-1175. doi: 10.1038/natureo3912.

Hsu, S.B., and Waltman, P. (1997). Competition between plasmid-bearing and plasmid-free organisms in selective media. Chemical Engineering Science 52, 23-35.

Hu, P., Janga, S.C., Babu, M., Díaz-Mejía, J.J., Butland, G., Yang, W., Pogoutse, O., Guo, X., Phanse, S., Wong, P., Chandran, S., Christopoulos, C., Nazarians-Armavil, A., Nasseri, N.K., Musso, G., Ali, M., Nazemof, N., Eroukova, V., Golshani, A., Paccanaro, A., Greenblatt, J.F., MorenoHagelsieb, G., and Emili, A. (2009). Global Functional Atlas of Escherichia coli Encompassing Previously Uncharacterized Proteins. PLoS Biol 7, elooooo96. doi: 10.1371/journal.pbio.1000096.

Huang, C.J., Tsay, J.F., Chang, S.Y., Yang, H.P., Wu, W.S., and Chen, C.Y. (2012). Dimethyl disulfide is an induced systemic resistance elicitor produced by Bacillus cereus C1L. Pest Management Science 68, 13061310. doi: 10.1002/ps.3301.

Huse, S.M., Dethlefsen, L., Huber, J.A., Welch, D.M., Relman, D.A., and Sogin, M.L. (2008). Exploring Microbial Diversity and Taxonomy Using SSU rRNA Hypervariable Tag Sequencing. Plos Genetics 4, e10oo255. doi: 10.1371/journal.pgen.1000255.

Ikeda, H., Ishikawa, J., Hanamoto, A., Shinose, M., Kikuchi, H., Shiba, T., Sakaki, Y., Hattori, M., and Omura, S. (2003). Complete genome sequence and comparative analysis of the industrial microorganism Streptomyces avermitilis. Nature Biotechnology 21, 526-531. doi: Doi $10.1038 / \mathrm{Nbt} 820$.

Inglis, R.F., Brown, S.P., and Buckling, A. (2012). Spite Versus Cheats: Competition among Social Strategies Shapes Virulence in Pseudomonas Aeruginosa. Evolution 66, 3472-3484. doi: DOI 10.1111/j.1558-5646.2012.01706.x.

Insam, H., and Seewald, M.A. (2010). Volatile organic compounds (VOCs) in soils. Biology and Fertility of Soils 46, 199-213. doi: 10.1007/soo374-0100442-3.

Ishige, T., Krause, M., Bott, M., Wendisch, V.F., and Sahm, H. (2003). The Phosphate Starvation Stimulon of Corynebacterium glutamicum Determined by DNA Microarray Analyses. Journal of Bacteriology 185, 4519-4529. doi: 10.1128/jb.185.15.4519-4529.2003. 
Jones, P., Binns, D., Chang, H.Y., Fraser, M., Li, W., Mcanulla, C., Mcwilliam, H., Maslen, J., Mitchell, A., Nuka, G., Pesseat, S., Quinn, A.F., Sangrador-Vegas, A., Scheremetjew, M., Yong, S.Y., Lopez, R., and Hunter, S. (2014). InterProScan 5: genome-scale protein function classification. Bioinformatics 30, 1236-1240. doi: 10.1093/bioinformatics/btuo31.

Jousset, A., Schmid, B., Scheu, S., and Eisenhauer, N. (2011). Genotypic richness and dissimilarity opposingly affect ecosystem functioning. Ecology Letters 14, 537-545. doi: 10.1111/j.1461-0248.2011.01613.x.

Kai, M., Haustein, M., Molina, F., Petri, A., Scholz, B., and Piechulla, B. (2009). Bacterial volatiles and their action potential. Appl Microbiol Biotechnol 81, 1001-1012. doi: 10.1007/soo253-008-176o-3.

Kamath, A.V., and Vaidyanathan, C.S. (1990). New Pathway for the Biodegradation of Indole in Aspergillus-Niger. Applied and Environmental Microbiology 56, 275-280.

Kanai, K., Shibayama, K., Suzuki, S., Wachino, J.I., and Arakawa, Y. (2004). Growth competition of macrolide-resistant and susceptible Helicobacter pylori strains. Microbiology and Immunology 48, 977980.

Kanchiswamy, C.N., Malnoy, M., and Maffei, M.E. (2015). Chemical diversity of microbial volatiles and their potential for plant growth and productivity. Frontiers in Plant Science 6. doi: 10.3389/fpls.2015.00151.

Katajamaa, M., Miettinen, J., and Oresic, M. (2006). MZmine: toolbox for processing and visualization of mass spectrometry based molecular profile data. Bioinformatics 22, 634-636. doi: 10.1093/bioinformatics/btko39.

Keller, L., and Surette, M.G. (2006). Communication in bacteria: an ecological and evolutionary perspective. Nature Reviews Microbiology 4, 249258. doi: $10.1038 /$ nrmicro1383.

Kern, W.V. (2006). Daptomycin: first in a new class of antibiotics for complicated skin and soft-tissue infections. International Journal of Clinical Practice 6o, 370-378. doi: 10.1111/j.1368-5031.2005.00885.x.

Kerr, B., Riley, M.A., Feldman, M.W., and Bohannan, B.J.M. (2002). Local dispersal promotes biodiversity in a real-life game of rock-paperscissors. Nature 418, 171-174.

Kim, K.S., Lee, S., and Ryu, C.M. (2013). Interspecific bacterial sensing through airborne signals modulates locomotion and drug resistance. Nature Communications 4, 1809. doi: 10.1038/ncomms2789.

Kinkel, L.L., Schlatter, D.C., Xiao, K., and Baines, A.D. (2014). Sympatric inhibition and niche differentiation suggest alternative coevolutionary trajectories among Streptomycetes. Isme Journal 8, 249-256. doi: 10.1038/ismej.2013.175.

Korpi, A., Pasanen, A.L., and Pasanen, P. (1998). Volatile compounds originating from mixed microbial cultures on building materials 
under various humidity conditions. Appl Environ Microbiol 64, 29142919.

Kova, J., Imunovi, K., Wu, Z., Klan?Nik, A., Bucar, F., Zhang, Q., and Mo?Ina, S.S. (2015). Antibiotic Resistance Modulation and Modes of Action of (-)- $\alpha$-Pinene in Campylobacter jejuni. Plos One 10, eo122871. doi: 10.1371/journal.pone.0122871.

Krings, U., and Berger, R.G. (1998). Biotechnological production of flavours and fragrances. Applied Microbiology and Biotechnology 49, 1-8.

Kucerova-Chlupacova, M., Kunes, J., Buchta, V., Vejsova, M., and Opletalova, V. (2015). Novel pyrazine analogs of chalcones: synthesis and evaluation of their antifungal and antimycobacterial activity. Molecules 20, 1104-1117. doi: 10.3390/molecules20011104.

Kugelberg, E., Lofmark, S., Wretlind, B., and Andersson, D.I. (2005). Reduction of the fitness burden of quinolone resistance in Pseudomonas aeruginosa. Journal of Antimicrobial Chemotherapy 55, 22-30. doi: 10.1093/jac/dkh505.

Lambers, H., Mougel, C., Jaillard, B., and Hinsinger, P. (2009). Plant-microbesoil interactions in the rhizosphere: an evolutionary perspective. Plant and Soil 321, 83-115. doi: 10.1007/s11104-009-0042-x.

Lane, D.J. (1991). 16S/23S rRNA sequencing. In: Nucleic acid techniques in bacterial systematics. New York, NY: John Wiley and Sons.

Langmead, B., and Salzberg, S.L. (2012). Fast gapped-read alignment with Bowtie 2. Nat Meth 9, 357-359. doi: 10.1038/nmeth.1923.

Lee, J.-H., Wood, T.K., and Lee, J. (2015). Roles of Indole as an Interspecies and Interkingdom Signaling Molecule. Trends in Microbiology 23, 707718. doi: http://dx.doi.org/10.1016/j.tim.2015.08.001.

Lee, J., Attila, C., Cirillo, S.L.G., Cirillo, J.D., and Wood, T.K. (2009). Indole and 7-hydroxyindole diminish Pseudomonas aeruginosa virulence. Microbial Biotechnology 2, 75-90. doi: 10.1111/j.1751-7915.2008.00061.x.

Lee, J.H., and Lee, J. (2010). Indole as an intercellular signal in microbial communities. Fems Microbiology Reviews 34, 426-444. doi: 10.1111/j.1574-6976.2009.00204.X.

Leedjarv, A., Ivask, A., Virta, M., and Kahru, A. (2006). Analysis of bioavailable phenols from natural samples by recombinant luminescent bacterial sensors. Chemosphere 64, 1910-1919. doi: 10.1016/j.chemosphere.2006.01.026.

Lemfack, M.C., Nickel, J., Dunkel, M., Preissner, R., and Piechulla, B. (2014). mVOC: a database of microbial volatiles. Nucleic Acids Research 42, D744-748. doi: 10.1093/nar/gkt1250.

Letoffe, S., Audrain, B., Bernier, S.P., Delepierre, M., and Ghigo, J.-M. (2014). Aerial Exposure to the Bacterial Volatile Compound Trimethylamine Modifies Antibiotic Resistance of Physically Separated Bacteria by Raising Culture Medium pH. Mbio 5. doi: 10.1128/mBio.00944-13. 
Li, B., and Dewey, C.N. (2011). RSEM: accurate transcript quantification from RNA-Seq data with or without a reference genome. Bmc Bioinformatics 12, 1-16. doi: 10.1186/1471-2105-12-323.

Li, Q., Ning, P., Zheng, L., Huang, J., Li, G., and Hsiang, T. (2010). Fumigant activity of volatiles of Streptomyces globisporus JK-1 against Penicillium italicum on Citrus microcarpa. Postharvest Biology and Technology 58, 157-165. doi: http://dx.doi.org/10.1016/j.postharvbio.2010.06.003.

Linares, J.F., Gustafsson, I., Baquero, F., and Martinez, J.L. (2006). Antibiotics as intermicrobial signaling agents instead of weapons. Proceedings of the National Academy of Sciences of the United States of America 103, 19484-19489. doi: DOI 10.1073/pnas.0608949103.

Livak, K.J., and Schmittgen, T.D. (2001). Analysis of Relative Gene Expression Data Using Real-Time Quantitative PCR and the 2- $\Delta \Delta C T$ Method. Methods 25, 402-408. doi: http://dx.doi.org/10.1006/meth.2001.1262.

Livermore, D. (2004). The need for new antibiotics. Clinical Microbiology and Infection 10, 1-9.

Lyon, G.J., and Muir, T.W. (2003). Chemical signaling among bacteria and its inhibition. Chemistry \& Biology 10, 1007-1021. doi: DOI 10.1016/j.chembiol.2003.11.003.

Marmann, A., Aly, A., Lin, W., Wang, B., and Proksch, P. (2014). CoCultivation-A Powerful Emerging Tool for Enhancing the Chemical Diversity of Microorganisms. Marine Drugs 12, 1043.

Maurhofer, M., Baehler, E., Notz, R., Martinez, V., and Keel, C. (2004). Cross talk between 2,4-diacetylphloroglucinol-producing biocontrol pseudomonads on wheat roots. Applied and Environmental Microbiology 70, 1990-1998. doi: Doi 10.1128/Aem.70.4.1990-1998.2004.

Mcgregor, K.F., and Young, H.-K. (200o). Identification and Characterization ofvanB2 Glycopeptide Resistance Elements in Enterococci Isolated in Scotland. Antimicrobial Agents and Chemotherapy 44, 2341-2348. doi: 10.1128/aac.44.9.2341-2348.2000.

Mcspadden Gardener, B.B. (2004). Ecology of Bacillus and Paenibacillus spp. in Agricultural Systems. Phytopathology 94, 1252-1258. doi: 10.1094/phyto.2004.94.11.1252.

Medema, M.H., Blin, K., Cimermancic, P., De Jager, V., Zakrzewski, P., Fischbach, M.A., Weber, T., Takano, E., and Breitling, R. (2011). antiSMASH: rapid identification, annotation and analysis of secondary metabolite biosynthesis gene clusters in bacterial and fungal genome sequences. Nucleic Acids Research 39, W339-346. doi: $10.1093 / \mathrm{nar} / \mathrm{gkr} 466$.

Melnyk, A.H., Wong, A., and Kassen, R. (2015). The fitness costs of antibiotic resistance mutations. Evolutionary Applications 8, 273-283.

Mendes, R., Garbeva, P., and Raaijmakers, J.M. (2013). The rhizosphere microbiome: significance of plant beneficial, plant pathogenic, and 
human pathogenic microorganisms. Fems Microbiology Reviews 37, 634-663. doi: Doi 10.1111/1574-6976.12028.

Meyer, S.A., and Schleifer, K.H. (1978). Deoxyribonucleic acid reassociation in the classification of coagulase-positive staphylococci. Arch Microbiol $117,183-188$.

Mitri, S., and Foster, K.R. (2013). The genotypic view of social interactions in microbial communities. Annual Review of Genetics 47, 247-273. doi: 10.1146/annurev-genet-111212-133307.

Monier, J.M., Demaneche, S., Delmont, T.O., Mathieu, A., Vogel, T.M., and Simonet, P. (2011). Metagenomic exploration of antibiotic resistance in soil. Curr Opin Microbiol 14, 229-235. doi: 10.1016/j.mib.2011.04.010.

Morohoshi, T., Shiono, T., Takidouchi, K., Kato, M., Kato, N., Kato, J., and Ikeda, T. (2007). Inhibition of Quorum Sensing in Serratia marcescens AS-1 by Synthetic Analogs of N-Acylhomoserine Lactone. Applied and Environmental Microbiology 73, 6339-6344. doi: 10.1128/aem.00593-07.

Nikaido, E., Yamaguchi, A., and Nishino, K. (2008). AcrAB multidrug efflux pump regulation in Salmonella enterica serovar typhimurium by RamA in response to environmental signals. Journal of Biological Chemistry 283, 24245-24253. doi: 10.1074/jbc.M804544200.

Nkanga, E.J., and Hagedorn, C. (1978). Detection of Antibiotic-Producing Streptomyces Inhabiting Forest Soils. Antimicrobial Agents and Chemotherapy 14, 51-59.

Nowak, J., and Shulaev, V. (2003). Priming for transplant stress resistance in In vitro propagation. In Vitro Cellular \& Developmental Biology - Plant 39, 107-124. doi: 10.1079/ivp2002403.

Oerke, E.C. (2006). Crop losses to pests. The Journal of Agricultural Science 144, 31-43. doi: doi:10.1017/Soo21859605005708.

Onaka, H., Mori, Y., Igarashi, Y., and Furumai, T. (2011). Mycolic AcidContaining Bacteria Induce Natural-Product Biosynthesis in Streptomyces Species. Applied and Environmental Microbiology 77, 400-406. doi: Doi 10.1128/Aem.01337-10.

Ott, E.M., Muller, T., Muller, M., Franz, C., Ulrich, A., Gabel, M., and Seyfarth, W. (2001). Population dynamics and antagonistic potential of enterococci colonizing the phyllosphere of grasses. Journal of Applied Microbiology 91, 54-66.

Pantanella, F., Berlutti, F., Passariello, C., Sarli, S., Morea, C., and Schippa, S. (2007). Violacein and biofilm production in Janthinobacterium lividum. Journal of Applied Microbiology 102, 992-999. doi: DOI 10.1111/j.1365-2672.2006.03155.X.

Penuelas, J., Asensio, D., Tholl, D., Wenke, K., Rosenkranz, M., Piechulla, B., and Schnitzler, J.P. (2014). Biogenic volatile emissions from the soil. Plant Cell Environ 37, 1866-1891. doi: 10.1111/pce.12340.

Peters, M., Kilwinski, J., Beringhoff, A., Reckling, D., and Genersch, E. (2006). American foulbrood of the honey bee: Occurrence and distribution of 
different genotypes of Paenibacillus larvae in the administrative district of Arnsberg (North Rhine-Westphalia). Journal of Veterinary Medicine, Series B 53, 100-104. doi: 10.1111/j.1439-0450.2006.00920.x.

Picard, F., Loubière, P., Girbal, L., and Cocaign-Bousquet, M. (2013). The significance of translation regulation in the stress response. Bmc Genomics 14, 1-11. doi: 10.1186/1471-2164-14-588.

Piel, J. (2002). A polyketide synthase-peptide synthetase gene cluster from an uncultured bacterial symbiont of Paederus beetles. Proc Natl Acad Sci U S A 99, 14002-14007. doi: 10.1073/pnas.222481399.

Piel, J. (2009). Metabolites from symbiotic bacteria. Nat Prod Rep 26, 338-362. doi: 10.1039/b703499g.

Pluskal, T., Castillo, S., Villar-Briones, A., and Oresic, M. (2010). MZmine 2: modular framework for processing, visualizing, and analyzing mass spectrometry-based molecular profile data. Bmc Bioinformatics 11, 395. doi: 10.1186/1471-2105-11-395.

Popova, A.A., Koksharova, O.A., Lipasova, V.A., Zaitseva, J.V., KatkovaZhukotskaya, O.A., Eremina, S.I., Mironov, A.S., Chernin, L.S., and Khmel, I.A. (2014). Inhibitory and Toxic Effects of Volatiles Emitted by Strains of Pseudomonas and Serratia on Growth and Survival of Selected Microorganisms, Caenorhabditis elegans, and Drosophila melanogaster. Biomed Res Int. doi: Artn 125704 10.1155/2014/125704.

Raaijmakers, J.M., Bonsall, R.F., and Weller, D.M. (1999). Effect of Population Density of Pseudomonas fluorescens on Production of 2,4Diacetylphloroglucinol in the Rhizosphere of Wheat. Phytopathology 89, 470-475. doi: 10.1094/PHYTO.1999.89.6.470.

Raaijmakers, J.M., and Mazzola, M. (2012). Diversity and Natural Functions of Antibiotics Produced by Beneficial and Plant Pathogenic Bacteria. Annual Review of Phytopathology, Vol 50 50, 403-424. doi: DOI 10.1146/annurev-phyto-081211-172908.

Raaijmakers, J.M., Paulitz, T.C., Steinberg, C., Alabouvette, C., and MoenneLoccoz, Y. (2009). The rhizosphere: a playground and battlefield for soilborne pathogens and beneficial microorganisms. Plant and Soil 321, 341-361. doi: DOI 10.1007/s11104-0o8-9568-6.

Raaijmakers, J.M., Vlami, M., and De Souza, J.T. (2002). Antibiotic production by bacterial biocontrol agents. Antonie Van Leeuwenhoek International Journal of General and Molecular Microbiology 81, 537547.

Rajini, K.S., Aparna, P., Sasikala, C., and Ramana Ch, V. (2011). Microbial metabolism of pyrazines. Crit Rev Microbiol 37, 99-112. doi: 10.3109/1040841X.2010.512267.

Reinhard, F., and Van Der Meer, J.R. (2011). Microcolony growth assays. Microbiology of Hydrocarbons, Oils, Lipids. 
Reinhold-Hurek, B., and Hurek, T. (2011). Living inside plants: bacterial endophytes. Current Opinion in Plant Biology 14, 435-443. doi: http://dx.doi.org/10.1016/j.pbi.2011.04.004.

Rice, L.B. (2006). Antimicrobial resistance in gram-positive bacteria. American Journal of Infection Control 34, S11-19; discussion S64-73. doi: 10.1016/j.ajic.2006.05.220.

Richardson, A.E., and Simpson, R.J. (2011). Soil Microorganisms Mediating Phosphorus Availability Update on Microbial Phosphorus. Plant Physiology 156, 989-996. doi: 10.1104/pp.111.175448.

Riley, M.A., Goldstone, C.M., Wertz, J.E., and Gordon, D. (2003). A phylogenetic approach to assessing the targets of microbial warfare. Journal of Evolutionary Biology 16, 690-697. doi: DOI 10.1046/j.14209101.2003.00575.x.

Riley, M.A., and Wertz, J.E. (2002a). Bacteriocin diversity: ecological and evolutionary perspectives. Biochimie 84, 357-364.

Riley, M.A., and Wertz, J.E. (2002b). Bacteriocins: Evolution, ecology, and application. Annual Review of Microbiology 56, 117-137. doi: 10.1146/annurev.micro.56.012302.161024.

Robinson, M.D., Mccarthy, D.J., and Smyth, G.K. (2010). edgeR: a Bioconductor package for differential expression analysis of digital gene expression data. Bioinformatics 26. doi: 10.1093/bioinformatics/btp616.

Romero, D., Traxler, M.F., Lopez, D., and Kolter, R. (2011). Antibiotics as signal molecules. Chemical Reviews 111, 5492-5505. doi: 10.1021/cr2000509.

Romoli, R., Papaleo, M.C., De Pascale, D., Tutino, M.L., Michaud, L., Logiudice, A., Fani, R., and Bartolucci, G. (2014). GC-MS volatolomic approach to study the antimicrobial activity of the antarctic bacterium Pseudoalteromonas sp. TB41. Metabolomics 10, 42-51. doi: 10.1007/s11306-013-0549-2.

Rousk, J., and Baath, E. (2007). Fungal and bacterial growth in soil with plant materials of different C/N ratios. FEMS Microbiol Ecol 62, 258-267. doi: 10.1111/j.1574-6941.2007.00398.x.

Rousk, J., Demoling, L.A., and Baath, E. (2009). Contrasting Short-Term Antibiotic Effects on Respiration and Bacterial Growth Compromises the Validity of the Selective Respiratory Inhibition Technique to Distinguish Fungi and Bacteria. Microbial Ecology 58, 75-85. doi: DOI 10.1007/s00248-008-9444-1.

Ryu, C.M., Farag, M.A., Hu, C.H., Reddy, M.S., Kloepper, J.W., and Pare, P.W. (2004). Bacterial volatiles induce systemic resistance in Arabidopsis. Plant Physiology 134, 1017-1026.

Ryu, C.M., Farag, M.A., Hu, C.H., Reddy, M.S., Wei, H.X., Pare, P.W., and Kloepper, J.W. (2003). Bacterial volatiles promote growth in Arabidopsis. Proceedings of the National Academy of Sciences of the United States of America 100, 4927-4932. 
Ryu, C.M., Hu, C.H., Locy, R.D., and Kloepper, J.W. (2005). Study of mechanisms for plant growth promotion elicited by rhizobacteria in Arabidopsis thaliana. Plant and Soil 268, 285-292. doi: 10.1007/s11104004-0301-9.

Saleh, O., Bonitz, T., Flinspach, K., Kulik, A., Burkard, N., Muhlenweg, A., Vente, A., Polnick, S., Lammerhofer, M., Gust, B., Fiedler, H.P., and Heide, L. (2012). Activation of a silent phenazine biosynthetic gene cluster reveals a novel natural product and a new resistance mechanism against phenazines. Medchemcomm 3, 1009-1019. doi: Doi 10.1039/C2md20045g.

Salles, J.F., De Souza, F.A., and Van Elsas, J.D. (2002). Molecular Method To Assess the Diversity of Burkholderia Species in Environmental Samples. Applied and Environmental Microbiology 68, 1595-1603. doi: 10.1128/aem.68.4.1595-1603.2002.

Sambrook, J., and Russell, D.W. (2001). Molecular cloning: a laboratory manual. Cold spring harbor laboratory press.

Sanchez, S., Chavez, A., Forero, A., Garcia-Huante, Y., Romero, A., Sanchez, M., Rocha, D., Sanchez, B., Avalos, M., Guzman-Trampe, S., Rodriguez-Sanoja, R., Langley, E., and Ruiz, B. (2010). Carbon source regulation of antibiotic production. Journal of Antibiotics 63, 442-459.

Scherlach, K., and Hertweck, C. (2009). Triggering cryptic natural product biosynthesis in microorganisms. Organic E Biomolecular Chemistry 7, 1753-176o. doi: Doi 10.1039/B821578b.

Schmidt, A. (1996). Systemic candidiasis in Sprague-Dawley rats. J Med Vet Mycol 34, 99-104.

Schmidt, R., Cordovez, V., De Boer, W., Raaijmakers, J., and Garbeva, P. (2015). Volatile affairs in microbial interactions. ISME J. doi: $10.1038 /$ ismej.2015.42.

Schmidt, S., Blom, J.F., Pernthaler, J., Berg, G., Baldwin, A., Mahenthiralingam, E., and Eberl, L. (2009). Production of the antifungal compound pyrrolnitrin is quorum sensing-regulated in members of the Burkholderia cepacia complex. Environmental Microbiology 11, 1422-1437. doi: 10.1111/j.1462-2920.2009.01870.x.

Schneider, J., Yepes, A., Garcia-Betancur, J.C., Westedt, I., Mielich, B., and Lopez, D. (2012). Streptomycin-Induced Expression in Bacillus subtilis of YtnP, a Lactonase-Homologous Protein That Inhibits Development and Streptomycin Production in Streptomyces griseus. Applied and Environmental Microbiology 78, 599-603. doi: Doi 10.1128/Aem.0699211.

Schulz-Bohm, K., Zweers, H., De Boer, W., and Garbeva, P. (2015). A fragrant neighborhood: Volatile mediated bacterial interactions in soil. Frontiers in Microbiology 6. doi: 10.3389/fmicb.2015.01212.

Schulz, S., and Dickschat, J.S. (2007). Bacterial volatiles: the smell of small organisms. Nat Prod Rep 24, 814-842. doi: 10.1039/b507392h. 
Schulz, S., Fuhlendorff, J., and Reichenbach, H. (2004). Identification and synthesis of volatiles released by the myxobacterium Chondromyces crocatus. Tetrahedron 60, 3863-3872. doi: 10.1016/j.tet.2004.03.005.

Schwab, W., Davidovich-Rikanati, R., and Lewinsohn, E. (2008). Biosynthesis of plant-derived flavor compounds. Plant Journal 54, 712-732. doi: DOI 10.1111/j.1365-313X.2008.03446.x.

Seemann, T. (2014). Prokka: rapid prokaryotic genome annotation. Bioinformatics 30, 2068-2069. doi: 10.1093/bioinformatics/btu153.

Sengupta, S., Chattopadhyay, M.K., and Grossart, H.-P. (2013). The multifaceted roles of antibiotics and antibiotic resistance in nature. Frontiers in Microbiology 4. doi: 10.3389/fmicb.2013.00047.

Sessitsch, A., Coenye, T., Sturz, A.V., Vandamme, P., Barka, E.A., Salles, J.F., Van Elsas, J.D., Faure, D., Reiter, B., Glick, B.R., Wang-Pruski, G., and Nowak, J. (2005). Burkholderia phytofirmans sp. nov., a novel plantassociated bacterium with plant-beneficial properties. International Journal of Systematic and Evolutionary Microbiology 55, 1187-1192. doi: doi:10.1099/ijs.o.63149-o.

Seyedsayamdost, M.R., Traxler, M.F., Clardy, J., and Kolter, R. (2012). Old Meets New: Using Interspecies Interactions to Detect Secondary Metabolite Production in Actinomycetes. Natural Product Biosynthesis by Microorganisms and Plants, Pt C 517, 89-109. doi: Doi 10.1016/B978-0-12-404634-4.00005-X.

Shannon, P., Markiel, A., Ozier, O., Baliga, N.S., Wang, J.T., Ramage, D., Amin, N., Schwikowski, B., and Ideker, T. (2003). Cytoscape: A software environment for integrated models of biomolecular interaction networks. Genome Research 13, 2498-2504. doi: Doi 10.1101/Gr.1239303.

Shimada, Y., Kinoshita, M., Harada, K., Mizutani, M., Masahata, K., Kayama, H., and Takeda, K. (2013). Commensal Bacteria-Dependent Indole Production Enhances Epithelial Barrier Function in the Colon. Plos One 8. doi: ARTN e80604 10.1371/journal.pone.0080604.

Shong, J., Jimenez Diaz, M.R., and Collins, C.H. (2012). Towards synthetic microbial consortia for bioprocessing. Curr Opin Biotechnol 23, 798802. doi: 10.1016/j.copbio.2012.02.001.

Silberbach, M., and Burkovski, A. (2006). Application of global analysis techniques to Corynebacterium glutamicum: New insights into nitrogen regulation. Journal of Biotechnology 126, 101-110. doi: http://dx.doi.org/10.1016/j.jbiotec.2006.03.039.

Slattery, M., Rajbhandari, I., and Wesson, K. (2001). Competition-mediated antibiotic induction in the marine bacterium Streptomyces tenjimariensis. Microbial Ecology 41, 90-96.

Snel, B., Bork, P., and Huynen, M.A. (2002). The identification of functional modules from the genomic association of genes. Proc Natl Acad Sci U S A 99, 5890-5895. doi: 10.1073/pnas.092632599. 
Song, C., Schmidt, R., De Jager, V., Krzyzanowska, D., Jongedijk, E., Cankar, K., Beekwilder, J., Van Veen, A., De Boer, W., Van Veen, J.A., and Garbeva, P. (2015). Exploring the genomic traits of fungus-feeding bacterial genus Collimonas. Bmc Genomics 16, 1103. doi: 10.1186/s12864-015-2289-3.

Spellberg, B., Guidos, R., Gilbert, D., Bradley, J., Boucher, H.W., Scheld, W.M., Bartlett, J.G., Edwards, J., and Amer, I.D.S. (2008). The epidemic of antibiotic-resistant infections: A call to action for the medical community from the Infectious Diseases Society of America. Clinical Infectious Diseases 46, 155-164. doi: Doi 10.1086/524891.

Straight, P.D., Fischbach, M.A., Walsh, C.T., Rudner, D.Z., and Kolter, R. (2007). A singular enzymatic megacomplex from Bacillus subtilis. Proceedings of the National Academy of Sciences of the United States of America 104, 305-310. doi: DOI 10.1073/pnas.0609073103.

Straight, P.D., Willey, J.M., and Kolter, R. (2006). Interactions between Streptomyces coelicolor and Bacillus subtilis: Role of Surfactants in Raising Aerial Structures. Journal of Bacteriology 188, 4918-4925. doi: 10.1128/jb.00162-o6.

Strickland, M.S., C. Lauber Et Al. (2009). Testing the functional significance of microbial community composition. Ecology 90, 441-451.

Tait, E., Perry, J.D., Stanforth, S.P., and Dean, J.R. (2014). Identification of volatile organic compounds produced by bacteria using HS-SPMEGC-MS. Journal of Chromatographic Science 52, 363-373. doi: 10.1093/chromsci/bmto42.

Tecon, R., Beggah, S., Czechowska, K., Sentchilo, V., Chronopoulou, P.M., Mcgenity, T.J., and Van Der Meer, J.R. (2010). Development of a multistrain bacterial bioreporter platform for the monitoring of hydrocarbon contaminants in marine environments. Environmental Science \& Technology 44, 1049-1055. doi: 10.1021/es902849w.

Tenover, F.C., Biddle, J.W., and Lancaster, M.V. (2001). Increasing resistance to vancomycin and other glycopeptides in Staphylococcus aureus. Emerging Infectious Diseases 7, 327-332.

Timmusk, S., Paalme, V., Lagercrantz, U., and Nevo, E. (2009). Detection and quantification of Paenibacillus polymyxa in the rhizosphere of wild barley (Hordeum spontaneum) with real-time PCR. Journal of Applied Microbiology 107, 736-745. doi: 10.1111/j.1365-2672.2009.04265.x.

Torsvik, V., Goksoyr, J., and Daae, F.L. (1990a). High diversity in DNA of soil bacteria. Appl Environ Microbiol 56, 782-787.

Torsvik, V., and Ovreas, L. (2002). Microbial diversity and function in soil: from genes to ecosystems. Curr Opin Microbiol 5, 240-245. doi: S1369527402003247 [pii].

Torsvik, V., Salte, K., Sorheim, R., and Goksoyr, J. (199ob). Comparison of phenotypic diversity and DNA heterogeneity in a population of soil bacteria. Appl Environ Microbiol 56, 776-781. 
Traxler, M.F., Watrous, J.D., Alexandrov, T., Dorrestein, P.C., and Kolter, R. (2013). Interspecies Interactions Stimulate Diversification of the Streptomyces coelicolor Secreted Metabolome. Mbio 4. doi: DOI 10.1128/mBio.00459-13.

Tyc, O., Van Den Berg, M., Gerards, S., Van Veen, J.A., Raaijmakers, J.M., De Boer, W., and Garbeva, P. (2014). Impact of interspecific interactions on antimicrobial activity among soil bacteria. Frontiers in Microbiology 5, 567. doi: 10.3389/fmicb.2014.00567.

Tyc, O., Wolf, A.B., and Garbeva, P. (2015). The Effect of Phylogenetically Different Bacteria on the Fitness of Pseudomonas fluorescens in Sand Microcosms. Plos One 10. doi: ARTN eo119838 10.1371/journal.pone.0119838.

Ueda, K., Kawai, S., Ogawa, H., Kiyama, A., Kubota, T., Kawanobe, H., and Beppu, T. (2000). Wide distribution of interspecific stimulatory events on antibiotic production and sporulation among Streptomyces species. Journal of Antibiotics 53, 979-982.

Uroz, S., Buee, M., Murat, C., Frey-Klett, P., and Martin, F. (2010). Pyrosequencing reveals a contrasted bacterial diversity between oak rhizosphere and surrounding soil. Environmental Microbiology Reports 2, 281-288. doi: DOI 10.1111/j.1758-2229.2009.00117.x.

Van Agtmaal, M., Van Os, G., Hol, G., Hundscheid, M., Runia, W., Hordijk, C., and De Boer, W. (2015). Legacy effects of anaerobic soil disinfestation on soil bacterial community composition and production of pathogen-suppressing volatiles. Frontiers in Microbiology 6. doi: 10.3389/fmicb.2015.00701.

Van Den Berg, H.A., Orlov, M.V., and Kiselëv, Y.N. (2008). The Malthusian parameter in microbial ecology and evolution: An optimal control treatment. Computational Mathematics and Modeling 19, 406-428. doi: 10.1007/s10598-008-9013-y.

Van Der Burg, B., Pieterse, B., Buist, H., Lewin, G., Van Der Linden, S.C., Man, H.Y., Rorije, E., Piersma, A.H., Mangelsdorf, I., Wolterbeek, A.P., Kroese, E.D., and Van Vugt-Lussenburg, B. (2015). A high throughput screening system for predicting chemically-induced reproductive organ deformities. Reprod Toxicol 55, 95-103. doi: 10.1016/j.reprotox.2014.11.011.

Van Der Burg, B., Van Der Linden, S., Man, H.-Y., Winter, R., Jonker, L., Van Vugt-Lussenburg, B., and Brouwer, A. (2013). "A Panel of Quantitative Calux ${ }^{\circledast}$ Reporter Gene Assays for Reliable High-Throughput Toxicity Screening of Chemicals and Complex Mixtures," in High-Throughput Screening Methods in Toxicity Testing. John Wiley \& Sons, Inc.), 519532.

Van Der Heijden, M.G.A., Bardgett, R.D., and Van Straalen, N.M. (2008). The unseen majority: soil microbes as drivers of plant diversity and 
productivity in terrestrial ecosystems. Ecology Letters 11, 296-310. doi: 10.1111/j.1461-0248.2007.01139.x.

Van Elsas, J.D., Garbeva, P., and Salles, J. (2002). Effects of agronomical measures on the microbial diversity of soils as related to the suppression of soil-borne plant pathogens. Biodegradation 13, 29-40.

Van Wezel, G.P., and Mcdowall, K.J. (2011). The regulation of the secondary metabolism of Streptomyces: new links and experimental advances. Natural Product Reports 28, 1311-1333. doi: Doi 10.1039/Cinpooooza.

Vasi, F., Travisano, M., and Lenski, R.E. (1994). Long-Term Experimental Evolution in Escherichia coli. II. Changes in Life-History Traits During Adaptation to a Seasonal Environment. The American Naturalist 144, 432-456. doi: 10.2307/2462954.

Vaz Jauri, P., Bakker, M.G., Salomon, C.E., and Kinkel, L.L. (2013). Subinhibitory Antibiotic Concentrations Mediate Nutrient Use and Competition among Soil Streptomyces. Plos One 8, e81064. doi: 10.1371/journal.pone.0081064.

Velicer, G.J. (2003). Social strife in the microbial world. Trends in Microbiology 11, 330-337. doi: http://dx.doi.org/10.1016/Sog66-842X(03)o0152-5.

Venturi, V., and Subramoni, S. (2009). Future research trends in the major chemical language of bacteria. Hfsp Journal 3, 105-116. doi: Doi 10.2976/1.3065673.

Vial, L., Chapalain, A., Groleau, M.-C., and Déziel, E. (2011). The various lifestyles of the Burkholderia cepacia complex species: a tribute to adaptation. Environmental Microbiology 13, 1-12. doi: 10.1111/j.14622920.2010.02343.X.

Von Der Weid, I., Paiva, E., Nóbrega, A., Dirk Van Elsas, J., and Seldin, L. (200o). Diversity of Paenibacillus polymyxa strains isolated from the rhizosphere of maize planted in Cerrado soil. Research in Microbiology 151, 369-381. doi: http://dx.doi.org/10.1016/So9232508(00)00160-1.

Vos, M., and Velicer, G.J. (2009). Social Conflict in Centimeter and GlobalScale Populations of the Bacterium Myxococcus xanthus. Current Biology 19, 1763-1767. doi: 10.1016/j.cub.2009.08.061.

Walsh, F., Amyes, S.G., and Duffy, B. (2013). Challenging the concept of bacteria subsisting on antibiotics. Int J Antimicrob Agents 41, 558-563. doi: 10.1016/j.ijantimicag.2013.01.021.

Wang, C., Wang, Z., Qiao, X., Li, Z., Li, F., Chen, M., Wang, Y., Huang, Y., and Cui, H. (2013). Antifungal activity of volatile organic compounds from Streptomyces alboflavus TD-1. Fems Microbiology Letters 341, 45-51. doi: 10.1111/1574-6968.12088.

Wang, D.D., Ding, X.D., and Rather, P.N. (2001). Indole can act as an extracellular signal in Escherichia coli. Journal of Bacteriology 183, 4210-4216. doi: Doi 10.1128/Jb.183.14.4210-4216.2001. 
Weller, D.M., Raaijmakers, J.M., Gardener, B.B.M., and Thomashow, L.S. (2002). Microbial populations responsible for specific soil suppressiveness to plant pathogens. Annual Review of Phytopathology 40, 309-+. doi: DOI 10.1146/annurev.phyto.40.030402.110010.

Wellington, E.M., Boxall, A.B., Cross, P., Feil, E.J., Gaze, W.H., Hawkey, P.M., Johnson-Rollings, A.S., Jones, D.L., Lee, N.M., Otten, W., Thomas, C.M., and Williams, A.P. (2013). The role of the natural environment in the emergence of antibiotic resistance in gram-negative bacteria. Lancet Infectious Diseases 13, 155-165. doi: 10.1016/S1473-3099(12)703171.

Wenke, K., Kai, M., and Piechulla, B. (2010). Belowground volatiles facilitate interactions between plant roots and soil organisms. Planta 231, 499506. doi: 10.1007/s00425-009-1076-2.

Wheatley, R.E. (2002). The consequences of volatile organic compound mediated bacterial and fungal interactions. Antonie Van Leeuwenhoek $81,357-364$.

Workentine, M.L., Harrison, J.J., Weljie, A.M., Tran, V.A., Stenroos, P.U., Tremaroli, V., Vogel, H.J., Ceri, H., and Turner, R.J. (2010). Phenotypic and metabolic profiling of colony morphology variants evolved from Pseudomonas fluorescens biofilms. Environmental Microbiology 12, 1565-1577. doi: 10.1111/j.1462-2920.2010.02185.x.

Wu, X.-C., Shen, X.-B., Ding, R., Qian, C.-D., Fang, H.-H., and Li, O. (2010). Isolation and partial characterization of antibiotics produced by Paenibacillus elgii B69. Fems Microbiology Letters 310, 32-38. doi: 10.1111/j.1574-6968.2010.02040.x.

Xia, J., Mandal, R., Sinelnikov, I.V., Broadhurst, D., and Wishart, D.S. (2012). MetaboAnalyst 2.0--a comprehensive server for metabolomic data analysis. Nucleic Acids Research 40, W127-133. doi: 10.1093/nar/gks374.

Xia, J., Sinelnikov, I.V., Han, B., and Wishart, D.S. (2015). MetaboAnalyst 3.omaking metabolomics more meaningful. Nucleic Acids Research 43, W251-257. doi: 10.1093/nar/gkv38o.

Yamaguchi, S., and Yokoe, M. (200o). A novel protein-deamidating enzyme from Chryseobacterium proteolyticum sp nov., a newly isolated bacterium from soil. Applied and Environmental Microbiology 66, 3337-3343. doi: Doi 10.1128/Aem.66.8.3337-3343.2000.

Yim, G., Mcclure, J., Surette, M.G., and Davies, J.E. (2011). Modulation of Salmonella gene expression by subinhibitory concentrations of quinolones. Journal of Antibiotics 64, 73-78.

Yin, B., D. Crowley, Et Al. (200o). Bacterial functional redundancy along a soil reclamation gradient. Applied and Environmental Microbiology 66, 4361-4365.

Zhang, Q.J., Sahin, O., Mcdermott, P.F., and Payot, S. (2006). Fitness of antimicrobial-resistant Campylobacter and Salmonella. Microbes and Infection 8, 1972-1978. doi: 10.1016/j.micinf.2005.12.031. 
Zhang, R., Lipuma, J.J., and Gonzalez, C.F. (2009). Two type IV secretion systems with different functions in Burkholderia cenocepacia K56-2. Microbiology 155, 4005-4013. doi: 10.1099/mic.0.033043-0.

Zhao, K., Penttinen, P., Zhang, X., Ao, X., Liu, M., Yu, X., and Chen, Q. (2014). Maize rhizosphere in Sichuan, China, hosts plant growth promoting Burkholderia cepacia with phosphate solubilizing and antifungal abilities. Microbiological Research 169, 76-82. doi: http://dx.doi.org/10.1016/j.micres.2013.07.003.

Zhou, X.B., Lindsay, H., and Robinson, M.D. (2014). Robustly detecting differential expression in RNA sequencing data using observation weights. Nucleic Acids Research 42. doi: ARTN e9110.1093/nar/gkuz10.

Zhu, H. (2014). Environmental and Metabolomic Study of Antibiotic Production by Actinomycetes. PhD Thesis, Leiden University, The Netherlands.

Zou, C.S., Mo, M.H., Gu, Y.Q., Zhou, J.P., and Zhang, K.Q. (2007). Possible contributions of volatile-producing bacteria to soil fungistasis. Soil Biology \& Biochemistry 39, 2371-2379. doi: 10.1016/j.soilbio.2007.04.009. 


\section{Summary}




\section{Summary}

The soil habitat is one of the most important resources of microbial natural products of human interest such as antibiotics, enzymes and other secondary metabolites. Soil is a very complex environment where many microorganisms are constantly competing for limiting nutrients and space. So far, only a small fraction of the terrestrial bacterial species has been explored for novel pharmaceutical compounds. Facing the worldwide problem of increasing antibiotic resistance in pathogenic bacteria, novel antimicrobials are urgently needed. New methods and screening strategies are needed to access the full range of antimicrobials produced by terrestrial microbes. Current screening methods for discovering bioactive compounds do often target only well-examined genera that are known to produce antibiotics in monocultures. Such screening methods do not consider the importance of interspecific interactions, which represent the natural situation in which microorganisms produce and use antibiotics. This thesis is focused on competitive bacterial interactions with the aim to explore soil bacteria for novel antimicrobial compounds produced resulting from interspecific interactions.

A major objective of this thesis was to study the frequency of interspecific interaction induced antibiotic production in soil bacteria. For this purpose a high-through-put screening method was developed (chapter two) to screen soil bacteria for their antibiotic activity in monocultures and pairwise cultures. The results indicate clearly that interspecific interaction mediated induction of antibiotic activity is commonly occurring among soil bacteria. From all tested bacterial isolates $42 \%$ showed antimicrobial activity only during interactions and $33 \%$ of all tested isolates showed antimicrobial activity only in monoculture. Furthermore, the results indicated that the identity of the interacting partner is an important factor in the induction of antibiotic production. Besides induction of antibiotic production also suppression of antibiotic production was observed for $22 \%$ of all tested interactions and only $13 \%$ of all tested isolates showed antimicrobial activity in both monocultures and mixed cultures.

More detailed studies of the effect of interspecific interactions on the production of secondary metabolites by soil bacteria are presented in chapter three and four. Particular attention was given to the production of volatile organic compounds. 
Volatile blends emitted by phylogenetically different soil-bacteria grown either in monoculture or mixed cultures were compared. The results revealed that the volatile blends emitted during interactions differed from the volatile blends emitted by the respective monocultures. Interestingly some compounds that were produced by monocultures were not detected in the mixtures. Furthermore, the effect of bacterial volatiles on performance of target microbes (bacteria, fungi and oomycetes) was investigated. The results revealed that volatiles produced by bacteria can inhibit the growth of other bacteria, influence their colony morphology and can inhibit the growth of fungi and oomycetes. Interestingly, also the induction of volatile compound production (2,5-bis(1-methylethyl)-pyrazine) was observed (chapter four). This volatile compound was found to be produced in higher abundance during co-cultivation of Burkholderia sp. AD24 and Paenibacillus sp. AD87. The effect of interspecific interactions on the fitness, gene expression and on the production of soluble metabolites in soil bacteria was also studied. The results of chapter four revealed that the interaction between Burkholderia sp. AD24 and Paenibacillus sp. AD87 had a negative effect on numbers of Burkholderia sp. AD24 whereas those of Paenibacillus sp. AD87 were not affected.

A similar observation was described in chapter five. Here the growth of $P$. fluorescens Pfo-1 was negatively affected when confronted with Bacillus sp. V102. The additional performed transcriptome analysis in chapter four revealed differential expression of several genes related to ribosomal proteins, signal transduction, secondary metabolite production, antibiotic resistance, defense mechanisms and cell motility. The metabolomic analysis performed on soluble secondary metabolites revealed that the produced metabolites of monocultures of Burkholderia sp. AD24 and Paenibacillus sp. AD87 differed from the metabolites produced during interactions. Interaction-mediated triggering of antibiotic production provides a new opportunity to estimate the biological costs for the production of antimicrobial compounds. I investigated the possible costs for interaction-mediated antimicrobial compound production in P. fluorescens Pfo-1 by monitoring the growth rate with and without induction of antibiotic production. The results of this study are reported in chapter six and revealed that the production of an antimicrobial compound by $P$. fluorescens Pfo-1 did not incur detectable metabolic costs. 
In summary this thesis extends our knowledge on the impact of interspecific interactions on secondary metabolite production in soil bacteria thereby providing better insight in the competitive mechanisms occurring in soil microbial communities. This thesis further highlights the influence of interactions between Gram-negative and Gram-positive bacteria on bacterial fitness and on the production of volatile and soluble secondary metabolites. Furthermore, the thesis research strengthens the importance of interspecific microbial interactions for the discovery of novel antibiotics. 


\section{Samenvatting}




\section{Samenvatting}

De bodem is een van de belangrijkste bronnen van microbiële natuurlijke producten die toegepast worden voor industriële of therapeutische doeleinden. Voorbeelden zijn antibiotica en enzymen. De bodem is een zeer complexe omgeving waar micro-organismen voortdurend met elkaar concurreren om beperkt beschikbare voedingsstoffen en ruimte. Hierbij worden secundaire metabolieten ingezet om concurrenten te onderdrukken. Slechts een klein deel van de secundaire metabolieten die door bodembacteriën geproduceerd kunnen worden is onderzocht voor toepassingsdoeleinden. Om het wereldwijde probleem van toenemende resistentie in pathogene bacteriën tegen de algemeen gebruikte antibiotica te kunnen oplossen zijn nieuwe antibiotica dringend nodig. Ontwikkeling van nieuwe screeningsmethoden- is dan ook essentieel om het volledige potentieel aan antibiotische stoffen in bodembacteriën te ontdekken. De huidige screeningsmethoden voor het ontdekken van bioactieve stoffen richten zich alleen op bacteriële soorten waarvan bekend is dat ze antibiotica produceren in een monocultuur. Dergelijke screeningsmethoden houden geen rekening met competitieve interacties tussen microbiële soorten, terwijl dit de natuurlijke situatie is waarin micro-organismen antibiotica produceren en gebruiken. Met het in dit proefschrift beschreven onderzoek heb ik geprobeerd om vanuit een ecologische invalshoek strategieën te ontwikkelen die kunnen leiden tot ontdekking van nieuwe antibiotica. Daarvoor zijn interspecifieke interacties tussen bodembacteriën als uitgangspunt genomen.

Als eerste is de frequentie van geïnduceerde productie van antibiotica onderzocht voor een groep van bodembacteriën. Voor dit doel werd een highthrough-put screening methode ontwikkeld (hoofdstuk twee) om bodembacteriën te screenen op hun antibiotische activiteit in zowel monocultuur als gemengde (paarsgewijze) kweken. De resultaten gaven duidelijk aan dat inductie van antibiotica productie door interspecifieke interactie vaak optreedt bij bodembacteriën. Van alle geteste bacteriële isolaten toonde $42 \%$ alleen antimicrobiële activiteit tijdens interacties. Het onderzoek gaf echter ook aan dat de identiteit van de interacterende partner een belangrijke factor is bij de inductie van antibioticaproductie. Naast de inductie van antibioticaproductie heb ik ook onderdrukking van antibioticaproductie in gemengde kweken waargenomen. 
In hoofdstuk drie en vier worden meer gedetailleerde studies van het effect van interspecifieke interacties op de productie van secundaire metabolieten door bodembacteriën beschreven. Bijzondere aandacht werd hierbij besteed aan de productie van vluchtige organische stoffen door verschillende soorten bodembacteriën in monocultuur en gemengde kweken. Uit de resultaten bleek dat de samenstelling van vluchtige stoffen die vrijkomt in mengkweken verschilde van die in monoculturen Een aantal verbindingen die werden geproduceerd door monoculturen werden niet meer gedetecteerd in mengculturen. Daarnaast is het effect van bacteriële vluchtige stoffen op de groei van doelmicroben (bacteriën, schimmels en oömyceten) onderzocht. Uit de resultaten bleek dat vluchtige stoffen afkomstig van bacteriekweken de groei van andere bacteriën kunnen remmen, de kolonie morfologie kunnen beïnvloeden en de groei van schimmels en oomyceten kunnen remmen. Opvallend is ook de inductie van de productie van een vluchtige verbinding (2,5-bis(1-methylethyl)-pyrazine) (hoofdstuk vier). De productie van deze vluchtige verbinding nam sterk toe tijdens de interactie van Burkholderia sp. AD24 met Paenibacillus sp. AD87.

Het effect van interspecifieke bacteriële interacties op fitness, genexpressie en productie van oplosbare metabolieten werd ook bestudeerd. Uit de resultaten van hoofdstuk vier bleek dat de interactie tussen Burkholderia sp. AD24 en Paenibacillus sp. AD87 een negatief effect had op de Burkholderia aantallen, terwijl die van Paenibacillus niet werden beïnvloed. Een soortgelijke waarneming is beschreven in hoofdstuk vijf. Hier werd de groei van Pseudomonas fluorescens Pfo-1 negatief beïnvloed tijdens confrontatie met Bacillus sp. V102. Uit de aanvullende transcriptoom analyse in hoofdstuk vier bleek er sprake te zijn van differentiële expressie van verschillende genen betrokken bij ribosomale eiwit productie, signaaltransductie, secundaire metaboliet productie, resistentie tegen antibiotica, afweermechanismen en celmotiliteit. Uit de metaboloom analyse uitgevoerd op oplosbare secundaire metabolieten, is gebleken dat de geproduceerde metabolieten van monoculturen van Burkholderia sp. AD24 en Paenibacillus sp. AD87 verschillend zijn van de metabolieten geproduceerd in mengkweken.

De activering van antibioticaproductie tijdens interacties biedt een nieuwe kans om de biologische kosten voor de productie van antimicrobiële verbindingen te schatten. Ik onderzocht de mogelijke kosten voor interactie gemediëerde antibiotica productie in $P$. fluorescens Pfo-1 door het kwantificeren van de groei met en zonder inductie van antibiotica productie. 
De resultaten van dit onderzoek zijn beschreven in hoofdstuk zes en gaven aandat de productie van een antimicrobiële verbinding door $P$. fluorescens Pfo-1 niet detecteerbare metabole kosten met zich meebrengen.

Kort samengevat. Dit proefschrift vergroot onze kennis over (1) de gevolgen van interspecifieke interacties op de productie van secundaire metabolieten in bodem bacteriën en (2) de concurrerende mechanismen die in bodem microbiële gemeenschappen voorkomen. Dit proefschrift onderstreept het belang van verder onderzoek naar de invloed van interacties tussen Gram-negatieve en Gram-positieve bacteriën. Uit dit proefschrift komt naar voren dat het onderzoek naar interspecifieke microbiële interacties een belangrijke bijdrage kan leveren aan de ontdekking van nieuwe antibiotica. 
Acknowledgments 
Finishing a $\mathrm{PhD}$ thesis is a four year long journey that involves interactions between a vast numbers of people. The writing and finishing of this dissertation has been an amazing and interesting time and many people contributed with their help, discussions, questions, comments or suggestions. With these pages I want to thank all people who were involved in this work by supporting me in any way.

The first persons I want to gratitude and to say thank you very much are my two supervisors, Wietse and Paolina. I want to thank the both of you for your continuous and excellent support throughout the whole work contributing to this dissertation. I learned a lot from the both of you professionally and personally. Paolina, I couldn't had a better daily supervisor, I could always come to your office and talk and discuss with you anything that was on my mind. You also did a great job with keeping my motivation high if an experiment didn't worked out and had to be repeated.

Also many thanks to you Wietse, for keeping the overview and the "eagle eyes" on my projects, experiments and providing me with very helpful advices in our regular meetings, your (positive) critical comments and questions to my work guided my research to the right direction.

Thank you Jos for being my advisor during this time and for all your useful and constructive comments on manuscripts, especially on chapter two and on any other experimental plans.

Also a big thanks to you Hans van Veen, thanks for managing the project and for all your useful questions and advices during the BE-Basic project time.

Many thanks are also going to the people from the reception. Thank you Elly, Gerrie, Ninke, Edith and Patty, for your support and help. Thanks are also going to Dick, Eke and the facility team and to the department secretaries (Dep_Sec) Gerda, Maira and Els, thank you very much for helping me out on every occasion.

A big thank you to all of my current and former office-mates Thiago, Irene, Chunxu, Ruth S., Sarah J., Julia H. and Alex thanks for all the nice conversations and fun we had together during breaks and outside the office. 
Furthermore many thanks to all my colleagues and PhD fellows at the MEdepartment: Vivi, Yying, Ruth G., Kesia, Ohana, Juan, Lucas, Annelies, Desalegn, Noriko, Adrian, Lucas and Jason, thanks for all the nice talks and discussions and all the fun we shared! Big thanks also to Mauricio and Max for advices regarding the thesis print.

Jeff thanks for the nice conversations over guitars, metal music and nature!

I want to thank all the technicians at NIOO that were helping me during experiments. First of all I want to say many thanks to Saskia, thanks for your great help with the secondary metabolite extractions and the HPLCs. Especially big thanks for the help on extracting and identifying the Pederin like compound (remember 425 plates and 1,25 L of crude secondary metabolite extract). Marlies many thanks to you for the help during the transcriptome experiment. Thanks also to Maria, Roos, Roel and Marion for useful discussions and your help. Big thanks to Hans Z., thank you for measuring and handling all the traps and the data generated on our new GC/MS-Q-TOF. Also big thanks to Kees H., thank you for teaching me a lot about volatile organic compounds, GC/MS measurements and the use of linear retention indices. Furthermore I want to thank all the technicians from the molecular lab, Agaat, Tanja B., Crista and Freddy for helping me out on any occasion and supporting me with tips and advices in molecular work. Thanks to the ICT -team for helping me with setting up the two metabolomic servers, thanks to Hans R, Roy V, Stephan V. and Gerben for all technical support.

Thanks for fruitful conversations and the good team-work to my BE-Basic colleagues Tanja S. and Anna K.!

Thierry, many thanks to you for all the bioreporter assays you performed for me and the collaboration during the Paenibacillus/Burkholderia project.

Thanks to you Marco for the good collaboration during the Paenibacillus/Burkholderia project and your help in chemical characterization.

Victor C., thank you for all the useful chats about cloning, Burkholderia, antibiotics and all other things we discussed in the lab or during coffee break. 
Thanks to all of my colleagues for all the fun and everything else that we shared together, thank you: Julie C., Stijn, Veronica, Nico H., Stefan G., Gera H., Sang Yoon, Paolo, Maaike d.J., Viola K., Maaike v. A., Bart G., Sven, Jan K., and all other colleagues!

Nowadays in science a great bioinformatics knowledge is needed to handle all the "big data" therefore a big thanks to Victor de Jager, Hartelijke dank voor all je hulp met de bioinformatics. Thanks also to Mattias for the support with the sequence data during the Mucor project.

Many thanks go also to Judith Bauer and Prof. Harald Gross, thank you for your hospitality during my lab visit at the University Tübingen and your help and time with the Pederin like compound identification.

I also want to thank all students who helped me during some parts of my experiments. Most of them did their internships or their thesis together with me. Thank you, Lisa, for your help during the high-through-put screening (Chapter 2). Thank you, Cristina, for the help with the microcosm experiments (chapter 5). Thank you, Sytske, for starting a project about the silencing of antimicrobial activity together with Paolina and me during your Master Thesis.

Danke Alex für die nette Zusammenarbeit beim microcosm Experiment mit Pseudomonas, Pedobacter and Bacillus (Chapter 5).

Kristin, vielen Dank für die nette Zusammenarbeit während des Mucor Proects und all die konstruktiven Diskussionen über Volatiles, und qPCRs.

Thanks also to all friends for their support and fun during this time: Joop, Floris, Dennis, Axel, Robert, Daniel!

Simon, ich danke dir für deine Kameradschaft im ersten Jahr meines $\mathrm{PhD}$ projekts in Wageningen. Max, vielen dank für deine Kollegialität und die vielen tollen Gespräche über Wissenschaft, PC-games, R! und alles andere.

Vielen Dank an meine Eltern Werner and Hildegard. Ihr habt mich immer unterstützt und wart für mich da, ohne euch und eure unterstützung wäre es nicht das selbe gewesen. Auch vielen dank an Oma Elisabeth und Tante Martha!

Hartelijke dank aan jou Eveline. Dank je wel voor al je steun en hulp! 


\section{PE\&RC Training and Education Statement}

With the training and education activities listed below the PhD candidate has complied with the requirements set by the C.T. de Wit Graduate School for Production Ecology and Resource Conservation (PE\&RC) which comprises of a minimum total of 32 ECTS (= 22 weeks of activities)

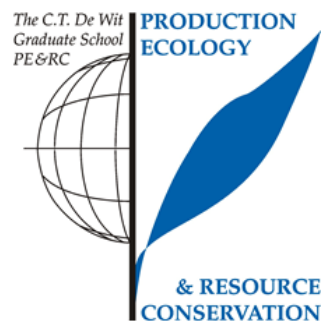

\section{Review of literature (4.5 ECTS)}

- Competition mediated discovery of cryptic encoding for novel antimicrobials

\section{Writing of project proposal (4.5 ECTS)}

- Competition mediated discovery of cryptic encoding for novel antimicrobials

\section{Post-graduate courses (5 ECTS)}

- Introduction to R for statistical analysis; PE\&RC (2011)

- Bioinformatics: an users approach; EPS (2013)

- Spring School host microbe interactions; WGS (2014)

- Microbial ecology; PE\&RC (2015)

\section{Laboratory training and working visits (3.9 ECTS)}

- Use of mass spectrometric techniques for identification of secondary metabolites produced by bacteria by using LESA and mass spectrometry techniques; Chemische Ökologie , Max-Plank-Institute Jena (2013)

- Identification of a Pederin like compound using VLC technique, NMR and HPLC techniques; Chemische Pharmakologie , Universität Tübingen (2015, 2016)

\section{Invited review of (unpublished) journal manuscript} ( 2 ECTS)

- Methods in Ecology and Evolution: Identification of response classes from heavy metal-tolerant soil microorganism communities by highly resolved concentration dependent screening in a microfluidic system (2014)

- Frontiers in Microbiology: Trichoderma volatiles effecting Arabidopsis: from inhibition to protection against pathogenic fungi (2015) 


\section{Competence strengthening / skills courses (2.7 ECTS)}

- Reviewing a scientific paper; WGS (2013)

- Scientific integrity; WGS (2013)

- Scientific publishing; WGS (2013)

- Information literacy PhD including Endnote introduction; WUR Library (2013)

- Scientific writing; Wageningen Into Languages (2014)

\section{PE\&RC Annual meetings, seminars and the PE\&RC weekend}

\section{(1.2 ECTS)}

- PE\&RC Day (2014)

- PE\&RC Day (2015)

- Current themes in ecology (2015)

- NERN Days (2016)

\section{Discussion groups / local seminars / other scientific} meetings (6.5 ECTS)

- NIOO PhD Literature discussion group meeting (2012-2013)

- Microbial Ecology group meeting (2012-2016)

- NIOO Microbial Ecology PhD group meeting (2014-2016)

\section{International symposia, workshops and conferences}

\section{(16.4 ECTS)}

- MiCom International student conference; Jena, Germany (2012)

- ICAR International conference on antibiotic research; Lissabon, Portugal (2012)

- FEMS; Leipzig, Germany (2013)

- BAGECO; Ljubjana, Slovenia (2013)

- SGM Society for general microbiology conference; Liverpool, UK (2014)

- SGM Society for general microbiology conference; Birmingham, UK (2015)

- Workshop volatile analysis; NIOO (2015)

- Micrope international symposium; Vienna, Austria (2015)

- KNVM Annual microbial ecology meeting; Amsterdam, the Netherlands (2016)

Lecturing / supervision of practicals / tutorials (3 ECTS)

- Practical student Course EABI; WUR (2014)

\section{Supervision of MSc student (3 ECTS)}

- Bacterial interactions and antibiotics (Sytske Drost) 


\section{Publications}

\section{Peer-reviewed and published articles:}

Garbeva, P., Tyc, O., Remus-Emsermann, M.N.P., Van Der Wal, A., Vos, M., Silby, M., and De Boer, W. (2011). No Apparent Costs for Facultative Antibiotic Production by the Soil Bacterium Pseudomonas fluorescens Pfo-1. Plos One 6. doi: ARTN e27266 doi: 10.1371/journal.pone.0027266.

Tyc, O., Van Den Berg, M., Gerards, S., Van Veen, J.A., Raaijmakers, J.M., De Boer, W., and Garbeva, P. (2014). Impact of interspecific interactions on antimicrobial activity among soil bacteria. Frontiers in Microbiology 5. doi: 10.3389/fmicb.2014.00567.

Tyc, O., Wolf, A.B., and Garbeva, P. (2015). The Effect of Phylogenetically Different Bacteria on the Fitness of Pseudomonas fluorescens in Sand Microcosms. Plos One 1o. doi: ARTN eo119838 10.1371/journal.pone.0119838.

Tyc, O., Zweers, H., De Boer, W., and Garbeva, P. (2015). Volatiles in interspecific bacterial interactions. Frontiers in Microbiology 6. doi: $10.3389 /$ fmicb.2015.01412.

Adam, E., Groenenboom, A., Kurm, V., Rajewska, M., Schmidt, R., Tyc, O., Weidner, S., Berg, G., De Boer, W., and Falcão Salles, J. (2016). Controlling the microbiome: microhabitat adjustments for successful biocontrol strategies in soil and human gut. Frontiers in Microbiology 7. doi: 10.3389/fmicb.2016.01079.

Schulz-Bohm, K., Tyc, O., De Boer, W., Peereboom, N., Debets, F., Zaagman, N., Janssens, T.K.S., and Garbeva, P. (2016) Fungus-associated bacteriome in charge of their host behavior. Fungal Genetics and Biology. doi: http://dx.doi.org/10.1016/j.fgb.2016.07.011. 


\section{Submitted articles:}

Olaf Tyc, Victor de Jager, Marlies van den Berg, Saskia Gerards, Thierry Janssens, Niels Zaagman, Marco Kai, Ales Svatos, Hans Zweers, Cornelis Hordijk, Harrie Besselink, Wietse de Boer and Paolina Garbeva

"Exploring bacterial interspecific interactions for discovery of novel antimicrobial compounds"

Stijn van Gils, Giovanni Tamburini, Lorenzo Marini, Arjen Biere, Maaike van Agtmaal, Olaf Tyc, Martine Kos, Roxina Soler, David Kleijn and Wim H. van der Putten

"Soil pathogen-aphid interactions under differences in soil organic matter and mineral fertilizer"

Olaf Tyc, Laura Tomás-Menor, Paolina Garbeva, Enrique Barrajón-Catalán and Vicente Micol

"Validation of the AlamarBlue ${ }^{\circledast}$ assay as a fast screening method to determine the antimicrobial activity of botanical extracts"

\section{Articles in preparation:}

Olaf Tyc, Chunxu Song, Michiel Vos and Paolina Garbeva

Review article

"The ecological role of secondary metabolites produced by soil bacteria" 
Olaf Tyc was born on the 25th of June 1981 in Balingen, Germany. After he attended a technical college for commerce and business administration and finishing his education as business administrator assistant in 2001 he did his civil service as paramedic at the German Red-Cross KV Zollernalb e.V.. In 2002 Olaf attended the Kolping-Kolleg in Stuttgart, Germany were he finished his high

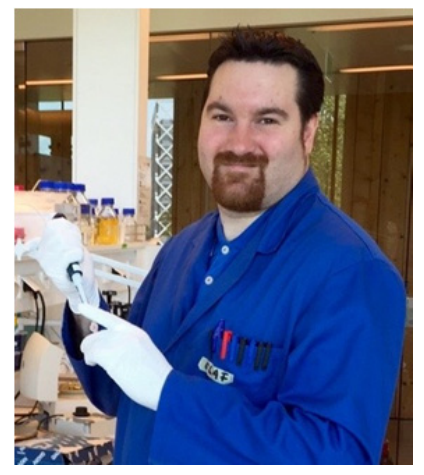
school degree in 2005. Driven by his great interest and curiosity for nature and microorganisms he decided to start a study related to biology, he therefore started to study agricultural biology at the Hohenheim University in Stuttgart, Germany. During his study his interests in microbiology was growing and particularly in microbemicrobe interactions. This interest brought him in 2009 to the Netherlands Institute of Ecology in Heteren were he did an ERASMUS internship and his diploma thesis in 2011, supervised by Prof. Dr. Andreas Kuhn (Hohenheim University, Stuttgart, Germany), Prof. Dr. Johannes A. van Veen and Dr. Paolina Garbeva (NIOO-KNAW, Netherlands). In his diploma thesis he studied the effect of interspecific bacterial interactions on the fitness and secondary metabolite production in Pseudomonas fluorescens Pfo-1. In 2012 Olaf started his $\mathrm{PhD}$ project "Competition-mediated discovery of novel antimicrobials" at the Netherlands Institute of Ecology (NIOO-KNAW) in Wageningen, which is presented in this thesis. At present he is a postdoctoral researcher at the department of Microbial Ecology at NIOO-KNAW. 
The research presented in this thesis was conducted at the Department of Microbial Ecology at the Netherlands Institute of Ecology (NIOO-KNAW) in Wageningen.

The financial support from the BE-Basic Foundation (www.be-basic.org) and the Netherlands Organization for Scientific Research (NWO) is gratefully acknowledged.

This is NIOO-Thesis number 132.

Cover design: Olaf Tyc

Printed by: Uitgeverij BOXpress: www.proefschriftmaken.nl 